Stephan Ruß

\title{
Telekommunikation als Standortfaktor für Klein- und Mittelbetriebe
}

Telekommunikative Entwicklungstendenzen und regionale Wirtschaftspolitik am Beispiel Hessen 
Stephan Ruß

\section{Telekommunikation als Standortfaktor für Klein- und Mittelbetriebe}

Die neuen Informations- und Kommunikationstechniken entwickeln sich zunehmend zu einem 'Zukunftsmarkt Telekommunikation', der zwangsläufig wirtschaftliche, räumliche, gesellschaftliche und soziale Umstrukturierungen zur Folge haben wird. Vor diesem Hintergrund werden Fragen nach der Bedeutung telekommunikativer Infrastruktur als Faktoren analysiert, die eine unternehmerische Standortwahl entscheiden. Die Analyse und Interpretation der durchgeführten primärempirischen Erhebung bildet die Grundlage für die Zusammenführung und Bewertung der empirischen und politischen Befunde. Abschluß der Arbeit stellt ein umfassender regionalpolitischer Handlungskatalog dar.

Stephan Ruß, geboren 1959 in Frankfurt, studierte von 1980 bis 1987 Wirtschaftsingenieurwesen an der Technischen Hochschule Darmstadt; Diplom 1987. Von 1987 bis 1990 absolvierte er ein Promotionsstudium an der Technischen Hochschule Darmstadt in Volkswirtschaftslehre, Fachgebiet Finanzwissenschaft; 1990 Promotion. 
Telekommunikation als Standortfaktor für Klein- und Mittelbetriebe 


\section{Sozialökonomische Schriften}

Herausgegeben von Bert Rürup

Band 4

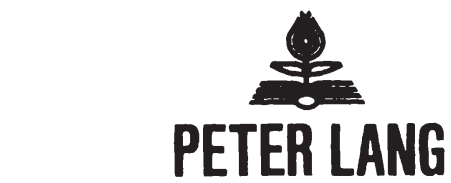

Franklurt am Main - Bern - Now York - Paris

Stephan Russ - 978-3-631-75107-7

Downloaded from PubFactory at 01/11/2019 08:15:47AM

via free access 


\section{Stephan Ruß}

\section{Telekommunikation als Standortfaktor für Klein- und Mittelbetriebe}

Telekommunikative

Entwicklungstendenzen und regionale Wirtschaftspolitik am Beispiel Hessen

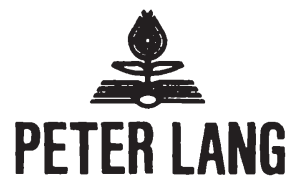

Frankfurt am Main - Bern - New York - Paris 


\title{
CIP-Titelaufnahme der Deutschen Bibliothek
}

Open Access: The online version of this publication is published on www.peterlang.com and www.econstor.eu under the international Creative Commons License CC-BY 4.0. Learn more on how you can use and share this work: http://creativecommons.org/licenses/ by/4.0.

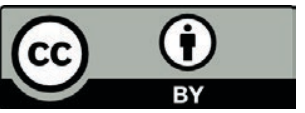

This book is available Open Access thanks to the kind support of ZBW - Leibniz-Informationszentrum Wirtschaft.

\section{RuB, Stephan:}

Telekommunikation als Standortfaktor fūr Klein- und Mittelbetriebe : telekommunikative Entwicklungstendenzen und regionale Wirtschaftspolitik am Beispiel Hessen / Stephan RuB. Frankfurt am Main ; Bem ; New York ; Paris : Lang, 1991

(Sozialőkonomische Schriften ; Bd. 4)

Zugl.: Darmstadt, Techn. Hochsch., Diss., 1990

ISBN 3-631-43762-5

NE: GT

\author{
D 17 \\ ISSN 0172-1747 \\ ISBN 3-631-43762-5 \\ ISBN 978-3-631-75107-7 (eBook) \\ (c) Verlag Peter Lang GmbH, Frankfurt am Main 1991 \\ Alle Rechte vorbehalten.
}

Das Werk einschließlich aller seiner Teile ist urheberrechtlich geschützt. Jede Verwertung außerhalb der engen Grenzen des Urheberrechtsgesetzes ist ohne Zustimmung des Verlages unzulāssig und strafbar. Das gilt insbesondere fūr Vervielfältigungen, Übersetzungen, Mikroverfilmungen und die Einspeicherung und Verarbeitung in elektronischen Systemen. 


\section{G E L E I T W O R T}

Die Kommunikations- und Informationstechnologien waren die gesellschaftlichen Schlüsseltechnologien des vergangenen Jahrzehnts und werden dies ausweislich aller valider technologischer Voraussagen auch in der nächsten Dekade sein. Da in den nächsten Jahren die entsprechende Infrastruktur - zumindest in den "Altländern" der Bundesrepublik aufgebaut sein wird, wird es möglich sein - die überaus beachtliche Rationalisierungspotentiale dieser Technologien - infolge ihrer Ketten- und Breitenwirkung in noch stärkerem Maße als bisher realisieren zu können.

Vor diesem Hintergrund stellt Herr Ruß die wissenschaftlich ambitiöse wie politisch aktuelle Frage nach der Bedeutung telekommunikativer Infrastrukturen für die unternehmerische Standortwahl insbesondere kleiner und mittlerer Betriebe.

Im einzelnen sind es die folgenden Aspekte, die Herr Ruß thematisiert:

- Ist die Telekommunikation ein Standortfaktor für Klein- und Mittelbetriebe. Inwieweit ist der Einfluß der Telekommunikationsförderung als Standortfaktor von Relevanz?

- Was sind die primären Adoptionsdeterminanten?

- Welches sind die regionalpolitischen Konsequenzen und Optionen?.

Bemerkenswert und erfreulich ist, daß Herr Ruß seine Überlegungen auf eine eigene, alle Branchen erfassenden empirische Datenerhebung begründet. Auch und gerade aus diesem Grunde ist seinen Ergebnissen ein überdurchschnittlich hohes Maß an praktischer Relevanz zuzumessen.

Angesichts der Aktualität des Themas ist es der Arbeit und dem Verfasser zu wünschen, daß diese Analyse nicht nur von einem Wissenschaftler für Wissenschaftler geschrieben wurde sondern auch von politischen Entscheidungsträgern als der Lektüre Wert erachtet wird denn Design und Befunde der Arbeit verdienen eine breitere Aufmerksamkeit.

Prof. Dr. Bert Rürup 
Stephan Russ - 978-3-631-75107-7

Downloaded from PubFactory at 01/11/2019 08:15:47AM

via free access 


\section{Inhalt}

Seite:

Tabellenverzeichnis $\quad 15$

Abbildungsverzeichnis $\quad 17$

0. Einleitung 21

1. Entwicklungstendenzen und Effekte der neuen telekommunikativen Techniken

1.1. Definition und Abgrenzung der neuen Telekommunikationstechniken

1.1.1. Begriffsbestimmung 25

1.1.1.1. Terminologie 25

1.1.1.2. Abgrenzung der Individualkommunikation 28

1.1.2. Die neuen Telekommunikationstechniken 29

1.1.2.1. Überblick 29

1.1.2.2. Sprachkommunikation 31

1.1.2.3. Daten- und Textkommunikation 34

1.1.2.4. Bildkommunikation $\quad 39$

1.2. Entwicklungsstrukturen der Telematik 42

1.2.1. Erwarteter Telekommunikationsbedarf in
Hessen

1.2.1.1. Einführung 42

1.2.1.2. Empirische Befunde der Gesellschaft für Information und Dokumentation 43

1.2.1.3. Prognose der Deutschen Bundespost 49

1.2.2. Gegenwärtige Infrastruktur und Agglomeration der Telekommunikation 50

1.2.2.1. Infrastuktur der Bundesrepublik Deutschland 
1.2.2.2. Agglomerationen in Hessen

1.2.3. ISDN-Entwicklungskonzept der Deutschen Bundespost

1.2.3.1. ISDN-Grundlagen $\quad 56$

1.2.3.2. ISDN-Ausbaustrategie 58

1.2.3.3. ISDN-Tarife 60

1.3. Effekte der Telematik 62

1.3.1. Gesamtwirtschaftliche Effekte der Telematik

1.3.1.1. Generelle Aspekte 63

1.3.1.2. Beschäftigungseffekte 64

1.3.2. Regionalpolitische Effekte der Telematik 69

1.3.2.1. Gegenwärtige Entwicklungstendenzen 69

1.3.2.2. Wirkungsthesen der Telematik 71

1.3.2.3. Ambivalenz räumlicher wirkungen 77

1.3.3. Beschäftigungseffekte als Bestimmungsfaktor $\begin{array}{ll}\text { regionalpolitischer Wirkungen } & 78\end{array}$

1.3.3.1. Analogien zur Diffusion von Industrierobotern

1.3.3.2. Regionalpolitische Konsequenzen der Telematikdiffusion

2. Telekommunikationsförderung aus der Perspektive der regionalen Wirtschaftspolitik

2.1. Aspekte der regionalen Wirtschaftspolitik 86

2.1.1. Grundsätze und Koordinationsproblematik 86

2.1.1.1. Zum Begriff 86

2.1.1.2. Strategien und ziele 87

2.1.1.3. Rechtliche Grundlagen 96

2.1.1.4. Koordinationsproblematik 101

2.1.2. Trends in den Konzepten der regionalen wirtschaftspolitik 
2.1.2.1. Von der exogenen zur endogenen Beeinflussung

2.1.2.2. Von der Nachfrageorientierung hin zur Angebotsorientierung

2.1.2.3. Standortfaktorwechsel

2.1.3. Regionalpolitischer Handlungsbedarf

2.2. Förderungsmöglichkeiten telekommunikativer Techniken

2.2.1. Förderung der Einführung und Anwendung

2.2.1.1. Direkte Subventionierung

2.2.1.1.1. Investitionshilfen

2.2.1.1.2. Subventionierung von individuellen Beratungen

126

2.2.1.2. Informations- und Beratungskonzepte

2.2.1.2.1. Information und Beratung durch die Deutsche Bundespost

2.2.1.2.2. Innovations- und Technologieberatungsstellen

$2 \cdot 2 \cdot 1 \cdot 3$. Lokale und regionale Telematikvorhaben

2.2.1.3.1. Teleport und Telehaus

$2 \cdot 2 \cdot 1 \cdot 3 \cdot 2$. Technologie- und Gründerzentren

2.2.1.3.3. Einschlägige Informations- und Beratungseinrichtungen

2.2.1.3.4. Öffentliche Betriebsversuche

2.2.2. Infrastrukturpolitik

2.2.2.1. Gebührenpolitik unter regionalpolitischem Aspekt

$2 \cdot 2 \cdot 2 \cdot 2$. Entwicklungstendenzen und raumstrukturelle Relevanz der Telekommunikationsgebühren

2.2.2.3. Regionale Infrastrukturpolitik

2.3. Beurteilung der regionalen Wirtschaftspolitik unter dem Aspekt veränderter Rahmenbedingungen 
2.3.1. Veränderung der Rahmenbedingungen

2.3.1.1. Allgemeine Aspekte

2.4.1.2. Veränderung des betrieblichen standortverhaltens

2.3.2. Konsequenzen für die regionale wirtschaftspolitik

2.3.2.1. Bedeutungsgewinn der Bauleit- und Regionalplanung

2.3.2.2. Zur Neuorientierung raumordnungspolitischer Handlungskonzepte

3. Einzelbetriebliche Untersuchung des Einflusses der Telematik auf die standortentscheidung von Kleinund Mittelbetrieben

3.1. Kennzeichnung der Untersuchung

3.1.1. Grundlagen und ziele

3.1.1.1. Untersuchungsansatz

3.1.1.2. Arbeitshypothese und abgeleitete Unterhypothesen

3.1.1.3. Zielsetzung der Erhebung

3.1.2. Konzept und Realisierung der Erhebung

3.1.2.1. Auswahl und Abgrenzung der Unternehmen und Untersuchungsgebiete

3.1 .2 .2 . Erhebungsmethodik

3.1.2.3. Stichprobenumfang, Rücklauf und Repräsen$\operatorname{tanz}$

3.1.3. Statistische Auswertungsverfahren

3.2.1. Struktur der antwortenden Unternehmen

3.2.1.1. Regionale Verteilung und Branchenstruktur

3.2.1.2. Beschäftigtenzahl und Unternehmensumsatz 
3.2.1.3. EDV-Ausstattung und Forschungs- und Entwicklungsaktivitäten

3.2.1.4. Art und Gründungsjahr des Unternehmens

3.2.2. Einstellung der Unternehmen gegenüber der Telematik

3.2.2.1. Kenntnis- und Adoptionsstand

$3 \cdot 2 \cdot 2 \cdot 2$. Bedeutung der Dienste für den eigenen Betrieb

3.2.2.3. Adoptionshemmisse

3.2.2.4. Endgerätepreiseinfluß auf die Adoption

3.2.3. Standorteinschätzung und Standortverhalten

3.2.3.1. Einschätzung des Standortes

3.2.3.2. Einfluß der Standortfaktoren auf die Standortwahl

3.2.3.3. Informationsquellen zur standortwahl

3.2.4. Telekommunikationsfördermaßnahmen

3.2.5. Kommunikationsverhalten

3.2.5.1. Kommunikationsintensität

3.2.5.2. Interne und externe Kommunikationsarten

3.2.5.3. Substitutionspotential persönlicher Kontakte

3.3. Interpretation und Zusammenfassung der Ergebnisse der empirischen Untersuchung

3.3.1. Telekommunikation als Standortfaktor

3.3.1.1. Einfluß der Telekommunikation auf die Standortwahl

3.3.1.2. Standortaufwertung durch Förderung neuer Telekommunikationstechniken

3.3.2. Adoption neuer telekommunikativer Techniken 
3.3.2.2. Einflußgrößen der Adoption

3.3.2.3. Adoptionshemmnisse

4. Beurteilung der Telekommunikationsförderung als regionalpolitisches steuerungsinstrument

4.1. Grundsätzliche Überlegungen zur Akzeptanz neuer Techniken

4.1.1. Kritik an den Netzausbauplänen der Deutschen Bundespost

4.1.2. Regionale und soziostrukturelle Disparitäten der Akzeptanz neuer Techniken

4.2. Beurteilung der Telekommunikationsfördermaßnahmen

4.2.1. Beurteilung der Telekommunikation als Standortfaktor

4.2.2. Beurteilung der finanziellen Anreize

4.2.3. Beurteilung der Realtransfers

4.2.4. Beurteilung der Anregung und Förderung von Kooperationen

4.2.5. Beurteilung infrastrukturpolitischer Maßnahmen

4.3. Telekommunikationsförderung im Ausland

4.3.1. Informationstechnologische Lokalzentren als Instrument der Regionalpolitik in Norwegen

4.3.2. Einführung des Télétel-Systems in Frankreich

4.4. Notwendige Maßnahmen zur Förderung der neuen telekommunikativen Techniken

4.4.1. Allgemeine Maßnahmen zur Akzeptanzsteigerung

4.4.2. Anwendungsförderung durch ISDN-SystemMarketing

4.4.3. Förderung bis zur Mindestteilnehmerzahl 
5. Befunde und politische optionen

290

Literaturverzeichnis 298

Anhang

319 
Stephan Russ - 978-3-631-75107-7

Downloaded from PubFactory at 01/11/2019 08:15:47AM

via free access 
Tabelle 1.2: Nutzung von und Interesse an Telekommunikationsdiensten nach Sektoren

Tabelle 1.3: Grundgebühr für den ISDN-Anschluß

Tabelle 2.1: Vergleich der Fernmeldegebührenanteile ausgewählter Wirtschaftsbereiche in $\%$ der Gesamtkosten im Durchschnitt der Jahre 1978-1980

Tabelle 2.2: Entfernungsabhängigkeit von Telekommunikationsdiensten

Tabelle 2.3: Unterschiede zwischen mobilitätsorientierter Förderstrategie und innovationsorientierter Regionalpolitik

Tabelle 3.1: Einzel- und Gesamtindikatoren der hessischen Arbeitsmarktregionen

Tabelle 3.2: Verwendete statistische Verfahren

Tabelle 3.3: Kennzeichung des Signifikanzniveaus

Tabelle 3.4: Interpretation des Korrelationskoeffizienten

Tabelle 3.5: Art der Unternehmen

Tabelle 3.6: Adoptionsverhalten gegenüber den Telekommunikationsdiensten 
Tabelle 4.1: Einschätzung der neuen Techniken als "Chance" nach Regionstyp und Erwerbstätigkeit in $\%$

Tabelle 4.2: Einschätzung der neuen Techniken als "Chance" nach Erwerbstätigkeit im Nord-Süd-Vergleich in $\%$

Tabelle 4.3: Akzeptanz der neuen Techniken nach sozialstrukturellen Merkmalen in \%

Tabelle 4.4: Phasen des ISDN-Innovationsmarketing und verfolgte $\mathrm{ziele}$ 
Abbildung 1.2: Übersicht über die neuen Telekommunikationstechniken

Abbildung 1.3: Schätzung des zukünftigen Übertragungsvolumens

Abbildung 1.4: Vorläufer-Breitband-Netz

Abbildung 1.5: Ausbauplanung der Deutschen Bundespost

Abbildung 1.6: Beschäftigungswirkungen der Diffusion von Industrierobotern

Abbildung 2.1: Intendiertes zielsystem der praktizierten regionalen Wirtschaftspolitik

Abbildung 2.2: Abgrenzung der Standortfaktoren

Abbildung 2.3: Technologie- und Gründerzentren

Abbildung 3.1: Fördergebiet der Gemeinschaftsaugabe "Verbesserung der regionalen Wirtschaftsstruktur" des 17. Rahmenplans

Abbildung 3.2: Prozentuale Verteilung der Antworten auf die einzelnen Regionen

Abbildung 3.3: Prozentuale Verteilung der Antworten auf die einzelnen Branchen

Abbildung 3.4: Antwortverhalten nach Region und Branche 
Abbildung 3.5: Gewichtung der Sektoren nach Regionen 201

Abbildung 3.6: Anzahl der Unternehmen nach Beschäftigengrößenklassen

Abbildung 3.7: Anzahl der Unternehmen nach Umsatzklassen in $\%$

Abbildung 3.8: PC-Ausstattung der Unternehmen 204

Abbildung 3.9: Forschung und Entwicklung in den Unternehmen

Abbildung 3.10: Anzahl der Unternehmen mit mehreren Standorten

Abbildung 3.11: Gründungsjahr der Unternehmen

Abbildung 3.12: Gründungsjahr der Unternehmen in Klassen

Abbildung 3.13: Mittelwerte des Adoptionsverhaltens

Abbildung 3.14: Mittelwerte des Adoptionsverhaltens nach Regionen

Abbildung 3.15: Bedeutung der Telekommunikationsdienste

Abbildung 3.16: Bedeutung der Dienste nach Regionen

Abbildung 3.17: Bedeutung der Dienste nach Branchen

Abbildung 3.19: Regionale Gewichtung der Adoptionshemmisse 
Abbildung 3.20: Mittélwerte der potentiellen Endgeräteanschaffungspreise

Abbildung 3.21: Mittelwerte der potentiellen Endgeräteanschaffungspreise nach Regionen

Abbildung 3.22: Momentane Standortbewertung

Abbildung 3.23: Momentane standortbewertung des ISDN-Anbindungszeitpunktes

Abbildung 3.24: Standorteinschätzung ausgewählter Faktoren nach Regionen

Abbildung 3.25: Einfluß der Standortfaktoren auf die standortwahl

Abbildung 3.26: Einfluß des ISDN-Anbindungszeitpunktes auf die standortwahl

Abbildung 3.27: Einfluß der Telekommunikations-Verfügbarkeit auf die standortwahl

Abbildung 3.28: Einfluß der Telekommunikations-Standortfaktoren nach Regionen

Abbildung 3.29: Einfluß der TelekommunikationsStandortfaktoren nach Branchen

Abbildung 3.30: Informationsquellen zur Standortwahl 236

Abbildung 3.31: Einfluß der Fördermaßnahmen auf die standorteinschätzung

Abbildung 3.32: Einfluß der Fördermaßnahmen auf die Standorteinschätzung nach Regionen 
Abbildung 3.33: Einfluß der Fördermaßnahmen auf die Standorteinschätzung nach Branchen

Abbildung 3.34: Einfluß der Fördermaßnahmen auf die standorteinschätzung nach Gründungszeitpunkt

Abbildung 3.35: Fernmeldegebühren in DM pro Monat

Abbildung 3.36: Endgerätekosten in DM pro Monat

Abbildung 3.37: Kommunikationsarten intern

Abbildung 3.38: Kommunikationsarten extern

Abbildung 3.39: Substitutionspotential persönlicher Kontakte durch neue Telekommunikationstechniken

Abbildung 3.40: Können persönliche Kontakte durch neue Telekommunikationstechniken ersetzt werden? 


\section{Einleitung}

Die neuen Informations- und Kommunikationstechniken entwikkeln sich zunehmend $z u$ einem 'Zukunftsmarkt Telekommunikation', der zwangsläufig wirtschaftliche, räumliche, gesellschaftliche und soziale Umstrukturierungen zur Folge haben wird. Ein Großteil der neuen Telekommunikationsdienste und -geräte steht bereits heute zur Disposition beziehungsweise kurz vor der Einführung, ohne daß ein Konsens bezüglich der Effekte der neuen Telekommunikationstechniken existiert.

Größere Umwälzungen werden im Büro- bzw. Verwaltungsbereich erwartet und Schlagworte wie Büro-Taylorismus, Job-Killer Mikroelektronik und Bürorationalisierung kursieren seit längerem in den Medien. Trotz der primären Ausrichtung der Informationstechnologien auf die Rationalisierung der Büros, ist die Wirkung nicht auf die Betriebe beschränkt. Die 'Automatisierung der Kopfarbeit' wird lediglich als ein Veränderungsimpuls angesehen, wogegen die neuen Telekommunikationstechniken alle Lebensbereiche, auch die privaten, erreichen und das gesellschaftliche Leben insgesamt verändern werden.

Die 'euphorischen' Erwartungen während der ersten Hälfte der 80er Jahre, im besonderen in Bezug auf das Dezentralisierungspotential der neuen Telekommunikationstechniken, haben sich nicht bestätigt. Gegenwärtig wird aus großräumlicher sicht eine zunehmende Konzentration der strukturstarken Agglomerationen und aus kleinräumlicher sicht eine Dezentralisierung und somit eine Ausdehnung der Einzugsbereiche der städte prognostiziert, was resultierend zu einer Verschärfung der regionalen Disparitäten führt.

Aufgrund der bisherigen Diffusion lassen sich 'Adoptionsdeterminanten' ableiten, die potentielle Ansatzpunkte einer regionalpolitischen steuerung darstellen. Die primären re- 
gionalpolitischen Aktionsfelder konzentrieren sich auf Maßnahmen im Bereich:

- der Wirtschaftsförderung,

- der wissenschaftlich-technischen Infrastruktur,

- der Arbeitsmarkt- und Qualifikationspolitik und

- der Infrastrukturpolitik.

Ausgehend von den Entwicklungsperspektiven der Telekommunikation wird ein regionalpolitischer Handlungsbedarf evident, der eine Analyse der Möglichkeiten einer Instrumentalisierung im Dienste der regionalen Wirtschaftspolitik bedingt.

Was bisher jedoch weitgehend fehlt, sind gesicherte und vergleichbare Informationen über das standort- und Adoptionsverhalten der Unternehmen. Entsprechende Forschungsdefizite sind zweifelsohne partiell das Resultat der noch vergleichsweise jungen Geschichte der neuen telekommunikativen Techniken in der Bundesrepublik Deutschland.

Ein Forschungsdefizit allein begründet selbstverständlich noch keinen Forschungsbedarf. Letzterer leitet sich vielmehr $\mathrm{ab}$ aus der in den letzten Jahren zu beobachteten Diffusion der neuen Telekommunikationstechniken und den damit verbunden Chancen einer Belebung der regionalen wirtschaftsentwicklung, als auch aus der inhärenten Gefahr von Fehlallokationen, was einer Verschwendung öffentlicher Mittel, bedingt durch die bereits existierenden Telekommunikationsfördermaßnahmen, gleichkäme.

Übergeordnetes ziel dieser Arbeit ist die Analyse der potentiellen chancen der neuen telekommunikativen Techniken als Instrument der regionalen Wirtschaftspolitik. Dazu ist es notwendig das spezielle Datendefizit hinsichtlich des standort- und Adoptionsverhaltens zu beheben. 
Aus dem Spannungsfeld zwischen der Diffusion neuer telekommunikativer Techniken und den prognostizierten regionalpolitischen Effekten, ergeben sich für diese Arbeit folgende zentrale Fragestellungen:

- Ist Telekommunikation ein standortfaktor für klein- und Mittelbetriebe?

- Inwieweit ist der Einfluß der Telekommunikationsförderung als Standortfaktor von Relevanz?

- Was sind die primären Adoptionsdeterminanten?

- Welche regionalpolitischen Konsequenzen resultieren aus den Ergebnissen der Untersuchug?

Um das o.a. Hauptziel zu erreichen wird wie folgt vorgegangen. Im ersten Kapitel, das Grundlagencharakter hat, wird nach einer Klärung der wichtigsten begrifflichen Voraussetzungen und der, für das Verständnis der Fragestellungen im empirischen Teil notwendigen, Entwicklungsstrukturen der Telematik, die regionalpolitische Relevanz, anhand der qualitativen und quantitativen Einschätzungen der Effekte der Telematik, herausgearbeitet.

Im zweiten Kapitel erfolgt eine Analyse der wichtigsten regionalpolitischen Rahmenbedingungen, als grundlegende Parameter einer Telekommunikationsförderung. Auf dieser Grundlage aufbauend wird eine vertiefende Betrachtung hinsichtlich der praktischen Telekommunikationsfördermöglichkeiten in der Bundesrepublik Deutschland vorgenommen. Im AnschluB wird die regionale wirtschaftspolitik unter dem Aspekt der aktuellen, veränderterten Rahmenbedingungen beurteilt.

Für das weitere Vorgehen ist, aufgrund der mangelnden Datenbasis, eine primärempirische Erhebung durchgeführt worden (Kapitel 3). Die Erhebung wurde, exemplarisch für den Bereich Hessen, in Form einer schriftlichen Befragung ausgewählter Unternehmen vorgenommen. Neben den grundsätzlichen Fragestellungen bezüglich des Informations- und Adop- 
tionsstandes, wurde das standortverhalten und weitere, die Diffusion der Telekommunikationstechniken determinierende, Faktoren analysiert und anschließend interpretiert.

In Kapitel 4 werden zunächst grundsätzliche Überlegungen zur Akzeptanz neuer Techniken vorgestellt und anschließend, basierend auf den Befunden der empirischen Erhebung, eine Zusammenführung und Bewertung der Telekommunikationsfördermaßnahmen vorgenommen. Die zum Teil wesentlich längeren Erfahrungen aus dem Ausland werden beispielhaft anhand der Entwicklung in Norwegen und Frankreich dargelegt, um zu prüfen, ob und in welchen Punkten eine Übertragbarkeit der dortigen Erfolgsrezepte und Mißerfolgsgründe möglich und sinnvoll ist. Auf dieser Grundlage werden die für die Bundesrepublik Deutschland notwendigen Maßnahmen zur Förderung der neuen telekommunikativen Techniken herausgearbeitet.

Die abschließenden Befunde und politischen Optionen (Kapitel 5) umfassen eine thesenartige Zusammenstellung der erarbeiteten Ergebnisse und die abgeleiteten, auf den regionalpolitischen Konsequenzen basierenden, Handlungsempfehlungen. 
1. Entwicklungstendenzen und Effekte der neuen telekommu-
nikativen Techniken

\subsection{Definition und Abgrenzung der neuen Telekommunika- tionstechniken}

\subsubsection{Begriffsbestimmung}

\subsubsection{Terminologie}

Basierend auf den technischen Entwicklungen der vergangenen Jahre $^{1}$ sind verschiedene neue Informations- und Kommunikationstechniken entwickelt worden, die zum Teil bereits heute von der Deutschen Bundespost als Dienstleistung angeboten werden und für die Zukunft Perspektiven für völlig neuartige Dienstleistungen eröffnen. Wesentliche Bestandteile dieser Entwicklungen sind:

- die qualitative Verbesserung bestehender Dienste,

- die quantitative Erweiterung durch neue Dienste,

- die Integration bestehender und neuer Dienste durch Digitalisierung sowie

- die Ropplung von Informationsübertragung und -verarbeitung. ${ }^{2}$

Das diesen Entwicklungen implizite wirkungspotential wird in der Literatur durch eine Vielzahl von Publikationen diskutiert, in denen Begriffe wie 'Telekommunikation', 'neue

1 Besonders im Bereich der hier relevanten Digitaltechnik.

2 Vgl. Spehl, H.: Räumliche wirkungen der Telematik, in: Raumforschung und Raumordnung Nr. 6, 43. Jahrgang, 1985, S. 254 ; Wagner, P.: Gratwanderung oder Durststrecke Probleme mit ISDN, in: Frankfurter Allgemeine Zeitung $\mathrm{Nr}$. 241 vom 17. Oktober 1989, S. B26. 
Informations- und Kommunikationstechniken', 'Telematik' und 'Neue Medien' verwendet werden, ohne daß eine einheitliche Definition vorliegt. Für die folgenden Ausführungen wird deshalb eine Begriffsfestlegung vorgenommen.

In dieser Arbeit wird unter Telekommunikation insbesondere der Bereich der unternehmensexternen Kommunikation ${ }^{3}$ behandelt, der auf dem öffentlichen Kommunikationsnetz und den von der Deutschen Bundespost angebotenen Diensten basiert.

Mit den synonymen neue Informations- und Rommunikationstechniken, neue telekommunikative Techniken und neue Telekommunikationstechniken werden alle technischen Geräte und Einrichtungen bezeichnet, die Informationen ${ }^{4}$ aller Art verarbeiten, speichern oder übertragen können.

Der Begriff Neue Medien läßt sich nicht exakt definieren. Als Sammelbegriff umschließt er einerseits gänzlich neue Medientechniken, andererseits lediglich technisch modifizierte Verfahren. ${ }^{5}$ Verbunden wird dieser Begriff primär mit den politischen Auseinandersetzungen um Kabelfernsehen, Privates Fernsehen und Videokassetten. ${ }^{6}$ Aus diesen Gründen wird der Begriff Neue Medien in dieser Arbeit nicht verwendet.

Der Begriff Telematik ist eine Wortschöpfung der Franzosen Simon Nora und Alain Minc und wurde 1978 geprägt. 7 Er um-

3 In Abgrenzung zur Bürokommunikation.

4 Unter Information per se wird ein Signal beliebigen Inhalts verstanden.

5 Vgl. Ratzke, D.: Handbuch der Neuen Medien, Stuttgart 1982 , S. 14 .

6 Vgl. Kaiser, W.: Entwicklungslinien der Telekommunikation, in: Konrad Adenauer Stiftung (Hrsg.): Neue Medien, Melle 1984, S. 67-74.

7 Vgl. Nora, S.; Minc, A.: Die Informatisierung der Gesellschaft, Frankfurt, New York 1978. 
faßt die Integration von Telekommunikation und Informatik in Bezug auf die wachsende Verknüpfung von Informationsverarbeitung und -übertragung, die in Abbildung 1.1 dargestellt ist.

Abbildung 1.1: Telematik 8

GRUNDLAGEN: Kupferkabel, Glasfaserkabel, Satelliten, Mikrochips, Digitale vermittlung usw.

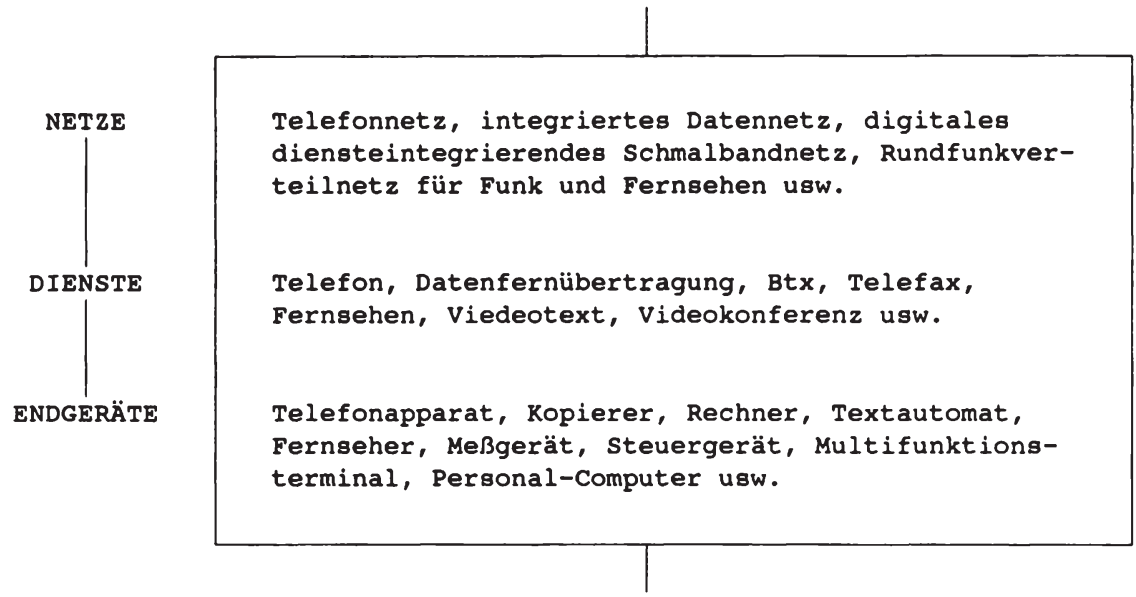

Anwendungen: Integration in: Betrieben, Verwaltungen und Haushalten

Aufgrund der dynamischen Entwicklung im Telematikbereich vermischen sich verstärkt die Begriffe Individual- und Massenkommunikation. Die in dieser Arbeit zu untersuchenden wirkungen konzentrieren sich auf die Individualkommunikation. Im folgenden soll deshalb versucht werden, eine Abgrenzung von Individual- und Massenkommunikation vorzunehmen.

8 In Anlehnung an Lange, S.: Telematik und regionale wirtschaftspolitik, Köln 1985, S. 11. 


\subsubsection{Abgrenzung der Individualkommunikation}

Der Unterschied zwischen der Individualkommunikation und der Massenkommunikation wird in den Diskussionen um die Wirkungen der Telematik häufig nicht hinreichend berücksichtigt. 9 Bei der Individualkommunikation wird in der Regel eine individuelle Verbindung von zwei oder mehreren Teilnehmern vermittelt. 10 Jede Netzendstelle kann mit jeder anderen Netzendstelle verbunden werden und einen Dialog führen. Im Gegensatz zur Individualkommunikation, werden bei der Massenkommunikation über Verteilnetze, von einer Zentralstelle aus, Informationen an mehrere Teilnehmer verteilt. Typische Verteilnetze sind beispielsweise das Rundfunksendernetz und die Breitbandverteilnetze. Verteilnetze sind in der Regel für eine einseitige Kommunikation ausgelegt.

Unter Berücksichtigung der ökonomischen und raumstrukturellen Wirkungen der Telematik, ist die Massenkommunikation von nachrangiger Bedeutung. Ausschlaggebend ist die Individualkommunikation, die mit Hilfe der bestehenden Netze, bzw. nach dem vollständigen Aufbau des Breitbandnetzes, die wirtschaftliche und räumliche Entwicklung stark beeinflussen kann. 11

Die Behandlung möglicher Effekte der Telematik wird im Rah-

9 Vgl. Hoberg, R.: Raumwirksamkeit neuer Kommunikationstechniken - Innovations- und diffusionsorientierte Untersuchung, in: Vorstand der Gesellschaft für Regionalforschung e.v. (Hrsg.): Jahrbuch für Regionalwissenschaft, Göttingen 1983, s. 8 .

10 Die Vermittlung geschieht meistens uber das klassische Vermittlungsnetz der Deutschen Bundespost, das Fernsprechnetz.

11 Vgl. Naylor; H., Türke, K.: Welche Wirkungen können neue Kommunikationsmedien auf Raumordnung und Stadtentwicklung haben?, in: Informationen zur Raumentwicklung, Heft 3 , 1982, S. 175 . 
men dieser Arbeit im wesentlichen auf Formen der Individualkommunikation beschränkt.

\subsubsection{Die neuen Telekommunikationstechniken}

\subsubsection{1. Überblick}

Durch das Zusammenwachsen einzelner Kommunikationselemente sind aus Sprach-, Bild-, Text- und Datenkommunikation eine Vielzahl von Telekommunikationsdiensten und unterschiedlichen Netzen entstanden, die in Abbildung 1.2 dargestellt sind. Die Darstellung der Telekommunikationsdienste beschränkt sich auf bereits von der Deutschen Bundespost eingeführte oder in Planung befindliche Dienste.

Unter neuen Telekommunikationstechniken werden zum einen Erweiterungen herkömmlicher Kommunikationstechniken und zum anderen völlig neu entwickelte Techniken und Dienste verstanden.12 Ein wesentliches Innovationsmerkmal der neuen Telekommunikationstechniken stellt die Integration verschiedener Dienste dar, wobei unter 'Integration' die Abwicklung verschiedener Telekommunikationsformen in einem einheitlichen Netz und die option zur simultanen Inanspruchnahme dieser Dienste verstanden wird. 13 Technische Voraussetzung der Integration ist die Einführung der Digitaltechnik, wobei die Umstellung von Analog- auf Digitaltechnik zusätzlich eine erhöhte Informationsübertragungska-

12 Vgl. Bundesministerium für das Post- und Fernmeldewesen: Konzept der Deutschen Bundespost zur Weiterentwicklung der Fernmeldeinfrastruktur, Bonn 1984, S. 4.

$13 \mathrm{Vgl}$. Bundesministerium für das Post- und Fermeldewesen (Hrsg.): Das ISDN-Angebot der Deutschen Bundespost - Ein örtlicher und zeitlicher Überblick, Bonn 1988, S 4 f. 
Abbildung 1.2: Übersicht über die neuen Telekommunikationsformen

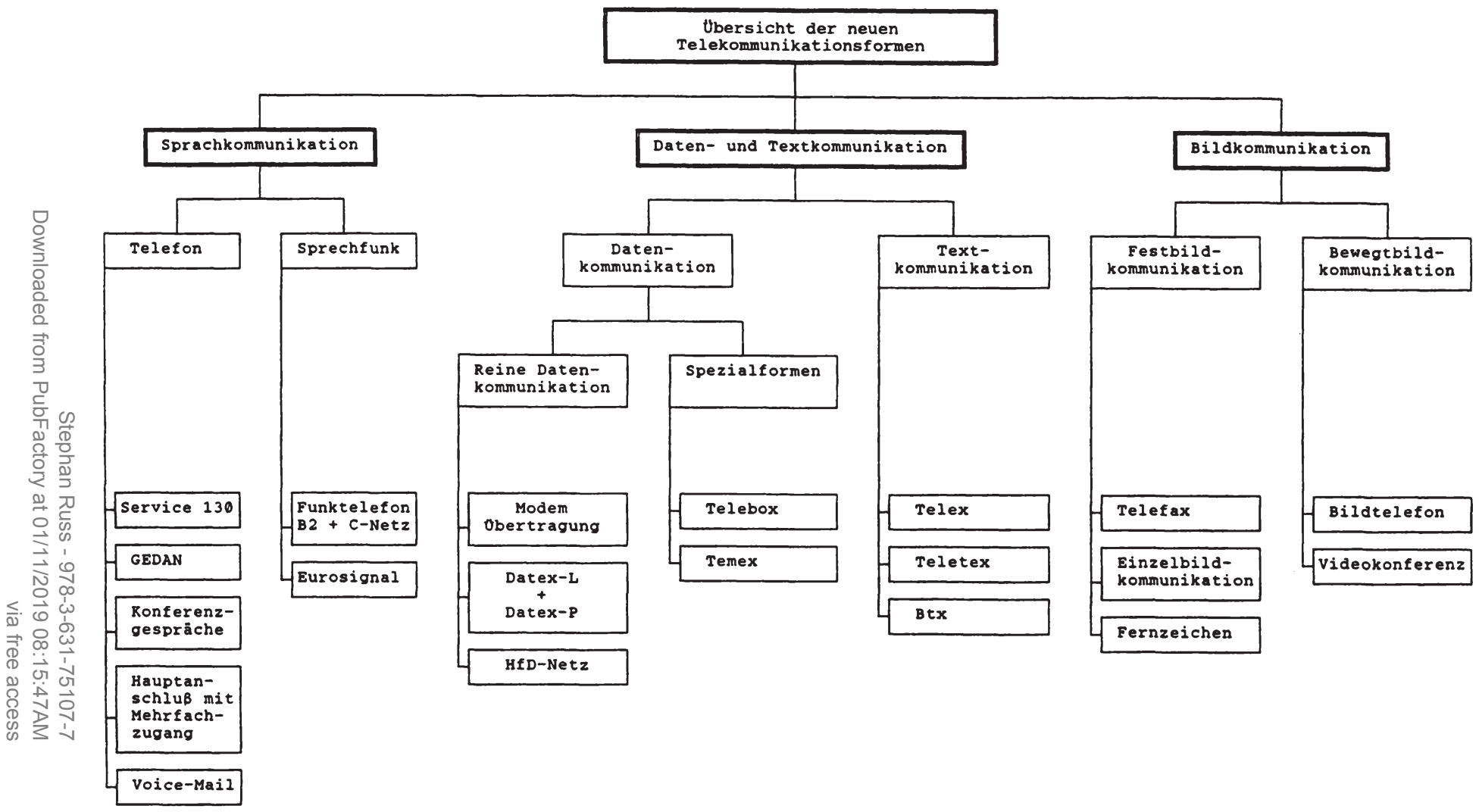


pazität existierender Leitungen, eine verbesserte speicherung von Informationen und eine vereinfachte Weiterverarbeitung der übertragenen Daten beim Empfänger ermöglicht. ${ }^{14}$

Prinzipiell erlauben die technischen Möglichkeiten im Bereich der neuen telekommunikativen Techniken eine Vielzahl von Nutzungsmöglichkeiten. Die im Rahmen dieser Arbeit dargestellten Telekommunikationstechniken konzentrieren sich auf die von der Deutschen Bundespost angebotenen Dienste, die bereits zum größten Teil flächendeckend über das vorhandene Fernsprechnetz angeboten werden. Die Vielzahl an neuen Telekommunikationstechniken, die von der Privatwirtschaft angeboten werden, sind nicht Bestandteil der Betrachtung, da sie momentan nur 'inhouse' oder über firmeneigene Netze realisierbar und primär für größere Unternehmen relevant sind.

\subsubsection{8prachkommunikation}

Die elektronische Sprachkommunikation und der Sprechfunk stellen die am weitesten verbreitete Telekommunikationsform der Gegenwart dar. In der Bundesrepublik Deutschland beispielsweise werden täglich über 40 Millionen orts-, $23 \mathrm{Mil-}$ lionen Fern- und eine Million internationale Gespräche durchgeschaltet.15 Entwicklungen in Richtung 'Mensch-Maschine-Dialog' lassen für die absehbare zukunft noch keine gravierenden neuen Veränderungen erwarten.

Im Mittelpunkt der fortschreitenden Entwicklungen im Bereich der Sprachkommunikation steht die Mikroelektronik,

14 Vgl. Ewers, H.; Fritsch, M.: Telematik und Raumentwicklung, a.a.0., s. 20 .

15 Vgl. Schinagl, W.: Neue Dienste der Deutschen Bundespost, Fernmeldetechnisches Zentralamt der Deutschen Bundespost (Hrsg.), Darmstadt 1987, S. 12. 
die eine Weiterentwicklung des Fernsprechens durch zusätzliche technische Einrichtungen wie rechnergesteuerte Vermittlungssstellen ermöglicht. Im folgenden werden die neuen Leistungsmerkmale des Telefondienstes und des sprechfunks erläutert.

\section{Telefondienste 16}

Der service 130 bietet die Möglichkeit, bundesweit ${ }^{17}$, mit Hilfe einer bundeseinheitlichen Vorwahlnummer '0130', zum ortstarif $^{18}$ erreichbar $z u$ sein, ohne daß flächendeckende Geschäftsstellen vorhanden sein müssen. Unternehmen können sich somit für Anrufer eine bundesweite Präsenz verschaffen. Die Grundgebühr beträgt 2.000 DM/Monat und die Verkehrsgebühr -,23 DM/15 Sekunden.

Die 'Dezentrale Anrufweiterschaltung' (GEDAN) wird seit 1983 von der Deutschen Bundespost angeboten. GEDAN ermöglicht, mit Hilfe eines über einen Mikroprozessor gesteuerten 'Gerätes zur dezentralen Anrufweiterschaltung', die Erreichbarkeit an anderen orten, wobei die Rufnummer fest oder frei programmiert werden kann. Die Anschlußgebühr beträgt 2.000 DM, die Grundgebühr $166 \mathrm{DM} /$ Monat und die Verkehrsgebühr -,23 DM/15 Sekunden.

Der Dienst Konferenzgespräche wird über die Fernvermittlungsstelle Frankfurt nach Anmeldung aufgebaut und ermöglicht bis zu 15 Teilnehmern gleichzeitig, miteinander $z u$ konferieren.

16 Vgl. Oberpostdirektion Frankfurt: Leitfaden Telekommunikation Band 1, Fernmeldenetze und Dienste, Frankfurt 1986 , S. $7 \mathrm{ff}$.

17 Seit kurzem ist die Verbindung auch in das Ausland möglich. Langfristig soll dieser Dienst international angeboten werden.

18 Der Anrufende wird zum Ortstarif belastet und der Angerufene übernimmt die darüberhinaus anfallenden Kosten, in Form der Monatspauschale und zusätzlichen Gebühr. 
Der HauptanschluB mit Mehrfachzugang ermöglicht mehreren Anrufern gleichzeitig die Wiedergabe von Nachrichten, Bekanntmachungen, Werbespots usw.. Der Dienst wird seit Ende 1984 angeboten.

Voice-Mail ist eine Art 'elektronisches Postfach' bzw. 'elektronischer Briefkasten' für gesprochene Informationen. Ähnlich dem Prinzip eines Anrufbeantworters, werden Informationen gespeichert und abgerufen, wobei Voice-Mail zusätzliche Leistungsmerkmale bietet, wie die Zugangskontrolle mittels Paßwort, die Beantwortung, die Archivierung oder das Löschen. 19 Der Dienst befindet sich zur zeit noch in der Erprobungsphase.

\section{8prechfunk}

Der sogenannte bewegliche Landfunkdienst der Deutschen Bundespost ermöglicht Telefongespräche zwischen ortsfesten und beweglichen Landfunkstellen. Das Eurosignal20 - als Ergänzung - veranlaßt durch ein bestimmtes signal eine vereinbarte Reaktion, die in der Regel ein Rückruf bei der zentrale ist, um Informationen abzufragen und weitere Maßnahmen abzustimmen. Das Eurosignalgerät selbst kostet ca. 2.500 DM. Die monatlichen Gebühren belaufen sich auf 30 bis 50 DM/Monat. Das Funkfernsprechgerät ${ }^{21}$ liegt im Preis zwischen 5.000 und 10.000 DM, wobei sich mittlerweile ein Gebrauchtgerätemarkt entwickelt hat, auf dem Geräte ab ca. $2.000 \mathrm{DM} z u$ erhalten sind. Die Grundgebühr beträgt 120 DM/Monat und die Verkehrsgebühr,$- 23 \mathrm{DM} / 8$ sekunden. Mit Einführung des C-Netzes 1985 wurden die Leistungsmerkmale des Funktelefondienstes erheblich gesteigert.

19 Vgl. Ewers, H.; Fritsch, M.: Telematik und Raumentwicklung, a.a.o., s. 22 .

20 Auch Europiepser genannt.

21 In der Regel ein Autotelefon. 


\subsubsection{Daten- und Textkommunikation}

Datenkommunikation stellt im allgemeinen die nachrichtentechnische Ưbermittlung binär verschlüsselter Informationen zwischen zwei Endeinrichtungen mit der Möglichkeit der rechnergestützten Weiterverarbeitung dar. 22 Text- und Bildkommunikation gelten als Spezialformen der Datenkommunikation. Eine eindeutige zuordnung der Dienste zu den jeweiligen Kommunikationssammelbegriffen ist nicht möglich, da die Grenzen zwischen Text-, Daten- und Bildkommunikation fließend sind. Btx beispielsweise ermöglicht Text- und Datenkommunikation und einfache Bildkommunikation. Die hier vorgenommene Untergliederung in Daten- und Textkommunikation entspricht der Untergliederung der Dienste durch die Deutsche Bundespost. 23

\section{Datenkommunikation 24}

Die Datenübertragung mit $\operatorname{Modem}^{25}$, zur Wandlung digitaler signale in analoge, erfolgt über das Fernsprechnetz. Aufgrund der niedrigen Ubbertragungsgeschwindigkeit ${ }^{26}$ ist diese Kommunikationstechnik für kleinere Datenmengen prädestiniert. Der Dienst wird seit 1966 angeboten. Die Grundgebühr beträgt $27 \mathrm{DM} /$ Monat und die Anschlußgebühr 65 DM. Die Verkehrsgebühren entsprechen den Telefongebühren und sind somit entfernungs- und zeitabhängig.

22 Vgl. Ewers, H.; Fritsch, M.: Telematik und Raumentwicklung, a.a.0., s. 22 .

23 Vgl. Oberpostdirektion Frankfurt: Leitfaden Telekommunikation Band 1, a.a.0., S. 7 ff.

$24 \mathrm{Vgl}$. ebenda S. $31 \mathrm{ff}$.

25 Modulator/Demodulator.

26 Mit der bisherigen Technik sind Utbertragungsmengen von $4.800 \mathrm{bit} / \mathrm{s}$ und mit moderner Technik bis $9.600 \mathrm{bit} / \mathrm{s}$ möglich. Das entspricht $1 / 4$ bis $1 / 2$ DIN-A4-Seite pro Sekunde. 
Der seit 1967 angebotene Datex-I-Dienst ${ }^{27}$ ermöglicht einen schnellen Datenaustausch ${ }^{28}$ zwischen kompatiblen Datenendgeräten und zeichnet sich durch einen schnellen Verbindungsaufbau 29 und eine geringe Übertragungsfehlerquote aus. 30 Die Grundgebühr richtet sich nach der Übertragungsgeschwindigkeit und liegt zwischen 120 bis 2.000 DM/Monat. Die Verkehrsgebühr ist abhängig von der Verbindungsdauer, der Tageszeit, dem Wochentag und der Entfernung. Bundesweit nahmen Ende 1986 rund 18.000 Teilnehmer am Datex-L-Dienst teil.

Der seit 1981 angebotene Datex-P-Dienst ${ }^{31}$ dient der schnellen Datenübertragung ${ }^{32}$ zwischen Datenverarbeitungsanlagen. Die Datenübertragung ist im Unterschied zu Datex-L zwischen nicht kompatiblen Datenendgeräten ${ }^{33}$ möglich, wobei die Datenmenge in 'Pakete' zerlegt und paketweise, über Teilstrecken übermittelt wird. Die Grundgebühr beträgt zwischen 200 und 400 DM/Monat. Die Verkehrsgebühr ist abhängig von der Datenmenge und der Tageszeit. Datex-P ist kostenneutral bezüglich der Übertragungsentfernung. Bundesweit nahmen Ende 1986 rund 15.000 Teilnehmer am Datex-P-Dienst teil.

Für hohe Übertragungskapazitäten ${ }^{34}$ stehen seit 1973 fest-

27 data exchange, leitungsvermittelt.

28 Im Datex-L Netz sind Übertragungsgeschwindigkeiten von $50 \mathrm{bit} / \mathrm{s}$ bis zu $64.000 \mathrm{bit} / \mathrm{s}$ je nach Datenverarbeitungsanlage möglich.

29 Kleiner als eine sekunde.

30 Vgl. Schinagl, w.: Neue Dienste der Deutschen Bundespost, a.a.0., s. 3 .

31 data exchange, paketvermittelt.

32 Im Datex-P Netz sind Übertragungsgeschwindigkeiten von $300 \mathrm{bit} / \mathrm{s}$ bis zu $48.000 \mathrm{bit} / \mathrm{s}$ je nach Datenverarbeitungsanlage möglich.

33 Über Protokollanpassungen (gateways).

34 Im Direktrufnetz sind Übertragungsgeschwindigkeiten von $50 \mathrm{bit} / \mathrm{s}$ bis zu 1,92 $\mathrm{mbit} / \mathrm{s}$ je nach Datenverarbeitungs- 
geschaltete 'Standleitungen' im HfD-Netz ${ }^{35}$ zur Verfügung. Die Direktrufverbindungen ermöglichen gleichzeitiges Senden und Empfangen von Daten. Die Grundgebühr beträgt zwischen 60 und 400 DM/Monat. Die Verkehrsgebühr wird nach Entfernung, Übertragungsgeschwindigkeit und Nutzungszeit ermittelt. Ende 1986 waren bundesweit rund 140.000 Datenstationen angeschlossen.

Der 1985 eingeführte Telebox-Dienst stellt eine Art 'Elektronisches Postfach' dar und ist, neben dem Abspeichern von Mitteilungen, dem Anlegen von Verzeichnissen und der Kommunikation verschiedener Box-Adressen untereinander, in der Lage, Texte $\mathrm{zu}$ editieren und formatieren. International wird für die Dienstleistung Telebox auch der Sammelbegriff 'Mailbox' oder 'Electronic Mail' verwendet. Die Telebox kann, mittels eines Paßwortes, von jedem ort aus über das Fernsprech- oder Datexnetz angeschaltet werden. Die Anschlußgebühr beträgt 65 DM und die Grundgebühr 40 DM/Monat. Zusätzlich fallen zeitabhängige Nutzungsgebühren von 0,30 DM/Minute, Adressiergebühren und Übermittlungsgebühren an. 361987 waren bundesweit ca. 400 Teilnehmer angeschlossen.

Der Temex ${ }^{37}$-Dienst befindet sich zur zeit in der Pilotphase und soll 1991 flächendeckend angeboten werden. Temex dient der Übertragung von sogenannten Fernwirkinformationen, wie Fern-Anzeigen, Fern-Messen, Fern-Schalten und Fern-Einstellen. Der Austausch der Fernwirkinformationen erfolgt über das Fernsprechnetz, wobei die gleichzeitige Nutzung des Telefon-Anschlusses für das Fernsprechen nicht beeinträchtigt

anlage möglich.

35 ㅌaptanschluß für Direktruf.

$36 \mathrm{Vgl}$. Deutsche Bundespost: Telebox - Das Angebot der Deutschen Bundespost für Mitteilungen von Person zu Person, 0.0., Januar 1989.

37 telemetry exchange. 
wird. Die Kosten für Standard-Endgeräte liegen zwischen 100 und 300 DM und für die Leitstellen ab 5.000 DM aufwärts. Die Grundgebühr ist nutzungsabhängig und beträgt für den Taxiruf $15 \mathrm{DM} /$ Monat für bis zu 2.000 Rufe. 38

\section{Textkommunikation 39}

Der bereits seit 1933 existierende Telex-Dienst 40 ubbermittelt preiswert, dafür relativ langsam 41 und mit eingeschränktem Zeichenvorrat, Texte mit Hilfe von Fernschreibmaschinen. Aufgrund genormter technischer Vorkehrungen, gelten die Texte die übertragen werden als rechtsverbindlich. Telex ist kompatibel zum Teletex-, Telegramm-, Btxund Telebox-Dienst. Der für das Telex notwendige Bürofernschreiber kostet bis $z u \quad 60.000$ DM. Die Grundgebühr beträgt $80 \mathrm{DM} /$ Monat und die Verbindungsgebühr 0,10 DM/15 Sekunden. 1988 waren bundesweit ca. 180.000 und international mehr als 1,7 Millionen Teilnehmer angeschlossen. ${ }^{42}$

Der seit 1981 zur Verfügung stehende Teletex-Dienst stellt eine leistungsfähigere Form des Telex-Dienstes mit höherer Übertragungsgeschwindigkeit 43 und zusätzlichen Textverarbeitungsfunktionen dar. Teletex ermöglicht format- und layoutfreie Textkommunikation, die mit einer multifunktionalen speicherschreibmaschine, Textverarbeitungsanlagen

$38 \mathrm{Vgl}$. Funke + Huster Elektrizitätsgesellschaft $\mathrm{mbH} \& \mathrm{Co}$. KG (Hrsg.): Der neue Temex-Service der Bundespost, in: Frankfurter Allgemeine Zeitung Nr. 7 vom 9. Januar 1990, S. 16 .

39 Vgl. Oberpostdirektion Frankfurt: Leitfaden Telekommunikation Band 1, a.a.0., s. $40 \mathrm{ff}$.

40 Gleich Fernschreiben.

41 Übertragungsgeschwindigkeit 400 zeichen in 60 Sekunden. Die Übertragung einer DIN-A4-Seite dauert ca. 5 Minuten.

42 Vgl. Deutsche Bundespost: Dienste der Deutschen Bundespost für die Textkommunikation, 0.0., April 1989.

43 Die Utbertragungsgeschwindigkeit im Teletex beträgt 2.400 bit/s, im Gegensatz zu Telex mit 50 bit/s. Die Übertragungszeit für eine DIN-A4-Seite liegt bei 10 Sekunden. 
oder anderen Techniken, wie Personal Computern, durchgeführt wird, wobei die Geräte für den normalen Schreibbetrieb verwendet werden können. Teletex ist kompatibel zum Telex-, Telegramm- und Cityruf-Dienst. Die Teletexgeräte kosten zwischen 5.000 und 15.000 DM, die Grundgebühr 170 $\mathrm{DM} /$ Monat $+3 \mathrm{Pf} /$ Verbindung und die Verbindungsgebühr von 0.97 bis $1.65 \mathrm{Pf} / \mathrm{Sek}$. Bundesweit nutzten 1988 rund 20.000 Teilnehmer mit mehr als 30.000 Endeinrichtungen diesen Dienst.

Btx $^{44}$ ist ein kombinierter Dienst zur Übermittlung von Text, einfachen Grafiken und Daten, der seit 1983 angeboten wird. Anschlußmöglichkeiten existieren als Anbieter oder Teilnehmer. Die Informationen können visuell auf einem Bildschirm dargestellt, ausgedruckt, gespeichert und direkt weiterverarbeitet werden. Bildschirmtext ist ein Medium, das besonders preiswert ist und sich vor allem für die Kommunikation mit vielen externen Teilnehmern eignet. Zusätzlich bietet Btx die Möglichkeit sogenannter geschlossener Benutzer-Gruppen. 45 Das Empfangsgerät mit Decoder und Tastatur kostet ca. 3.000 DM. Die Grundgebühr beträgt 8 DM/Monat und die Verkehrsgebühr entspricht der Telefongebühr im Nahbereich. Bundesweit hat der Btx-Dienst 1989 rund 180.000 Anschlüsse, die zu zweidrittel geschäftlich genutzt werden.

Die zukünftige Entwicklung des Btx-Dienstes ist zur zeit nicht absehbar. Die unter den Schlagworten 'tele-shopping', 'home-banking' und 'tele-arbeit' bekannten Nutzungen sind bis heute noch nicht in der geplanten Breite akzeptiert worden. Neue Perspektiven für gewerbliche und private Nutzungen können jedoch durch die verbesserten Leistungsmerk-

\section{Biildschirmtex̧t}

$45 \mathrm{Vgl}$. Schmidt, B.: Bildschirmtext ist immer noch kein Medium für den privaten Markt, in: Frankfurter Allgemeine Zeitung $\mathrm{Nr} .230$ vom 4. Oktober 1989, S. 22. 
male mit Einführung des ISDN entstehen. 46

\subsubsection{Bildkommunikation}

Die durch die Deutsche Bundespost angebotenen Bildkommunikationsdienste untergliedern sich in Fest- und Bewegtbildkommunikation. Die Festbildkommunikation umfaßt die Nutzungsformen Telefax, Einzelbildübertragung und Fernzeichen, die Bewegtbildkommunikation das Bildtelefon und die Videokonferenz. Prinzipiell ergeben sich durch den Einsatz der Bewegtbildkommunikation eine Vielzahl von Anwendungsmöglichkeiten. Aufgrund der notwendigen, relativ hohen Übertragungskapazität, setzt der Einsatz von Bewegtbildkommunikation die Existenz von Breitbandnetzen voraus.

\section{Festbildkommunikation 47}

Seit 1979 werden im Telefax-Dienst Schrift und Grafiken originalgetreu 48 übermittelt. Die Grundausstattung setzt sich aus einem Fernkopierer, einer Anschalteeinrichtung und einem Telefonanschluß zusammen. In Abhängigkeit von der Gruppe des Fernkopierers, werden drei Minuten oder eine Minute für das Kopieren einer DIN-A4-Seite benötigt. Die momentanen Mängel bezüglich der Utbertragungszeit, Farbübertragung und Übertragung flächiger Bilder werden mit Anwendung der Digitaltechnik weitgehend beseitigt. Als zusätzlichen Service bietet die Deutsche Bundespost, für Kunden ohne eigenes Telefax-Gerät, in etwa 600 Postämtern innerhalb der Bundesrepublik Deutschland, öffentliche Fernkopierer an. Die Kosten für einfache Endgeräte liegen mittlerweile unter $2.000 \mathrm{DM}$. Neben der Bereitstellungsgebühr

46 Vgl. Ewers, H.; Fritsch, M.: Telematik und Raumentwicklung, a.a.0., S. 24.

$47 \mathrm{Vgl}$. Oberpostdirektion Frankfurt: Leitfaden Telekommunikation Band 1, a.a.0., S. 90 ff.

48 Zur zeit ausschließlich in schwarz/weiß. 
von 65 DM, fällt die Gebühr für den zusätzlichen Telefonanschluß, in Höhe von 20 bis 27 DM/Monat, die Grundgebühr von 8 DM/Monat und die Verkehrsgebühr, in Höhe der Verbindungsgebühren für den Telefondienst, an. Bundesweit waren per 30. Dezember 1988197.245 Teilnehmer angeschlossen. 49

Im Einzelbildkommunikations-Dienst erfolgt die Wiedergabe der Festbilder in der Regel über den Bildschirm. Das original wird mit einer Kamera oder einem scanner aufgenommen, gespeichert und aus diesem Speicher übertragen. Die Telekommunikation mit sogenannten 'Festbildfolgen' benötigt mit der Analogtechnik ca. 50-60 Sekunden pro Bild und reduziert sich bei Einführung des schmalbandigen ISDN-Netzes auf ca. vier Sekunden pro Farbbild. 50 Die Kosten und der Einführungszeitpunkt sind momentan nicht absehbar.

Eine Zwischenform von Fest- und Bewegtbildkommunikation stellt der Fernzeichen-Dienst dar. Skizzen und Schriftzüge können während des zeichenvorgangs mit Hilfe eines stiftes auf einer Halbleiterplatte oder mit normaler Kreide auf einer elektronischen Tafel übertragen werden. Die Wiedergabe erfolgt auf einem Bildschirm. Nutzungsmöglichkeiten existieren vor allem in Verbindung mit der Sprachkommunikation. Kosten und Einführungszeitpunkt sind noch nicht absehbar. 51

Bewegtbildkommunikation 52

Unter dem Bildtelefon-bzw. Bildfernsprech-Dienst wird die Erweiterung des Telefonierens um die Bewegtbildübertragung

49 Vgl. O.V.: Textkommunikation per Telefax und Teletex, in: Deutsche Handwerks Zeitung Nr. 11 vom 9. Juni 1989, Seite 17.

50 Vgl. Ewers, H.; Fritsch, M.: Telematik und Raumentwicklung, a.a.o., s. 26 .

$51 \mathrm{Vgl}$. ebenda S. $26 \mathrm{f}$.

52 Vgl. Oberpostdirektion Frankfurt: Leitfaden Telekommunikation Band 1, a.a.0., S. 90 ff. 
verstanden. Die Bildqualität richtet sich nach dem verwendeten Fernseher und kann in Farbe und normaler zeitlicher und räumlicher Auflösung übertragen werden. Zusätzlich besteht die Möglichkeit, auf Vereinbarung das Bild ab- und zuzuschalten. Der Normalbetrieb soll Ende 1990 aufgenommen werden, wobei die Kosten noch nicht definitiv festgelegt sind. 53

Der seit 1985 im Testbetrieb befindliche videokonferenzDienst stellt eine Anwendungsform des Bildfernsprechens dar. Videokonferenzen verbinden Personengruppen zu gemeinschaftlichen Besprechungen mit Bild und Ton. Zusätzlich zur Bewegtbildkommunikation in Farbe, werden Leistungsmerkmale wie Dokumenten-, Text-, Daten- und Grafikkommunikation angeboten, die parallel zur Videokommunikation übertragen werden. 54 Das, zunächst auf die verbindung von zwei videokonferenzräumen konzeptionierte, Versuchsnetz, bietet seit 1989 die Option von Multipointkonferenzschaltungen. Der Videokonferenzdienst kann über öffentliche Videokonferenzräume oder unternehmenseigene Einrichtungen genutzt werden. 1988 waren bundesweit 66 private una 13 öffentliche videokonferenzräume am Netz.

Eine Videokonferenzanlage, die vom Teilnehmer selbst eingerichtet wird, kostet zwischen ca. 100.000 und 500.000 DM, zuzüglich einer einmaligen Anschlußgebühr von 12.000 DM, der Grundgebühr von 1.500 DM/Monat und der Verkehrsgebühr zwischen 600 DM/Stunde innerhalb der Bundesrepublik Deutschland und 2.500 DM/Stunde, beispielsweise von der Bundesrepublik Deutschland in die USA. Für die Nutzung öffentlicher videokonferenzräume wird zusätzlich ein Nut-

$53 \mathrm{Vgl.}$ Fröhlich, R.: Von Angesicht zu Angesicht - Bildtelefon geht Ende des Jahres in Betrieb, in: Frankfurter Allgemeine Zeitung Nr. 67 vom 20. März 1990, S. B5.

$54 \mathrm{Vgl}$. Deutsche Bundespost: Mit Videokommunikation in die Zukunft blicken, in: Videokommunikation visuell 1/1988, S. 2 . 
zungsentgelt von $120 \mathrm{DM} /$ Stunde erhoben. 55

\subsection{Entwicklungsstrukturen der Telematik}

\subsubsection{Erwarteter Telekommunikationsbedarf in Hessen}

\subsubsection{Einführung}

Aufgrund der Imponderabilien der wirkungen des Einsatzes neuer telekommunikativer Techniken, erscheint die Frage nach den Größenordnungen des zukünftigen Einsatzes der Telekommunikationstechniken besonders relevant. Für die Entwicklung des qualitativen Nachfragepotentials nach neuen telekommunikativen Techniken ist die bisherige quantitative Nachfrageentwicklung von besonderer Bedeutung. Das bedeutet, die Agglomerationen der momentan angebotenen Telekommunikationsdienste stellen primär die größten Nachfragepotentiale für neue Dienstleistungen dar. 56

Bei der Analyse der Ergebnisse diesbezüglicher Bedarfsuntersuchungen ist $z u$ berücksichtigen, daß die Daten nur begrenzte Aussagen über die tatsächliche Nachfrageentwicklung liefern können. Die Ursache hierfür liegt darin, daß die Anwender teilweise nur geringe Kenntnisse über die neuen telekommunikativen Techniken besitzen. Dies gilt im besonderen für Bedarfsanalysen von noch nicht angebotenen Diensten.

$55 \mathrm{Vgl}$. Deutsche Bundespost: Videokonferenz/Videokommunikation - Entgelte -, 0.0., stand 1989.

56 Vgl. Schiffer, R.: Konzeption der Telekommunikationsnetze und Planung für das Land Rheinland-Pfalz, a.a.o., s. 2 . 
Anschließend an die überblickartige Darstellung der empirischen Analyse der Gesellschaft für Information und Dokumentation, wird die Prognose der oberpostdirektion Frankfurt am Main zur 'ISDN-Kundenverteilung in Hessen' dargestellt. 57

\subsubsection{Empirische Befunde der Gesellschaft für Informa- tion und Dokumentation}

Die folgenden empirischen Befunde basieren auf einer Bedarfsanalyse der Gesellschaft für Information und Dokumentation, die vom Hessischen Minister für wirtschaft und Technik in Auftrag gegeben wurde. Die studie konzentriert sich auf den Bereich Individual-Kommunikation für den Geschäftsbedarf und umfaßt den Zeitraum von 1985 - 2005.58

Bei der Analyse der längerfristigen Bedarfsentwicklung, die mittels eines Experten-Interviews erfolgte, kristallisierte sich die Tendenz einer Verschiebung, im Bereich der künftigen Nutzung einzelner Telekommunikationsdienste, heraus.59 Tabelle 1.1 stellt die Substitutionsabsichten für die Dienste Briefpost, Fernsprechen und Telex durch neue Telekommunikations-Dienste dar.

Der Briefpost- und der Telex-Dienst werden in etwa gleich stark durch die neuen telekommunikativen Techniken substituiert. Als substitutionsauslösender Faktor wurde primär der Kostenfaktor genannt.

57 Vgl. Oberpostdirektion Frankfurt: Leitfaden Telekommunikation, Ergänzung $\mathrm{zu}$ Band 2, Frankfurt 1986, S. 18 f.

$58 \mathrm{Vgl}$. Winand, U. u.a.: Telekommunikation in Hessen, Gesellschaft für Information und Dokumentation mbH (Hrsg.), Frankfurt 1986.

$59 \mathrm{Vgl}$. ebenda S. 26. 
Tabelle 1.1: Substitutionstendenzen 60

\begin{tabular}{|l|c|c|c|}
\hline & Briefpost & Fernsprechen & Telex \\
\hline \hline wird teilweise ersetzt durch: & 3 & - \\
\hline Telex & 17 & 4 & 101 \\
\hline Teletex & 44 & 3 & 2 \\
\hline Btx & 23 & 2 & 5 \\
\hline Telebox & 18 & 1 & 17 \\
\hline $\begin{array}{l}\text { Datenübertra- } \\
\text { gungsdienste }\end{array}$ & 30 & 3 & 55 \\
\hline Telefax & 29 & \multicolumn{2}{|c|}{3} \\
\hline Anzahl der Nennungen von 284 Befragten
\end{tabular}

Während die Briefpost durchgängig von allen Telekommunikationsdiensten relativ gleichmäßig substituiert wird, wird Telex primär durch Teletex und durch Telefax substituiert. Beim Fernsprechen besteht nahezu kein substitutionspotential. Auf lange sicht gesehen wird das Fernsprechen nicht durch andere Dienste ersetzt werden. Unter Berücksichtigung aller Telekommunikationsdienste, wird das Fernsprechen jedoch einen abnehmenden Anteil am Telekommunikations-Gesamtaufkommen verzeichnen. 61

Abbildung 1.3 stellt eine Schätzung der Entwicklung des Übertragungsvolumens der Sprach-, Brief-, Text- und DatenDienste bis zum Jahr 2005 dar. Als Mengenbasis für die Abbildung 1.3 wurde der Indexwert 100 für das Jahr 1984 gewählt.

60 In Anlehnung an winand, U. U.a.: Telekommunikation in Hessen, a.a.0., s. 28 .

61 Siehe auch Abbildung 1.3. 
Die Basiswerte für 1984 waren im einzelnen: ${ }^{62}$

- Sprachübertragung:

- Briefpost:

- Elektronische Textübertragung:

- Datenübertragung: ca. 2,7 Mrd. Gespräche in 1984

ca. 1,3 Mrd. Briefe in 1984

ca. 39 Gbit/Tag

ca. 1253 Gbit/Tag

Abbildung 1.3: Schätzung des zukünftigen Übertragungsvolumens 63

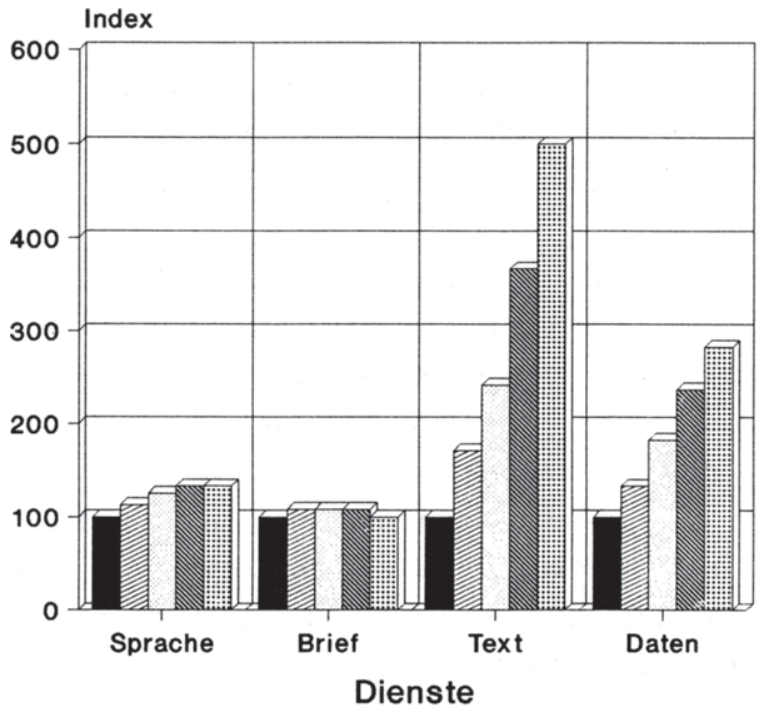

— $1984 \square 1990 \quad \square 1995$

62 Vgl. Winand, U. u.a.: Telekommunikation in Hessen, a.a.o., s. 32 .

63 In Anlehnung an Winand, U. U.a.: Telekommunikation in Hessen, a.a.0., S. 32 . 
Nach der Schätzung des zukünftigen Übertragungsvolumens werden sich die Sprach-, Brief-, Text- und Datendienste in den nächsten 15 Jahren folgendermaßen entwickeln:

- Das Übertragungsvolumen der Sprachkommunikationsdienste wird bis zum Jahr 2005 lediglich um 20 Prozent ansteigen.

- Der Briefpost-Dienst wird in etwa auf dem heutigen Niveau verharren und langfristig eher zurückgehen.

- Das Übertragungsvolumen der Textübertragungs-Dienste wird um das 3,5-fache anwachsen.

- Das Übertragungsvolumen der Datenübertragungs-Dienste wird sich in etwa verdoppeln.

Bei der Bildkommunikation wird ein Bedeutungszuwachs erwartet, der sich momentan jedoch nicht spezifizieren läßt, da diesbezügliche schätzungen stark divergieren.

Die erwartete Bedarfsentwicklung stellt sich nicht homogen über die wirschaftssektoren hin dar, sondern verteilt sich unregelmäßig über die verschiedenen Branchen ${ }^{64}$. Wie aus Tabelle 1.2 ersichtlich, bestehen starke sektorale Differenzen bezüglich der 'Nutzung', 'Geplanten Nutzung' und dem 'Interesse' an Telekommunikationsdiensten, wobei sich einige sektoren auf spezielle Telekommunikationsdienste konzentriert haben und in diesen Bereichen eine eindeutige spitzenstellung einnehmen. 65

64 Hier Sektoren genannt.

65 Hierbei steht ' + ' für den höchsten und '-' für den niedrigsten Anteil an 'Nutzung', 'Planung' und 'Interesse' an den aufgeführten Telekommunikationsdiensten. 
Tabelle 1.2: Nutzung von und Interesse an Telekommunikationstionsdiensten nach Sektoren 66

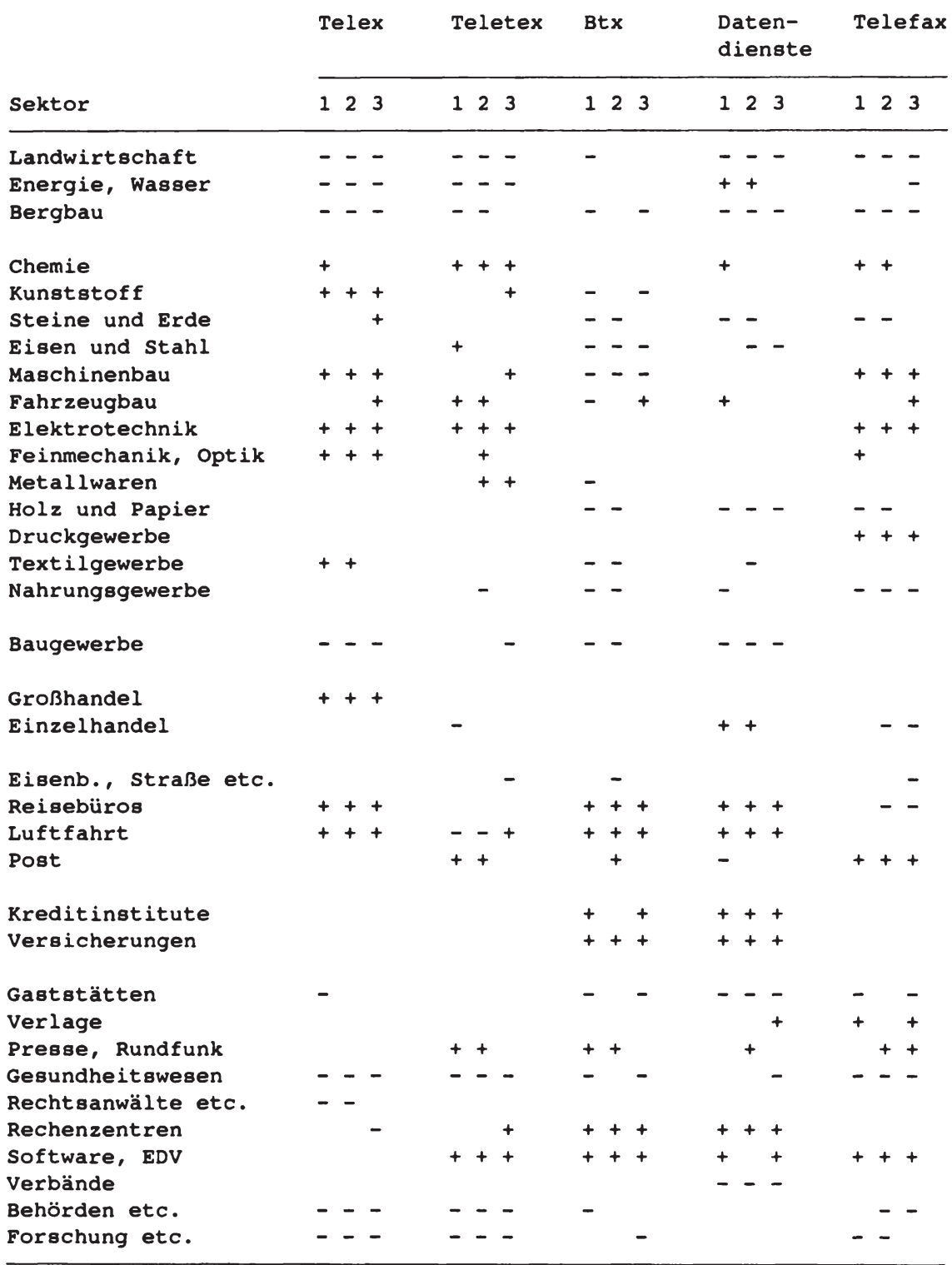

1 = genutzt, 2 = genutzt oder geplant, 3 = mindestens Interesse

Lediglich geringe Divergenzen der erwarteten Bedarfsent-

66 In Anlehnung an winand, U. u.a.: Telekommunikation in Hessen, a.a.0., s. 35 . 
wicklung stellen sich in Abhängigkeit der Raumordnungsregionen ein. Bei der Analyse des Bedarfs an neuen telekommunikativen Techniken, nach den Raumordnungsregionen Nordhessen, Mittelhessen, Osthessen, Rhein-Main-Taunus, Untermain und starkenburg, konnte keine eindeutige Abhängigkeit des Telekommunikationsbedarfs von der Raumordnungszugehörigkeit festgestellt werden, da die Ergebnisse in den sechs Regionen nur geringfügig von den jeweiligen Anteilswerten für Gesamthessen abweichen. Den niedrigsten Bedarf nach Datenübertragungsdiensten und Telefax weist die Region Osthessen auf. Die Region Untermain 67 liegt bei den meisten Diensten an der spitze, muß diesen Platz jedoch, mit Ausnahme von Telefax, mit anderen Regionen teilen. 68

Die Ergebnisse der Analyse nach Raumordnungsregionen, spiegeln das vorhandene Nord-Süd-Gefälle in Hessen nicht wie erwartet wieder, sondern lassen eher geringe Unterschiede vermuten. Ursache hierfür könnte der fehlende zeitliche Bezugsrahmen der Befragung sein, der besonders im Bereich des 'Interesses' einen großen Spielraum offenläßt.

Dieser Sachverhalt wird durch die Delphi-Befragung, bezüglich der Zeitpunkte, in denen ein 5\%-bzw. 10\%-Anteil an ISDN-Hauptanschlüssen in den hessischen Regionen erwartet wird, dokumentiert. Für die Region Rhein-Main-Taunus wird bereits für 1992 mit einem 10\%-Anteil gerechnet, gegenüber Nordhessen, Mittelhessen und Osthessen, welche erst im Jahre 2000 den $10 \%$-Anteil erreichen werden. 69

67 Einschließlich dem Großraum Frankfurt.

$68 \mathrm{Vgl}$. Winand, U. u.a.: Telekommunikation in Hessen, a.a.0., s. 50 .

$69 \mathrm{Vgl}$. ebenda. 


\subsubsection{Prognose der Deutschen Bundespost}

Die Prognose der Deutschen Bundespost dokumentiert den erwarteten Bedarf an Telekommunikationsdiensten im Rahmen des ISDN bis zum Jahre 1991.70

Basis der Prognose war die struktur und die regionale Verteilung der Teilnehmer an den Fernsprech-, Text- und Datendiensten zum 31.12.1985 und die Annahme, daß Fernsprechteilnehmer, insbesondere mit großen Nebenstellenanlagen, aufgrund wirtschaftlicher Erwägungen die neuen Telekommunikationsdienste verstärkt nutzen werden. Eine weitere berücksichtigte Interessengruppe waren die Anbieter im BtxDienst. Die Interessengruppe der Teilnehmer an Text- und Datendiensten wurde in zwei Kategorien aufgeteilt:

- die Nutzer von Datex-L, Datex-P und HfD und

- die Nutzer von Telex und Teletex.

Ausgehend von diesen Parametern, wurde eine Dichteskala der potentiellen ISDN-Kunden in den verschiedenen Fernsprechortsnetzen entwickelt, die die regionale Verteilung des Bedarfs anhand von vier Kategorien wiedergibt. Die Dichteskala wurde mathematisch über den Dichtefaktor Delta ermittelt.

Delta $=\frac{\text { Geschätzte Anzahl der erforderlichen Anschlußleitungen }}{\text { Geschätzte Anzahl der potentiellen ISDN-Kunden }}$

Hierbei wird unter 'Anschlußleitungen' die Teilnehmeranschlußleitungen für das Übermitteln digitaler Informationen mit einer Nettobitrate von $144 \mathrm{kbit} / \mathrm{s}$ verstanden. 71

$70 \mathrm{Vgl}$. Oberpostdirektion Frankfurt: Leitfaden Telekommunikation, Ergänzung $\mathrm{zu}$ Band 2, a.a.0., S. $18 \mathrm{f}$.

$71 \mathrm{Vgl}$. ebenda. 
Die prognostizierte ISDN-Kundenverteilung der Deutschen Bundespost weist für Hessen einen 'besonders hohen Bedarf'72 ausschlieBlich für den Bereich Frankfurt aus. Frankfurt selbst ist zusätzlich von Fernsprechortsnetzen mit überwiegend 'hohem Bedarf'76 umgeben. Gießen, Kassel und Fulda dokumentieren einheitlich einen 'hohen Bedarf'76. Unterschiede existieren jedoch im Umfeld der drei städte. Während Gießen und Fulda jeweils von drei Ortsnetzen mit 'hohem Bedarf'76 umgeben sind, hat Kassel lediglich ein benachbartes ortsnetz mit 'mittlerem Bedarf'76. Kassel nimmt für Nordhessen somit quasi eine Inselstellung im Bereich der prognostizierten ISDN-Kundenverteilung ein. 73

Die Prognose der Deutschen Bundespost signalisiert insgesamt ein eindeutiges Süd-Nord-Gefälle im Bereich der ISDNKundenverteilung für das Land Hessen, wobei der Nachfrageschwerpunkt auf das Rhein-Main-Gebiet konzentriert ist und über die Regionen Gießen und Fulda nach Norden hin immer stärker abnimmt.

\subsubsection{Gegenwärtige Infrastruktur und Agglomeration der Telekommunikation}

\subsubsection{Infrastuktur der Bundesrepublik Deutschland}

Basis der individuellen Telekommunikation, im Rahmen der durch die Deutsche Bundespost angebotenen Dienste, stellen

72 Prognostizierte ISDN-Kundenverteilung nach Gruppen:

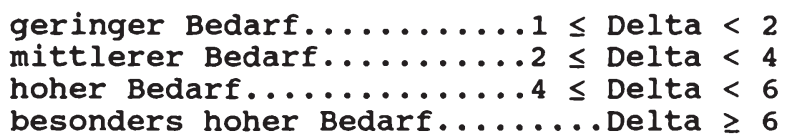

$73 \mathrm{Vgl}$. Oberpostdirektion Frankfurt: Leitfaden Telekommunikation, Ergänzung zu Band 2, a.a.0., S. $18 \mathrm{f}$. 
die, in der Regel verkabelten, Fernmeldenetze dar. Die Satellitenübertragung ist primär dem internationalen Verkehr vorbehalten. Die dominierenden Parameter der Fernmeldeinfrastruktur stellen die übertragungskapazität der Leitungen und die Leistungsfähigkeit der Vermittlungsstellen und Endeinrichtungen dar. Die Kapazitäten dieser Parameter determinieren die potentiellen Telekommunikations-Anwendungen.

Die für die Telekommunikation relevanten öffentlichen Netze sind:

- das Fernsprechnetz,

- das integrierte Text- und Datennetz und

- das Vorläufer-Breitband-Netz. 74

Das lokale Breitbandverteilnetz aus Kupferkoaxialkabel dient der Übertragung von Ton- und Fernsehrundfunkprogrammen, im Bereich der hier nicht behandelten Massenkommunikation.

\section{Fernsprechnetz}

Mit über 26 Millionen Hauptanschlüssen und über 30 Millionen Telefon-Endgeräten, kann das Fernspechnetz als flächendeckend angesehen werden. Das Fernsprechnetz ist ein weitgehend analoges, schmalbandiges Vermittlungsnetz, das aus Kupferdoppeladern aufgebaut ist. Seit 1982 wird im Bereich des regionalen Fernverkehrs die Umstellung vom Analog- auf den Digitalbetrieb vorgenommen. 75

Zusätzlich zum Telefondienst, werden die Dienste Telefax, Btx, sowie ca. ein Drittel der Datenfernübertragungsdienste über das Fernsprechnetz abgewickelt.

74 Vgl. Bundesministerium für das Post- und Fermeldewesen: Mittelfristiges Programm für den Ausbau der technischen Kommunikationssysteme, Bonn 0.J., S. 12.

$75 \mathrm{Vgl}$. Oberpostdirektion Frankfurt: Leitfaden Telekommunikation Band 1, a.a.0., S. $8 \mathrm{ff}$. 


\section{Integriertes Text- und Datennetz}

Das integrierte Text- und Datennetz (IDN) ist ein digitales Fernmeldenetz mit digitaler Übertragungs- und Vermittlungstechnik. Es wurde 1976 von der Deutschen Bundespost aufgebaut und umfaßt, neben Telex und Teletex, die Datenübertragungsdienste Datex-L, Datex-P und die sogenannten 'standleitungen' des HfD-Netzes. Im IDN erhält der Anwender für jeden Dienst einen eigenen Anschluß. 76

\section{Vorläufer-Breitband-Netz}

Das Vorläufer-Breitband-Netz (VBN) ist als GlasfaserOverlay-Netz ausgelegt. Abbildung 1.4 stellt das VorläuferBreitband-Netz zum Ausbauzustand Ende 1988 dar, in dem 29 zentrale städte der Bundesrepublik Deutschland miteinander verbunden sind.

"Unter einem Glasfaser-Overlaynetz versteht man ein dem bestehenden Telefonnetz überlagertes, aber zunächst von diesem unabhängigen Glasfasernetz von Teilnehmer zu Teilnehmer, das den erst allmählich wachsenden Bedarf an breitbandigen Diensten der Individualkommunikation abdecken sol1."77 Es nutzt die Infrastruktur des bestehenden Telefonnetzes $^{78}$ und wird seit 1986 ausgebaut. Ziel der Ausbaustrategie ist es, "mit Probeangeboten auf den Markt zu gehen und dergestalt die Möglichkeit dafür zu schaffen, daß sich eine neue Generation von Telekommunikationsdiensten vom Markt her und im verstärkten Dialog zwischen Anwendern und der DBP entwickeln kann"79.

76 Vgl. Haist, W.: Weiterentwicklung der Fermeldesysteme, in: Elias, D. (Hrsg.): Telekommunikation in der Bundesrepublik Deutschland 1982, Heidelberg 1982, S. 156.

77 Oberpostdirektion Frankfurt: Leitfaden Telekommunikation Band 1, a.a.0., S. 80.

78 Wie Kabelkanäle und Fernmeldedienstgebäude.

79 Minister für Wirtschaft, Mittelstand und Technologie des Landes Nordrhein-Westfalen und Deutsche Bundespost (Hrsg.): Telekommunikations-Atlas Nordrhein-Westfalen Band 2, 0.0. September 1988, S. 54 . 
Abbildung 1.4: Vorläufer-Breitband-Net $z^{80}$

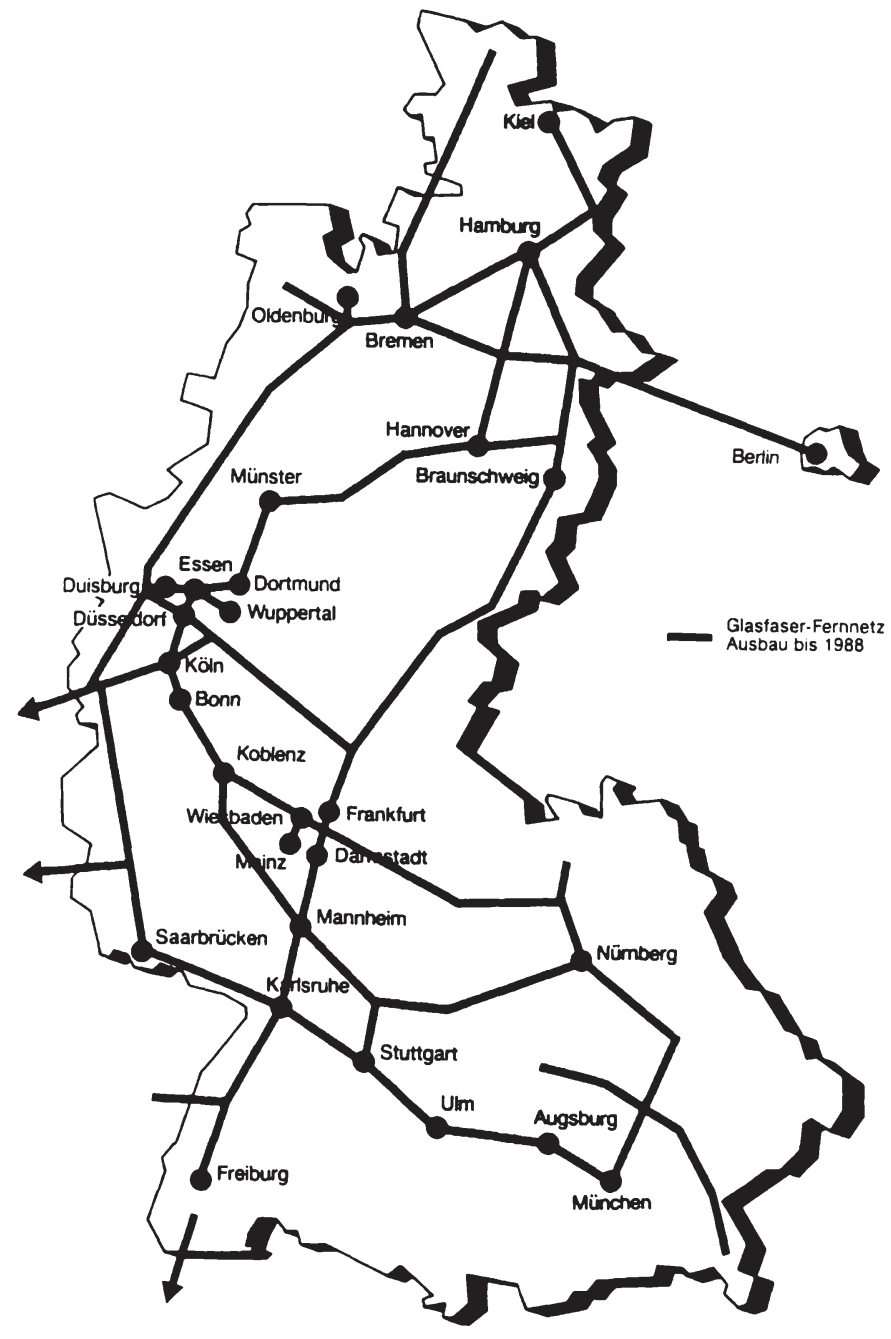

80 Quelle: Deutsche Bundespost: MEDKOM ein Anwendungsprojekt zur Erprobung der medizinischen Kommunikation im Vorläufer-Breitband-Netz VBN, 0.0. 0.J.. 
Die neben den öffentlichen Telekommunikationsnetzen existierenden privaten 'Inhouse-Netze' erfreuen sich zunehmender verbreitung. 81

\subsubsection{Agglomerationen in Hessen}

Die Agglomerationen der Telekommunikationsdienste in Hessen werden im folgenden skizziert. Sie geben einen Überblick über die jeweiligen Agglomerationen der entsprechenden Telekommunikationsdienste in Hessen, wobei das existierende Nord-Süd-Gefälle in der Anschlußdichteverteilung der verschiedenen Nutzungen deutlich hervortritt.

\section{Verteilung der Fernspechhauptanschlüsse}

Die Verteilung der geschalteten Fernsprechhauptanschlüsse auf die einzelnen Fernsprechortsnetze in Hessen wurde von der Deutschen Bundespost zum 31.12.1985 erhoben. Die ortsnetze wurden, entsprechend der summe der Anschlüsse, in sechs Größenklassen eingeteilt. 82

Das Fernsprechortsnetz mit den meisten Fernsprechhauptanschlüssen stellt Frankfurt am Main, mit mehr als 300.000 Anschlüssen, dar und ist primär von ortsnetzen mit 30.000 bis 299.999 Anschlüssen und 10.000 bis 29.999 Anschlüssen umgeben. Besonders deutlich wird die Diskrepanz zu Nordund Osthessen, wo lediglich Kassel und Fulda als 'Telekommunikations-Inseln' mit 30.000 bis 299.999 Fernsprechhauptanschlüssen herausragen. Dieser Effekt wird zusätzlich durch die umliegenden ortsnetze verstärkt, die auf erheblich niedrigerem Niveau liegen. Kassel ist primär von ortsnetzen mit 3.000 bis 9.999 Fernsprechhauptanschlüssen umge-

81 Vgl. Ewers, H.; Fritsch, M.: Telematik und Raumentwicklung, a.a.0., S. 27.

82 Vgl. Oberpostdirektion Frankfurt: Leitfaden Telekommunikation, Ergänzung zu Band 2, a.a.0., S. 8 . 
ben und Fulda primär von Ortsnetzen mit 1.000 bis 2.999 Anschlüssen.

\section{verteilung der Text- und Datenanschlüsse}

Die Verteilung der geschalteten Text- und Datenanschlüsse auf die einzelnen Fernsprechortsnetze in Hessen wurde von der Deutschen Bundespost ebenso zum 31.12.1985 erhoben. Basis der Erfassung waren die Anschlüsse der folgenden Dienste:

- Textdienste: Telex, Teletex, Telefax und Btx und

- Datendienste: Datex-L, Datex-P, HfD und Datenübertragung im Fernsprechnetz. 83

Bei der Erfassung wurden die unterschiedlichen Übertragungsgeschwindigkeiten von $50 \mathrm{bit} / \mathrm{s}$ bis $48.000 \mathrm{bit} / \mathrm{s} \mathrm{nicht}$ berücksichtigt. Die Text- und Datenanschlüsse wurden somit linear addiert. Die Einteilung der ortsnetze in die entsprechende Größenklasse erfolgte nach der Anzahl der am stichtag geschalteten Text- und Datenanschlüsse.

Das Fernsprechortsnetz mit den meisten Text- und Datenanschlüssen stellt Frankfurt am Main, mit ca. 24.000 Anschlüssen, dar. Es ist primär von ortsnetzen mit 101 bis 600 Anschlüssen umgeben. Im Vergleich zur Verteilung der Fernsprechhauptanschlüsse, ist die Verteilung der Text- und Datenanschlüsse noch stärker auf die Agglomeration RheinMain und die umliegenden Fernsprechortsnetze konzentriert. Auch hier wird die Diskrepanz zu Nord- und Osthessen deutlich, wo lediglich Kassel und Fulda, mit jeweils 601 bis 3.000 Anschlüssen, herausragen. Auffallend ist das Gefälle zwischen dem ortsnetz Kassel und den umliegenden ortsnetzen, mit primär lediglich 11 bis 30 Anschlüssen. Im Vergleich der umgebenden ortsnetze schneidet Fulda, mit einem Ortsnetz der Gruppe 101 bis 600 Anschlüsse, besser als Kassel ab.

$83 \mathrm{Vgl}$. ebenda S. 10 . 


\subsubsection{I8DN-Entwicklungskonzept der Deutschen Bundespost}

\subsubsection{ISDN-Grundlagen}

Das ISDN ${ }^{84}$ stellt das Entwicklungsziel der Deutschen Bundespost für die zukunft dar. ISDN steht weltweit für ein universelles, diensteintegrierendes, digitales Fernmeldenetz, das sich aus dem digitalen Fernmeldenetz entwickelt und alle bisherigen und zukünftigen Dienste der Deutschen Bundespost integriert. Zusätzlich bedingt ISDN eine digitale Teilnehmeranschlußleitung, bis hin zum Endgerät, was der sogenannten 'Kommunikations-Steckdose' entspricht. Dieser Teilnehmeranschluß bietet die Option, bis zu acht Endgeräte mit einer einheitlichen Rufnummer von einem Basisanschluß aus $z u$ versorgen und je zwei verschiedene Dienste simultan - auch zu unterschiedlichen zielen - zu betreiben. 85

Die eminenten Verbesserungen der Nutzungsmöglichkeiten telekommunikativer Techniken resultieren einerseits aus der reinen Prozeßinnovation durch die Digitalisierung ${ }^{86}$ und andererseits aus den Produktinnovationen, die durch die Synergieeffekte der Diensteintegration motiviert werden.

Die Produktinnovationen im Bereich der Bewegtbildkommunikation setzen Übertragungskapazitäten in der Größenordnung von $100 \mathrm{Millionen} \mathrm{bit/s} \mathrm{voraus.} \mathrm{Dies} \mathrm{bedingt} \mathrm{den} \mathrm{Einsatz}$ von speziellen Übertragungsmedien. Je nach Medium werden Schmalband- und Breitband-ISDN unterschieden. 87

84 Integrated services Digital Network

$85 \mathrm{Vgl}$. Martin, H.: Die technische Entwicklung zu ISDN, in: Telematikmagazin $\mathrm{Nr} .1$, 7. Jahrgang, 1987, S. $30 \mathrm{ff}$.

86 Durch die Digitalisierung werden größere Übertragungskapazitäten und -qualitäten ermöglicht.

87 Schmalband-ISDN ermöglicht eine Übertragung bis zu $64 \mathrm{kbit} / \mathrm{s}$ und Breitband-ISDN bis $\mathrm{zu} \mathrm{n} \times 64 \mathrm{kbit} / \mathrm{s}$. 
Abbildung 1.5 stellt die Ausbaustufen vom analogen Telefonnetz bis zum integrierenden, breitbandigen Fernmeldenetz dar. Die Anschaltung der ersten kommerziellen ISDN-Anschlüsse fand, mit etwa zweijähriger verspätung, um die Jahreswende $1988 / 89$ statt. ${ }^{88}$ Bis Ende 1990 will die Deutsche Bundespost in mehr als 300 städten ISDN-Anschlüsse ermöglichen. 89 Die Angabe eines detaillierten Einführungszeitpunktes für das Integrierende Breiband-Fernmeldenetz ist momentan nicht möglich.

Abbildung 1.5: Ausbauplanung der Deutschen Bundespost 90

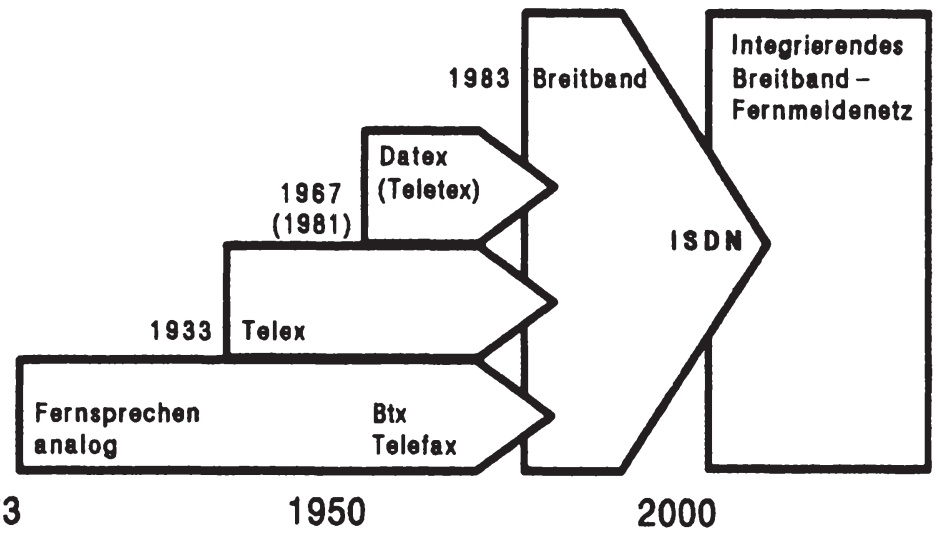

88 Vgl. Cornu, J.: Die Zeit der Zweifel ist vorbei, Strategien für den Durchbruch von ISDN, in: Frankfurter Allgemeine Zeitung $\mathrm{Nr} .67$ vom 20. März 1990, S. B2.

89 Vgl. O.V.: Post will bis Ende 1990 in 300 städten ISDNAnschluß bieten, in: Frankfurter Allgemeine Zeitung $\mathrm{Nr}$. 195 vom 24. August 1989, S. 5.

90 Quelle: Deutsche Bundespost: MEDKOM ein Anwendungsprojekt zur Erprobung der medizinischen Kommunikation im Vorläufer-Breitband-Netz VBN, a.a.O.. 


\subsubsection{I8DN-Ausbaustrategie}

Trotz der hohen Investitionen in den ISDN-Ausbau, können 1993 erst an etwa $10 \%$ der ortsvermittlungsstellen ISDNTeilnehmer unmittelbar angeschlossen werden. Um trotzdem bis 1993 möglichst viele Gebiete versorgen zu können und ab 1993 die angestrebte Flächendeckung $\mathrm{zu}$ erreichen, sieht die Deutsche Bundespost, neben dem normalen Regelausbau, die Möglichkeit der Fremdanschaltung von ISDN-Teilnehmern an eine andere, bereits ISDN-fähige Vermittlungsstelle vor.

\section{Regelausbau}

Im Regelausbau der Deutschen Bundespost werden, wie o.a. zwei Arten von ISDN unterschieden: das Schmalband- und das Breitband-ISDN. Das Schmalband-ISDN wird auf der Basis der vorhandenen Kupfer-Doppelader-Kabel des Telefonnetzes aufgebaut. Es wird mit zwei $64 \mathrm{kbit} / \mathrm{s}$ Nutzkanälen und einem 16 kbit/s steuerkanal 'beaufschlagt'. Das Breitband-ISDN kann sowohl über Glasfaser-Leitungen, als auch über Koaxialkabel 91 realisiert werden, die im Gegensatz zum SchmalbandISDN jedoch vollständig neu aufgebaut werden müssen. Dies geschieht gegenwärtig in Form des Vorläufer-Breitband-Netzes. 92

Basis des ISDN ist, neben der Digitalisierung des Fernsprechnetzes, die Einführung von Netzabschlüssen und einem neuartigen Zeichenabgabesystem zwischen den rechnergesteuerten Vermittlungsstellen. 93 Aufgrund der Unmöglichkeit einer sofortigen Umstellung auf ISDN, hat die Deutsche Bundespost grundsätzlich entschieden, den Ausbau 'nachfrageorientiert' $\mathrm{zu}$ betreiben. Die Einführung des ISDN soll, von

91 Mit reduzierter Übertragungskapazität.

92 Vgl. Schön, H.: ISDN und ökonomie, in: Schwarz-Schilling, C., Florian, W. (Hrsg.): Jahrbuch der Deutschen Bundespost 1986, Bad Windsheim 1986, S. 10.

$93 \mathrm{Vgl}$. ebenda S. $30 \mathrm{ff}$. 
den wirtschaftszentren ausgehend, möglichst schnell in die dezentral gelegenen Regionen ausgebreitet werden. 94

Ausgehend von den nachfrageträchtigen Geschäftsgebieten, wird der Ausbau des Vorläufer-Breitband-Netzes vorangetrieben. Für die Übergangszeit, bis zur flächendeckenden Verkabelung mit Glasfaser, wird gebietsweise eine parallele Versorgung durch symmetrische Kupfertechnik erfolgen.

Langfristig soll die letzte Entwicklungsstufe, das 'Integrierte Breitbandfernmeldenetz', auch Universalnetz genannt, erreicht werden. Das Universalnetz erlaubt, neben dem Betreiben von Individualkommunikation, die Massenkommunikation.

\section{Fremdanschaltungen}

Mit Hilfe von 'Fremdanschaltungen', soll eine frühzeitige, 'quasi' flächendeckende, ISDN-Bereitstellung geschaffen werden, um Teilnehmer in noch nicht mit ISDN versorgten Regionen mit Schmalband-ISDN zu erreichen. Bei der Fremdanschaltung werden grundsätzlich zwei Anschlußarten unterschieden: 95

1. Fremdanschaltungen im gleichen Orstnetz. Die Basisanschlüsse werden, mit Hilfe von Konzentratoren und Primärmultiplexanschlüssen, unmittelbar an die ISDN-Vermittlungsstelle herangeführt.

2. Fremdanschaltungen im Fernnetz. Wenn im 'eigenen' ortsnetz noch keine ISDN-fähige Vermittlungsstelle vorhan-

94 Vgl. Minister für Wirtschaft Mittelstand und Technologie des Landes Nordrhein-Westfalen und Deutsche Bundespost (Hrsg.): Telekommunikations-Atlas Nordrhein-Westfalen Band 2, a.a.0., S. 71 .

$95 \mathrm{Vgl.} \mathrm{Bundesminister} \mathrm{für} \mathrm{das} \mathrm{Post-und} \mathrm{Fernmeldewesen:}$ ISDN - die Antwort der Deutschen Bundespost auf die Anforderungen der Telekommunikation von morgen, Bonn 1984 , S. 19. 
den ist, erfolgt der Anschluß an eine ISDN-fähige ortsvermittlungsstelle im ortsnetz der zuständigen Knotenvermittlungsstelle. 96

Für den Zeitraum vor 1991 bietet, die Deutsche Bundespost eine ISDN-Sonderanschaltung für den Fall an, daß aus Entfernungsgründen eine Fremdanschaltung nicht eingesetzt werden kann. Für den Teilnehmer wird die Sonderanschaltung jedoch mit erheblichen Kosten verbunden sein, was einer Nutzung entgegenstehen könnte.

ISDN-Fernanschlüsse sind eine kostenintensive Lösung zur Erreichung einer ISDN-Flächendeckung. Die Voraussetzungen, welche die Deutsche Bundespost für den Einsatz dieser provisorischen Anschlußmöglichkeiten zu Grunde legt, besonders unter dem Aspekt von eventuellen Mindestteilnehmerzahlen in den betroffenen Regionen, sind bisher nicht geklärt. Hier können, durch zu lange Wartezeiten oder Mindestteilnehmerzahlen, regionale Disparitäten entstehen, die das vorhandene Nord-Süd-Gefälle weiter verstärken. Dies gilt im besonderen für den Fall, daß weitere Verzögerungen im ISDN-Ausbau die ISDN-Anschlußmöglichkeit um mehrere Jahre verzögert.

\subsubsection{I8DN-Tarife}

Die neue Telekommunikationsordnung, in der die Tarife für das ISDN enthalten sind, ist am 1. Januar 1988 in Kraft getreten. Im ISDN werden zwei Universalanschlüsse, mit den Anschlußarten Basisanschluß und Primärmultiplexanschluß 97 , angeboten. Die Universalanschlüsse können innerhalb folgen-

96 Die Fremdanschaltung im Fernnetz ist ab 1991 geplant.

97 Der Primärmultiplexanschluß bietet 30 Basiskanäle mit jeweils $64 \mathrm{kbit} / \mathrm{s}$ und wird nur für Anschaltungen von mittleren und großen Kommunikationsanlagen überlassen. 
der Telekommunikationsdienste genutzt werden:

- im Telefon-Dienst,

- im Teletex-Dienst,

- im Telefax-Dienst,

- im Bildschirmtext-Dienst,

- im Datenübermittlungs-Dienst und

- im Funkruf-Dienst.

Für die betriebsfähige Bereitstellung von Universalanschlüssen, werden je Anschluß folgende Gebühren erhoben:

Tabelle 1.3: Grundgebühr für den ISDN-Anschluß 98

\begin{tabular}{|l|c|c|}
\hline & $\begin{array}{c}\text { Bereitstellungsgebühr } \\
\text { einmalig }\end{array}$ & $\begin{array}{c}\text { Grundgebüh in DM } \\
\text { monatlich }\end{array}$ \\
\hline \hline $\begin{array}{l}\text { Basisanschluß } \\
(2 \times 64 \mathrm{kbit} / \mathrm{s})\end{array}$ & $130,--$ & $74,--$ \\
\hline $\begin{array}{l}\text { Primärmultiplex- } \\
\text { anschluß } \\
(30 \times 64 \mathrm{kbit} / \mathrm{s})\end{array}$ & $200,--$ & $518,--$ \\
\hline
\end{tabular}

Die Deutsche Bundespost ist beim ISDN davon abgegangen, die monatliche Grundgebühr in Abhängigkeit der genutzten Dienste zu berechnen.

Die Verbindungsgebühren für den Aufbau einer B-Kanalverbindung im ISDN, entsprechen den Verbindungsgebühren im analogen Telefonnetz. Die Gebühren für die Wählverbindungen im ISDN sind somit dienstunabhängig, jedoch zeit- und zonenabhängig.

Fazit: 1. Die Übertragung großer Text- und Datenmengen wird

$98 \mathrm{Vgl}$. Deutsche Bundespost: Die wichtigsten Gebühren, 0.0., Stand September 1988. 
im ISDN wesentlich billiger als bei den heutigen Spezialdatennetzen.

2. Kurzdialoge im Bereich der Text- und Datenübertragung werden, aufgrund der Bereitstellungsgebühr von 0,23 DM pro Verbindung, im ISDN teurer als bei den heutigen Text- und Datennetzen. 99

\subsection{Effekte der Telematik}

\subsubsection{Gesamtwirtschaftliche Effekte der Telematik}

Bei der Diskussion der Effekte der Telematiknutzung sind die vermuteten gesamtwirtschaftlichen wirkungen von eminenter Bedeutung. Von primärem Interesse sind hierbei die Einflüsse auf das gesamtwirtschaftliche Wachstum, die Produktivitätsentwicklung und die Beschäftigung. Exakte Aussagen zu dieser Thematik bedingen jedoch eine mikroökonomisch fundierte Totalanalyse der Anpassungsprozesse des gesamten Wirtschafts - und Gesellschaftssystems, was, vom gegenwärtigen Erkenntnisstand ausgehend, nicht möglich ist. 100 Tendenzaussagen und Partialbetrachtungen sollten jedoch möglich sein und werden im folgenden dargelegt.

99 Vgl. Minister für Wirtschaft Mittelstand und Technologie des Landes Nordrhein-Westfalen und Deutsche Bundespost (Hrsg.): Telekommunikations-Atlas Nordrhein-Westfalen Band 2, a.a.0., S. 89 .

100 Vgl. Neumann, K.; Schnöring, T.: Das ISDN - Ein Problemfeld aus volkswirtschaftlicher und gesellschaftspolitischer Sicht, in: Jahrbuch der Deutschen Bundespost, a.a.0., s. 56 . 


\subsubsection{Generelle Aspekte}

Die Produktion, Verarbeitung und Verteilung von Informationen stellt in modernen Industriegesellschaften einen Produktionsfaktor von dominierender Bedeutung dar. ${ }^{101}$ Die Notwendigkeit zum Informationsaustausch wird durch die zunehmende gesamtwirtschaftliche Arbeitsteilung erhöht. Der effizienten Gewinnung, Übermittlung und Verarbeitung von Information kommt eine fundamentale Bedeutung $\mathrm{zu}$, wobei der Ausbaustand der Kommunikationsnetze ein wichtiger Bestandteil der gesamtwirtschaftlichen Infrastruktur ist. Aufgrund der, mit der Anwendung der Telematik erwarteten, verbesserten Allokation der volkswirtschaftlichen Ressourcen, wird mit einer Verstärkung der Wettbewerbsfähigkeit der Wirtschaft der Bundesrepublik Deutschland auf den Weltmärkten gerechnet. 102

Neben den, in der Informationsbeschaffung, -übermittlung und -verarbeitung, generell erwarteten Effizienzsteigerungen, ermöglicht die erhöhte Disponibilität der Informationen, bei gleichzeitiger senkung der Informationskosten, eine verbesserte Markttransparenz.

Ausgehend von der starken Exportabhängigkeit der bundesdeutschen wirtschaft, kommt ihrer internationalen Wettbewerbsfähigkeit eine zentrale Bedeutung zu. Da die Konkurrenzfähigkeit durch die erwarteten Kostenvorteile gestärkt werden kann, ist der im Vergleich zu den Hauptkonkurrenzländern zügige Ausbau der neuen Telekommunikationstechniken von außerordentlicher Bedeutung.

$101 \mathrm{Vgl}$. Enquete-Kommission: Neue Informations- und Kommunikationstechniken, Kommission des Deutschen Bundestages, Drucksache 9/2442, Bonn 1983, S. 77 .

$102 \mathrm{Vgl}$. ebenda S. 78. 


\subsubsection{Beschäftigungseffekte}

Die Aussagen über Auswirkungen der neuen Telekommunikationstechniken auf die Beschäftigung sind heterogen und bilden ein breites Meinungssprektrum. Die Diskussion konzentriert sich grundsätzlich auf zwei Problembereiche:

- die Auswirkungen der Telekommunikation auf 'quantitative Beschäftigungseffekte' und

- die Auswirkungen der Telekommunikation auf 'qualitative Beschäftigungseffekte'.

Der Diskussionsstand wird im folgenden dargestellt. 103

\section{Effekte auf das qualitative Beschäftigungsniveau}

Die Effekte der Telematik auf das Beschäftigungsniveau sind in der politischen und ökonomischen Diskussion umstritten. Meinungen, daß deutliche Personalreduzierungen primär im Bürobereich stattfinden, stehen Aussagen gegenüber, daß sich der Personalabbau und die zusätzlich induzierte Personalnachfrage zumindest die Waage halten. Unter ökonomischen Aspekten wird argumentiert, daß kurzfristige negative Beschäftigungseffekte in Kauf genommen werden müssen, um langfristig eine Arbeitsplatzsicherung $\mathrm{zu}$ erreichen. 104

Grundsätzlich lassen sich die Beschäftigungseffekte in folgende drei Bereiche untergliedern: 105

103 Vgl. Hessischer Minister für Wirtschaft und Technik: Nutzung der Informations- und Kommunikationstechnik in Hessen, in: Beiträge zur Wirtschaftspolitik in Hessen, Heft 13, Wiesbaden 1987 , S. 36 .

$104 \mathrm{Vgl}$. Winkelhage, F.: Wirkungen neuer Medien auf Arbeitsteilung und Arbeitsabläufe in Wirtschaft und Verwaltung, in: Bundesforschungsanstalt für Landeskunde und Raumordnung (Hrsg.): Informationen zur Raumentwicklung, Heft 3, 1982, S. 206.

$105 \mathrm{Vgl.}$ Ewers, H.; Fritsch, M.: Telematik und Raumordnung, a.a.0., s. 37 . 
- die direkten Beschäftigungswirkungen bei den Anwendern,

- die expansiven Beschäftigungseffekte bei den Produzenten entsprechender Hard- und Software 106 und

- die Beschäftigungszunahmen aufgrund der gesteigerten Wettbewerbsfähigkeit und der, im Zusammenhang zur Telematik stehenden, produktinnovationen.

Von den direkten Beschäftigungseffekten der Telematik am stärksten betroffen sind die Aktivitätsbereiche, in denen vorwiegend Informationen verarbeitet, produziert oder übermittelt werden. Bisherige Untersuchungen wurden deshalb primär auf die sogenannten 'Informationsbeschäftigten' bezogen, die in den studien jedoch unterschiedlich klassifiziert wurden, was $\mathrm{zu}$ divergierenden Ergebnissen führte. 107

Die Studien der Enquete-Kommission ${ }^{108}$ und von Fischer 109 unterscheiden die Informationsbeschäftigten nach der Art der Betroffenheit. Beschäftigten, die primär Routinetätigkeiten ausüben, werden Rationalisierungen und eine entsprechende Abnahme der Beschäftigungsmöglichkeiten prognostiziert. Im Gegensatz dazu, stehen die nicht routinierbaren 'egghead-' Tätigkeiten, die relativ hohe Qualifikationsanforderungen an den Ausübenden stellen. ${ }^{110}$ Die Enquete-Kommission prognostiziert für den Büro- und Verwaltungsbereich einen Beschäftigungsabbau von 25\%, was ca. 2 bis 2,5 Mil-

106 Auf eine nähere Darstellung der Beschäftigungswirkungen bei den Produzenten wird im folgenden verzichtet.

$107 \mathrm{Vgl}$. Ewers, H.; Fritsch, M.: Telematik und Raumordnung, a.a.0., S. 39 .

108 Enquete-Kommission: Neue Informations- und Kommunikationstechniken, a.a.o..

109 Fischer, K.: Telekommunikation, Raumordnung und regionale Strukturpolitik, Köln 1984.

$110 \mathrm{Vgl.}$ Ewers, H.; Fritsch, M.: Telematik und Raumordnung, a.a.o., S. 42 . 
lionen Arbeitsplätzen entspricht. 111 Das definitive Ausmaß wird jedoch u.a. davon abhängen, inwieweit Produktivitätssteigerungen zur Verbesserung des Dienstleistungsangebotes beitragen werden.

Expansive Beschäftigungseffekte werden nicht nur aufgrund verbesserter Wettbewerbsbedingungen durch Kostenreduzierungen motiviert, sondern gehen auch von Produktinnovationen aus, die der Einsatz neuer telekommunikativer Techniken ermöglicht. Diese expansiven Beschäftigungseffekte sind jedoch quantitativ kaum erfaßbar. Die Wirkungen, die auf indirekten Effekten beruhen, sind nahezu nicht prognostizierbar, da sie sich erst langfristig auswirken. 112

Nach der im Auftrag des Bundesministers für Forschung und Technologie durchgeführten Meta-studie bedingt eine erhöhte Innovationsaktivität lediglich unter günstigen Bedingungen eine höhere Beschäftigung als nach der status-quo-Entwicklung. 113 "Aber selbst bei pessimistischen Annahmen erweisen sich beschäftigungspolitisch erhöhte Innovationsanstrengungen vorteilhafter als ein Innovationsverzicht". 114

Insgesamt gesehen, kann zum gegenwärtigen zeitpunkt keine abschließende Aussage über die Beschäftigungseffekte der Telematik auf die Anwender getroffen werden. Ursache hierfür sind die mangelnde Erfaßbarkeit der gesamtwirtschaftlichen Kompensationseffekte und deren temporär differenziertes Aufkommen. Die Beschäftigungseffekte vollziehen sich bis

$111 \mathrm{Vgl}$. Enquete-Kommission: Neue Informations- und Kommunikationstechniken, a.a.0., s. 104 .

112 Vgl. Brepohl, K.: Telematik - Die Grundlage der Zukunft, Bergisch Gladbach 1982 und Ewers, H.; Fritsch, M.: Telematik und Raumordnung, a.a.0., s. $38 \mathrm{f}$.

113 Vgl. Matzner, E. U.a.: Beschäftigungsrisiko Innovation? - Arbeitsmarktwirkungen moderner Technologien; Befunde aus der Meta-studie, Berlin 1988, S. 121.

114 Ebenda. 
heute jedoch in der Regel erheblich langsamer als vielfach prognostiziert wird. 115

\section{Effekte auf die Qualifikation}

Der Einsatz neuer telekommunikativer Techniken stellt nicht nur eine Substitution der alten Kommunikationstechnik durch neue dar, sondern impliziert Veränderungen im Bereich der Arbeitsinhalte, der Arbeitsorganisation und der Qualifikationsanforderungen der Beschäftigten, die mit der Beschaffung, Verknüpfung und weitergabe von Informationen in $\mathrm{Zu}-$ sammenhang stehen. 116

Grundsätzlich kann davon ausgegangen werden, daß, durch den Einsatz der neuen Telekommunikationstechniken, sich die Qualifikationen der Beschäftigten ändern. Neue Tätigkeiten werden entstehen und bekannte Tätigkeiten auf andere Art und Weise ausgeführt. Neben den erwarteten positiven Effekten wird die Veränderung der Tätigkeitsinhalte auch negative Effekte implizieren. 117

Die erwarteten positiven Beschäftigungseffekte liegen in einer Höherqualifizierung, Kompetenzerweiterung und Zunahme der Autonomie, was primär auf folgende Punkte zurückzuführen ist: 118

115 Vgl. Schnöring, T.: Wirkungen der Telekommunikationsentwicklung auf Anwender, in: Deutsche Telepost Consulting GmbH (Hrsg.): Aspekte der Telekommunikationsentwicklung im Land Rheinland-Pfalz, Bonn, Mainz 1987, S. 34 .

$116 \mathrm{Vgl}$. Bodendorf, F.; Lüthi, A.: Neue Informations- und Kommunikationstechnologien, in: Die Unternehmung $\mathrm{Nr} .5$, 43. Jahrgang 1989, S. $347 \mathrm{ff}$.

$117 \mathrm{Vgl}$. Ewers, H.: Räumliche Innovationsdisparitäten und räumliche Diffusion neuer Technologien, in: Brugger, E. (Hrsg.): Regionale Innovationsprozesse und Innovationspolitik, Diessenhofen 1984, S. 104 .

$118 \mathrm{Vgl}$. Enquete-Kommission: Neue Informations- und Kommunikationstechniken, a.a.0., S. $105 \mathrm{ff}$. 
- Der Anteil an komplexen Tätigkeiten wird steigen, und die Routinetätigkeiten werden reduziert.

- Bisher getrennte Tätigkeiten werden zusammengelegt und neue Tätigkeitsbereiche gebildet.

- Informationsbezogene Tätigkeiten werden eingegliedert und zugang zu mehr Information gewährt.

Die erwarteten negativen Effekte liegen im Bereich einer Dequalifizierung, Kompetenzbeschränkung und Verringerung der Autonomie, begründet durch:

- die Reduzierung von Tätigkeiten auf einfache Prozeduren, deren Automatisierung unwirtschaftlich ist und

- die Zerlegung und Monotonisierung von Arbeitsabläufen. 119

Als Folge der angeführten Veränderungen, wird eine Polarisierung der Qualifikationsstrukturen erwartet. ${ }^{120}$ Ausdruck dieser Polarisierung ist der Abbau der mittleren Qualifikationsebene, bei gleichzeitiger Konzentration der Beschäftigten auf hohen und niedrigen Qualifikationsebenen. 121 während niedrig Qualifizierte mit weiteren Einschränkungen ihrer Tätigkeitsentwicklung rechnen müssten, werden höher Qualifizierte von Routinearbeiten weiter entlastet und mit zusätzlichen anspruchsvollen Arbeiten beauftragt. 122

Die Beschäftigungsprognosen der Prognos AG und des Institutes für Arbeitsmarkt- und Berufsforschung von 1985 kommen zu dem Ergebnis, daß bis zum Jahr 2000 ein Großteil der Be-

119 Vgl. ebenda, S. 105; Friedrich, J. U.a.: Organisationsmodelle für das Büro von morgen, in: office Management $3 / I-1987$, S. 22 .

$120 \mathrm{Vgl}$. Hessischer Minister für Wirtschaft und Technik: Nutzung der Informations- und Kommunikationstechnik in Hessen, a.a.0., s. 37 .

$121 \mathrm{Vgl.}$ Funck, R.: Technologiepolitik und räumliche Struktur, in: Böventer von, E. (Hrsg.): Regionale Beschäftigung und Technologieentwicklung, Berlin 1989, S. 120.

$122 \mathrm{Vgl}$. ebenda S. $37 \mathrm{f}$. 
schäftigten (80\%) vom technischen wandel der Nachrichtenund Kommunikationstechniken positiv oder negativ betroffen sein werden. Da sich die positiv und negativ betroffenen Bereiche in etwa die waage halten, werden lediglich minimale Auswirkungen auf die Beschäftigung insgesamt erwartet. 123

Die Arbeitswelt von morgen wird zunehmend die Fähigkeit der Zusammenarbeit und Kommunikation zwischen Mensch und Maschine erfordern. Die endgültige Ausgestaltung der zukünftigen Entwicklung ist, nach Meinung der Enquete-Kommission, jedoch abhängig von der konkreten Arbeitsplatzgestaltung. Dies impliziert ein potentielles steuerungspotential für die zukünftige Entwicklung. 124

\subsubsection{Regionalpolitische Effekte der Telematik}

\subsubsection{Gegenwärtige Entwicklungstendenzen}

In der Bundesrepublik Deutschland lassen sich gegenwärtig drei Trends der Regionalentwicklung beobachten: 125

\section{Kleinräumliche Dezentralisierung}

Innerhalb der Agglomerationsräume findet eine zunehmende Suburbanisierung statt. Auf Kosten der Kernstädte verstärkt sich die 'kleinräumliche Dezentralisierung' von Einwohnern und Arbeitsstätten. Von diesem Trend sind alle Großstädte

123 Vgl. Schnöring, T.: Wirkungen der Telekommunikationsentwicklung auf Anwender, a.a.0., S. 25.

$124 \mathrm{Vgl}$. Enquete-Kommission: Neue Informations- und Kommunikationstechniken, a.a.0., s. 78 ff.

$125 \mathrm{Vgl}$. Sandvoß, J.: Fernarbeit: Formen und Potentiale dezentralisierter Arbeit im Lichte qualifikations- und regionalpolitischer Aspekte, Frankfurt u.a. 1989, S. 234 . 
und größeren städte in den hochentwickelten Regionen betroffen, lediglich Ausmaß und Geschwindigkeit der Entwicklung sind unterschiedlich. 126

\section{Großräumige Konzentration in den Agglomerationsräumen}

Parallel zur fortschreitenden Konzentration in den Agglomerationen, von der primär die dispositiven und tertiären Informationsaktivitäten betroffen sind, werden operative Funktionen aus den Innenstädten in die Agglomerationsränder bzw. ländlich-peripheren Gebiete verlagert. Leitungs- und Kontrollfunktionen verbleiben in den zentren oder werden dorthin versetzt. 127 Auflösungserscheinungen von strukturstarken Verdichtungsräumen sind nicht zu beobachteten.

\section{Nord-süd-Gefälle zwischen den Agglomerationen}

Die Disurbanisation der altindustriellen, strukturschwachen Verdichtungsräume $\mathrm{zu}$ Gunsten der 'High-Tech'-Agglomerationen wird als räumlicher Ausdruck der strukturverschiebungen innerhalb der Volkswirtschaft angesehen. Die auf weitgehend fehlenden altindustriellen Belastungen und Konzentration auf neue Technologien sowie Forschungs- und Entwicklungsaktivitäten basierende positive Entwicklung im süden, führt $z u$ weiteren Ansiedlungen zukunftsträchtiger Branchen in diesen Regionen. 128 Ein zusätzlicher Faktor des Nord-südGefälles ist das 'Image' einer Region. Standortfaktoren wie Ambiente, Freizeitgestaltungsmöglichkeiten und attraktive Wohn- und Arbeitsumgebung beeinflussen zunehmend die stand-

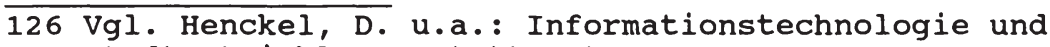
stadtentwicklung, stuttgart 1984, s. 65.

127 Vgl. Bade, F.: Räumliche Anpassungsprozesse, Arbeitsteilung und unternehmerisches standortverhalten, in: Brugger, E. (Hrsg.): Regionale Innovationsprozesse und Innovationspolitik, a.a.o., S. $119 \mathrm{ff}$.

$128 \mathrm{Vgl}$. Julitz, L.: Bayern, Baden-Württemberg und Hessen liegen vorn, in: Frankfurter Allgemeine Zeitung Nr. 254 vom 31. Oktober 1988, S. 18 und Henckel, D. u.a.: Informationstechnologie und stadtentwicklung, a.a.o. S. 167 . 
ortentscheidung. 129

Das regionalpolitische Problem des Nord-Süd-Gefälles existiert auch in Hessen. Strukturverschiebungen der Arbeitsplätze und Einwohner implizieren erhebliche Probleme für strukturschwache Regionen. Die Erkenntnis, "tomorrow's industries are not going in yesterday's regions"130, weckt regionalpolitischen Handlungsbedarf, der diesem Trend entgegenwirkt.

Die als 'Süddrift der Wirtschaftskraft und des Wohlstandes' bezeichnete Entwicklung ist zwar ursächlich nicht von den neuen Telekommunikationstechniken ausgelöst worden, kann jedoch durch die Telematik verstärkt oder abgeschwächt werden.

\subsubsection{Wirkungsthesen der Telematik}

Grundsätzlich wirken telekommunikative Techniken gleichartig. 131 Ihr Einsatz bewirkt per se keine Unterschiede, da in gleichartigen Unternehmen im Prinzip gleichartige Effekte auftreten. Aufgrund der unterschiedlichen regionalen Branchenstruktur und dem unterschiedlich schnellen Ausbau der telekommunikativen Infrastruktur, sind räumlich unterschiedliche wirkungen jedoch zwangsläufig.

129 Vgl. Reese, J.: Die Bedeutung der neuen Fernmeldedienste für Nordhessen, Gutachten im Auftrag der Hessischen Staatskanzlei, Kassel 1986, S. 46 ff.

130 Hall, P.: The Geography of the Fifth Kondratieff Cycle, in: New Society, Band 55, 1981, S. 537.

$131 \mathrm{Vgl}$. Henckel, D. u.a.: Informationstechnologie und Stadtentwicklung, a.a.O., S. 64 ; Sandvoß, J.: Fernarbeit: Formen und Potentiale dezentralisierter Arbeit im Lichte qualifikations- und regionalpolitischer Aspekte, a.a.0., S. 236 . 
Über die Art der räumlichen wirkungen neuer telekommunikativer Techniken besteht in der Literatur kein Konsens. In jüngeren Veröffentlichungen kristallisieren sich verschiedene Thesen über mögliche räumliche Wirkungen der Telematik heraus, die in den folgenden Begriffspaaren und anhand der These von den eingeschränkten Innovationswirkungen dargestellt werden: 132

$\begin{array}{rll}\text { Dekonzentration } & -- & \text { Konzentration } \\ \text { Dezentralisierung } & -- & \text { Zentralisierung } \\ \text { Nivellierung } & -- & \text { Polarisierung } \\ \text { Trendsetter } & -- & \text { Trendverstärker }\end{array}$

Darüberhinaus hat sich die Diskussion in der Literatur in Richtung einer Ambivalenz der wirkungen entwickelt, welche in Kapitel 1.3.2.3. dargestellt werden.

Die Thesenpaare Dekonzentration -- Konzentration und Dezentralisierung -- Zentralisierung werden aufgrund der erwarteten gegenläufigen Entwicklung begrifflich nicht, wie in der Literatur stellenweise anzutreffen, synonym verwendet. Dekonzentration und Konzentration betreffen die räumliche Verteilung von Arbeitsstätten und Wohnungen, Dezentralisierung und Zentralisierung die räumliche Verteilung von Steuerungskompetenz und Entscheidungsmacht.

\section{Dekonzentration -- Ronzentration}

Die Vertreter der Dekonzentrationsthese 133 gehen nach relativ kurzer zeit von einer ubiquitären Bereitstellung der neuen Telekommunikationsdienste aus. Sie argumentieren, daß traditionelle Standortvorteile der Agglomerationen abgebaut würden und eine gleichmäßige Verteilung von Arbeitsstätten

132 Vgl. Spehl, H.: Räumliche wirkungen der Telematik, a.a.0., S. $258 \mathrm{ff}$.

$133 \mathrm{Vgl}$. exemplarisch Fischer, K.: Telekommunikation, Raumordnung und regionale strukturpolitik, a.a.o.; storbeck, D.: Telekommunikation und siedlungsstruktur, in: Arch $+\mathrm{Nr}$. 75/76, 1984, S. 53-55. 
und Wohnungen im Raum zu einer räumlichen Dekonzentration führt. 134

Erwartete Dekonzentrationen ergeben sich beispielsweise durch: 135

- die Möglichkeit einzelne Betriebsteile auf verschiedene Standorte $\mathrm{zu}$ verteilen,

- die Teleheimarbeit sowie

- die verstärkte Nutzung von Homebanking und Teleshopping.

Die Vertreter der Konzentrationsthese 136 gehen von einer Fortdauer der agglomerativen Tendenzen aus. Unter dem Aspekt einer temporär differenzierten Diffusion der Telematik, die primär in den Agglomerationen beginnt, wird zunächst eine weitere großräumige Konzentration in den Verdichtungsräumen erwartet, einhergehend mit fortschreitenden Abwanderungen aus den ländlich-peripheren Bereichen .137

Die räumliche Konzentration wird primär auf kurz- bis mittelfristiger Ebene erwartet. Dekonzentrationstendenzen werden langfristig - auch von den Vertretern der Konzentrationsthese - einhergehend mit der Ubiquität der neuen Telekommunikationstechniken prognostiziert.

134 Vgl. Spehl, H.: Räumliche Wirkungen der Telematik, a.a.O., S. 258 .

$135 \mathrm{Vgl}$. Bördlein, R.: Informationstechnisch bedingte raumstrukturelle Veränderungen im Frankfurter Umland, untersucht am Beispiel der Anbieter neuer Informationsund Kommunikationstechnologien, Frankfurt 1986, S. 22.

$136 \mathrm{Vgl}$. exemplarisch Hoberg, R.: Zur Analyse von räumlichen Diffusionsprozessen bei der Telematikanwendung, in: Akademie für Raumforschung und Landesplanung (Hrsg.): Räumliche Wirkungen der Telematik, a.a.o, s. 73 ff; Küpper, U.: Neue Technologien, Wirtschaftswachstum und Innenstadtentwicklung, in: Archiv für Kommunalwissenschaften 24,1985, S. $189 \mathrm{ff}$.

137 Vgl. Spehl, H.: Räumliche wirkungen der Telematik, a.a.o., S. 259 . 


\section{Dezentralisierung -- zentralisierung}

Die Zentralisierung führt zu einem Anwachsen der Macht in den Unternehmenszentralen, was eine verstärkte Abhängigkeit der Peripherie von den Zentren zur Folge hat. ${ }^{138}$ Neben der erwarteten Disurbanisisierung, "besteht nahezu einhellige Übereinstimmung, daß die Entwicklung der Telematik die Zusammenfassung von Kontroll- und Entscheidungsbefugnissen in den Zentren tendenziell begünstigt. Es ist sogar durchaus wahrscheinlich, daß eine ungesteuerte Entwicklung der Telematik zu einer zentralisierung bei gleichzeitiger Dekonzentration führt." 139

Unter Dezentralisierung wird die Verteilung von bisher zentral ausgefühten steuerungsfunktione auf mehrere kleinere Einheiten verstanden. Einzelne Unternehmensbereiche könnten größere Entscheidungskompetenzen erhalten und Koordinationsaufgaben auf die Zentrale beschränkt werden. 140

Der Dezentrallisierungseffekt konnte bereits von Klatzky im Bereich der klassischen EDV nachgewiesen werden, 141 wobei der folgende, als Kaskadeneffekt bezeichnete, Wirkungszusammenhang beobachtet wurde: Die Utbernahme von Routineentscheidungen der untersten Hierarchiestufen durch den Computer, erlaubt den unmittelbaren Vorgesetzten einfache Ent$138 \mathrm{Vgl}$. ebenda, S. 260 .

139 Spehl, H.: Räumliche wirkungen der Telematik, in: Bundesforschungsanstalt für Landeskunde und Raumordnung (Hrsg.): Räumliche Auswirkungen der neuen Informationsund Kommunikationstechniken, Bonn, Hannover 1985, S. 260 .

$140 \mathrm{Vgl}$. Fischer, K.: Telekommunikation, Raumordnung und regionale Strukturpolitik, a.a.0., S. 129; Sydow, J.: organisationsspielraum und Büroautomation, Berlin/New York 1985, S. 107 f.; Weltz, F.: Veränderung der Leitungsfunktion durch neue Technik, in: Office Management 5/1985, S. 108 .

$141 \mathrm{Vgl}$. Klatzky, S.: Automation, Betriebsgröße und die Verteilung von Entscheidungskompetenzen - Der Kaskadeneffekt, in: Grochla, E. (Hrsg.): Organisationstheorie, 2. Bd., stuttgart 1976, S. $518 \mathrm{ff}$. 
scheidungen nach unten zu delegieren; dieser Vorgang wiederholt sich bis zur organisationsspitze. 142

Zusammenfassend läßt sich feststellen, daß momentan keine eindeutige, technologieinduzierte Tendenz aufgezeigt werden kann und somit auch in Bezug auf den Dezentralisierungsgrad keine eindeutige Entwicklung erkennbar ist.

\section{Nivellierung -- Polarisierung}

Ausgehend von der, unter status-quo-Bedingungen, erwarteten großräumigen Zentralisierung und Konzentration bei kleinräumiger Dekonzentration, wird von verschieden Autoren ein Einfluß der Telematik auf das Verhältnis der Agglomerationen zueinander vermutet.

Unter Polarisierung wird die Herausbildung einer Hierarchie der bestehenden zentren verstanden, die momentan durch ein Nord-Süd-Gefälle, zwischen den ungünstiger entwickelten Gebieten im Norden und den informationstechnisch orientierten Zentren im Süden, geprägt ist. Die Entwicklung und Anwendung der Informationstechnologie setzt, aufgrund der Ausgangssituationen der Agglomerationen, einen sich selbst verstärkenden Prozeß in Gang, der die Konzentration im südlicheren Raum begünstigt. 143 Ein Ausgleich wird erst auf lange sicht durch neue Initialregionen erwartet. 144

Die Nivellierungsthese geht davon aus, daß mit der Telematik die Disparitäten zwischen den Agglomerationen reduziert

$142 \mathrm{Vgl.}$ ebenda S. 523; Kieser, A.; Kubicek, H.: Organisation, 2. Aufl., Berlin/New York 1983, S. 312.

$143 \mathrm{Vgl}$. Türke, K.: Die Herausforderung neuer Informationsund Kommunikationstechniken an die Raumplanung: Das elektronische Dorf - Die verkabelte Stadt, in: Akademie für Raumforschung und Landesplanung (Hrsg.): Arbeitsmaterial Nr. 81, Hannover 1984, S. 5.

$144 \mathrm{Vgl}$. Gschwind, F.; Henckel, D.: Innovationszyklen der Industrie - Lebenszyklen der städte, in: Bauwelt 24, stadtbauwelt $82 / 1984$, S. 134 . 
werden könnten oder durch entsprechende Förderung eine positive wirtschaftsentwicklung initiiert wird. Eine Nivellierung wird jedoch in der Literatur, aufgrund der ungünstigen Ausgangssituation der altindustrialisierten Regionen, eher skeptisch beurteilt. 145

\section{Trendsetter -- Trendverstärker}

Die Trendverstärkungsthese geht davon aus, daß die Telematik keine eigene Entwicklung induziert, sondern bereits vorhandene Trends verstärkt. Dies wird besonders für die Einführungszeit der neuen Telekommunikationstechniken erwartet, in der primär die gegebenen Probleme mit der Telematik bewältigt werden.

Ausgehend von den innovatorischen Raumüberwindungstechnologien der Historie, zeigt sich, daß die meisten bedeutenden Einsatzmöglichkeiten erst mit zeitlicher Verzögerung erkannt und genutzt wurden. Unter dieser Perspektive, wird die Frage nach der Möglichkeit eines Trendsetters wahrscheinlich erst geraume zeit nach der endgültigen Einführung der Telematik geklärt werden. 146

\section{These der eingeschränkten Innovationswirkungen}

Nach der These der eingeschränkten Innovationswirkungen, werden die Effekte auf die räumliche Entwicklung wesentlich geringer sein als zumeist angenommen. Die sozialen Prozesse der Diffusion und Anwendung neuer Techniken treten mit erheblichen zeitlichen Verzögerungen gegenüber der rein technischen Innovation auf. Die These konzentriert sich nicht auf bestimmte Richtungen der Effekte, sondern auf

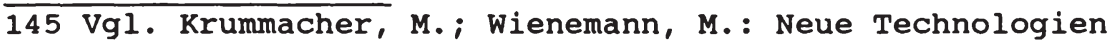
und Hoffnung auf eine Renaissance für das Ruhrgebiet, in: Raumplanung $32 / 1986$, S. $37 \mathrm{ff}$.

$146 \mathrm{Vgl}$. Heinze, G.; Kill, H.: Chancen und Grenzen der neuen Informations- und Kommunikationstechniken - Zur Übertragung verkehrsevolutorischer Erfahrungen auf die Telekommunikation, in: Akademie für Raumforschung und Landesplanung (Hrsg.): Räumliche Wirkungen der Telematik, a.a.o., s. $21 \mathrm{ff}$. 
deren zeitlichen Ablauf. 147

\subsubsection{Ambivalenz räumlicher wirkungen}

In der Diskussion um die räumlichen wirkungen der Telematik hat sich inzwischen die These einer Ambivalenz weitgehend durchgesetzt. 148 sie beinhaltet das Auftreten gegenteiliger räumlicher Wirkungen, die weitgehend von den Handlungen der gesellschaftlichen Akteure determiniert sind. Die Telematik bietet sowohl ein beträchtliches Dezentralisierungs- und Dekonzentrationspotential, als auch eine Tendenz, die, unter Status-quo-Bedingungen und ohne deutliche Eingriffe, eher zu einer Zentralisierung, Konzentration und Polarisierung in der räumlichen Entwicklung führt. 149

Die Prognos AG sieht die Entwicklung der räumlichen Wirkungen im Sinne einer Zwei-Phasen-These, die davon ausgeht, daß die Telematik in der ersten Phase die prosperierenden Agglomerationen begünstigt. Die großräumige Konzentration in den Agglomerationsräumen wird bei gleichzeitiger kleinräumlicher Dezentralisierung voranschreiten. Ein Abbau der Disparitäten zwischen den strukturstarken und strukturschwachen Räumen und dem Nord-Süd-Gefälle zwischen den Agglomertionen wird mittelfristig nicht erwartet. In der zweiten Phase sollen die Vorteile einer weitergehenden Dezentralisierung zum tragen kommen, die primär auf Kleinund Mittelstädte im ländlichen Raum konzentriert ist, in denen technologisch hochwertige Produktion in einer Umwelt

147 Vgl. Spehl, H.: Räumliche wirkungen der Telematik, a.a.O., s. 261 .

$148 \mathrm{Vgl}$. beispielsweise Fritsch, M.; Ewers, H.: Telematik und Raumentwicklung, a.a.o..

$149 \mathrm{Vgl}$. Sandvoß, J.: Fernarbeit: Formen und Potentiale dezentralisierter Arbeit im Lichte qualifikations- und regionalpolitischer Aspekte, a.a.0., S. 238. 
mit entsprechendem Ambiente, Freizeitgestaltungsmöglichkeiten und attraktiven Wohn- und Arbeitsumgebungen möglich ist. 150

\subsubsection{Beschäftigungseffekte als Bestimmungsfaktor regio- nalpolitischer wirkungen}

Die neuen telekommunikativen Techniken bewirken nicht direkt die o.a. raumstrukturellen Effekte, sondern vermitteln diese über unterschiedliche 'Wirkungsfelder' und deren gegenseitige Verknüpfung. Von besonderer räumlicher Bedeutung sind

- die Beschäftigungswirkungen,

- die Standortwirkungen,

- die Flächenwirkungen und

- die Verkehrswirkungen. 151

Im folgenden werden exemplarisch die raumstrukturellen Konsequenzen der Beschäftigungswirkungen dargestellt, da $\mathrm{Ab}-$ schätzungen für die anderen Bereiche zum gegenwärtigen Zeitpunkt kaum möglich sind. Aufgrund der existierenden Analogien, zwischen der Diffusion von Industrierobotern und der Diffusion telekommunikativer Techniken, wird die These vertreten, daß die qualitative Entwicklung der Beschäftigungswirkungen von Industrierobotern und telekommunikativen Techniken Parallelen aufweist.

$150 \mathrm{Vgl.} \mathrm{Henckel,} \mathrm{D.} \mathrm{u.a.:} \mathrm{Informationstechnologie} \mathrm{und}$ Stadtentwicklung, a.a.0., S. $64 \mathrm{ff}$; Bullinger, D.: Räumliche Auswirkungen neuer Informations- und Kommunikationstechnologien, Diskussionspapier zur Herbstsitzung der DGS-Sektion Stadt- und Regionalsoziologie, 17. -20.11 .1983 , Basel 1983, S. 5 .

$151 \mathrm{Vgl}$. Henckel, D. u.a.: Informationstechnologie und stadtentwicklung, a.a.0., s. 67. 


\subsubsection{Analogien zur Diffusion von Industrierobotern}

Die Informations- und Kommunikationstechniken sind neben ihrer Funktionsprägung als Medien der Individual- und Massenkommunikation gleichermaßen als Produktions-, Organisations- und Distributionstechnologien einsetzbar. 152 Dabei kann die Anwendung im Bereich Produktion sehr viel stärkere Beschäftigungsauswirkungen haben, als die Anwendung als Raumüberwindungstechnologie. 153

Neben der Interpretation der neuen Telekommunikationstechniken als produktionstechnologie, ergeben sich weitere Analogien des Einsatzes neuer Informations- und Kommunikationstechniken im Bürobereich, wo Parallelen zur Entwicklung der Rationalisierung in der gewerblichen produktion vorliegen. Im Bürobereich ist eine Erhöhung der Arbeitsintensität festzustellen, wobei sich fließbandähnliche strukturen durchsetzen und eine zentrale Kontrolle möglich ist. Die resultierenden, von den telekommunikativen Techniken verursachten, quantitativen und qualitativen Beschäftigungseffekte, die mit einer zunehmenden Tendenz zur kapitalintensiven Produktion einhergehen, lassen sich mit den Effekten im Industrieroboterbereich vergleichen. 154

Beschäftigungswirkungen der Diffusion von Industrierobotern Im Rahmen der Meta-Studie 'Beschäftigungsrisiko Innovation?' wurden die Arbeitsmarktwirkungen neuer Technologien

$152 \mathrm{Vgl}$. Läpple, D.: Neue Technologien in räumlicher Perspektive, in: Informationen zur Raumentwicklung, Heft 4,1989, S. 214 .

$153 \mathrm{Vgl}$. ebenda.

$154 \mathrm{Vgl}$. Schütte, G.: Neue Technik, Raumordnung und Städtebau: Mögliche Auswirkungen von Telekommunikation und Mikroprozessoren auf Planung und Entwicklung von stadtund Regionalstrukturen - Tagungsbericht -, in: Raumforschung und Raumordnung, Heft 4, 41. Jahrgang, 1983 S. $170 \mathrm{ff}$. 
untersucht. 155 Mit Hilfe der dynamischen Input-outputAnalyse 156 wurde, unter Berücksichtigung der gesamtwirtschaftlichen Lieferverflechtungen, empirisch untersucht, welche Beschäftigungseffekte durch den Einsatz von Industrierobotern verursacht werden.

Abbildung 1.6 stellt die ermittelten Beschäftigungswirkungen dar. Die direkten und indirekten 'Herstellereffekte' sind positiv und die direkten und indirekten 'Anwendereffekte' negativ, im Verhältnis von ca. eins zu sieben. "Bei Berücksichtigung der durch die preissenkung verursachten Nachfrageerhöhung wird jedoch ein großer Teil der beschäftigungsmindernden wirkungen wieder kompensiert."157 Die Nachfragesteigerungen können den Beschäftigungsrückgang jedoch nicht voll kompensieren, so daß insgesamt eine Abnahme der Beschäftigten zu konstatieren ist.

Eine zusätzliche Kompensation der Rationalisierungseffekte durch Verschiebung der Nachfrage zu Sektoren mit niedriger Produktivitätssteigerung, wie beispielsweise Teile der Dienstleistungen, hat in den letzten Jahrzehnten nicht in beschäftigungssicherndem Ausmaß stattgefunden und auch für die zukunft wird eine Reduzierung des insgesamten Arbeitsvolumens erwartet. 158

155 Vgl. Matzner, E. u.a.: Beschäftigungsrisiko Innovation? - Arbeitsmarktwirkungen moderner Technologien; Befunde aus der Meta-studie, a.a.0., s. 88 .

$156 \mathrm{Vgl}$. Leontief, W.; Duchin, F.: The Future of Automation on Workers, Cambridge 1986.

157 Vgl. Matzner, E. u.a.: Beschäftigungsrisiko Innovation? - Arbeitsmarktwirkungen moderner Technologien; Befunde aus der Meta-Studie, a.a.0., s. 90.

$158 \mathrm{Vgl}$. ebenda S. 92. 
Abbildung 1.6: Beschäftigungswirkungen der Diffusion von Industrierobotern 159

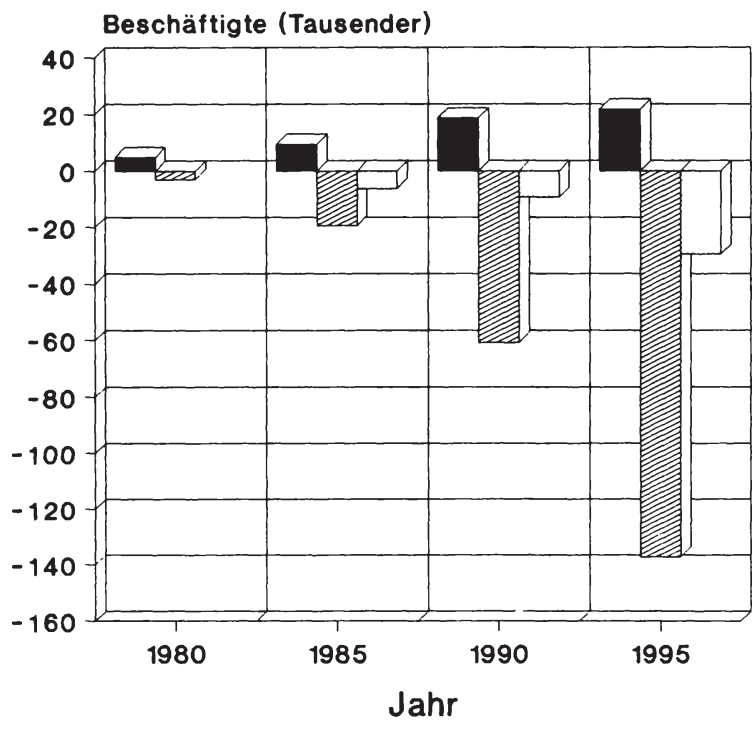

\subsubsection{Regionalpolitische Ronsequenzen der Telematikdif- fusion}

Ausgehend von der These, daß die o.a. Beschäftigungseffekte in ähnlicher Form im Bereich der Telekommunikation auftreten, kommt es, durch die unterschiedliche räumliche Zuordnung der Betroffenen, zu regionalen Disparitäten.

159 In Anlehnung an Matzner, E. u.a.: Beschäftigungsrisiko Innovation? - Arbeitsmarktwirkungen moderner Technologien; Befunde aus der Meta-studie, a.a.O., S. 90. 
Die räumliche Verteilung von Unternehmen der Informationstechnologie läßt, trotz ihrer Heterogenität, einige generelle schlußfolgerungen $\mathrm{zu} \mathbf{0}^{160}$

- Die Hersteller und Dienstleistungsunternehmen im Bereich Informationstechnik sind sehr stark auf die günstig strukturierten ${ }^{161}$ verdichtungsräume konzentriert.

- Die sủddeutschen Verdichtungsräume - insbesondere Frankfurt, stuttgart und München - sind am stärksten vertreten.

- "Neben Bundesländern mit relativ gleichmäßiger 'Technologiedichte', wie Nordrhein-Westfalen oder Baden-Württemberg, gibt es extreme Konzentrationen in Bayern, Hessen und Niedersachsen, wo die Schwerpunkte nur auf einen oder wenige Verdichtungsräume in einer technologischen 'Wüste' beschränkt bleiben."162

- Die vorhandenen Informationen deuten auf eine Verstärkung der gegenwärtigen Verteilungsmuster zugunsten der süddeutschen Räume hin.

Die direkten und indirekten, positiven Herstellereffekte, die sich auf die Telematik-Industrie, die Telematik-Dienst-

$160 \mathrm{Vgl.}$ Grabow, B.; Henckel, D.: Räumliche Verteilung von Unternehmen der Informationstechnologie, in: Akademie für Raumforschung und Landesplanung (Hrsg.): Räumliche Wirkungen der Telematik, Hannover 1987, S. 270.

161 Nach der Einteilung der Regionstypen durch die Bundesforschungsanstalt für Landeskunde und Raumordnung in:

- hochverdichtete Regionen mit günstiger Arbeitsmarktstruktur,

- altindustrialisierte Regionen mit ungünstiger Arbeitsmarkt-struktur,

- Berlin,

- Regionen mit Verdichtungskern und mittlerer siedlungsdichte,

- ländliche Regionen mit ungünstiger Arbeitsmarktstruktur und

- ländliche Regionen mit günstiger Arbeitsmarkt-Struktur (Alpenvorland).

162 Grabow, B.; Henckel, D.: Räumliche Verteilung von Unternehmen der Informationstechnologie, a.a.0., s. 270. 
leistungsunternehmen, die Deutsche Bundespost und deren $\mathrm{Zu}-$ lieferer konzentrieren, verbleiben hauptsächlich in den klassischen Ballungsgebieten.

Die Analyse der räumlichen Verteilungsmuster von Anwendern der Informationstechnologie läßt sich anhand von vier, zunächst raumunabhängigen, Diffusionstypen darstellen:

- der unternehmenseigenen Adoption,

- der Adoption durch mit 'Schlüsselunternehmen' verbundene, selbständige Unternehmen,

- den Hauptverwaltungsentscheidungen als Vorgabe für Zweigstellen sowie

- der Adoption durch öffentliche Träger. 163

In der Anfangsphase der Telekommunikationsdiffusion haben die Schlüsselunternehmen und Hauptverwaltungsentscheidungen für die räumlichen Adoptionsmuster eine prägende Rolle eingenommen. Aufgrund dessen, daß die Standorte dieser Innovatoren, einschließlich der öffentlichen Träger, bevorzugt in den Verdichtungsräumen angesiedelt sind, kann daraus die Konzentration der Entwicklung in den Verdichtungsräumen, bzw. auf die bereits existierenden Verflechtungsräume der Innovatoren, abgeleitet werden. 164

Eine Untersuchung im Rahmen des Projekts Media Park Köln für die Besatzzahlen der Bereiche "Medien" und "Telematik" unterstützt diese Einschätzung. Der Anteil der sozialversicherungspflichtigen Beschäftigten in beiden Branchen stieg insgesamt an; "dies trifft jedoch nur für die qualifizierten Tätigkeitsfelder zu: z.B. Tongerätemechaniker +11\%, Elektrogerätebauer $+14 \%$, Datenverarbeiter $+12 \%$, gleichzeitig starke Abnahme bei Telefonisten $-11 \%$ und Da-

163 Vgl. Gräf, P.: Analyse räumlicher Verteilungsmuster von Anwendern in der Bundesrepublik Deutschland, in: Akademie für Raumforschung und Landesplanung (Hrsg.): Räumliche Wirkungen der Telematik, Hannover 1987, S. 363.

$164 \mathrm{Vgl}$. ebenda. 
tentypisten -9\%; d.h. einfache Berufe - insbesondere von Frauen besetzt - verschwinden zugunsten von qualifizierten Tätigkeiten." 165

Weiterhin gibt es Hinweise, wonach künftig den Anwenderstandorten ein stärkerer Bedeutungszuwachs als den Produktionsstandorten zukommt. Hinweise in diese Richtung sind:

- der steigende Bedarf und das starke Wachstum von Software-, System-, Beratungs- und Schulungsleistungen,

- der steigende Bedarf an integrierten Anwendungslösungen und Mehrwertdiensten sowie

- die Informatisierung der produzierenden Bereiche. 166

Wahrscheinlich werden sich die Telematik-Dienstleistungsunternehmen bei ihrer Standortwahl nach den Anwendern orientieren, was zu einer weiteren Konzentration in den Agglomerationen bzw. Agglomerationsrändern führen wird.

Eine definitive räumliche Zuordnung der erwarteten Beschäftigungseffekte auf die Verdichtungsräume, deren Randzonen und auf die peripheren Regionen ist zum gegenwärtigen Informationsstand nicht möglich. Unter dem Aspekt des momentanen Diffusionsstandes, erscheint eine Konzentration der direkten und indirekten Beschäftigungswirkungen auf die günstig strukturierten Verdichtungsräume als möglich, was langfristig eine negative Beschäftigungsentwicklung für die peripheren Regionen zur Folge hätte. 167

Da über die Beschäftigungswirkungen der Diffusion von neuen

165 Küpper, U.: Aktive lokale standortentwicklung im strukturwandel, Initiativen in Köln 1983-1988, in: Informationen zur Raumentwicklung, Heft 4, 1989, S. 270.

$166 \mathrm{Vgl}$. ebenda S. 271 .

167 Vgl. Matzner, E. u.a.: Beschäftigungsrisiko Innovation? - Arbeitsmarktwirkungen moderner Technologien; Befunde aus der Meta-studie, a.a.0., S. 121. 
Telekommunikationstechniken keine empirischen Erhebungen bezüglich Hessens vorliegen, soll mit der Untersuchung des Verfassers, in Form einer primärempirischen Erhebung, analysiert werden, inwieweit Telekommunikation als standortfaktor raumstrukturelle Effekte motiviert, inwieweit ein 'Agglomerations $\leftrightarrow$ strukturschwache Regionen' Gefälle bei der Diffusion telekommunikativer Techniken existiert und inwieweit die Charakteristika der standorte einen substantiellen Einfluß auf das verhalten der potentiellen Anwender haben.

Nachdem in den bisherigen Ausführungen die Grundlagen, Entwicklungsstrukturen und erwarteten Effekte der Telematik erläutert wurden, wird im folgenden Kapitel die Telekommunikationsförderung aus der Perspektive einer regionalen wirtschaftspolitik dargestellt. 


\section{Telekommunikationsförderung aus der Perspektive der re- gionalen wirtschaftspolitik}

\subsection{Aspekte der regionalen Wirtschaftspolitik}

\subsubsection{Grundsätze und Koordinationsproblematik}

\subsubsection{2um Begriff}

Die Begriffe Regionalpolitik, regionale Wirtschaftspolitik, regionale Wirtschaftsförderung ${ }^{1}$ und regionale strukturpolitik werden in der Literatur vielfach synonym verwendet. Da Regionalpolitik vorwiegend unter einem ökonomischen Aspekt betrachtet wird ${ }^{2}$, was der Betrachtungsweise in dieser Arbeit entspricht, wird im folgenden Regionalpolitik im sinne einer regionalen wirtschaftspolitik definiert. Was jedoch nicht bedeuten soll, das regionale Wirtschaftspolitik allein der Wirtschaftspolitik zuzurechnen ist.

Die regionale Wirtschaftspolitik wird heute definiert als "der Versuch, über einen räumlich differenzierten Mitteleinsatz eine effizientere Durchsetzung gesamtwirtschaftlicher zielsetzungen $z u$ erreichen"3. "Regionale Wirtschaftspolitik wird demnach nicht verstanden als isolierte Gestaltung und/oder Beeinflussung eines Teilraumes der Volkswirt-

1 Vgl. Ewringmann, D. u.a.: Die Gemeinschaftsaufgabe 'Verbesserung der regionalen wirtschaftsstruktur' unter veränderten Rahmenbedingungen, Berlin 1986, S. 1.

2 Vgl. Brösse, U.: Ziele in der Regionalpolitik und in der Raumordnungspolitik, Berlin, New York 1972, S. 26.

3 Klemmer, P.: Zur Begründung einer regionalen wirtschaftspolitik im Rahmen der Marktwirtschaft, in: das wirtschaftsstudium (wisu), 6. Jahrgang, 1977, S. 120. 
schaft, sondern sie stellt die teilräumliche Ausprägung der nationalen wirtschaftspolitik dar." 4

Spezifisch definierte Regionseinheiten bilden für die regionale wirtschaftspolitik den räumlichen Bezugsrahmen. Ausgehend von den sozioökonomischen Besonderheiten der einzelnen Regionen, soll mit regionsspezifischen wirtschaftspolitischen Maßnahmen die Entwicklung in den einzelnen Regionen so gestaltet werden, daß ein effizienter, räumlich differenzierter Wirtschaftsprozeß initiiert wird, der dem Leitbild der nationalen Wirtschaftspolitik entspricht. 5

\subsubsection{8trategien und ziele}

Die durch wirtschaftspolitische Argumente begründete Notwendigkeit staatlicher Eingriffe in das raumwirtschaftliche Gefüge der Bundesrepublik, richtet sich in erster Linie auf die Aufhebung regionaler Disparitäten. ${ }^{6}$ Die zur Verfügung stehenden Instrumente beschränken sich auf systemkonforme Mittel, die sich vor allem aus indirekten steuererungsinstrumenten zusammensetzen, da gegenüber dem privaten sektor aufgrund der Verfassungsordnung, eine direkte steuerung nur in begrenztem Maße möglich ist. 7

4 Tennagels, P.: Instrumentarium der regionalen wirtschaftspolitik, Bochum 1980, S. 10.

5 Vgl. d'orville, H.: Probleme einer Erfolgskontrolle regionalpolitischer Maßnahmen, Frankfurt, Bern, Las Vegas 1979 , S. 25.

$6 \mathrm{Vgl}$. Hopfinger, H.: Erfolgskontrolle regionaler Wirtschaftsförderung, Erlangen 1982, S. 157.

7 Vgl. Scharpf, F.; Schnabel, F.: Steuerungsprobleme der Raumplanung, Beiträge der Akademie für Raumforschung und Landesplanung Band 27, Hannover 1979, S.9. 
Ehe die ziele der regionalen Wirtschaftspolitik im einzelnen dargestellt werden, soll analysiert werden, welche alternativen strategien der regionalen Wirtschaftspolitik zur Verfügung stehen, da die Strategien per se die Auswahl der Mittel bestimmen, die für die Verwirklichung der festgelegten ziele zum Einsatz kommen.

\section{strategien}

Zur Diskussion stehen drei strategiekonzepte:

- die passive Sanierung,

- die aktive Sanierung und

- die Politik der dezentralen Konzentration. 8

Ziel aller drei strategien ist die Beseitigung einer suboptimalen räumlichen Verteilung der Produktionsfaktoren, jedoch mit unterschiedlichen Instrumenten.

Bei der passiven Sanierung wird auf bewußte raumstrukturerhaltende Eingriffe verzichtet. Besteht in einer Region ein Überangebot an Produktionsfaktoren, so wird ausgehend von den Selbstheilungskräften des Marktes mit einer Abwanderung von Produktionsfaktoren (z.B. Arbeitskräften) in Regionen mit relativer Produktionsfaktorenknappheit gerechnet. Eine regionale Wirtschaftspolitik, die diesem Konzept entspricht, duldet passiv, fördert oder steuert eine Entwicklung des sogenannten 'moving people to the job'.9

Die aktive Sanierung soll die Wirtschaftskraft der entsprechenden Problemregion steigern, eine Abwanderung verhindern und das Wirtschaftswachstum stabilisieren. Dazu muß ein Kapitalfluß in die Problemregionen gelenkt werden, wobei, neben den indirekten 'Incentives', die Förderung von Investi-

8 Vgl. d'orville: Probleme einer Erfolgskontrolle regionalpolitischer Maßnahmen, a.a.0., S. 113.

9 Vgl. Hopfinger, H.: Erfolgksontrolle regionaler Wirtschaftsförderung, a.a.0., S. 158. 
tionskapitalverlagerungen in bestimmte Regionen, mit Hilfe direkter Finanzhilfen, empfohlen wird. Zusätzlich können diese Maßnahmen durch eine Verbesserung der Infrastruktur der betroffenen Region flankiert werden. Diese strategie des sogenannten 'moving jobs to the people' ist mit einem erheblichen Einsatz öffentlicher Mittel verbunden.10

Aufgrund der jeweiligen extremen Einstellung der beiden strategien, ergeben sich unter gesellschaftspolitischer und volkswirtschaftlicher Betrachtung erhebliche negative Konsequenzen. Die passive Sanierung birgt die Gefahr einer sozialen Erosion der Problemregionen durch die Abwanderung jüngerer und qualifizierter Arbeitskräfte, verbunden mit überproportionalen Versorgungskostensteigerungen in den Agglomerationen. Dagegen verursacht die aktive Sanierung kurzfristig erhebliche gesamtwirtschaftliche Wachstumsbehinderungen, da die optimale Faktorkombination (=größtmögliche wachstumssteigerung) zu Gunsten der Problemregionen nicht angestrebt werden kann.

Da beide strategien in ihrer Urform zu einem volkswirtschaftlichen Verlust führen, soll das Konzept der dezentralen Konzentration die Vorteile beider strategien verbinden und deren Nachteile weitgehend vermeiden. 11

Bei der strategie der dezentralen Konzentration werden öffentliche Finanzmittel auf Entwicklungsschwerpunkte, sogenannte 'growth poles' konzentriert, die Ausstrahlungseffekte auf das regionale Entwicklungspotential ausüben. Die Schwerpunktorte sollen zu einem Katalysator werden und somit einen sich selbst tragenden wirtschaftsaufschwung auslösen. "Eine strategie der dezentralen Konzentration

$10 \mathrm{Vgl}$. d'orville: Probleme einer Erfolgskontrolle regionalpolitischer Maßnahmen, a.a.o. S. 115.

11 Vgl. Lauschmann, E.: Grundlagen einer Theorie der Regionalpolitik, Hannover 1976, S. $239 \mathrm{ff}$. 
zielt auf eine aktive Sanierung im großen, bei passiver Sanierung im kleinen". 12

\section{ziele}

Nachdem, wie o.a., die Regionalpolitik als übergeordnetes Leitziel die schaffung einer optimalen regionalen wirtschaftsstruktur hat, werden im folgenden tiefere Ebenen der zielhierarchie dargestellt.

Die 'Grundsätze der regionalen Wirtschaftspolitik'13 definieren die zielsetzung der regionalen wirtschaftspolitik explizit.

\section{zielsetzung}

"Allgemeines ziel der regionalen Wirtschaftspolitik ist es, eine optimale regionale wirtschaftsstruktur zu schaffen und in allen Gebieten dafür zu sorgen, daß ungenutzte bzw. schlecht genutzte produktionsfaktoren für das allgemeine wirtschaftswachstum mobilisiert werden. Durch die regionale wirtschaftspolitik, die sich in die zielsetzungen der allgemeinen wirtschaftspolitik einfügt, wird die wirtschaftskraft in den $z u$ begünstigenden Räumen gesteigert. Es werden bessere Arbeits- und Einkommensmöglichkeiten geschaffen und dadurch auch soziale Härten dauerhaft beseitigt, die nicht nur in den wirtschaftsschwachen Räumen, sondern auch in Verdichtungsgebieten auftreten können."14

Obwohl diese zielsetzungen in ihrer Aussage bereits exakter sind, lassen sie sich Aufgrund ihrer Komplexität und ihres

12 D'orville: Probleme einer Erfolgskontrolle regionalpolitischer Maßnahmen, a.a.o. S. 117.

$13 \mathrm{Vgl}$. Deutscher Bundestag: Grundsätze der regionalen wirtschaftspolitik, Bundestagsdrucksache V/2469, Bonn 1968 , S. 5 .

14 Ebenda. 
leerformelhaften charakters ${ }^{15}$ nicht operationalisieren ${ }^{16}$. Diesbezügliche Diskussionen in Wissenschaft und Praxis resümieren drei wesentliche Teilbereiche, Wachstum, stabilität und Gerechtigkeit als allgemeine wirtschaftspolitische Zielsetzungen, 17 die zwar komplex, jedoch auf einem niedrigeren Abstraktionsniveau angesiedelt sind.

An dieser stelle soll darauf hingewiesen werden, daß die fehlende Zielkonkretisierung im politischen Prozeß eine wichtige Funktion erfüllt.l Neben der Unsicherheit über zukünftige Entwicklungen und daraus resultierenden Einflußnahmen, besteht ein Interpretationsspielraum in bezug auf angestrebte ziele, der die Involvierung der mitspracheberechtigten Gebietskörperschaften $\mathrm{zu}$ zielformulierungen erleichtert. 18 Zusätzlich wird die Konzipierung kurzfristiger und pragmatischer Handlungsprogamme gegenüber langfristigen zielformulierungen begünstigt.

Die o.a. Leitziele Wachstum, stabilität und Gerechtigkeit stellen Grundkonzepte einer Regionalpolitik dar, die, je nach den konkreten Gegebenheiten, beziehungsweise dem wirtschaftlichen Entwicklungsstand und den räumlichen Disparitäten, favorisiert werden. Grundsätzlich kann somit zwischen wachstumsorientierter, stabilitätsorientierter und versorgungsorientierter Regionalpolitik unterschieden werden. 19

$15 \mathrm{Vgl.} \mathrm{Brösse,} \mathrm{U.:} \mathrm{Ziele} \mathrm{in} \mathrm{der} \mathrm{Regionalpolitik} \mathrm{und} \mathrm{in}$ der Raumordnungspolitik, a.a.0, S. 26.

16 Vgl. Bölting, H.: Wirkungsanalyse der Instrumente der regionalen Wirtschaftspolitik, Münster 1976, S. 13.

$17 \mathrm{Vgl}$. Tennagels, P.: Instrumentarium der regionalen wirtschaftspolitik, a.a.0, s. 10.

$18 \mathrm{Vgl}$. Spehl, H. u.a.: Regionale Wirtschaftspolitik und regionale Entwicklungsplanung, Bonn 1981, S. 30.

19 Vgl. Lauschmann, E.: Grundlagen einer Theorie der Regionalpolitik, a.a.o., s. 238 . 


\section{wachstumsziel}

Die wachstumspolitische zielsetzung besteht in der Mobilisierung von Wachstumsreserven in den Problemgebieten, um ein möglichst starkes Wachstum des Sozialproduktes der Gesamtwirtschaft $\mathrm{zu}$ erreichen. ${ }^{20}$ Der Produktionsfaktor Arbeitskraft soll in den einzelnen Teilräumen möglichst optimal ausgenutzt werden. Bei öffentlichen Kapitalinvestitionen ist auf eine zieladäquate Durchführung in den Problemgebieten $\mathrm{zu}$ achten. Private Investitionen unterliegen lediglich einer indirekten steuerung durch entspechende Anreizpolitiken. Eine wachstumsorientierte Regionalpolitik impliziert somit, daß der "gesamtwirtschaftliche Wachstumsproze $\beta$ eine Resultante regionaler Wachstumsprozesse ist und umgekehrt wirtschaftliche Wachstumsprozesse eine räumliche Dimension haben" 21 .

\section{8tabilitätsziel}

Die regionalpolitischen Implikationen des stabilitätszieles liegen vor allem in einer Reduzierung der konjunkturellen und strukturellen Anfälligkeit von Regionen. 22 Durch entsprechende Koordinierung der Regional- und Sektoralpolitik sollen 'ausgewogene wirtschaftsstrukturen' entwickelt und somit 'Monostrukturen' vermieden werden. 23 Voraussetzung hierfür ist eine möglichst stabile Beschäftigungs- und Einkommensentwicklung im Gesamtraum, die mittelfristig zu einer Verstetigung des gesamtwirtschaftlichen wachstumsprozesses führt.

$20 \mathrm{Vgl}$. Deutscher Bundestag: Siebzehnter Rahmenplan der Gemeinschaftsaufgabe 'Verbesserung der regionalen Wirtschaftsstruktur', Bundestagsdrucksache 11/2362, Bonn 1988, S. 7 .

21 Storbeck, D. ; Lücke, M.: Die gesellschaftspolitische Relevanz regionalpolitischer Ziele, in: Ausgeglichene Funktionsräume, Hannover 1975, S. 23.

22 Vgl. Deutscher Bundestag: Siebzehnter Rahmenplan der Gemeinschaftsaufgabe, a.a.0., s. 7 .

$23 \mathrm{Vgl}$. d'orville: Probleme einer Erfolgskontrolle regionalpolitischer Maßnahmen, a.a.0. S. 96. 


\section{Gerechtigkeitsziel}

Die Verminderung extremer räumlicher Disparitäten hinsichtlich der Möglichkeit der Einkommenserzielung und der Versorgung mit öffentlichen und privaten Dienstleistungen, in Anlehnung an das im Grundgesetz niedergelegte Postulat der wahrung einheitlicher Lebensverhältnisse 24 , ist das ausgleichspolitische $\mathrm{ziel}$ der regionalen strukturpolitik. 25

Da die regionale Wirtschaftspolitik nicht alle drei ziele gleichzeitig realisieren kann, muß darüber entschieden werden, welchem der ziele Priorität gegenüber den anderen eingeräumt werden soll. Die Entscheidung ist aufgrund der fehlenden zielhierarchie nicht eindeutig und in der Literatur umstritten. 26 spehl z.B. sieht die Priorität beim wachstumsziel27, wogegen Eberstein 28 die regionalpolitischen Maßnahmen primär als Ausgleichsmaßnahmen ansieht. Letztendlich ausschlaggebend sind, neben der Gesamtkonzeption der Wirtschafts- und Gesellschaftspolitik, die aktuellen Gegebenheiten bezüglich des wirtschaftlichen Entwicklungsstandes und der räumlichen Disparitäten.

Abbildung 2.1 stellt "eine zielsystematik der heute im politischen und wissenschaftlichen Bereich intendierten re-

24 Vgl.: Artikel 72 Absatz 2 Grundgesetz und Artikel 106 Absatz 3 Grundgesetz.

$25 \mathrm{Vgl}$. Böventer von, E.: Die räumlichen Wirkungen von öffentlichen und privaten Investitionen, in: Arndt/Swatek (Hrsg.): Grundfragen der Infrastrukturplanung, Berlin 1971, S. $167 \mathrm{ff}$.

26 Vgl. d'orville: Probleme einer Erfolgskontrolle regionalpolitischer Maßnahmen, a.a.0. S. 99.

$27 \mathrm{Vgl}$. Spehl, H.: Thesenpapier, in: Regionalpolitik 2000 - Probleme, Ziele, Instrumente, Schriftenreihe der Wirtschafts- und Gesellschaftswissenschaftlichen Gesellschaft Trier e.V., Trier 1984, S. 16.

$28 \mathrm{Vgl}$. Eberstein, H.: Grundlagen der Regionalpolitik und ihre wesentlichen Grundsätze, in: Handbuch der regionalen Wirtschaftsförderung, Köln ab 1971, Abschnitt A III, S. 15 . 
gionalen Wirtschaftspolitik"29 dar. Basis hierfür waren die regionalen Raumordnungspläne, das Landesentwicklungsprogramm und das Bundesraumordnungsprogramm sowie die entsprechenden Fachpolitiken. Ausgehend vom oberziel 'Schaffung einer optimalen regionalen Wirtschaftsstruktur', wird über die drei Hauptziele hin ein Indikatorensystem vorgeschlagen. Relevant werden einige dieser Indikatoren bei der Definition der Fördergebiete, für die sich als schwellenwerte die Bundes- und Landesdurchschnitte, die Abweichungen vom Durchschnitt der Fördergebiete/Nichtfördergebiete und die Vorschläge des Beirates für Raumordnung anbieten.

29 Spehl, H. U.a.: Regionale Wirtschaftspolitik und regionale Entwicklungsplanung, a.a.O., S. 38 . 
Abbildung 2.1: Intendiertes Zielsystem der praktizierten regionalen Wirtschaftspolitik 30

\begin{tabular}{|c|c|c|}
\hline & $\begin{array}{l}\text { Schaffung einer optimalen } \\
\text { regionalen Wirtschafsstruktur }\end{array}$ & tur \\
\hline Wachstumsziel & stabilitätsziel & Gerechtigkeitsziel \\
\hline $\begin{array}{l}\text { Regionale Beiträge } \\
\text { zum Wachstum der Ge- } \\
\text { samtwirtschaft durch } \\
\text { optimale Allokation } \\
\text { der Produktionsfak- } \\
\text { toren in den einzel- } \\
\text { nen Regionen }\end{array}$ & $\begin{array}{l}\text { Stabile Beschäfti- } \\
\text { gungs und Einkommens- } \\
\text { entwicklung der Ge- } \\
\text { samtwirtwirtschaft } \\
\text { durch Reduktion der } \\
\text { konjunkturellen und } \\
\text { strukturellen Anfäl- } \\
\text { ligkeit der regiona- } \\
\text { len standort- und } \\
\text { Produktionsstrukturen }\end{array}$ & $\begin{array}{l}\text { Schaffung gleichwer- } \\
\text { tiger Lebensbedin- } \\
\text { gungen durch Reduzie- } \\
\text { rung interregionaler } \\
\text { Einkommensgefälle }\end{array}$ \\
\hline $\begin{array}{l}\text { Physische Infra- } \\
\text { struktur }\end{array}$ & $\begin{array}{l}\text { Saisonale Arbeitslo- } \\
\text { sigkeit }\end{array}$ & $\begin{array}{l}\text { Bruttoarbeitsentgeld } \\
\text { je sozialversiche- } \\
\text { rungspflichtig be- } \\
\text { schäftigten AN }\end{array}$ \\
\hline $\begin{array}{l}\text { Arbeitskräftereser- } \\
\text { vequotient }\end{array}$ & $\begin{array}{l}\text { Strukturelle Ar- } \\
\text { beitslosikgeit }\end{array}$ & BIP/Beschäftigte \\
\hline $\begin{array}{l}\text { Spezialisationsko- } \\
\text { effizient stark re- } \\
\text { zessiver Industrie- } \\
\text { zweige }\end{array}$ & $\begin{array}{l}\text { Wanderungssaldo } \\
\text { Anteil der Ange- } \\
\text { stellten bzw. Fach- } \\
\text { arbeit an den Be- } \\
\text { schäftigten }\end{array}$ & $\begin{array}{l}\text { BIP/Wirtschaftsbe- } \\
\text { völkerung } \\
\begin{array}{l}\text { Lohn- und Gehalts- } \\
\text { summe/Beschäftigte }\end{array}\end{array}$ \\
\hline
\end{tabular}

30 In Anlehnung an: Spehl, H. U.a.: Regionale wirtschaftspolitik und regionale Entwicklungsplanung, a.a.o., s. 40. 


\subsubsection{Rechtliche Grundlagen}

Die regionale Wirtschaftspolitik, als räumlich differenzierter Teilbereich der Wirtschaftsstrukturpolitik, ist in ein übergreifendes Raumordnungskonzept und die globale Wirtschaftspolitik des Bundes und der Länder eingebunden. 31 Gemäß Artikel $30 \mathrm{bzw}$. Artikel 28 des Grundgesetzes liegt die Regionalpolitik grundsätzlich in der zuständigkeit der Länder bzw. der Gemeinden, die diese u.a. in Form von landeseigenen regionalen Wirtschaftsförderungen wahrnehmen.

Gemäß Artikel 91 a des Grundgesetzes, der im Jahre 1969 in das Grundgesetz der Bundesrepublik Deutschland eingefügt und 1970 bezüglich der regionalen Wirtschaftspolitik konkretisiert wurde, verpflichtet sich der Bund, im Rahmen der Gemeinschaftsaufgabe 'Verbesserung der regionalen Wirtschaftsstruktur', an der regionalen Wirtschaftsförderung der Länder mitzuwirken. Die Durchführung der Gemeinschaftsaufgabe obliegt ausschließlich den Ländern.

Die konkreten Fördermaßnahmen einer regionalen Wirtschaftspolitik werden durch folgende Bezugspunkte determiniert:

- das Gesetz über die Gemeinschaftsaufgabe 32

'Verbesserung der regionalen Wirtschaftsstruktur',

- das Investitionszulagengesetz ${ }^{33}$,

- das Zonenrandförderungsgesetz ${ }^{34}$,

- das European Recovery Program (ERP) ${ }^{35}$,

31 Vgl. Ewringmann, D. u.a.: Die Gemeinschaftsaufgabe 'Verbesserung ..., a.a.0., s. 1 .

32 Abgedruckt im Anhang $\mathrm{F}$ des 17. Rahmenplanes der Gemeinschaftsaufgabe 'Verbesserung der regionalen Wirtschaftsstruktur', Bundestagsdrucksache 11/2362, Bonn 1988 .

33 Abgedruckt ebenda im Anhang $J$.

34 Abgedruckt ebenda im Anhang G.

35 Abgedruckt ebenda im Anhang $K$ und $L$. 
- die Förderprogramme der Länder und sonstige Förderungen sowie

- den Europäischen Fonds für regionale Entwicklung (EFRE). 36

\section{Gemeinschaftsaufgabe}

Nach dem Gesetz über die Gemeinschaftsaufgabe werden Fördermaßnahmen durchgeführt:

- im gesamten Zonenrandgebiet,

- in Regionen, deren Wirtschaftskraft erheblich unter dem allgemeinen Durchschnitt liegt bzw. zukünftig absinken könnte und

- in Regionen, in denen Wirschaftszweige dominieren, die vom strukturwandel so stark betroffen sind, daß davon negative Rückwirkungen auf das Wirtschaftsleben der gesamten Region $\mathrm{zu}$ erwarten sind. 37

Die Fördermaßnahmen in den o.a. Problemregionen sollen anhand von finanziellen Anreizen, in Form von Investitionszuschüssen, Darlehen, Zinszuschüssen und Bürgschaften, durchgeführt werden. Die finanziellen Anreize können für folgende Maßnahmen gewährt werden:

- die Errichtung, Erweiterung, Umstellung oder grundlegende Rationalisierung von gewerblichen Betrieben,

- den Ausbau der wirtschaftsnahen Infrastruktur, einschließlich der Industriegeländeerschließung und

- die Förderung wirtschaftsnaher Bildungssektoren und des Fremdenverkehrs. 38

36 Abgedruckt ebenda im Anhang $M$.

$37 \mathrm{Vgl}$. Bundesministerium für Wirtschaft (Hrsg.): Regionale wirtschaftsförderung in der Bundesrepublik Deutschland, Bonn, Duisdorf 1989, S. 5.

$38 \mathrm{Vgl}$. Deutscher Bundestag: Siebzehnter Rahmenplan der Gemeinschaftsaufgabe, a.a.0., s. $28 \mathrm{ff}$. 
Zusätzlich werden die Fördermaßnahmen auf räumliche und sachliche Schwerpunkte konzentriert, die sich aus dem jährlich fortzuschreibenden Rahmenplan ergeben. Die von Bund und Ländern beschlossene kurz- und mittelfristige Finanzplanung ist in diesen Rahmenplan zu involvieren, was der Gemeinschaftsaufgabe eine zentrale regionalpolitische Bedeutung zukommen läßt. 39

\section{Investitionszulagengesetz}

Die aus der Gemeinschaftsaufgabe vergebenen Mittel stehen in zusammenhang mit anderen regionalpolitischen Fördermaßnahmen, im besonderen mit dem 1969 eingeführten Investitionszulagengesetz. Obwohl das Investitionszulagengesetz eine eigenständige Rechtsgrundlage bildet, ist es in seinem Regionalteil bezüglich der Auswahl der Fördergebiete und wichtiger Grundsätze an die Gemeinschaftsaufgabe angepaßt. 40 Die regionale Investitionszulage, die nach $\$ \$ 1-3$ Investitionszulagengesetz für gewerbliche Investitionen gewährt wird, stellt die Basisförderung der Gemeinschaftsaufgabe dar. Die Zuschüsse aus der Gemeinschaftsaufgabe ergänzen die Förderung lediglich bis zur regionalpolitisch fixierten obergrenze.

Unterschiede zur Gemeinschaftsaufgabe basieren auf der Rechtsnatur des Investitionszulagengesetzes und auf den unterschiedlichen Förderobjekten bzw. der Förderungsfähigkeit. Die zulagengewährung erfolgt nicht als öffentlicher Zuschuß, sondern unmittelbar durch das Einkommen- und Körperschaftsteueraufkommen des zuständigen Finanzamtes. Bei Nachweis der Förderungswürdigkeit besteht ein Rechtsanspruch auf die Gewährung der zulage, die steuerfrei von den Finanzbehörden ausgezahlt, bzw. mit der steuerschuld verrechnet wird. Der Zuschuß beträgt im Zonenrandgebiet $10 \%$

$39 \mathrm{Vgl}$. Hopfinger, H.: Erfolgksontrolle regionaler Wirtschaftsförderung, a.a.0., S. 163 .

$40 \mathrm{Vgl}$. Ewringmann, D. U.a.: Die Gemeinschaftsaufgabe 'Verbesserung ..., a.a.0., s. 11 . 
und im übrigen Fördergebiet $8,75 \%$ der förderfähigen Investitionskosten. Grundstückskosten bei Investitionsvorhaben und Ersatzbeschaffungen sind nicht förderfähig. Weitere Einschränkungen ergeben sich durch eine restriktive räumliche Abgrenzung der Förderstandorte.

\section{Zonenrandförderungsgesetz}

Das Gesetz zur Förderung des Zonenrandgebietes vom 5 . August 1971 enthält Sonderregelungen mit einem breiteren instrumentellen Ansatz, die zusätzlich zu den Maßnahmen der Gemeinschaftsaufgabe gewährt werden. Es umfaßt vor allem steuerliche Vergünstigungen und spezielle Förderungsregelungen zugunsten des Wohnungswesens, sozialer Einrichtungen sowie von Verkehrsmaßnahmen und Kultur- und Bildungseinrichtungen. Durch die Vorverlegung steuerlicher Abschreibungen, durch Bildung von Rücklagen oder durch Sonderabschreibungen, wird die Herstellung oder Anschaffung abnutzbarer Wirtschaftsgüter des Anlagevermögens gefördert. Zusätzlich werden Beihilfen zum Ausgleich von Frachtmehrkosten gewährt. Desweiteren sollen die Unternehmen des Zonenrandes bei der öffentlichen Vergabe von Aufträgen besonders berücksichtigt werden.

\section{European Recovery Program}

Die Förderungen im Rahmen des ERP-Regionalprogramms stellen eine Ergänzung der Gemeinschaftsaufgabe dar. Aus dem ERPSondervermögen können Darlehen an kleine und mittlere Unternehmen im Fördergebiet der Gemeinschaftsaufgabe vergeben werden, die nicht die Fördervoraussetzungen für die regionale Investitionszulage oder den Investitionszuschuß nach dem Rahmenplan erfüllen. Die Förderung wird in Form von Darlehen $\mathrm{zu}$ vergleichsweise günstigen Konditionen gewährt und soll für Investitionen im Bereich der Errichtung, Erweiterung, grundlegenden Rationalisierung und Umstellung von Betriebsstätten gewährt werden. Bei Betriebserweiterungen soll eine angemessene Zahl neuer Arbeitsplätze geschaffen werden. 
Das ERP-Gemeindeprogramm gewährt Gemeinden in Schwerpunktorten der Gemeinschaftsaufgabe zinsbegünstigte Darlehen für wirtschaftsnahe Infrastrukturinvestitionen. Die Investitionen sollen der steigerung des Wohn- und Freizeitwertes in den Gemeinden dienen. Die mit der Förderung verbundene Aufwertung der standortqualität soll Ansiedlungen gewerblicher Unternehmen in den Schwerpunktorten erleichtern.

\section{Förderprogramme der Länder und sonstige Förderungen}

Nach Aritkel 91a Grundgesetz, ist die Verbesserung der regionalen Wirtschaftsstruktur als Länderaufgabe fixiert und die Mitwirkung des Bundes an bestimmte Voraussetzungen gebunden. Aus Artikel 91a läßt sich somit direkt das Recht der Länder ableiten, mit eigenständigen Förderprogrammen die Förderungen der Gemeinschaftsaufgabe zu ergänzen. 41 Ein Beispiel hierfür ist Bayern mit dem 'Regionalen Förderprogramm für die gewerbliche wirtschaft', das Investitionen, die der Errichtung, Erweiterung, Rationalisierung oder Modernisierung von Betrieben der gewerblichen Wirtschaft dienen, unterstützt. Die Förderung selbst ist auch in Nicht-Fördergebieten der Gemeinschaftsaufgabe möglich, was eine Ausweitung der Fördergebiete auf fast das gesamte Bundesland erlaubt. 42 zusätzlich können die Förderungstatbestände erweitert und der tertiäre Bereich stärker einbezogen werden. Aus der sicht der Gemeinschaftsaufgabe führt dies zu einer Verminderung der Präferenzen der eigenen Fördergebiete, was den räumlichen Konzentrationsprozess, der durch die Schwerpunktförderung eingeleitet werden sollte, abschwächt.

Neben den regionalen Programmen zur Wirtschaftsförderung, gibt es weitere Fördermöglichkeiten, die nicht primär auf

41 Vgl. Wild, K.-P.: Stellung und Aufgaben der Länder, in: Handbuch der regionalen Wirtschaftsförderung, a.a.0., Abschnitt A VI. 1, S. 2.

$42 \mathrm{Vgl}$. Hopfinger, H.: Erfolgksontrolle regionaler Wirtschaftsförderung, a.a.0., S. 165. 
eine Region bezogen sind, zum Beispiel das Mittelstandsförderungsprogramm.

\section{Europäischer Fonds für regionale Entwicklung}

Die nationale Regionalpoltik wird durch die EG-Regionalpolitik unterstützt und auch reglementiert. Das zentrale Finanzierungs- und Förderungsinstrument der EG-Regionalpolitik stellt der Europäische Fonds für regionale Entwicklung dar, dessen Fördermittel weitestgehend zur finanziellen Unterstützung regionaler Förderprogramme der Mitgliedsstaaten eingesetzt werden. Die auf die Bundesrepublik Deutschland entfallenden Mittel stellen einen finanziellen Beitrag für die Gemeinschaftsaufgabe dar, wobei bisher keine wesentliche konzeptionelle Beeinflussung der strategie der Gemeinschaftsaufgabe stattfindet. 43

Es besteht jedoch ein wandel in der EG-Regionalpolitik dahingehend, besonders förderungswürdige Regionen unabhängig von der Quotenregel stärker zu fördern. Zusätzlich hat sich innerhalb der Quotenregelung ein Trend zur Konzentration der Fördermittel auf Mitgliedsstaaten mit besonders gravierenden Regionalproblemen durchgesetzt. Dies impliziert mittelfristig mögliche Konsequenzen für die Konzeption und instrumentelle Ausgestaltung der Gemeinschaftsaufgabe.

\subsubsection{Roordinationsproblematik}

Die Raumordnungspolitik beinhaltet sämtliche Planungs- und Fördermaßnahmen innerhalb der Bundesrepublik Deutschland, die auf eine Verbesserung der räumlichen struktur im ökonomischen, gesellschaftlichen, kulturellen und ökologischen Bereich zielen. Die regionale Wirtschaftspolitik ist somit

$43 \mathrm{Vgl}$. Ewringmann, D. U.a.: Die Gemeinschaftsaufgabe 'Verbesserung ..., a.a.0., S. 15 . 
in ein übergeordnetes Raumordnungskonzept eingebunden und unterliegt einer Abstimmungs- und Koordinierungsverpflichtung in dem Sinne, "daß die Maßnahmen der regionalen wirtschaftspolitik in die Entwicklungsplanungen der Raumordnung integriert werden müssen"44. "Bei Zielkonflikten ist die Interessenlage gegeneinander abzuwägen und eine bestmögliche Lösung anzustreben."45 Dies impliziert jedoch keine Zielharmonie, da eine verbindliche Rangordnung der ziele nicht vorgegeben ist.

Am 30. Januar 1985 wurden von der Bundesregierung die neuen 'Programmatischen Schwerpunkte der Raumordnung' beschlossen, um zielantinomien der raumwirksamen planungen und Maßnahmen des Bundes $z u$ reduzieren. ${ }^{46}$

Um die ziele der regionalen wirtschaftspolitik nicht zu konterkarieren, ist es notwendig, die Konsistenz der anderen raumwirksamen Fachpolitiken gegenüber der regionalen Wirtschaftspolitik, über die schaffung eines gemeinsamen räumlichen Bezugsrahmens, zu stärken. Basis dieses Bezugsrahmens ist die Gemeinschaftsaufgabe. 47 Darüberhinaus übernimmt die Gemeinschaftsaufgabe weitere Koordinierungsfunktionen, die sich in drei Funktionsbereiche untergliedern lassen: 48

44 Hübner, K.-H.: Rechtsgrundlagen und Programme der Raumordnung, in: Handbuch der regionalen Wirtschaftsförderung, a.a.0, Abschnitt A IV 1, s. 2 f.

45 Deutscher Bundestag: Grundsätze der regionalen Wirtschaftspolitik, a.a.0., s. 5 .

$46 \mathrm{Vgl}$. Deutscher Bundestag: Programmatische Schwerpunkte der Raumordnung, Bundestagsdrucksache 10/3146, Bonn 1985.

47 Vgl. Deutscher Bundestag: Siebzehnter Rahmenplan der Gemeinschaftsaufgabe, a.a.0., S. 14 .

$48 \mathrm{Vgl}$. Bundesministerium für Wirtschaft (Hrsg.): Wirksame Regionalpolitik - Fortschritte in den Regionen, Bonn 1980 , S. $8 \mathrm{f}$. 
1. Eine Koordinierungsfunktion der Gemeinschaftsaufgabe besteht darin, Rahmenbedingungen auf dem Gebiet der regionalen Wirtschaftsförderung zu setzen, die sich auf die Aktivitäten von Bund, Ländern und Kommunen beziehen. Die Regeln bezüglich der Förderkonkurrenz der Länder umfassen:

- die Abgrenzung der Fördergebiete nach einem bundeseinheitlichen Verfahren,

- die bundesweit einheitlichen Rahmenbedingungen bei der Bestimmung der Schwerpunktorte,

- die Wahrung eines Präferenzgefälles bei den Förderhöchstsätzen, unter besonderer Berücksichtigung des Zonenrandgebietes und Berlin sowie

- die Schaffung einheitlicher Förderungsregelungen über Voraussetzungen, Art und Intensität der Förderung.

Im Verhältnis Bund-Länder besteht ein weiterer Koordinationsbedarf bezüglich der neben der Gemeinschaftsaufgabe existierenden Landesförderungsprogramme mit regionaler Zweckbestimmung. Hier müssen Zielantinomien vermieden werden, was für die Landesförderprogramme eine Reglementierung der Förderhöchstsätze zur Folge hat.

2. Eine zweite Koordinierungsfunktion der Gemeinschaftsaufgabe besteht in der Abstimmung mit anderen regionalwirtschaftlich relevanten Politiken, wie der Verkehrs-, Energie-, Agrar-, Städtebau-, Berufsbildungs-, Forschungs- (und) Technologie-, Umwelt- und Arbeitsmarktpolitik.

3. Der dritte Bereich bezieht sich auf die nationale regionale strukturpolitik, die mit der europäischen Regionalpolitik abgestimmt werden muß. Hier dominieren zwei Grundprobleme : 
- Bei den Mitgliedstaaten der Europäischen Gesellschaft müssen konkretisierte regionale Entwicklungsprogramme erarbeitet werden, welche die ziele, die Maßnahmen und den Finanzaufwand beinhalten. Die Gemeinschaftsaufgabe gilt auf europäischer Ebene als beispielhaft.

- Die Existenz der zusätzlichen Regionalfondsmittel muß gewährleistet sein. Dies sichert zusätzliche regionale Entwicklungsimpulse.

Für die Zukunft wird die Bedeutung grenzüberschreitender Programme zunehmen. Ein Beispiel stellt das 1978 vorgelegte deutsch-niederländische Programm für die Ems-Dollart-Region dar.

\subsubsection{Trends in den Konzepten der regionalen wirtschafts- politik}

\subsubsection{Von der exogenen zur endogenen Beeinflussung}

Die durch die gewandelten internationalen Wettbewerbsverhältnisse hervorgerufene Beschleunigung des wirtschaftlichen strukturwandels, impliziert eine Verschiebung der regionalen Problemmuster für die zukunft. Die veränderten regionalpolitischen Rahmenbedingungen führen zur Notwendigkeit einer Überprüfung der Konzepte der regionalen Wirtschaftspolitik. 49

Die Entwicklung strukturschwacher Regionen durch Impulse von 'außen' wird zunehmend schwerer. Die großräumige Verlagerung von Arbeitsplätzen geht spürbar zurück, und die Voraussetzungen für die Wettbewerbsfähigkeit der Betriebe in

$49 \mathrm{Vgl}$. Spannowsky, W.: Der Handlungsspielraum und die Grenzen der regionalen Wirtschaftsförderung des Bundes, Berlin 1987 , S. $34 \mathrm{f}$. 
strukturschwachen Regionen haben sich verändert. Vor dem Hintergrund dieser Entwicklungen sind die Konzeption und das Instrumentarium der regionalen Wirtschaftsförderung vom Bund und den Ländern überprüft und weiterentwickelt worden. 50 Trotzdem bestehen weitere darüber hinausgehende Forderungen nach einer Umorientierung, 51 die sich grundsätzlich in drei Bereiche untergliedern lassen:

1. Die Wachstums- und Beschäftigungseffekte der Regionalförderung sollen durch die stärkung der regionseigenen ökonomischen Kräfte erhöht werden. Die regionale wirtschaftspolitik soll primär auf eine Bestandssicherung und -entwicklung der in den Regionen vorhandenen Betrieben ausgerichtet werden, da in zukunft Neuanwerbungen von Unternehmen die Ausnahme darstellen werden. Die Forderung einer Ausschöpfung des vorhandenen regionsinternen Entwicklungspotentials, also nach einer 'endogenen Entwicklung' einer Region, gewinnt zunehmend an Bedeutung. 52

2. Problemregionen sind nicht mehr ausschließlich periphere ländliche Gebiete. Unterschiedlich strukturierte wirtschaftsräume mit spezifischen Schwächen werden zunehmend zu Problemregionen, was den flächendeckenden Anspruch eines einzigen Entwicklungsinstrumentes, der Kapitalsubvention, zunehmend in Frage stellt.53 "In jüngster zeit zeigt sich demgegenüber eine zunehmende

$50 \mathrm{Vgl}$. Deutscher Bundestag: Raumordnungsbericht 1986, Bundestagsdrucksache 10/6072, Bonn 1986, S. 62 .

51 Vgl. Müller, K.: Arbeitsmarktorientierte Regionalpolitik, Nr. 86 der Arbeitsmaterialien der Akademie für Raumforschung und Landesplanung, Hannover 1984, S. 134.

$52 \mathrm{Vgl}$. o.V.: Endogene Entwicklungsstrategien, in: Informationen zur Raumentwicklung, Heft $1 / 2,1984$.

$53 \mathrm{Vgl.}$ Eckey, H.-F. u.a.: Sozioökonomische und ökologische Bedingungen für die Entwicklung Nordhessens, Gesamthochschule Kassel, Kassel 1987, S. 147. 
Abkehr von generellen Konzepten dieser Art und eine gewisse Hinwendung $\mathrm{zu}$ einer problemorientierten, an (raumspezifischen) Engpässen orientierten Regionalpolitik." 54

3. Eine stärkere Ausrichtung an den regionalpolitischen zielsetzungen der betroffenen Regionen, in Richtung einer 'Regionalisierung der Regionalpolitik', führt zu der Forderung nach einer Dezentralisierung der Regionalpolitik. Im Kern beinhaltet diese Forderung die verstärkte Beteiligung der betroffenen Regionen am Entscheidungsprozeß.55 Die Förderung einer Region müßte somit unter Beteiligung regionaler Interessengruppen erfolgen, um eigene Entwicklungsvorstellungen, die aus den Besonderheiten einer Region resultieren, zu vertreten.

Die Erwägungen bezüglich der Region als Träger der regionalen Entwicklungspolitik, werden, wie o.a., zunehmend unter dem Gesichtspunkt einer verstärkt von unten, also dezentral, erfolgenden Koordinierung der Regionalpolitik mit anderen Fachpolitiken diskutiert. 56 Dabei wird nicht ein vollständiger Ersatz zentraler Maßnahmen angestrebt, sondern eine gesamtgesellschaftlich effiziente "Balance zwischen eigenständiger Regionalentwicklung und bundesstaatlicher gemeinsamer Rahmensetzung und Landesplanung" 57.

Voraussetzung einer solchen 'regionalen Regionalpolitik' ist die schaffung eines adäquaten ordnungsrahmen. Insbesondere "muß sichergestellt werden, daß die räumliche Alloka-

54 Müller, K.: Arbeitsmarktorientierte Regionalpolitik, a.a.0, S. 134 .

$55 \mathrm{Vgl}$. Spannowsky, w.: Der Handlungsspielraum und die Grenzen der regionalen Wirtschaftsförderung des Bundes, a.a.0., s. 37 .

56 Ebenda.

57 Hartke, S.: Selbstverantwortete regionale Entwicklung im 
tion nicht durch redistributive Maßnahmen des öffentlichen Sektors bzw. durch eine ruinöse Konkurrenz zwischen einzelnen Regionen verzerrt wird" 58.

\subsubsection{Von der Nachfrageorientierung hin zur Angebots- orientierung}

In der jüngeren Literatur wird die Forderung erhoben, eine Regionalpolitik verstärkt an den vorhandenen ökonomischen Kräften der strukturschwachen Regionen anzusetzen.59 Die Angebotsflexibilität, also die Anpassungsgeschwindigkeit des Angebotes an veränderte Rahmenbedingungen, soll erhöht werden (supply-theorie), was mit einer Reduzierung des antizyklischen Nachfrageverhaltens des staates verbunden ist. 60 Mit dem 14. Rahmenplan der Gemeinschaftsaufgabe sind neue Akzente für die regionale Wirtschaftspolitik gesetzt worden, die teilweise den Forderungen nach einer Umgewichtung vom nachfrage- hin zum angebotsorientierten Ansatz in der Regionalpolitik entsprechen.

Ansätze einer angebotsorientierten Regionalpolitik sind der faktororientierte Ansatz, in dem die Menge der eingesetzten Produktionsfaktoren variiert wird und der produktivitätsorientierte Ansatz, der eine Änderung der Produktivität der

Rahmen der Raumordnung, in: Raumordnung und Raumforschung, Heft 2, 1984, S. 96ff.

58 Junkernheinrich, M.: Dezentralisierung der Wirtschaftspolitik, in: Wirtschaftsdienst $\mathrm{Nr}$. XI, 1985, S. $576 \mathrm{ff}$.

$59 \mathrm{Vgl}$. Eckey, H. -F.: Regionale Engpaßfaktoren, in: Nordrhein-Westfalen in der Krise - Krise in Nordrhein-Westfalen? Schriften des Rheinisch-Westfälischen Instituts für Wirtschaftsforschung Essen, Heft 46, Berlin 1985, s. $7-37$.

$60 \mathrm{Vgl}$. Eckey, H.-F. U.a.: Sozioökonomische und ökologische Bedingungen für die Entwicklung Nordhessens, a.a.0., s. 150 . 
Arbeit und (oder) des Kapitals impliziert. Unter Berücksichtigung des Rückgangs der Bevölkerungsgröße und der Reduzierung der altersstrukturbereinigten Erwerbsquote aufgrund der Lebensarbeitszeitverkürzungen kommt der faktororientierten Regionalpolitik jedoch nur eine geringe Bedeutung zu. 61

Eine Erhöhung der Produktivität des Faktors Kapital kann durch eine innovationsorientierte Regionalpolitik erreicht werden, die Innovationen im Bereich der Entstehung, der Anwendung und des Einsatzes neuer Technologien fördert. 62

Ein weiterer Aspekt der innovationsorientierten Regionalpolitik stellt die Verzahnung mit der Bildungspolitik dar. Die Arbeitsproduktivität einer Region läßt sich durch eine verbesserte Ausbildung des Faktors Arbeit erhöhen, wobei der technologische Standard des Sachkapitals von Unternehmen im engen Zusammenhang mit der Qualifikation des Unternehmers und der Arbeitskräfte steht. 63

Eine angebotsorientierte Entwicklungspolitik, die gleichzeitig produktivitätsorientiert ist, läßt sich somit wie folgt zusammenfassen: 64

1. Eine innovationsorientierte Regionalpolitik umfaßt alle Maßnahmen der Invention und Innovation, verbunden mit der Diffusion neuen wissens, die den technologischen standard einer Region erhöhen.

$61 \mathrm{Vgl}$. ebenda.

$62 \mathrm{Vgl.} \mathrm{Genosko,} \mathrm{J.:} \mathrm{Die} \mathrm{innovationsorientierte} \mathrm{Regionalpo-}$ litik, Eine wirksame Handlungsalternative?, in: Raumforschung und Raumordnung, Heft 2/3, 1986, S. 108.

$63 \mathrm{Vgl}$. Ewers, H.-J., Wettmann, R.: Innovationsorientierte Regionalpolitik, Bonn 1980, S. $31 \mathrm{f}$.

$64 \mathrm{Vgl}$. Eckey, H.-F. U.a.: Sozioökonomische und ökologische Bedingungen für die Entwicklung Nordhessens, a.a.0., s. $152 \mathrm{f}$. 
2. Sie ist zentraler Bestandteil einer angebotsorientierten (regionalen) Wirschaftspolitik, die zunehmend an Bedeutung gewinnt. Die 'supply-theory' zielt auf eine steigerung der Anpassungsflexibilität des Angebots an veränderte Rahmenbedingungen $\mathrm{ab}$.

3. Innovation und fertigungstechnische Adaption sind vom Wissensstand der Arbeitskräfte abhängig. Dies führt zu einer Verzahnung von innovations-, bildungs- und kapitalorientierter Regionalpolitik.

4. Die Schaffung entsprechender Infrastruktur und Rahmenbedingungen ist in den Energie-, Flächen-, und Umweltbereichen erforderlich, damit entwicklungslimitierende Faktoren ausgeschlossen werden.

\subsubsection{8tandortfaktorwechsel}

Ausgehend von den Regionalwissenschaften, läßt sich die standorttheorie in einen positiven und einen normativen Aspekt untergliedern. Der normative Aspekt beschäftigt sich mit dem Problem, welchen standort ein Betrieb auswählen sollte, um nach einem vorgegebenen Maßstab ein Optimum zu erreichen. Der positive Aspekt dagegen untersucht die Frage, warum ein Betrieb einen bestimmten standort im Raum ausgewählt nat. 65

Setzt man das Streben nach maximalem Gewinn als unternehmerisches Hauptziel voraus, so müßten sämtliche kosten- und erlösrelevanten standortfaktoren von primärer Bedeutung für die standortwahl sein. Neben den rentabilitätsorientierten standortfaktoren sind jedoch auch Einflüsse aus dem priva-

65 Vgl. Eckey, H.-F. u.a.: Sozioökonomische und ökologische Bedingungen für die Entwicklung Nordhessens, a.a.0., S. $154 \mathrm{f}$. 
ten Bereich von Bedeutung. Diese Einflüsse sind z.B. die Wohn-, Freizeit- und Umweltsitutationen des Standortes, die zur letztendlichen standortwahl mit ausschlaggebend sind. Der Erfolg einer Neugründung beispielsweise wird unter anderem vom Verhalten anderer Personen, wie den leitenden Angestellten oder den Pendlern mitbestimmt. Die Integration dieser 'neuen' Ziele in die traditionellen Zielsysteme ist abhängig von der Flexibilität der Unternehmer und wird zum großen Teil erst durch negative Erfahrungen ausgelöst. 66

Die standortfaktoren lassen sich grob, wie in Abbildung 2.2 dargestellt, in zwei Gruppen mit entsprechenden Einzelfaktoren unterglieden.

Die Relevanz der einzelnen standortfaktoren ist für jedes Unternehmen unterschiedlich, dennoch kristallisieren sich aufgrund verschiedener empirischer Untersuchungen Präferenzen heraus, die auf gewisse Trends hindeuten. 67

Untergliedert man die standortfaktoren, wie in Abbildung 2.2 dargestellt, nach den die produktionsrentabilität beeinflussenden Faktoren und den Versorgungs- und Freizeitbereich betreffenden Faktoren, so kann von einer Unterteilung in quasi 'harte' und 'weiche' standortfaktoren gesprochen werden. Während die 'harten' Standortfaktoren den traditionellen Standortfaktoren entsprechen, zielen die 'weichen' Standortfaktoren verstärkt auf die "Wohn-, Freizeit- und Umweltsituation in einer Region, die Versorgung mit zentralörtlichen Gütern und Dienstleistungen, das Schul- und kulturelle Angebot, die Beratung durch städtische stellen, das Klima usw."68 ab. Unter dem Aspekt des steigenden Le-

$66 \mathrm{Vgl.} \mathrm{Brösse,} \mathrm{U.:} \mathrm{Ziele} \mathrm{in} \mathrm{der} \mathrm{Regionalpolitik} \mathrm{und} \mathrm{in} \mathrm{der}$ Raumordnungspolitik, a.a.0., s. $140 \mathrm{f}$.

67 Siehe exemplarisch Kapitel 3.2.3..

$68 \mathrm{Vgl}$. Eckey, H.-F. U.a.: Sozioökonomische und ökologische Bedingungen für die Entwicklung Nordhessens, a.a.o., s. 157 . 
bensstandards, wird für die zukunft eine stärkere Ausrichtung auf die 'weichen' standortfaktoren erwartet.

Abbildung 2.2: Abgrenzung der Standortfaktoren 69

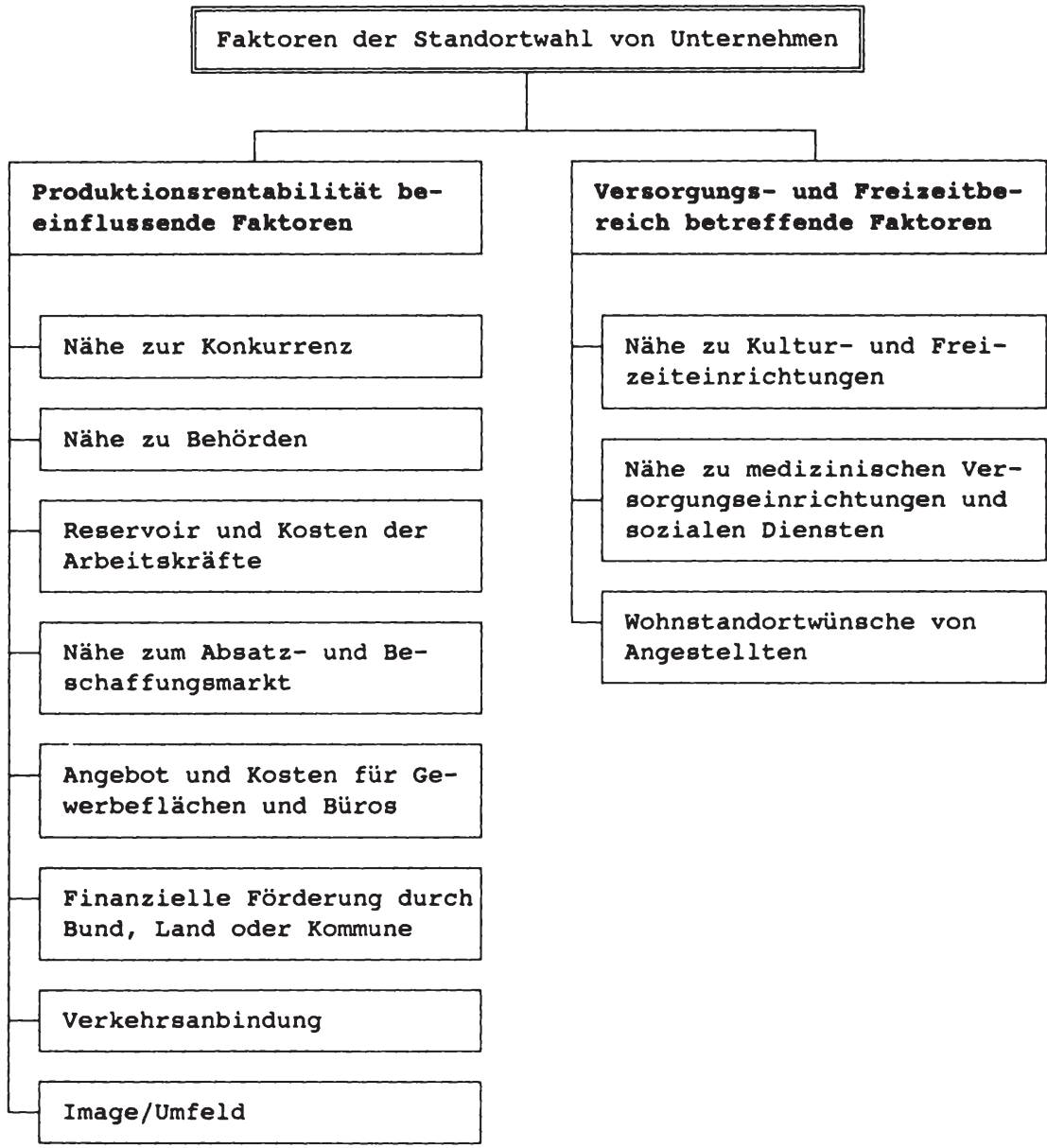

69 In Anlehnung an: Eckey, H.-F. U.a.: Sozioökonomische und ökologische Bedingungen für die Entwicklung Nordhessens, a.a.O., S. 154 . 


\subsubsection{Regionalpolitischer Handlungsbedarf}

Die Leitmaximen zur Gestaltung der Raumstruktur waren in der Vergangenheit durch das wirtschaftswachstum, die Zunahme der Bevölkerungszahl und die siedlungsausweitung geprägt. Sie können zusammengefaßt werden in

- den Ausgleich regionaler Disparitäten bezüglich Lebensbedingungen, Wirtschaftskraft und Entwicklungsmöglichkeiten,

- die gezielte räumliche Verteilung von gewerblichen Arbeitsplätzen,

- den flächendeckenden Ausbau der Verkehrs- und Versorgungsinfrastruktur und

- die Konzentration der siedlungsentwicklung auf zentrale orte. 70

Trotz der in der Vergangenheit vorgenommenen Maßnahmen, um die regionalen Disparitäten zu reduzieren, haben sich die Probleme traditioneller strukturschwacher Räume tendenziell eher vergrößert. 71

Die in der jüngeren Vergangenheit sich verändernden Rahmenbedingungen verlangen somit eine Anpassung der Regionalpolitik an die neuen Gegebenheiten, um das bisher Erreichte zu sichern und zukünftige Fortschritte zu ermöglichen. Die Veränderungen der Rahmenbedingungen umfassen drei zentrale Bereiche: 72

$70 \mathrm{Vgl.} \mathrm{O.V.:} \mathrm{Anforderungen} \mathrm{an} \mathrm{die} \mathrm{Raumordnungspolitik} \mathrm{in}$ der Bundesrepublik Deutschland, in: Raumforschung und Raumordnung, Heft 2/3, 44. Jahrgang, Köln 1986, S. 57.

$71 \mathrm{Vgl}$. Deutscher Bundestag: Programmatische Schwerpunkte der Raumordnung, a.a.0., S. 2 .

$72 \mathrm{Vgl}$. Der Hessische Minister für Wirtschaft und Technik: Nordhessen - Strukturpolitischer Bericht der Hessischen Landesregierung, Wiesbaden 1986, S. 8 ff. 


\section{1. strukturveränderung und stagnation der Bevölkerung}

Die Konkurrenz zwischen den Agglomerationen und den ländlichen Problemregionen, mit ihren traditionell geringen Bevölkerungsdichten, ihrem Mangel an Arbeitsplätzen und ihrer teilweise unterdurchschnittlichen Infrastrukturausstattung, wird durch großräumige wanderungsbewegungen von Norden nach Süden $^{73}$, die gleichzeitig von kleinräumigen wanderungen aus den ländlichen Gebieten in die Randgebiete der zentren überlagert werden, verschärft. 74 Damit verbunden ist eine regional differenzierte demographische Entwicklung.

\section{Technologische Revolution}

Die neuen Technologien werden in zukunft die strukturen des Arbeitsmarktes sowie der gesamten wirtschaft quantitativ und qualitativ tiefgreifend verändern. Der Schaffung neuer Märkte steht die Freisetzung von Kapital und Arbeitskräften in anderen Branchen gegenüber. Diese Entwicklung hat unmittelbaren Einfluß auf die entspechenden Regionen, was anhand der altindustrialisierten Agglomerationen dokumentiert wird, die zu neuen, zusätzlichen Problemgebieten geworden sind.

Verschärft wird diese Entwicklung durch ein 'Technologisches Wettrüsten', das sogar zwischen einzelnen Bundesländern herrscht. 75 Damit verbunden ist eine Konzentration von privatwirtschaftlichen und staatlichen Forschungseinrichtungen sowie hochwertigen Produktionsund Kommunikationstechniken in den Agglomerationen, die

73 Sowohl innerhalb der Bundesrepublik, als auch innerhalb Hessens.

74 Vgl. Wolf, F.: Wirtschaftsschwache Gebiete: Politik der offenen Hand oder Strategie der Staatlich gestützten regionalen Eigenentwicklung?, in: Raumforschung und Raumordnung, Heft 5-6, 45. Jahrgang, Köln 1987, S. 200.

$75 \mathrm{Vgl}$. Der Hessische Minister für Wirtschaft und Technik: Nordhessen - Strukturpolitischer Bericht der Hessischen Landesregierung, a.a.0., S. 8 . 
die Sogwirkung von den ländlich-peripheren Räumen hin zu den Agglomerationen verstärkt.

\section{Umweltkrise}

Der dritte Problemkomplex ist die Krise der natürlichen Umwelt, die objektiv und als Teil des Wertewandels an Bedeutung gewonnen hat. Die Umweltmedien Boden, Wasser und Luft sind wachsenden Belastungen durch Schadstoffe ausgesetzt, welche die Raumnutzungsmöglichkeiten einschränken. Am Beispiel der 'Altlastenproblematik' läßt sich die Diskrepanz früher erzielter ökonomischer Gewinne gegenüber später anfallenden Folgekosten einer Sanierung abschätzen. Um eine wirtschaftliche Expansion auch in Zukunft ermöglichen zu können, müssen die Belastungen der Umwelt drastisch reduziert werden. Eine $\mathrm{Zu}-$ wendung $\mathrm{zu}$ umweltverträglichen Produktions- und Konsumstrukturen ist unumgänglich. 76

Zwischen diesen drei zentralen Bereichen existieren starke Interdependenzen, die bei der Problembewältigung berücksichtigt werden müssen und eine Integration wirtschaftspolitischer Ansätze zu einem koordinierten Gesamtkonzept implizieren. 77

Die o.a. Entwicklungen führen zu veränderten Anforderungen an die Maßnahmen der regionalen Wirtschaftspolitik. Im besonderen Maße gilt dies für die Förderung von Ansiedlungen in strukturschwachen Gebieten, da das seit einem Jahrzehnt bundesweit geschrumpfte gewerbliche Ansiedlungspotential auf ein siebtel zurückgegangen ist. 78 Desweiteren ist die klassische materielle Infrastruktur, die in der Vergangen-

$76 \mathrm{Vgl}$. Deutscher Bundestag: Programmatische Schwerpunkte der Raumordnung, a.a.0., s. $5 \mathrm{f}$.

$77 \mathrm{Vgl}$. Der Hessische Minister für Wirtschaft und Technik, Nordhessen - Strukturpolitischer Bericht der Hessischen Landesregierung, a.a.O., S. $8 \mathrm{ff}$.

78 Ebenda S. 11. 
heit ein ansiedlungsentscheidender standortfaktor war, inzwischen auf einem relativ hohen Niveau angelangt, soda $B$ in diesem Bereich durch weitere Maßnahmen keine entscheidenden Ansiedlungsimpulse mehr erwartet werden. Die Förderung in strukturschwachen Gebieten zielt deshalb primär auf eine steigerung der Angebotsbedingungen und Anpassungsfähigkeit der ansässigen Unternehmen.

Die Bedeutung von Klein- und Mittelbetrieben als Anbieter von Arbeitsplätzen ist in den Vordergrund gerückt. Aus bundesweiten Untersuchungen weiß man, daß Beschäftigungszunahmen größtenteils in der Gruppe der Kleinbetriebe stattfinden. 79 Kleinbetriebe zeigen eine hohe Flexibilität und Innovationskraft, die sie befähigt, auf den strukturwandel $z u$ reagieren. Daraus resultiert, daß Klein- und Mittelbetriebe, stärker als bisher angenommen, für technologische Entwicklungen und wirtschaftliches wachstum verantwortlich sind. Die wirtschaftlichen Erfolge der kleineren und mittleren Unternehmen basieren jedoch zum Teil auf Innovationen, die aus der Grundlagenforschung und Entwicklung neuer Technologien von Großunternehmen resultieren. 80

Für die strukturschwachen Regionen bedeutet dies, daß besondere Anreize für die Schaffung von Arbeitsplätzen in Forschungs- und Entwicklungsbereichen gegeben werden müssen. Dies beinhaltet Bestrebungen, den Mangel an hochwertigen Unternehmensfunktionen und damit hochwertigen Arbeitsplätzen in den Problemregionen abzubauen. Große Bedeutung wird hierbei den Kommunikations- und Informationstechnologien beigemessen.

$79 \mathrm{Vgl}$. Hessisches Ministerium für Wirtschaft und Technik: strukturpolitischer Bericht für Nord- und osthessen, Wiesbaden 1988, S. 6 f.

$80 \mathrm{Vgl}$. Deutscher Bundestag: Raumordnungsbericht 1986, a.a.0., S. 173 . 
Die neuen Informations- und Kommunikationstechnologien beinhalten jedoch auch die Möglichkeit, vorhandene räumliche Trends zu verstärken. Daraus resultiert die Forderung, den Zugang $z u$ den neuen Fernmeldetechniken für stadt und Land gleichermaßen $\mathrm{zu}$ ermöglichen, soweit dies nachfrage- und rentabilitätsorientiert realisierbar ist. 81 Die Entwicklungschancen, welche die neuen Fernmeldetechniken implizieren, müssen auch den problemregionen zugänglich sein, um mögliche Benachteiligungen zu verhindern.

Eine weitergehende Aktivierung der Innovationspotentiale in den strukturschwachen Regionen ist aus regionalpolitischer und aus gesamtwirtschaftlicher Perspektive wünschenswert. Neben den genannten Fördermöglichkeiten zur strukturverbesserung, Ausbau der Infrastruktur, Stärkung der Innovationsfähigkeit, Qualifizierungsmaßnahmen und besondere Unterstützung von Klein- und Mittelbetrieben, stellt die traditionelle regionale Wirtschaftsförderung, in Form von Investitionshilfen für strukturschwache Gebiete, einen Hauptpfeiler der regionalen Wirtschaftspolitik dar.

Unter dem Aspekt einer Harmonisierung der europäischen Wirtschaftspolitik werden die regionalpolitisch begründeten Subventionsprogramme jedoch in zukunft immer stärker eingeschränkt. 82 Hier wird versucht, in Form von Landesförderprogrammen, die Härten des von der Europäischen Gemeinschaft vorgegebenen Rahmens auszugleichen.

Ausgehend von diesen Rahmenbedingungen, muß die regionale wirschaftspolitik nach alternativen Instrumenten suchen, die langfristig die unmittelbaren Finanzierungshilfen für Unternehmen substituieren können.

$81 \mathrm{Vgl}$. Deutscher Bundestag: Programmatische Schwerpunkte der Raumordnung, a.a.0., s. 10.

$82 \mathrm{Vgl}$. Hessisches Ministerium für Wirtschaft und Technik: strukturpolitischer Bericht für Nord- und Osthessen, a.a.0., s. 7 . 


\subsection{Förderungsmöglichkeiten telekommunikativer Techniken}

Die existierenden Förderungsmöglichkeiten telekommunikativer Techniken sind äußerst vielfältig, wobei grundsätzlich zwischen der Förderung der Einführung und Anwendung von telekommunikativen Techniken und den infrastrukturpolitischen Maßnahmen bezüglich der Telekommunikation unterschieden werden kann. Eine überschneidungsfreie zuordnung für alle Förderungsmöglichkeiten ist jedoch nicht möglich.

\subsubsection{Förderung der Einführung und Anwendung}

Die Förderung der Einführung und Anwendung von telekommunikativen Techniken ist im folgenden in die direkte subventionierung, die Informations- und Beratungsinstrumente und in die lokalen und regionalen Telematikvorhaben untergliedert. Die lokalen und regionalen Telematikvorhaben sind zum Großteil Mischformen, die sich zum Teil aus subventionierungen und Informations- und Beratungsleistungen zusammensetzen. Ein Beispiel hierfür stellen die Technologie- und Gründerzentren dar, in denen größtenteils der Mietpreis für die Unternehmen subventioniert ist und zusätzlich Informations- und Beratungsdienstleistungen in unterschiedlichen Ausprägungen angeboten werden. ${ }^{1}$

1 Vgl. Sternberg, R.: Technologie- und Gründerzentren als Instrument kommunaler Wirtschaftsförderung - Bewertung auf der Grundlage von Erhebungen in 31 Zentren und 177 Unternehmen, Dortmund 1988, S. 91. 


\subsubsection{Direkte 8ubventionierung}

Die direkte Subventionierung kann in die Investitionshilfen und in die Subventionierung von Beratungen durch Unternehmensberater untergliedert werden.

\subsection{Investitionshilfen}

Von einer Förderung der Errichtung oder des Ausbaus von Einrichtungen zur Nutzung der neuen Telekommunikationstechniken wurde erstmals im vierzehnten Rahmenplan der Gemeinschaftsaufgabe 'Verbesserung der regionalen wirtschaftsstruktur'2 gesprochen. Für den zeitraum vom 5. Juni 1985 bis zum 31. Dezember 1987 wurde als befristete Probephase der Förderkatalog der Gemeinschaftsaufgabe für den Bereich Telematik geöffnet, wobei die Förderung in jedem Einzelfall der Zustimmung des Unterausschusses der Gemeinschaftsaufgabe bedarf. 3

Grundsätzlich zählen Investitionen im Bereich der modernen Informations- und Kommunikationstechniken $\mathrm{zu}$ den sogenannten 'organisatorischen Innovationen', die von Bund und Ländern in Form von steuerfreien Zulagen und Zuschüssen, zinsvergünstigten Darlehen und Sonderabschreibungen gefördert werden. 4 Voraussetzung für die Förderung ist eine entspre-

2 Vgl. Deutscher Bundestag: Vierzehnter Rahmenplan der Gemeinschaftsaufgabe 'Verbesserung der regionalen wirtschaftsstruktur', Bundestagsdrucksache 10/3562, Bonn 1985 , S. 31 .

$3 \mathrm{Vgl}$. ebenda.

4 Vgl. Richter, H.: Investitionshilfen - Wie man Kommunikationstechnik mit öffentlichen zuschüssen optimal finanziert, in: cogito, Heft 4, 1987, S. 54 . 
chende Zweckbindung, die je nach Förderprogramm unterschiedliche Ausprägungen hat.

Die wichtigsten Förderprogramme des Bundes und der Länder, deren Zweckbindungen und Fördermodalitäten, sind im folgenden überblickartig zusammengestellt: ${ }^{5}$

\section{Förderungen des Bundes}

Die Förderungsprogramme des Bundes umfassen das ERP-Regionalprogramm, das ERP-Existenzgründungsprogramm, die Investitionsförderung der Kreditanstalt für Wiederaufbau, die Bürgschaften der Deutschen Ausgleichsbank und die Projektförderung des Bundesministeriums für Forschung und Technologie. Die einzelnen programme werden im folgenden kurz charakterisiert.

ERP-Regionalprogramm: ${ }^{6}$ AusschlieBlich Klein- und Mittelbetriebe können bei Investitionen zur Errichtung, Erweiterung, grundlegenden Rationalisierung und Umstellung von Betrieben gefördert werden, wenn sie im Fördergebiet liegen und die Investition der 'Verbesserung der regionalen wirtschaftsstruktur' dient. Die Förderung erfolgt in Form zinsgünstiger Kredite bis $z u \quad 300.000$ DM bei einem zinssatz von $8,5 \%$ p.a. im Normalfördergebiet und $7,5 \%$ p.a. im Zonenrandgebiet und einer Laufzeit von bis $\mathrm{zu} 10$ Jahren.

BRP-Existenzgründungsprogramm: ${ }^{7}$ Investitionen zur Errichtung und Einrichtung von Betrieben und damit zusammenhän-

$5 \mathrm{Vgl}$. ebenda S. 54-57.

6 Vgl. Richtlinien für das ERP-Regionalprogramm 1986/87, abgedruckt im Anhang $K$ des 17. Rahmenplans der Gemeinschaftsaufgabe 'Verbesserung der regionalen Wirtschaftsstruktur', a.a.0., S. 204 und Bundesminister für Wirtschaft: Bekanntmachung des Bundesministers für Wirtschaft vom 2.1.1990, in: Bundesanzeiger Nr. 15 vom 23.1.1990.

7 Vgl. Richter, H.: Investitionshilfen - Wie man Kommunikationstechnik mit öffentlichen Zuschüssen optimal finanziert, a.a.0., s. 54 . 
gende Investitionen ${ }^{8}$ können innerhalb der ersten drei Jahre nach Betriebseröffnung gefördert werden. Die Förderung erfolgt in Form zinsgünstiger Kredite bis zu 300.000 DM bei einem zinssatz von $8,5 \%$ p.a. im Normalfördergebiet und $7,5 \%$ p.a. im Zonenrandgebiet und einer Laufzeit von bis zu 10 Jahren.

Investitionsförderung der Rreditanstalt für miederaufbau: ${ }^{9}$ Kleine und mittlere Unternehmen der gewerblichen Wirtschaft können, insbesondere bei Innovationsvorhaben, Rationalisierungsmaßnahmen und Investitionen, gefördert werden, wenn die Investitionen der Errichtung, Sicherung oder Erweiterung des Unternehmens dienen. Die Förderung erfolgt in Form zinsgünstiger Kredite ${ }^{10}$ bis zu 10 Millionen DM bei einem Zinssatz von 8,25 Prozent und einer Laufzeit von bis $\mathrm{zu} 10$ Jahren.

Bürgschaften der Deutschen Ausgleichsbank: 11 Investitionsvorhaben von Freiberuflern und Gewerbetreibenden können bis zu drei Jahre nach Existenzgründung gefördert werden. Die Förderung erfolgt u.a. in Form von Bürgschaften für Bankkredite. 12

8 Insbesondere Investitionen im Bereich der Bürokommunikation.

$9 \mathrm{Vgl}$. Kreditanstalt für Wiederaufbau: Zusammenstellung der Kreditprogramme der Kreditanstalt für Wiederaufbau für Unternehmen der gewerblichen wirtschaft, Frankfurt 1990, S. I. 1 .

10 Bis zu zweidrittel des Investitionsbetrags.

11 Vgl. Bundesminister für Wirtschaft (Hrsg.): Der Mittelstand - Förderungsmaßnahmen des Bundes für mittelständische Unternehmen, Freie Berufe und Existenzgründer, Bonn 1989 , S. 54 .

12 Vgl. Richter, H.: Investitionshilfen - Wie man Kommunikationstechnik mit öffentlichen Zuschüssen optimal finanziert, a.a.0., s. 55 . 
Projektförderung des Bundesministeriums für Forschung und Technologie: 13 Das Bundesministerium für Forschung und Technologie unterstützt die Förderung der raschen Nutzung neuer Technologien durch indirekt-spezifische Maßnahmen und fachprogrammbezogene Projektförderungen für kleine und mittlere Unternehmen. Förderungswürdige Projekte müssen jedoch im Zusammenhang mit Neuentwicklungen stehen. Die Förderung erfolgt je nach Fachprogramm unterschiedlich. Fördermaßnahmen für Klein- und Mittelbetriebe in 1987 waren beispielsweise die Forschungspersonal-Zuwachsförderung, der Personalkostenzuschuß und die Auftragsforschung. 14

\section{Förderungen der Länder}

Aufgrund der Vielfalt der Förderprogramme der einzelnen Länder, werden im folgenden nur die wichtigsten programme, die Investitionen in Büro- und Kommunikationstechnik berücksichtigen, nach Ländern geordnet, dargestellt. Ein Rechtsanspruch auf Förderung besteht bei dem von Bund und Ländern gemeinsam finanzierten Gemeinschaftsprogramm 'Verbesserung der regionalen Wirtschaftsstruktur', das auf die Fördergebiete beschränkt ist. ${ }^{15}$ Bei den Länderprogrammen wird aufgrund der Jahres-Etat-Begrenzung meistens das sogenannte 'Windhund-Verfahren'16 angewandt.

Baden-Württemberg

Programm zur Förderung des Einsatzes moderner Technologien in der mittelständigen wirtschaft: ${ }^{17}$ Die Förderung erfolgt

$13 \mathrm{Vgl.} \mathrm{Bundesminister} \mathrm{für} \mathrm{Forschung} \mathrm{und} \mathrm{Technik:} \mathrm{Bundesbe-}$ richt Forschung 1988, Bonn 1988, S. 106.

$14 \mathrm{Vgl}$. ebenda S. $103 \mathrm{ff}$.

15 Vgl. Kapitel 2.1.1.3. Rechtliche Grundlagen.

16 Die Anträge werden nach Antragseingang bearbeitet und bis zum Erreichen der Etatgrenze genehmigt.

$17 \mathrm{Vgl}$. Ministerium für Wirtschaft, Mittelstand und Technologie des Landes Baden-Württemberg (Hrsg.): Wirtschaftsförderungsprogramm - Technologieförderung, stuttgart 
in Form zinsgünstiger Kredite bis zu 70 Prozent der Investitionssumme bei einer Laufzeit von bis zu 15 Jahren und durch Bürgschaften bis $z u$ einer Höhe von einer Millionen DM. In bestimmten Fällen können Zuschüsse bis zu $15 \%$ der Anschaffungskosten gewährt werden. Für Entwicklungsvorhaben im Bereich moderner Technologien können Lohn- bzw. Entwicklungskosten bis $z u$ einer Höhe von 70 Prozent ${ }^{18}$ bezuschußt werden.

zuwendungen für Existenggrüdung und Existensfestigung: ${ }^{19}$ Investitionen in moderne Kommunikationstechniken, die zur 'Existenzfestigung' dienen, können bei Gewerbetreibenden und Freiberuflern gefördert werden. Die Förderung erfolgt in Form zinsgunstiger Kredite bis zu einer Höhe von 300000 DM und einer Laufzeit von bis zu 20 Jahren und durch Bürgschaften bis zu einer Höhe von einer Millionen DM.

\section{Bayern}

Bayerisches Kittelstands-Kreditprogramm: 20 Investitionen in den Bereichen Einrichtung, Rationalisierung und Modernisierung von Betrieben, sowie die Anwendung neuer Technologien können durch zinsgünstige Kredite bis zu 25 Prozent des Investitionsbetrages gefördert werden. Der zinssatz beträgt 6,5 Prozent im Normalfördergebiet und 5,5 Prozent im zonenrandgebiet bei einer Laufzeit von bis zu 15 Jahren.

1989, S. 3-10.

18 Nur in Ausnahmefällen.

$19 \mathrm{Vgl.} \mathrm{Ministerium} \mathrm{für} \mathrm{Wirtschaft,} \mathrm{Mittelstand} \mathrm{und} \mathrm{Techno-}$ logie des Landes Baden-Württemberg (Hrsg.): Wirtschaftsförderungsprogramm - Existenzgründung - Existenzfestigung - Technolgieorientierte Unternehmensgründung, Stuttgart 1987, S. 5-13.

$20 \mathrm{Vgl}$. Bayerisches Staatsministerium für wirtschaft und Verkehr: Bayerische Finanzierungshilfen für den gewerblichen Mittelstand, München 1989, S. 2-3. 
Bayerisches regionales Förderprogramm: 21 Die Einführung neuer Technologien kann in Unternehmen mit regionalwirtschaftlicher Bedeutung gefördert werden. Voraussetzung ist, da $B$ die Investitionen der Schaffung oder sicherung von Arbeitsplätzen dienen. Die Förderung erfolgt durch zinsgünstige Kredite bis zu 50 Prozent der Investitionssumme. Der Zinssatz beträgt 4,5 Prozent bei Neuansiedlungen und 6 Prozent für sonstige Vorhaben bei einer Laufzeit von 8 bis 15 Jahren.

Berlin

Das Berlinfördergesetz ermöglicht der stadt, steuerfreie Investitionszulagen $\mathbf{u} . \mathbf{a}$. in Höhe von 25 Prozent der Investitionskosten zu gewähren, die auch für den Bereich der Informations- und Kommunikationstechnik gelten.

Weitere relevante Förderungen in Berlin sind der sonderfonds des senats zur Förderung kleiner und mittlerer onternehmen, der Innovationsfonds und die ERP-Darlehen zur Förderung von Investitionen in Berlin.

\section{Bremen}

Investitionszuschüsse an kleine und mittlere onternehmen: Investitionen können mit projektbezogenen und nicht rückzahlbaren Zuschüssen bis zu 7,5 Prozent einer 'betriebswirtschaftlich sinnvollen' Investitionssumme gefördert werden. Zusätzlich können weitere Investitionszulagen bis zu 15 Prozent der Investitionssumme beantragt werden, wenn eine 'Verbesserung der regionalen Wirtschaftsstruktur' $22 \mathrm{zu}$ erwarten ist.

\section{Hamburg}

Mittelstandsförderungsprogramm: Investitionen für Betriebseinrichtungen oder zur schaffung von Arbeits- und Ausbil-

21 Vgl. ebenda S. 3-4.

22 Z.B. durch Betriebserrichtung oder -erweiterung. 
dungsplätzen können mit Investitionszuschủssen bis zu 100.000 DM gefördert werden. Zusätzlich kann bei Neugründungen innerhalb der ersten drei Jahre das Existenzgründungsprogramm in Anspruch genommen werden, das Investitionszuschüsse bis zu 200000 DM vorsieht.

\section{Hessen}

Frankfurter Innovations-Förderung für kleine und mittlere Unternehmen: Im Stadtgebiet Frankfurt können Investitionen gefördert werden, die den Einsatz neuer Technolgien fördern und die Adaption an moderne Entwicklungen erleichtern. Die Förderung erfolgt durch Beratungszuschüsse bis zu 75 Prozent und durch subventionierte Kredite mit Laufzeiten von bis zu 10 Jahren.

HLT-8onderkreditprogramm: ${ }^{23}$ Bestimmte Investitionen im gewerblichen Bereich können regional begrenzt gefördert werden. 24 Die Förderung erfolgt in Form zinsgünstiger Kredite der Hessichen Landesentwicklungs- und Treuhandgesellschaft $\mathrm{mbH}$ (HLT) bei einer Laufzeit von 10 Jahren. Die Kredite sind auf maximal 400000 DM begrenzt und können in Höhe von bis zu 50 Prozent der Investitionssumme gewährt werden.

\section{Niedersachsen}

Technologieprogramm: 25 Innovationsvorhaben im Rahmen von Kooperationen von kleinen und mittleren Unternehmen können auf der Basis einer Projektförderungen mit zuschüssen bis

$23 \mathrm{Vgl}$. Hessisches Ministerium für Wirtschaft und Technik: Strukturpolitischer Bericht für Mittelhessen - Beiträge zur Wirtschaftspolitik in Hessen, Heft 16, Wiesbaden 1988 , S. 59 .

24 Dies sind strukturschwache Gebiete außerhalb der Fördergebiete der Gemeinschaftsaufgabe zur 'Verbesserung der regionalen Wirtschaftsstruktur'.

25 Vgl. Niedersächsisches Ministerium für Wirtschaft, Technologie und Verkehr: Förderung von Forschungs- und Entwicklungsvorhaben auf dem Gebiet der Produkt- und Verfahrensinnovation, Hannover $1989,5 / 111$. 
zu 75 Prozent der Investitionen gefördert werden. Innovationsvorhaben können bis zu 50 prozent bezuschußt werden, in der Regel jedoch nicht höher als 300000 DM. Weiterhin sind Beratungen im Bereich der 'elektronischen Datenverarbeitung' zuschußfähig.

Nordrhein-Westfalen

Technologie-Programm wirtschaft: 26 Investitionen und Neugründungen kleiner und mittlerer Unternehmen im Bereich neuer Technologien können gefördert werden, indem die technische Beratung mit bis zu 50 prozent der Kosten bezuschußt werden kann. Einzelprojekte, die einen 'gesamtwirtschaftlichen Nutzen' erwarten lassen, können zusätzlich mit Personal- und Sachkostenzuschüssen bis zu 65 prozent gefördert werden. 27

Regionales Wirschaftsförderungsprogramm: 28 Investitionen im Bereich Datenverarbeitung (inclusive Datenbanken) können mit Investitionszuschüssen bis $z u \quad 20$ prozent und zinsgünstigen Darlehen unterstützt werden.

Rheinland-Pfalz

Von diesem Bundesland wird kein spezielles Programm im Bereich der Technologieförderung angeboten. Investitionen im Bereich Informations- und Kommunikationstechniken können jedoch mit dem zinszuschußprogramm des Landes gefördert werden, das u.a. Rationalisierungs- und Modernisierungsin-

26 Vgl. Minister für Wirtschaft, Mittelstand und Technologie des Landes Nordfhein-Westfalen: Wirschaftspolitik für Nordrhein-Westfalen -Zwischenbilanz des MWMT-, Düsseldorf 1987, S. 22 ff.

$27 \mathrm{Vgl}$. Minister für Wirtschaft, Mittelstand und Technologie des Landes Nordfhein-Westfalen: TelekommunikationsProdukte und -Dienstleistungen, Chancen für Hersteller und Anbieter in NRW, Düsseldorf 1988, S. 50.

$28 \mathrm{Vgl}$. Minister für Wirtschaft, Mittelstand und Technologie des Landes Nordfhein-Westfalen: NRW Technologieprogramm Wirtschaft und Programm Zukunftstechnologien, Düsseldorf 1985, S. 1-4. 
vestitionen mittelständiger Betriebe unterstützt. Die Förderung erfolgt durch Kreditzuschüsse in Höhe von 3,5 Prozent p.a.. Regional beschränkt werden zusätzlich Rationalisierungs- und Erweiterungsinvestitionen bezuschußt.

\section{Saarland}

Mittelstands-Förderungsprogramm: Investitionen von Gewerbetreibenden und Freiberuflern, die der Errichtung, Erweiterung, Modernisierung, Rationalisierung oder Umstellung ihrer Betriebe dienen, können gefördert werden. Die Förderung erfolgt durch $\mathrm{zinsgünstige} \mathrm{Kredite} \mathrm{bis} \mathrm{zu}$ einer Höhe von einer Millionen DM und einer Laufzeit von bis zu 15 Jahren.

Schleswig-Holstein

Mittelstands-strukturprogramm: Investitionen von Gewerbetreibenden und Freiberuflern werden durch zinsgünstige Kredite bis maximal 600000 DM gefördert. Der Zinszuschuß beträgt 3 Prozent. Bei Existenzgründungen können Betriebsmittelkredite mit zinszuschüssen zwischen 4 und 6 prozent und zusätzlich Investitionszuschüsse, bei 'betriebsnotwendigen' Aufwendungen, bis zu 15 prozent gewährt werden. Weitere Subventionen können für Betriebsberatungen bis zu einer Höhe von 60 Prozent der Kosten gewährt werden.

\subsection{Subventionierung von individuellen Beratungen}

Subventionen für individuelle Beratungen können für den Bereich organisatorische und technische Fragen der Betriebsführung in Anspruch genommen werden, wenn die Beratung einer steigerung der Wettbewerbsfähigkeit des Unternehmens dient. 29 In diesen Bereich fallen auch Beratungen im Zusam-

29 Vgl. Bundesminister für Wirtschaft: Richtlinien des Bundesministers für Wirtschaft über die Förderung von Unternehmensberatungen für kleine und mittlere Unternehmen vom 20. November 1989, Bonn 1989. 
menhang mit der Kommunikationstechnik. Die Förderung erfolgt in Form eines Zuschusses, der für eine allgemeine Beratung 50 Prozent der berechneten Beraterhonorare und spesen beträgt und auf maximal 3000 DM beschränkt ist. Bei Existenzgründungen und bis zu zwei Jahren danach beträgt der Zuschuß 60 Prozent der Beratungskosten bis maximal 2.500 bzw. 3.000 DM. 30

Voraussetzung, um einen Antrag auf Förderung stellen $z u$ können, ist erstens die bezahlte Beratungsrechnung und zweitens ein qualifizierter Bericht des beauftragten Unternehmensberaters. Die Anträge selbst werden über verschiedene Leitstellen abgewickelt, wie z.B. den Deutschen Industrie- und Handelstag, die Gewerbeförderungsgesellschaft des Bundesverbandes der Selbständigen und den Zentralverband des Deutschen Handwerks.

Speziell für Kleinbetriebe vermittelt das Rationalisierungs-Kuratiorium der Deutschen Wirtschaft in Eschborn, das von Bund und Ländern gefördert wird, Betriebsberatungen, die in Höhe von 40 prozent des Beratungshonorares bezuschußt werden. 31

Grundvoraussetzung für eine Förderung durch die angeführten Förderprogramme ist jedoch, daß die projekte bereits im Vorstadium die Gewähr für eine 'wirtschaftlich sinnvolle' Investition bieten.

$30 \mathrm{Vgl}$. ebenda.

31 Vgl. Richter, H.: Investitionshilfen - Wie man Kommunikationstechnik mit öffentlichen zuschüssen optimal finanziert, a.a.o., s. 57 . 


\subsubsection{Informations- und Beratungskonzepte}

Informations- und Beratungsaufgaben bezüglich der neuen telekommunikativen Techniken werden von verschiedenen Institutionen wahrgenommen. Im folgenden werden einige Konzepte zur Förderung der Anwendung von Telekommunikation dargestellt. Es handelt sich hierbei nicht um einen vollständigen und ausgewogenen Überblick, da die Entwicklungen in diesem Bereich außerordentlich schnell vorangehen und Informationen über neue Initiativen teilweise nur unzureichend vorhanden sind.

\subsection{Information und Beratung durch die Deutsche Bundespost}

Die Deutsche Bundespost als Betreiber der öffentlichen Netze stellt die notwendige Infrastruktur zur Verfügung und bietet die Dienste und Dienstleistungen zur Nutzung dieser öffentlichen Netze an.

Im Rahmen des Marketing der angebotenen Netze und Dienste, werden kundengerechte Informationen angeboten und Präsentationen der Anwendungsmöglichkeiten der einzelnen Dienste vorgenommen, bei denen besonders die mit der Anwendung verbundenen wirtschaftlichen Vorteile im Vordergrund stehen.

Neben den Informationsangeboten der Deutschen Bundespost, in Form von Broschüren, wie beispielsweise 'Das ISDN-Angebot der Deutschen Bundespost'32, werden kostenlose ISDN-Anwenderseminare ${ }^{33}$ im Bereich ISDN, BTX, TELEBOX u.a. durch-

32 Bundesministerium für das Post- und Fernmeldewesen (Hrsg.): Das ISDN-Angebot der Deutschen Bundespost, Bonn 1988 .

$33 \mathrm{Vgl}$. Deutsche Bundespost: Post-Seminare 1989, Frankfurt 1989. 
geführt. Speziell für die junge Generation sowie kleine und mittlere Betriebe sind für Hessen in den Orten Heusenstamm, Wiesbaden und Kassel Informationszentren eröffnet worden, um die zielgruppe an die Telekommunikation heranzuführen.

Für betriebsspezifische Anwendungsprobleme bietet die Deutsche Bundespost eine spezielle Beratung vor ort durch Technische Vertriebsberater an, die individuelle Lösungen für den entsprechenden Betrieb ausarbeiten. 34

\subsection{Innovations- und Technologieberatungsstellen}

Seit 1976 werden von der Bundesregierung Pilotprojekte zur Forschungs- und Technologieförderung in Form von Innovationsberatungs- und Technologietransferstellen gefördert. 35 Hauptträger sind die Industrie- und Handelskammern, die Handwerkskammern und das Rationalisierungskuratorium der deutschen Wirtschaft. Von den technologischen und ökonomischen Beratungsdiensten profitieren vorrangig kleine und mittlere Unternehmen, die im besonderen auf Hilfestellungen und Beratungen angewiesen sind, um ihre Wettbewerbs- und Leistungsfähigkeit $\mathrm{zu}$ erhalten und $\mathrm{zu}$ steigern. ${ }^{36}$ Dies gilt vor allem für Unternehmen im ländlich geprägten Raum.

\section{Innovations - und Technologieberatung}

Die Innovations- und Technologie-Beratungsstelle Hessen beispielsweise stellt eine Gemeinschaftseinrichtung der zwölf hessischen Industrie- und Handelskammern dar. Sie hat

34 Vgl. Hessicher Minister für Wirtschaft und Technik: Beiträge zur Wirtschaftspoltik in Hessen Heft 13 - Nutzung der Informations- und Kommunikationstechnik in Hessen, Wiesbaden 1987 , S. 26 f.

$35 \mathrm{Vgl}$. Deutscher Bundestag: Raumordnungsbericht 1986 der Bundesregierung, a.a.0., S. 77 .

$36 \mathrm{Vgl}$. ebenda. 
das Ziel, mit Hilfe gezielter Beratungen und Informationen über den stand der Technik und deren Anwendung, die Wettbewerbsfähigkeit der hessischen Betriebe und die Stabilisierung der Wirtschaftskraft der Unternehmen zu fördern. 37

Das Leistungsangebot der Innovations- und Technologie-Beratungsstelle umfaßt die Unterstützung bei Rontakten zur Wissenschaft, Verwaltung und wirtschaft, die Information über Technologietrends, Technologietransfer, Förderungsprogramme und Veranstaltungen und die Beratung in Form von Problemdiagnosen, Lösungsvorschlägen, Leistungsvermittlungen, Kontaktvermittlungen und Literaturrecherchen. 38 Neben diesem Leistungsangebot besteht zusätzlich eine Informationsberatung über die Nutzungsmöglichkeiten von Datenbanken. 39 Sämtliche Beratungen durch die Innovations- und Technologie-Beratungsstelle der hessischen Industrie- und Handelskammern sind kostenfrei.

\section{Technologietransfer}

Technologietransfer stellt eine konkrete Kooperation bei der Vermittlung, Entwicklung und wirtschaftlichen Nutzung von neuen Produkten und Verfahren zwischen Hochschulen und Unternehmen dar. 40 Die Kooperationen finden beispielhaft in folgenden Bereichen statt:

$37 \mathrm{Vgl}$. Innovations- und Technologie-Beratungsstelle Hessen: Technologieberatung - Hilfe bei Forschung und Entwicklung in Hessen, Frankfurt o.J..

$38 \mathrm{Vgl}$. ebenda.

$39 \mathrm{Vgl}$. Innovations- und Technologie-Beratungsstelle der hessischen Industrie- und Handelskammern: Informationen aus Datenbanken - Beschaffung und Beratung, Frankfurt 1989 .

$40 \mathrm{Vgl}$. Allesch, J.: Regionale Entwicklung durch Technologietransfer, Innovation und Qualifikation - das Berliner Modell, in: Frankfurter Allgemeine Zeitung Nr. 131/1986 vom 10.Juni 1986, Beilage 'Berater und Dienstleister', S. B15. 
- Beratung und Hilfe bei Unternehmensgründungen und bei der Konzeptions- und Entwicklungsphase von Produkt- und Verfahrensinnovationen,

- Inanspruchnahme des Forschungspotentials der Hochschulen durch die wirtschaft, 41

- Technological Forecast-Foren in den Unternehmen sowie

- Personaltransfer zwischen Wissenschaft und Wirtschaft. 42

Technologietransferstellen wurden an Hochschulen und Fachhochschulen geschaffen, um den Technologie- und Wissenstransfer zu fördern. Die Transferstellen haben in der Regel die Aufgabe

- ailgemeine Auskünfte über Leistungsmöglichkeiten zu erteilen,

- fachliche Kontakte zu Professorinnen und Professoren $z u$ vermitteln,

- bei der Projektdefinition und -finanzierung unterstützend tätig zu werden,

- Projekte in Absprache mit Trägern und Durchführenden organisatorisch vom Angebot bis zur Abrechnung abzuwickeln,

- den Personaltransfer zwischen Hochschule und ihren Kooperanden zu unterstützen und

- die interessierte öffentlichkeit in geeigneter Weise über das Technologie- und Wissenstransferangebot $\mathrm{zu}$ informieren. 43

Rechtlich selbständige Institutionen, wie beispielsweise das Institut für Wirtschaftsinformatik der Universität

41 Beispielsweise in Form von Auftragsforschung.

42 Vgl. Rürup, B.: Wirtschaftliche und gesellschaftliche Perspektiven der Bundesrepublik Deutschland, München 1989 , S. 86.

$43 \mathrm{Vgl}$. Wirtschaftsjunioren der Industrie- und Handelskammer Darmstadt mit Unterstützung der stadt Darmstadt (Hrsg.): Handbuch für Technologietransfer, Darmstadt 1989, S. 10. 
Frankfurt, können außerhalb der Universitätsverwaltung Verträge mit Mitarbeitern und Kooperationspartnern abschliessen, was eine engere Verbindung zwischen Forschung und Lehre ermöglichen soll. 44

Zusätzlich besteht in einigen Ländern die Einrichtung von sogenannten Kontaktdozenten an Fachhochschulen, die technische und wirtschaftliche Beratungen durchführen. 45

\subsubsection{Lokale und regionale Telematikvorhaben}

\subsection{Teleport und Telehaus}

\section{Teleport}

In Deutschland wurde die Teleport-Idee 1983 bekannt. 46 'Teleport' ist ein Modewort, mit dem unterschiedliche regionale Projekte und Initiativen bezeichnet werden, ohne daß eine eindeutige Definition zugrundeliegt. 47 Für die verschiedenen lokalen Initiativen wurden teilweise unterschiedliche Begriffe wie Teleport, Telecenter, Mediapark, Technoport und Teletopia verwendet. ${ }^{48}$ Die deutsche Interpretation des Begriffs Teleport, "als programmatische Be-

44 Vgl. Hessicher Minister für Wirtschaft und Technik: Beiträge zur Wirtschaftspoltik in Hessen Heft 13 - Nutzung der Informations- und Kommunikationstechnik in Hessen, a.a.o., s. 28 .

$45 \mathrm{Vgl}$. Deutscher Bundestag: Raumordnungsbericht 1986 der Bundesregierung, a.a.0., s. 77 .

$46 \mathrm{Vgl}$. Lange, S. u.a.: Telematik und regionale Wirtschaftspolitik, a.a.0., s. B-17.

47 Vgl. Schnöring, T.: Wirkungen der Telekommunikationsentwicklung auf Anwender, a.a.0., S. 51.

$48 \mathrm{Vgl}$. Lange, S.: Teleports - Entstehung und Förderung der Telematik, in: Innovation und Management, Heft 3, 1986, S. 287 . 
zeichung für unterschiedliche lokale Vorhaben, in denen kommunikationstechnische Infrastruktur für lokale und weltweite Schmal- und Breitbandkommunikation und wirtschaftliche Entwicklung miteinander verbunden werden"49, scheint sich durchzusetzen. Die Begriffsbildung ist jedoch noch nicht abgeschlossen.

Zur Förderung des Austausches von Informationen zwischen den Initiativen, entstand 1984 die World Teleport Association (WTA). Bisher haben vier Welt-Teleport-Kongresse stattgefunden. Die Welt-Teleport-Konferenzen in Tokio 1985, in Amsterdam 1986 und in San Franzisco 1987 haben sich primär mit den Planungen der Teleports beschäftigt. 50 Auf der vierten Welt-Teleport-Konferenz in Köln 1988 standen erste Erfahrungsberichte an. Themen der Arbeitsgruppen waren

- stadtplanerische und regionale Aspekte der Teleports,

- technische Lösungen für die interne Kommunikation von Teleports,

- Teleports als Dienstleistungszentren für elektronische Medien,

- Teleports als Dienstleistungszentren für die Geschäftskommunikation sowie

- Telekommunikation zwischen Deregulierung und Nutzerinteressen. 51

Mit Beginn der Werbung für den Teleport New York hat die Idee in der Telematik ein neues Instrument der lokalen

49 Lange, S.: Teleports: Neue Aufgaben für die kommunale Wirtschaftspolitik, in: ONLINE 87, 10. Europäische Kongreßmesse für Technische Kommunikation, Hamburg 1987, S. 1.7.1.08.

$50 \mathrm{Vgl}$. Lange, S.: Neue Aufgaben für die kommunale Wirtschaftspolitik, in: Akademie für Raumforschung und Landesplanung (Hrsg.): Räumliche Wirkungen der Telematik, Hannover 1987, S. 510.

$51 \mathrm{Vgl}$. Lange, S.: Lokales Potential für den Weltmarkt, in: net, 42 , Heft 9,1988, S. 334 . 
Wirtschaftspolitik zu sehen, weltweit Anhänger gefunden. Lokale Initiativen gibt es im Ausland neben New York, beispielsweise in Yokohama, London und Amsterdam und in der Bundesrepublik in Bremen, Köln und Karlsruhe. Die Initiativen werden von der privaten Wirtschaft, den Kammern, den städten, den Infrastrukturbehörden und den Postverwaltungen getragen. 52

Auslösender Faktor für die Entstehung der amerikanischen Teleports sind die lokalen Defizite der kommunikationstechnologischen Infrastruktur und das Fehlen eines flächendekkenden Angebotes der Dienste zu einheitlichen Gebühren. 53 Die Infrastruktur der Telematik stellt somit in den Vereinigten staaten einen Standortfaktor dar, der Unternehmensentscheidungen beeinflussen kann.

Die Deutsche Bundespost bietet im Vergleich zum Ausland neue Fernmeldedienste relativ schnell flächendeckend und $z u$ gleichen Bedingungen an. Solang dies gewährleistet ist, können sich die lokalen Initiativen in der Bundesrepublik Deutschland verstärkt auf die Anwendungsförderung konzentrieren. Im Vergleich $z u$ den USA besteht in der Anwendung der angebotenen Fernmeldedienste und der Entwicklung kundenorientierter Angebote das größte Defizit. 54 "Der interregionale Wettbewerb in Deutschland in bezug auf die Telematik entsteht also nicht, wie in den USA, aufgrund von regionalen Unterschieden in der kommunikationstechnischen Infrastruktur, sondern in bezug auf neue Dienstleistungen, die die Telematik für die Wirtschaft nutzbar machen."55

52 Vgl. Lange, S.: Teleports - Entstehung und Förderung der Telematik, in: Innovation und Management, a.a.o., S. 285 .

53 Vgl. ebenda S. 285.

$54 \mathrm{Vgl}$. Lange, S.: Neue Aufgaben für die kommunale Wirtschaftspolitik, in: Akademie für Raumforschung und Landesplanung (Hrsg.): Räumliche Wirkungen der Telematik, a.a.0., s. 508 .

55 Ebenda S. 509 . 
Teleport-Vorhaben mit jeweils unterschiedlichen Ausprägungen bestehen in der Bundesrepublik Deutschland in Köln, Bremen und Karlsruhe. 56

Mit dem Konzept des 'MediaParks' soll in Köln die Idee einer Verbindung von stadtplanung und Telekommunikation realisiert werden. "Der Mediapark als neuer Stadtteil soll Firmen und organisationen aus den Bereichen neue Medien, Kunst und Kultur, Aus- und Weiterbildung, Forschung und Entwicklung einen attraktiven standort bieten, der mit moderner Kommunikationsinfrastruktur ausgestattet wird und über kurze Wege die persönliche Kommunikation zwischen den Fachleuten erlaubt."57 Folgende Nutzungen sollen im Medienpark gebündelt realisiert werden:

- die Medienproduktion,

- die geschäftliche Kommunikation und Informationsverarbeitung,

- die Präsentation der Möglichkeiten der Telekommunikation und Informationsverarbeitung,

- die Forschung und Ausbildung im Bereich der Informationsund Kommunikationstechniken und

- die dazugehörigen Dienstleistungen. 58

Bremen bietet eine andere Interpretation von Teleport. Die Verbindung von wirtschaftlicher Entwicklung und Technik dominiert gegenüber der stadtplanung. Der Produktionsfaktor 'Information und Kommunikation' soll gezielt verstärkt werden und die Standortqualität der Hansestadt Bremen erhöhen. Dafür wurde ein spezielles wirtschaftsstrukturpolitisches

$56 \mathrm{Vgl}$. Lange, S.: Lokales Potential für den Weltmarkt, a.a.o., S. 338 .

57 Ebenda.

$58 \mathrm{Vgl}$. Georgieff, P. u.a.: Lokale und regionale TelematikVorhaben in der Bundesrepublik Deutschland, FraunhoferInstitut für systemtechnik und Innovationsforschung (Hrsg.), Karlsruhe 1987, S. 44. 
Aktionsprogramm 59 geschaffen, daß bis 1995 innovative und zukunftsweisende Vorhaben der Telematik fördert. 60 Neben der regionalen Förderung sollen neuartige Telekommunikations-Dienstleistungen entstehen, die sowohl regional als auch bundes- oder weltweit angeboten werden.

Ein Beispiel hierfür ist das im Juni 1989 eröffnete Telekommunikationslabor der Bremer Universität. Zielsetzung des Labors ist die Analyse von Anwendungen und Möglichkeiten der vorhandenen Dienste, um Leitsätze für die Auswahl maßgeschneiderter Telekommunikationslösungen für Klein- und Mittelbetriebe $\mathrm{zu}$ erarbeiten. 61 Das mit Anschlüssen und Endgeräten ausgestattete Labor wird von Bremen drei Jahre finanziert, anschließend sollen die entwickelten Beratungskonzepte von Innovations- und Unternehmensberatern und den Handwerks- und Handelskammern genutzt werden. 62

Ein weiteres Beispiel ist das eigentliche Bremer TeleportProjekt. 63 Der Teleport-Bremen soll der Seehafenverkehrswirtschaft, den Handelshäusern, den Banken und Versicherungen als Dienstleistungszentrum dienen, indem Daten und Informationen über ein Kommunikationszentrum weltweit versendet und empfangen werden. Das Fraunhofer-Institut für systemtechnik und Innovationsforschung hat die Rolle des organisators, stifters von Kooperationen, Ideenlieferanten, Beraters für Unternehmen und Behörden, Informationsbeschaffers und Evaluators übernommen. Unterstützt wird das Insti-

59 Siehe auch Kapitel 2.3.1.1.1.

$60 \mathrm{Vgl}$. Lange, S.: Lokales Potential für den Weltmarkt, a.a.o., S. 335 .

61 Vgl. o.V.: Telekommunikationslabor soll kleine und mittlere Unternehmen beraten, in: VDI-Nachrichten, Bremen 30.06 .89 .

$62 \mathrm{Vgl}$. ebenda.

$63 \mathrm{Vgl}$. Lange, S.: Lokales Potential für den Weltmarkt, a.a.o., S. 335 . 
tut zusätzlich durch einen 'Telematik-Promotor', der vor ort für die Umsetzung der Ziele des Förderprogramms zuständig ist. 64

Rarlsruhe interpretiert Teleport im sinne von 'stadtentwicklung und Telematik'. Obwohl in Karlsruhe der Begriff Teleport als solcher nicht verwendet wird, kann die summe der Vorhaben in Karlsruhe doch als Teleport bezeichnet werden. 65

Unter dem Namen 'Zentrum für Informations- und Kommunikationstechnologien' (ZIK) wurde 1988 der Teleport in Karlsruhe, als Tochter des IHK-Bildungszentrums eröffnet. Das Zentrum soll Chancen und Risiken technisch-wirtschaftlicher Entwicklungen aufzeigen und die notwendigen Anwendungsprozesse fördern. Weiterhin wird der Ausbau von Informationsund Beratungslücken sowie die Beseitigung von Anwendungshemmissen angestrebt. 66

In Köln, Bremen und Karlsruhe entstehen mit den Teleports neue Instrumente einer lokalen wirtschaftsförderung, die über den eigentlichen Teleport-Ansatz hinausgehen. Neben den Großstädten ist diese Entwicklung auch in kleineren Städten wie beispielsweise in Nordhorn, in Niedersachsen an der Grenze zu Holland festzustellen, wo das 'Telehaus Nordhorn' 1988 in Betrieb genommen wurde. 67

64 Vgl. ebenda.

$65 \mathrm{Vgl}$. Lange, S.: Teleports - Entstehung und Förderung der Telematik, in: Innovation und Management, a.a.o., S. 287 .

$66 \mathrm{Vgl}$. Lange, S.: Lokales Potential für den Weltmarkt, a.a.o., S. 338 .

$67 \mathrm{Vgl}$. ebenda. 


\section{Telehaus}

Der Begriff Telehaus wurde 1984 vom Fraunhofer-Institut für Systemtechnik und Innovationsforschung eingeführt. ${ }^{68}$ Die Grundidee stellt das Ausgliedern von innerbetrieblichen Aufgaben mehrerer Unternehmen dar, die von einer zentralen Stelle ausgeführt werden sollen. Grundsätzlich bieten sich drei organisationsformen an:

- das originäre Telehaus (= Ansiedlung von mehreren Unternehmen in einem Gebäude),

- das kooperative Telehaus (= Gründung einer Unternehmung durch mehrere Kooperationspartner, die gleichzeitig Teilhaber des Unternehmens sind) und

- das konvertierte Telehaus (= Neugründung eines Unternehmens, das als Dienstleistungsbetrieb die Aufgaben der Kunden erledigt). 69

Das Telehaus in Nordhorn ist in einem Anbau des Technologie- und Gründerparks Nordhorn untergebracht. In den ersten fünf Jahren wird das Telehaus als eigenständige Abteilung der Betriebsgesellschaft 'Innovation-Technologie-Nordhorn GmbH' geführt, die auch den Technologie- und Gründerpark leitet. Die Finanzierung des Telehauses in Höhe von voraussichtlich 1,035 Millionen DM, soll mit einer Förderung durch die Gemeinschaftsaufgabe (80\%), einer Förderung durch den Landkreis (10\%) und einer Förderung duch die stadt (10\%) gewährleistet werden. 70

Die geplanten Nutzungen und Einrichtungen im Telehaus umfassen:

$68 \mathrm{Vgl}$. Lange, S. U.a.: Lokale Initiativen zur Förderung der Anwendungen der Telekommunikation, a.a.o., S. 147 .

$69 \mathrm{Vgl}$. ebenda.

$70 \mathrm{Vgl}$. Georgieff, P. u.a.: Lokale und regionale TelematikVorhaben in der Bundesrepublik Deutschland, a.a.o., s. 82 . 
- Datenbankrecherchen über Datex-P,

- Videokonferenzen,

- Bildschirmtext und Mailbox,

- Ausstellungen und Werkstatt,

- Schreibdienst mit Teletex sowie

- Büro mit Telefon und Telefax. 71

Die Zielgruppe des Telehauses sind kleine und mittlere Unternehmen im ländlichen Raum. Neben der kostengünstigen gemeinsamen Nutzung der neuen Telekommunikationstechniken werden ergänzende Dienstleistungen wie Information, Schulung und Beratung angeboten, die auf die konkreten Bedürfnisse der Unternehmen zugeschnitten werden. Im Detail sollen folgende Dienstleistungen angeboten werden:

- die Vermittlung von Übersetzungen bei Fachbüros im Inund Ausland,

- die Vermittlung von wirtschaftsauskünften und Durchführung von Datenbankrecherchen,

- die Durchführung von Video- und Telekonferenzen,

- die Vorstellung, Vermittlung und betriebsspezifische Eignungsprüfung von Hard- und Software-Angeboten,

- eine Übungswerkstatt und ein Telefonladen,

- Schulungen zur Anwendung von Informations- und Kommunikationstechniken,

- die Beratung zum Einsatz von Informations- und Kommunikationstechniken für bestimmte Betriebs- bzw. Anwendungsbereiche und

- die Unterstützung und Kontaktvermittlung bei einschlägigen Forschungs- und Entwicklungsarbeiten. 72

Das Telehaus-Projekt im Technologie- und Gründerpark Nordhorn zeichnet sich einerseits durch eine grenzüberschreitende orientierung ${ }^{73}$ und andererseits durch ein sehr weitgehendes kommunales Engagement aus. Die stadt Nordhorn hat

71 Vgl. ebenda.

72 Vgl. Lange, S.: Telematik - Neue Aufgaben für die kommu- 
im Bereich der Informations- und Kommunikationsanwendungen eine ausgeprägte Promotorrolle übernommen und will diesbezügliche Anwendungen, in Form von weiteren Demonstrationsprojekten $^{74}$, in der Region vorantreiben. ${ }^{75}$

\subsection{Technologie- und Gründerzentren}

Technologie- und Gründerzentren gibt es seit Beginn der 80er Jahre. Im März 1989 gab es 73 solcher Innovationszentren in der Bundesrepublik Deutschland, die am stärksten auf die Länder Baden-Württemberg und Nordrhein-Westfalen konzentriert sind (siehe Abbildung 2.3). 76 Die Technologieund Gründerzentren mit differenziertem Informations- und Kommunikationsangebot stellen mit 25 zentren 77 die dominierende Anwendungsform im Bereich der Informations- und Kommunikations-Anwendungen auf Bundesebene dar. 78 Bei der räumlichen Verteilung dieser Einrichtungen sind die großen

nale wirtschaftspolitik, in: Städte- und Gemeindebund, a.a.0., s. 135 .

73 Bedingt durch die Kooperation mit der Technischen Hochschule in Enschede und durch potentielle Nutzer aus der Industrieregion Twente aufgrund der niedrigeren Gebühren.

74 Beispielsweise im Bereich des Datentransfer zwischen Laborbetrieben und im Bereich der Verkehrslenkung einer internationalen spedition.

75 Vgl. Georgieff, P. U.a.: Lokale und regionale TelematikVorhaben in der Bundesrepublik Deutschland, a.a.0., s. 85 .

76 Vgl. Mock, W.: Stützen des Strukturwandels, Technologiezentren: Ein wichtiger Faktor regionaler Wirtschaftspolitik, in: VDI-Nachrichten Nr. 16, 21. April 1989, S. 22 .

77 Stand September 1986.

78 Vgl. Georgieff, P. U.a.: Lokale und regionale TelematikVorhaben in der Bundesrepublik Deutschland, a.a.o., s. 32 . 
Abbildung 2.3: Technologie- und Gründerzentren ${ }^{79}$

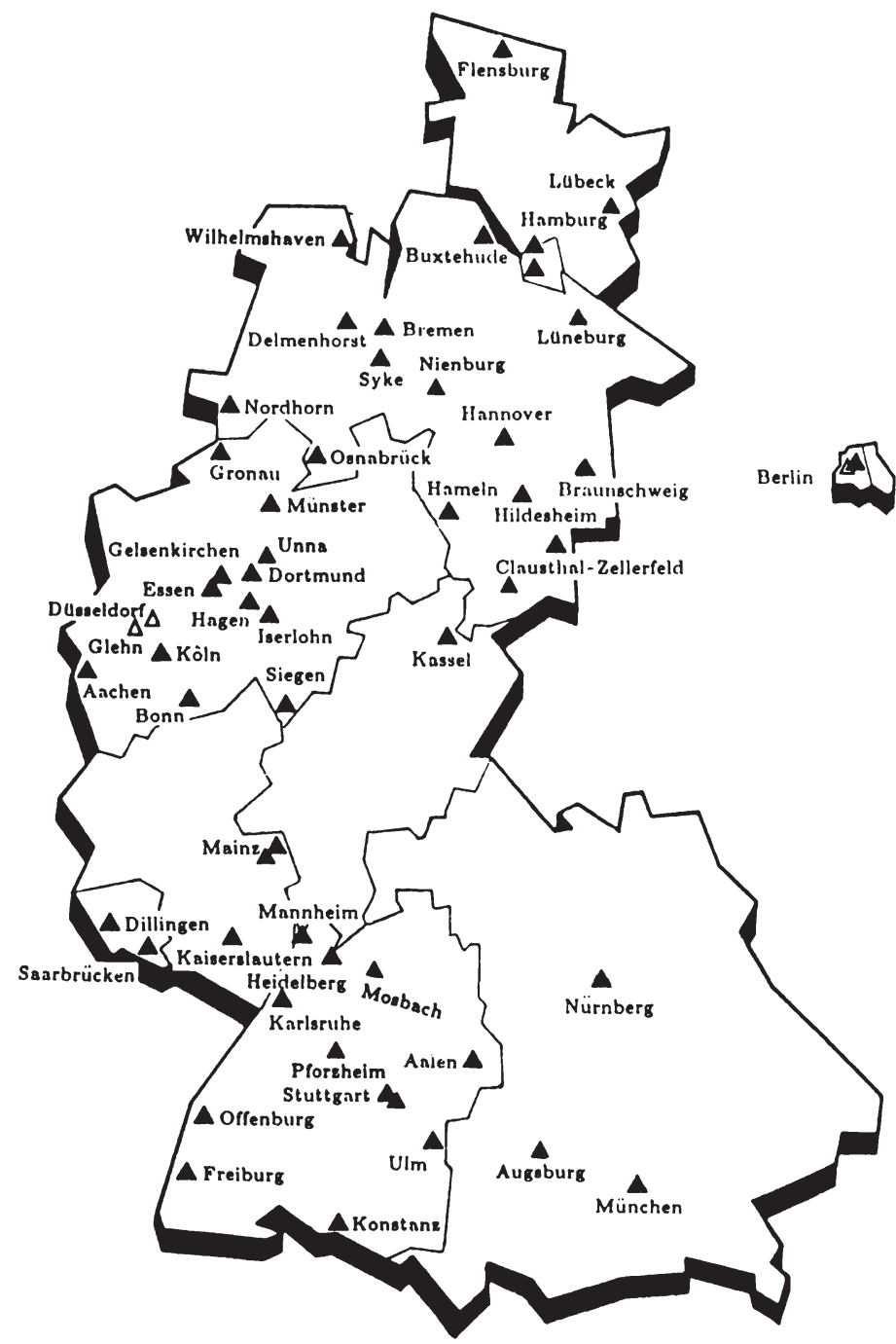

79 Quelle: Mock, W.: Stützen des Strukturwandels, Technologiezentren: Ein wichtiger Faktor regionaler wirtschaftspolitik, a.a.0., s. 22 . 
und mittleren Zentren begünstigt, "da die überörtlichen Fördermittel an das Vorhandensein einer technisch orientierten Universität oder Fachhochschule gebunden sind" 80 .

Technologiezentren, Innovations- und Gründerzentren, Technologiehöfe und -fabriken sind Begriffe, die synonym für die Technolgie- und Gründerzentren verwendet werden. Unter dem Begriff eines Technologie- und Gründerzentrums wird "eine Standortgemeinschaft von relativ jungen und zumeist neu gegründeten stammunternehmen verstanden, deren betriebliche Tätigkeit vorwiegend in der Entwicklung, Produktion und Vermarktung technologisch neuer Produkte, Verfahren und Dienstleistungen liegt" 81 und die im Zentrum auf zentrale

Dienstleistungen wie Telefon-, Post- und Schreibdienste und Informations- und Beratungsangebote 82 zurückgreifen können.

Die ziele der Initiatoren der Technologie- und Gründerzentren sind

- die Intensivierung des Technologietransfers,

- die Schaffung von Synergieeffekten durch eine Zusammenarbeit der im Zentrum ansässigen Unternehmen und

- eine Verbesserung der regionalen wirtschaftsstruktur durch die Ansiedlung von technologie- und wachstumsintensiven Industriezweigen. 83

80 Funck, R.: Technologiepolitik und räumliche struktur, in: Böventer, von E. (Hrsg.): Regionale Beschäftigung und Technologieentwicklung, a.a.0., s. 126.

81 Sternberg, R.: Technologie- und Gründerzentren als Instrument kommunaler Wirtschaftsförderung, a.a.0., s. 86 und Fiedler, H.; Wodtke, K.: Technologiezentren in der Bundesrepublik Deutschland 1988, Berlin 1988, S. $37 \mathrm{f}$.

82 Z.B. im Bereich Marketing, Finanzierung und Buchführung.

$83 \mathrm{Vgl}$. Rürup, B.: Wirtschaftliche und gesellschaftliche Perspektiven der Bundesrepublik Deutschland, a.a.0., s. 86. 
Das Leistungsangebot der Technologie- und Gründerzentren umfaßt in der Regel subventionierte Produktionsräume, gemeinsame Infrastruktureinrichtungen und Informations-84 und Beratungsmöglichkeiten. 85 Die Unternehmen können diese Leistungen meistens maximal fünf Jahre in Anspruch nehmen und müssen sich dann selbständig weiterentwickeln. 86

Ein Beispiel für ein Technologie- und Gründerzentrum mit einem größeren Angebot an neuen Informations- und Kommunikationstechniken stellt das saarbrücker Innovations- und Technologie-zentrum dar. Die Initiative zur Gründung des Zentrums ging von der stadt, insbesondere vom oberbürgermeister aus, wobei vom Land und der Europäischen Gemeinschaft 36 Prozent der voraussichtlichen Gesamtkosten des Projektes getragen werden. Auch die laufenden Kosten der Gemeinschaftseinrichtungen und des Zentrum-Managements werden vom Land und der Europäischen Gemeinschaft mitfinanziert. 87

Dem Zentrum steht, im Verhältnis zu den anderen Zentren in Deutschland, das größte Areal zur Verfügung, das zum Großteil allerdings noch unbebaut ist. Ende 1986 haben im zentrum insgesamt 18 junge Unternehmen gearbeitet, die insgesamt 118 Mitarbeiter beschäftigen. Die Unternehmer stammen überwiegend nicht aus der Region. ${ }^{8}$ zusätzlich sind im zen-

84 Z.B. über mögliche Technologietransfers und Förderungsprogramme.

85 Vgl. Funck, R.: Technologiepolitik und räumliche struktur, in: Böventer, von E. (Hrsg.): Regionale Beschäftiqung und Technologieentwicklung, a.a.0., S. 126.

86 Vgl. Mock, W.: Stützen des Strukturwandels, Technologiezentren: Ein wichtiger Faktor regionaler Wirtschaftspolitik, a.a.o., s. 22 .

87 Vgl. Georgieff, P. U.a.: Lokale und regionale TelematikVorhaben in der Bundesrepublik Deutschland, a.a.o., s. 53 .

$88 \mathrm{Vgl}$. Sternberg, R.: Technologie- und Gründerzentren als Instrument kommunaler Wirtschaftsförderung, a.a.o., S. 167 . 
trum zwei Forschungseinrichtungen der Fachhochschule des Saarlandes angesiedelt.

Die Kaltmiete für Existenzgründer beträgt mit 5,- DM je qm ca. die Hälfte der marktüblichen Kosten und ist zunächst auf drei Jahre befristet. Die Nebenkosten betragen 4,- DM je qm. 89

Als zentrale service-Einrichtungen werden im Zentrum

- eine Telefonzentrale und Anmeldung,

- ein Sekretariat mit Telefax, Telex, Textverarbeitung und Kopiergerät,

- mehrere Personalcomputer mit Drucker,

- eine Bibliothek,

- verschiedene Schulungs- und Besprechungsräume mit audiovisuellen Hilfsmitteln sowie

- eine Kantine angeboten. 90

Das Beratungsangebot des Zentrums umfaßt die Gründerberatung, die Finanzierungsberatung, die Buchhaltung und eine Marketingberatung. Zusätzlich besteht mit dem Institut zur Förderung von Existenzgründungen eine Kooperationsvereinbarung, die eine betriebswirtschaftliche Aus- und Weiterbildungsmöglichkeit für technisch vorgebildete Gründer beinhaltet.91 Eine Technologieberatung wird vom Zentrum direkt nicht angeboten. Entsprechende Beratungsleistungen werden durch die Zentrale für Produktivität und Technologie Saar wahrgenommen.

Das Innovations- und Technologie-Zentrum Saarbrücken siedelt verstärkt Unternehmen im Bereich neuer Informationsund Kommunikationstechniken an. Für die zukunft ist ein Re-

$89 \mathrm{Vgl}$. Georgieff, P. U.a.: Lokale und regionale TelematikVorhaben in der Bundesrepublik Deutschland, a.a.0., s. 55 .

$90 \mathrm{Vgl}$. ebenda S. 54. 
chenzentrum für graphische Anwendungen und Ausbildungslehrgänge im Bereich der Anwendung neuer Informations- und Kommunikationstechniken geplant. Das Zentrum sieht sich als öffentliche Initiative zur Technologieförderung in altindustriellem Gebiet, das unter anderem auch neue Informationsund Kommunikationstechniken fördern will. 92

\subsection{Einschlägige Informations- und Beratungseinrich- tungen}

Einschlägige Informations- und Beratungseinrichtungen in der Bundesrepublik Deutschland im Bereich der Informationsund Kommunikationsanwendungen umfassen räumlich konzentrierte Angebote mit gezielten Informations- und Beratungsleistungen. Neben den Informationstechnik-Zentren der Gesellschaft für Mathematik und Datenverarbeitung existieren einschlägige Informations-, Beratungs- und Transferstellen bei Kammern, Hochschulen und Fachverbänden, die für kleine und mittlere Unternehmen herstellerneutrale Beratung, Information und Demonstration zum Einsatz neuer Informationsund Kommunikationstechniken und -dienste anbieten. 93

Beispielhaft werden im folgenden das InformationstechnikZentrum Köln, das ostfriesische regionale Informationstechnikzentrum und das bayerische Telekommunikationszentrum MüTel dargestellt.

\section{Informationstechnik-zentrum Röln}

Das Informationstechnik-Zentrum Köln ist im gleichen Gebäu-

92 Vgl. ebenda S. 59.

$93 \mathrm{Vgl}$. Gundrum, U.: Regionale Telematikanwendungen in der Bundesrepublik Deutschland, in: Der Städtetag, 11, 1987, S. 677-682. 
de untergebracht wie das Technologiezentrum Köln. Initiiert wurde es von der Kölner Technologierunde und der Gesellschaft für Mathematik und Datenverarbeitung und wird unter der Beteiligung der Stadt Köln, des Erftkreises, des Rheinisch-Bergischen und des oberbergischen Kreises, der Stadt- und Kreissparkasse und der Kölner Bank von 1867 betrieben. 94 Die Anlauf-Verluste werden anteilig vom Land Nordrhein-Westfalen übernommen.

Die Aufgaben des Informationstechnik-Zentrums sind die Information, Schulung und Beratung über die Anwendung der Informationstechnik für Kleinbetriebe, Arbeitnehmer und für die öffentlichkeit im Bereich der Wirtschaftsregion Köln.95 Zusätzlich soll durch Kooperationen mit anderen Ausbildungsinstitutionen und durch gezielte Vermittlungen zwischen Informations- und Kommunikationsanbietern und -Anwendern die regionale Ausbildungs- und Marktsituation verbessert werden. 96

Die Ausstattung des Zentrums umfaßt Teletex, Telefax, Telex, Btx, Datex-P und verschiedene Mikrocomputer mit Software. An Schulungen werden diverse Seminare angeboten, wie

- eintägige Einführungen in die Informationstechnik,

- Grundlagenseminare für alle Adressaten und

- Fachseminare für den Handwerksbereich. 97

94 Vgl. Georgieff, P. U.a.: Lokale und regionale Telematikvorhaben in der Bundesrepublik Deutschland, a.a.o., S. 41.

$95 \mathrm{Vgl.} \mathrm{Minister} \mathrm{für} \mathrm{Wirtschaft,} \mathrm{Mittelstand} \mathrm{und} \mathrm{Technolo-}$ gie des Landes Nordrhein-Westfalen (Hrsg.): Zukunftswerkstatt Nordrhein-Westfalen, Düsseldorf 1989, S. 89.

$96 \mathrm{Vgl.} \mathrm{Georgieff,} \mathrm{P.} \mathrm{U.a.:} \mathrm{Lokale} \mathrm{und} \mathrm{regionale} \mathrm{Telema-}$ tik-Vorhaben in der Bundesrepublik Deutschland, a.a.o., S. 41 .

$97 \mathrm{Vgl}$. ebenda. 
Die Grundlagen- und Fachseminare dauern 2 bis 3 Tage und können durch praktische Übungen und durch betriebsspezifische Beratungen ergänzt werden.

Da das Infomationstechnik-Zentrum seine Tätigkeit erst seit oktober 1986 ausübt, kann über die Zielerreichung des Zentrums noch nicht geurteilt werden. Unabhängig davon ist das einschlägige Dienstleistungsangebot der Region deutlich verbessert worden.

\section{Ostfriesisches regionales Informationstechnikzentrum}

In Anlehnung an das Konzept der Gesellschaft für Mathematik und Datenverarbeitung ist beabsichtigt die Abteilung Informationstechnik der Gemeinnützigen Ausbildungsgesellschaft 98 mit der Übungswerkstatt Datentechnik zum ostfriesischen regionalen Informationstechnikzentrum auszubauen. Mit der Übungswerkstatt als Grundstock soll das Informationstechnik-Zentrum in mehreren Abschnitten fertiggestellt werden.99 Unterstützt wird das Projekt vom Wirtschaftsministerium des Landes Niedersachsen, das im ersten Abschnitt $90 \%$ der Kosten fördert.

Die mit dem Vorhaben verbundenen zielsetzungen sind

- das Auslösen von Impulsen für Modernisierungen der Betriebe,

- die Motivierung zu Erweiterungen des Leistungsangebotes der Betriebe,

- die Förderung von Kooperationen sowie

- die Verbesserung der Anbindung der strukturschwachen Region an das Bundesgebiet, 100

98 Die im Januar 1983 vom Landkreis Aurich gegründet wurde.

99 Vgl. Georgieff, P. U.a.: Lokale und regionale Telematik-Vorhaben in der Bundesrepublik Deutschland, a.a.o., S. 41 .

$100 \mathrm{Vgl}$. ebenda S. 72. 
wobei das Zentrum ingesamt einen Beitrag zur Verminderung der starken Arbeitslosigkeit in Ostfriesland leisten soll. Dies ist durch die Aus- und Fortbildung sowie Umschulung von Arbeitslosen, die individuelle Information und Beratung kleiner und mittlerer Betriebe, die Initiierung und Durchführung von beispielhaften Projekten zur Informations- und Kommunikations-Anwendung in der Region und die Einrichtung eines Telehauses mit umfassendem Technik- und Diensteangebot sowie Telearbeitsplätzen beabsichtigt. Als Adressaten des Leistungsangebots sind im Schulungsbereich überwiegend arbeitslose Jugendliche und im Informations-, Beratungsund Entwicklungsbereich kleine und mittlere Handwerks- und Dienstleistungsbetriebe sowie öffentliche Einrichtungen vorgesehen. Für interessierte Firmen besteht die Möglichkeit, Telearbeitsplätze in Anspruch zu nehmen. 101

Mit dem Ausbau des Leistungsangebots der gemeinnützigen Ausbildungsgesellschaft im Bereich herstellerneutrale Information und Beratung, sollen die Informations- und Kommunikationsanwendungen in den kleinen und mittleren Betrieben der Region verstärkt werden. Eine dauerhafte senkung der Arbeitslosigkeit in der Region wird jedoch mit dem entstehen von technologieorientierten Unternehmen zusammenhängen, die langfristig die im Zentrum ausgebildeten und damit höher qualifizierten Arbeitslosen beschäftigen können.

Erstaunlich ist im Fall des ostfriesischen Informationstechnik-Zentrums das starke Engagement des Landes Niedersachsen. Zusätzlich zur Förderung durch die Gemeinschaftsaufgabe zur Verbesserung der regionalen wirtschaftsstruktur werden Landesmittel im Bereich neuer Informationsund Kommunikationstechniken und -dienste eingesetzt. In strukturschwachen Landesteilen soll gezielt Nachfrage stimuliert werden, um die Deutsche Bundespost zu einem entsprechenden Netz- und Diensteangebot ${ }^{102} \mathrm{zu}$ bewegen. 103

$101 \mathrm{Vgl}$. ebenda S. 74 .

102 Beispielsweise über flexible Netzelemente. 


\section{Bayerisches Telekommunikationszentrum MüTel}

Das bayerische Telekommunikationszentrum MüTel ist im Mai 1989 im Münchner Technologiezentrum angesiedelt worden und gilt als bisher in der Bundesrepublik Deutschland einmalige konzertierte Aktion der Wirtschaft - Hersteller und Nutzer - in Zusammenarbeit mit staatlichen und wissenschaftlichen Institutionen und der Deutschen Bundespost. 104 Neben dem ISDN-Center der Nixdorf AG besteht somit in München die Möglichkeit einer herstellerunabhängigen Telekommunikationsberatung. 105

Das MüTel wurde 1987 unter der Schirmherrschaft des bayerischen Staatsministers für Wirtschaft und Verkehr gegründet. Unterstützt wurde es vor allem vom Förderkreis Neue Technologien, der Industrie- und Handelskammer, der Deutschen Bundespost und dem Freistaat Bayern. 106 Zusätzliche Unterstützung erhält das MüTel, das von der Rechtsform her ein Verein ist, durch seine Mitglieder aus der wirtschaft. Dies sind unter anderen die Firmen Siemens, AT\&T Deutschland, Philips, stollmann und Telenorma. 107

Zielsetzung des MüTels ist eine umfassende und unabhängige Information potentieller Anwender von Telekommunikationsdiensten im ISDN. Vor allem kleine und mittelständische Be-

$103 \mathrm{Vgl.}$ Georgieff, P. U.a.: Lokale und regionale Telematik-Vorhaben in der Bundesrepublik Deutschland, a.a.o., S. 76 .

$104 \mathrm{Vgl}$. Schambeck, H.: Unterstützung für den Mittelstand, in: Frankfurter Allgemeine Zeitung Nr. 241 vom 17.10 .89 , S. B8.

105 Vgl. O.V.: MüTel eröffnet ISDN-Beratungscenter in München, in: ISDN report, Mai 1989.

106 Vgl. O.V.: Informationsblatt MüTel e.V., München 1989.

$107 \mathrm{Vgl}$. Ewe, J.: Otello soll es richten, in: Frankfurter Allgemeine Zeitung Nr. 67 vom 20. März 1990, S. B5. 
triebe und Freiberufler sollen gefördert und in die Lage versetzt werden, geeignete individuelle Telekommunikationsinfrastrukturen aufzubauen. Durch die Einbindung in das Bayerische Technologieberatungsprogramm können die Beratungskosten bis zu 90 Prozent gefördert werden. Schwerpunkt der bayernweiten Tätigkeit ist die schulung und Beratung, die durch eine Dauerausstellung von Telekommunikations-Endgeräten, die zu Modell-Arbeitsplätzen konfiguriert sind, ergänzt wird. 108

Zur Unterstützung der Beratung wurde im MüTel das Beratungsprogramm OTELLO109 entwickelt. Eine Beratung im Rahmen des OTELLO-Programms erfolgt in vier stufen: ${ }^{110}$

\section{OTELLO I - Erstellung eines Telematikkonzeptes}

In der ersten stufe wird eine Analyse der vorhandenen Bürotechnologien, der externen Kommunikationsflüsse und der im Zusammenhang stehenden Kosten durchgeführt. OTELLO I schließt mit einem Grobkonzept ab, das Ansätze zur Effizienzsteigerung und Kostenreduzierung im Verwaltungsbereich darlegt.

\section{OTELLO II - Feinanalyse pro Arbeitsplatz}

otello II beinhaltet die Analyse der internen Kommunikationsbeziehungen und Empfehlungen zur Geräteausstattung. Unter Berücksichtigung der bereits existierenden Endgeräte und des vorgegebenen Investitionsrahmens, werden in einem Feinkonzept die Vorraussetzungen für einen effektiven Telematik-Einsatz dargestellt und

$108 \mathrm{Vgl}$. Schambeck, H.: Unterstützung für den Mittelstand, a.a.0..

109 OTELLO steht für optimierung der Telekommunikation in der betrieblichen Organisation.

$110 \mathrm{Vgl}$. o.V.: ISDN-Kommunikationsmöglichkeiten für die Zukunft - Telekommunikationsberatung für den Mittelstand, in: Deutsche Handwerks Zeitung vom 21. April 1989. 
Empfehlungen für unterschiedliche Arbeitsplatztypen ausgesprochen.

\section{OTELLO III - Hilfestellung bei der Installation}

Mit Hilfe des Feinkonzeptes wird das Gesamtkonzept erstellt, das die Basis für die Einholung von Angeboten darstellt. Nach der Konfigurationsentscheidung durch den Unternehmer wird die Realisierung des Konzeptes von MüTel überwacht und durch Trainings-Seminare im Bereich der ISDN-Geräte-Nutzung unterstützt. OTELLO III soll mit einer funktionsfähigen Kommunikations-Infrastruktur abgeschlossen werden.

\section{OTELLO IV - Betreuung auch nach der Inbetriebnahme}

Die letzte stufe von OTELLO beinhaltet das Controlling. Über einen längeren Zeitraum wird überprüf, ob das ronzept funktionsfähig und noch zeitgemäß ist oder Anpassungen an Neuentwicklungen notwendig werden.

Das Programm OTELLO erlaubt zusätzlich die Durchführung einer ersten 'Blitzanalyse' mit einer technischen und betriebswirtschaftlichen Auswertung. Dies ermöglicht potentiellen Anwendern eine erste Kosten-Nutzen-Abschätzung des ISDN-Einsatzes.

In den Jahren von 1987 bis 1989 konnten durch MüTel mehr als 2000 kleine und mittelständige Unternehmen informiert werden. Vor allem mittelständische Unternehmen, die keine firmeneigenen Kommunikationsfachleute zur verfügung haben, sind auf solche Institutionen angewiesen, die, neben den technischen Möglichkeiten, den betriebswirtschaftlichen Nutzen für das $z u$ beratende Unternehmen herausarbeiten. 111

$111 \mathrm{Vgl}$. Schmidt, E.: Interesse an neuem Fernmeldedienst ist noch gering - ISDN überfordert den Mittelstand, in: VDI-Nachrichten Nr. 21 vom 26. Mai 1989, S. 22. 


\subsubsection{4. Öffentliche Betriebsversuche}

Vor der Einführung von neuen Fernmeldediensten werden durch die Deutsche Bundespost technische Betriebsversuche durchgeführt, die auch als sogenannte Pilotprojekte bezeichnet werden. Die Betriebsversuche der Deutschen Bundespost werden im folgenden unter dem Begriff 'öffentliche Betriebsversuche' zusammengefaßt. 112

Die öffentlichen Betriebsversuche lassen sich grundsätzlich in zwei Formen untergliedern:

- den postbezogenen Test, der aus den verschiedenen firmenindividuellen Lösungen die zukünfige Serientechnik herauskristallisiert und

- den anwenderbezogenen Test, in dem die Anwendungen und ihre Einbindung in den neuen Dienst und die Endeinrichtungen getestet werden und die Versuche aus normungs- und datenschutzmäßiger sicht begleitet werden. 113

Beispiele für öffentliche Betriebsversuche sind der BigfonPilotversuch, der 1984 bundesweit startete und die Kommunikation mit Bildfernsprechverbindungen ermöglicht und die ISDN-Pilotprojekte in Mannheim und stuttgart, in denen 1987, nach der technischen Erprobung einer Mindest-Konfiguration, die Teilnehmer mit verschiedenen Endgeräten am Pilotprojekt teilnahmen. 114

112 Vgl. Georgieff, P. u.a.: Lokale und regionale Telematik-Vorhaben in der Bundesrepublik Deutschland, a.a.0., s. 32 .

113 Vgl. Lydorf, H.: TEMEX - ein neuer Fernmeldedienst der Deutschen Bundespost, in: Bundesminister Schwarz-Schilling, Florian, w. (Hrsg.): Jahrbuch der Deutschen Bundespost 1986, Bad Windsheim 1986, S. 265.

$114 \mathrm{Vgl}$. Lange, S. u.a.: Lokale Initiativen zur Förderung der Anwendungen der Telekommunikation, a.a.0., s. 171. 
Ein neueres Projekt stellt das Berliner Kommunikationssystem BERKOM dar, 115 dessen erster Abschnitt Ende Oktober 1988 offiziell in Betrieb genommen wurde. Das BERKOM-Projekt ist ein gemeinsames Projekt der Deutschen Bundespost und des Berliner senats und sollte ursprünglich Ende 1989 auslaufen. Mittlerweile ist die Laufzeit des Projektes um zwei Jahre verlängert worden. 116

Mit dem Projekt BERKOM soll die Entwicklung von Diensten, Anwendungen und Endsystemen des zukünftigen GlasfaserBreitbandnetzes ISDN-B gefördert werden. Geleitet wird das Projekt von der Deutschen Telepost Consulting GmbH (Detecon).117 Konkrete Anwendungsfelder sind die 'Telemedizin' in der medizinische Einrichtungen für verteilte Diagnoseund Therapie-Aufgaben verknüpft werden, das 'Telepublishing', in dem der körperliche Transport von Druckunterlagen durch Breitband-Kommunikation ersetzt wird und die 'Verteilten Fabriken', in denen getrennte Unternehmensbereiche informationstechnisch vernetzt werden. 118

Bei den öffentlichen Betriebsversuchen der Deutschen Bundespost werden, wie o.a., verschiedene technische Lösungen im Betrieb in unterschiedlichem Umfeld getestet, um daraus eine Auswahl für die Serientechnik zu treffen. Die gezielte Erprobung der Anwendungen überläßt sie den Anwendern. 119 Besonders im Bereich der Schmalband-Kommunikation existie-

115 Vgl. O.V.: Neue Chancen durch Selbstwahl im Glasfasernetz, in: IHK - Mitteilungen, Frankfurt den 15.11.89.

$116 \mathrm{Vgl}$. Der Bundeminister für Forschung und Technologie und der Bundesminister für Wirtschaft (Hrsg.): Zukunftskonzept Informationstechnik, Bonn 1989, S. 219.

$117 \mathrm{Vlg}$. ebenda.

$118 \mathrm{Vgl}$. O.V.: Glasfaser unter der Lupe - Berkom untersucht Breitbandkommunikation, in: VDI-Nachrichten, Berlin den 18.11.1988.

$119 \mathrm{Vgl}$. Lange, S.: Teleports - Entstehung und Förderung der Telematik, a.a.0., S. 289. 
ren nicht in der Technik liegende Engpässe, die eine schnelle Diffusion der neuen Dienste erschweren.120 Die Engpässe konzentrieren sich primär auf die Bereiche:

- Organisationskapazität,

- Akzeptanz beim Personal,

- Angebotsübersicht,

- Teilnehmerübersicht und

- Anwendungssystementwicklung. 121

Der Engpaß Organisationskapazität beinhaltet die Problematik der Unternehmen, Anforderungen an integrierte Systeme der Büro- und Telekommunikation zu formulieren und technisch-organisatorische Gesamtkonzepte zu entwickeln. Nachteile in diesem Bereich dominieren primär bei den mittelständigen Unternehmen, die keine eigenen organisationsabteilungen haben und größere Kosten für die notwendigen Analysen scheuen.

Die langsame Diffusion von neuen Diensten wie Bildschirmtext, Teletex und anderen zeigt, wie lange es dauern kann, bis sich eine neue Technik ihren Markt schafft. 122 Aus diesem Grund sollten verstärkt Anwendungspilotvorhaben, vor allem im Bereich der klein- und mittelständischen Unternehmen, gefördert werden. Unter Anwendungspilotvorhaben sind hierbei weniger öffentliche Betriebsversuche im bisherigen Sinn zu verstehen, sondern vielmehr eine stufe der Weiterentwicklung, Modifikation oder Kombination der Nutzung bereits existierender Diensteangebote zur Befruchtung der Adoptionsbereitschaft ${ }^{123}$ im peripheren Raum. 124 Förderungen in diese Richtung stellen beispielsweise das o.a. bayerische Telekommunikationszentrum MüTel und die Berliner Pro-

$120 \mathrm{Vgl}$. Lange, S. u.a.: Lokale Initiativen zur Förderung der Anwendungen der Telekommunikation, a.a.0., S. 172 .

$121 \mathrm{Vgl}$. ebenda.

$122 \mathrm{Vgl}$. Lange, S.: Teleports - Entstehung und Förderung der Telematik, a.a.0., S. 289. 
jekte dar, die jedoch regional begrenzt und bis auf wenige Ausnahmen auf hochverdichtete, günstige Regionen konzentriert sind.

\subsubsection{Infrastrukturpolitik}

\subsubsection{Gebührenpolitik unter regionalpolitischem Aspekt}

Für die Preise von Telekommunikationsdiensten ist der ordnungspolitische Rahmen des Fermeldewesens von entscheidender Bedeutung. Die Deutsche Bundespost muß bei der Festlegung der Telekommunikationsgebühren verschiedene ökonomische und außerökonomische zielsetzungen berücksichtigen, wobei die regionalpolitischen ziele zur Begründung der Tarifeinheit im Raum, des Nahbereichskonzeptes und weiterer Merkmale der Tarifstruktur angeführt werden. 125 Beispielsweise enthält der Beschluß des Bundesrates zur Informationstechnik vom 16. November 1984 die Aufforderung an die Deutsche Bundespost, den Bundesländern die Ausbauplanung frühzeitig mitzuteilen und mit ihnen abzustimmen, sowie re-

123 Mit dem Begriff 'Adoption' kann der Vorgang des Vertragsschlusses bezeichnet werden, während die Teilnahme zahlreicher Individuen oder Unternehmen an einem Telekommunikationsdienst als eine Anpassungserscheinung (=Adaption) an eine technische Entwicklung betrachtet wird. In terminologischer Übereinstimmung mit der Akademie für Raumforschung und Landesplanung wird unter 'Adoption' die tatsächliche Teilnahme an einem Dienst verstanden.

124 Vgl. Klaus-Stöhner, U.; Zabel, J.: Möglichkeiten und Anwendungsbarrieren für den Einsatz neuer Informationsund Kommunikationstechniken in einer peripheren Region: Fallstudie Waldeck-Frankenberg, in: Akademie für Raumforschung und Landesplanung (Hrsg.): Räumliche Wirkungen der Telematik, a.a.0., S. 410 .

$125 \mathrm{Vgl}$. Schnöring, T.: Wirkungen der Telekommunikationsentwicklung auf Anwender, a.a.0., S. 85. 
gionalpolitische Gesichtspunkte bei der Aufstellung der Planungen $\mathrm{zu}$ berücksichtigen. 126

Die regionalpolitischen Forderungen gegenüber der Deutschen Bundespost enthalten folgende Punkte:

- die flächendeckende Bereitstellung der technischen Telekommunikationsinfrastruktur mit Einführung der Dienste,

- das flächendeckendes Angebot aller Telekommunikationsdienste bei gleicher qualität,

- die Berücksichtigung regionalpolitischer ziele bei der Einführung neuer Dienste und

- die Berücksichtigung regionalpolitischer ziele bei der Gebührenfestlegung. 127

\section{Prinzip der Gebühreneinheit im Raum}

Nach dem Grundgesetz und dem Fermeldeanlagengesetz hat die Deutsche Bundespost das Recht, Fernmeldeanlagen zu betreiben und $\mathrm{zu}$ errichten. Nach dem Postverwaltungsgesetz obliegt ihr somit die pflicht, ihre Anlagen der modernen Technologieentwicklung anzupassen und sich bei ihrem Handeln nach den verkehrs-, wirtschafts-, finanz- und sozialpolitischen Vorgaben der Bundesregierung zu richten. 128

Ausgehend von der Infrastrukturaufgabe der Deutschen Bundespost, läßt sich das Prinzip der Gebühreneinheit im Raum ableiten. Es beinhaltet, daß der gleiche Dienst an verschiedenen orten zum gleichen Preis angeboten werden muß. Bedingt durch die unterschiedlichen topographischen Bedingungen, die unterschiedliche Bevölkerungsdichte und die

126 Vgl. Schnöring, T.; Thomas, K.: Regionalpolitische Aspekte beim Angebot von Telekommunikationsdiensten, in: Bundesminister Schwarz-Schilling, Florian, W. (Hrsg.): Jahrbuch der Deutschen Bundespost 1985, Bad Windsheim 1985 , S. 552 .

$127 \mathrm{Vgl}$. ebenda.

$128 \mathrm{Vgl}$. ebenda S. 553. 
unterschiedliche Intensität der Nutzung, stellt das Prinzip der Gebühreneinheit im Raum eine interregionale Subventionierung dar. "Allein von der Kostenseite aus gesehen bevorteilt das Prinzip der einheitlichen Gebühren im Raum (einheitliche Anschlußgebühr, Grundgebühr, Orts- und Ferngesprächsgebühr) die ländlich peripheren Regionen und benachteiligt die Ballungsgebiete."129

\section{Nahbereichskonzept}

Aufgrund der großen Disparitäten zwischen der siedlungsdichte in den einzelnen orstnetzen - früher konnte innerhalb eines ortsnetzes entfernungs- und zeitunabhängig für eine Gebühreneinheit telefoniert werden - konnten beispielsweise im Ortsnetz Hamburg 25.362 mal mehr Fernsprechanschlüsse zur billigsten Gebühr erreicht werden, als im kleinsten ortsnetz Torfhaus. 130 Dies führte in den $70 \mathrm{er}$ Jahren zur Entwicklung des Konzeptes der Nahbereiche, welches drei wesentliche Hauptziele hatte:

- den Abbau der Disparitäten der zum ortstarif erreichbaren Teilnehmer,

- den Abbau der Tarifierungsprobleme zwischen den Vororten und den Großstädten, die verstärkt zu Ferngesprächsgebühren geführt werden mußten und

- das sämtliche Telefonteilnehmer ihre zuständige Gemeindeverwaltung zum ortstarif erreichen können. 131

Nach der Einführung des Nahbereichs 1980 reduzierte sich die Diskrepanz der erreichbaren Teilnehmer zum ortsgesprächstarif zwar erheblich, enthielt jedoch immer noch starke streuungen. Dies führte $1984 \mathrm{zu}$ einer weiteren Veränderung des Nahbereichskonzeptes. Das Zonenrandgebiet und

129 ebenda S. 554 .

$130 \mathrm{Vgl}$. Schnöring, T.: Wirkungen der Telekommunikationsentwicklung auf Anwender, a.a.0., S. 85.

$131 \mathrm{Vgl}$. ebenda S. 85-86. 
strukturschwache ortsnetze 132 erhalten seit dem 01 . Juli 1985 bis zu fünfzig kostenfreie Gesprächsgebühreneinheiten je Monat, als Ausgleich für die strukturbedingten Nachteile. 133

Die momentanen Gebühren der Telekommunikationsdienste der Deutschen Bundespost stellen eine interregionale Subventionionierung dar, da die Gebühren unter Kostenaspekten im Nahbereich zu niedrig und im Bereich der Ferngespräche zu hoch sind. Unter der gegenwärtigen Tarifstruktur bevorzugt dagegen das Prinzip der Tarifeinheit im Raum die ländlichen Regionen und benachteiligt die Ballungsgebiete. 134

\subsubsection{Entwicklungstendenzen und raumstrukturelle Rele- vanz der Telekommunikationsgebühren}

Die Deutsche Bundespost steht mit ihrer Gebührenpolitik unter einem wachsenden Wettbewerbsdruck, der durch die ausländischen Fernmeldeverwaltungen/-unternehmen bedingt ist. In den USA und anderen Ländern kam es zu der Entstehung konkurrierender Fernmeldeunternehmen, die einen Wettbewerbsmarkt für internationale Telekommunikationsdienste entstehen ließen. Als Folge dieses Wettbewerbs sind die Gebühren besonders auf der verkehrsintensiven Transatlantikroute zurückgegangen. Durch die Veränderungen im Ausland steht die Deutsche Bundespost unter einem immer stärker werdenden internationalen Performance-Vergleich, was einem

132 Strukturschwache ortsnetze sind ortsnetze mit weniger als 30.000 im Nahbereich erreichbaren Telefonhauptanschlüssen.

133 Vgl. Schnöring, T.: Wirkungen der Telekommunikationsentwicklung auf Anwender, a.a.0., S.87.

134 Vgl. Schnöring, T.: Telekommunikationsgebühren und Raumordnung, Diskussionspapiere des Wissenschaftlichen Instituts für Kommunikationsdienste der Deutschen Bundespost, $\mathrm{Nr}$. 23, Bad Honnef 1987, S. 13. 
indirekten Wettbewerb mit ausländischen Fermeldeunternehmen, bezüglich des Niveaus und der struktur der Gebühren, impliziert. 135

Bei den gegenwärtigen Diskussionen über Tarifänderungen sind besonders drei punkte relevant: 136

1. Die Fernsprechgebühren sind durch die interne Subventionierung der Deutschen Bundespost im Verhältnis zu den Kosten zu hoch.

2. Im Bereich der Fernsprechtarife existiert eine starke Kostenüberdeckung bei Ferngesprächen im Inland und demgegenüber eine Kostenunterdeckung bei den Nahbereichsgesprächen.

3. Die technologische Entwicklung im Fernmeldewesen führt zukünftig dazu, daß die 'Dichte' von Verkehrsbeziehungen zunehmend zum dominierenden Kostenfaktor gegenüber der Entfernungskomponente wird.

Mit zunehmender Kenntnis der Kunden über diese Effekte, wächst der Änderungsdruck auf die Gebührenpolitik. Wobei die Kunden mit überhöhten Gebühren versuchen, aus dem Umverteilungssystem auszusteigen. Dies geschieht in Form von privaten Netzen auf Mietleitungsbasis.

Die Deutsche Bundespost hat bereits 1985 erklärt, die Fernsprechtarife längerfristig stärker kostenorientiert weiterzuentwickeln, wobei eine völlige Entfernungsunabhängigkeit in naher zukunft nicht realisierbar ist, da damit eine exorbitante Steigerung der Anzahl an Ferngesprächen zu erwarten wäre. Bei völliger Entfernungsunabhänigigkeit der Tarife, müßte nach Berechnungen der Deutschen Bundespost ein

$135 \mathrm{Vgl}$. Schnöring, T.: Telekommunikationsgebühren und Raumordnung, in: Akademie für Raumforschung und Landesplanung (Hrsg.): Räumliche Wirkungen der Telematik, a.a.o., s. 150 .

$136 \mathrm{Vgl}$. ebenda. 
Einheitszeittakt von ca. einer Minute (für alle Gespräche) eingeführt werden, um einnahmenneutral zu sein. 137

Die Problematik einer kostenorientierten Tarifausrichtung, aus der sicht der Raumordnung, besteht darin, daß das Prinzip der Tarifeinheit im Raum aufgehoben werden müßte und ein nach Routen beziehungsweise Verkehrsbeziehungen differenziertes Tarifsystem eingeführt werden muß. Dies würde die Kommunikation mit und vor allem zwischen den Agglomerationen relativ zur Kommunikation zwischen ländlichen Regionen begünstigen. Insgesamt gesehen scheint sich jedoch der Einfluß der regionalpolitischen ziele auf die Tarifstruktur zu reduzieren, da dem Wettbewerb im Telekommunikationssektor eine zunehmende Bedeutung beigemessen wird. 138

\section{Raumstrukturelle Relevanz der Telekommunikationsgebuhren}

Zur Analyse der Relevanz der Telekommunikationsgebühren für Betriebe in peripheren Regionen und des Einflusses auf die Standort- und Wettbewerbsbedingungen der peripheren Regionen, sind Informationen über die Belastung von Unternehmen, Betrieben und Verwaltungen mit Telekommunikationsgebühren notwendig. Eine, von der Bundesforschungsanstalt für Landeskunde und Raumordnung durchgeführte, Literaturrecherche zu diesem Thema kommt zu dem Resultat, daß die Kenntnisse diesbezüglich außerordentlich dürftig sind und sich Aussagen auf diesem Gebiet zum großen Teil im Spekulativen bewegen müssen. 139

$137 \mathrm{Vgl}$. Deutscher Bundestag: Bundestagsdrucksache 10/5913, Bonn 1986.

$138 \mathrm{Vgl}$. Schnöring, T.: Telekommunikationsgebühren und Raumordnung, in: Akademie für Raumforschung und Landesplanung (Hrsg.): Räumliche Wirkungen der Telematik, a.a.0., S. 152 .

$139 \mathrm{Vgl}$. Schnöring, T.: Wirkungen der Telekommunikationsentwicklung auf Anwender, a.a.0., s. 95. 
Grundsätzlich kann jedoch die begründete Vermutung ausgesprochen werden, daß Betriebe in peripheren Regionen "anteilig mehr Telekommunikationsdienste über größere Entfernungen nachfragen und deshalb infolge der Distanzabhängigkeit der Gebühren mehr für ihre Kommunikation aufwenden müssen"140. Dies ermöglicht allerdings keine Aussagen bezüglich der Relevanz dieser Mehrkosten für die Wettbewerbsfähigkeit.

Eine Untersuchung über die reinen Fernmeldegebühren liegt für die Jahre 1978 bis 1980 für ausgewählte Wirschaftssektoren vor. Nicht enthalten sind in der Untersuchung die sonstigen Kommunikationskosten für die Telekommunikationsendgeräte und die bei der Kommunikation anfallenden Personalkosten. Tabelle 2.1 zeigt, daß die Fernmeldegebühren bei mehr als funfzig prozent der sektoren weit unter einem Prozent liegen und lediglich bei den Handelsvermittlungen und den Reisebüros über zwei prozent liegen.

Die Ergebnisse des Vergleichs der Fernmeldegebührenanteile an den Gesamtkosten der Unternehmen deuten darauf hin, daß die Mehrbelastung der peripheren standorte mit Fernmeldegebühren, im Vergleich $z u$ den anderen dominierenden Kostenfaktoren, lediglich einen geringen Einfluß auf die Wettbewerbsfähigkeit hat.

140 Schnöring, T.: Telekommunikationsgebühren und Raumordnung, in: Akademie für Raumforschung und Landesplanung (Hrsg.): Räumliche Wirkungen der Telematik, a.a.o., S. 153 . 
Tabelle 2.1: Vergleich der Fernmeldegebührenanteile ausgewählter Wirtschaftsbereiche in $\%$ der Gesamtkosten im Durchschnitt der Jahre 1978-1980141

\begin{tabular}{|l|c|}
\hline Ausgewählte Wirtschaftssektoren & Gesamtkosten in $\%$ \\
\hline Einzelhandel & $0,2 \%$ \\
\hline Großhandel & $0,2 \%$ \\
\hline Gemeinden & $0,2 \%$ \\
\hline Industrie & $0,3 \%$ \\
\hline Banken & $0,3 \%$ \\
\hline Verlage & $0,6 \%$ \\
\hline Privatversicherungen & $1,0 \%$ \\
\hline Freie Berufe & $1,3 \%$ \\
\hline Informations- und Beratungsdienste & $1,5 \%$ \\
\hline Handelsvermittlung & $2,2 \%$ \\
\hline Reisebüros & $3,0 \%$ \\
\hline
\end{tabular}

Die Kommunikationskosten sind somit als standortfaktor für die überwiegende Zahl von Unternehmen nicht von sehr großer Bedeutung. Andererseits können höhere Telekommunikationskosten im ländlichen Raum ein Anwendungshindernis für die Diffusion neuer Dienste darstellen. Dies gilt im besonderen für die neuen Telekommunikationsdienste, die als Netzinfrastruktur das Fernsprechnetz nutzen.

Außerordentlich gravierend ist der Gebührensprung im Fernsprechnetz zwischen der Nahtarifzone und der Fernzone I, der um den Faktor acht steigt. Tabelle 2.2 stellt die Entfernungsabhängigkeit der Gebühren für die Telekommunikationsdienste Btx, Datex-P, Telex, Teletex, Datex-I, Telefax

141 In Anlehnung an Weitzel, G. u.a.: Post- und Fernmeldegebühren in ausgewählten Wirtschaftsbereichen, München 1983, S. 269. 
und Datenübertragung im Fernsprechnetz dar. Grundlage der Gebührenerhebung sind die unterschiedlichen Entfernungszonen. Bei den Diensten Telefax und Datenübertragung im Fernsprechnetz ist, identisch zum Telefonnetz, von vier Entfernungszonen auszugehen. Beim Teletex und Datex-L gibt es drei Entfernungszonen, beim Telex zwei und beim Btx und Datex-P keine. 142

Tabelle 2.2: Entfernungsabhängigkeit von Telekommunikationsdiensten 143

\begin{tabular}{|c|c|c|}
\hline Telekommunikationsdienst & Entfernungszonen & $\begin{array}{l}\text { Gebührengefälle } \\
\text { im Normaltarif }\end{array}$ \\
\hline Btx, Datex-P & 1 entfernungsunabhängig & - \\
\hline Telex & $\begin{array}{c}2 \text { - innerhalb des } \\
\text { zVst-Bereich } 144 \\
\text { - außerhalb des } \\
\text { zvst-Bereichs }\end{array}$ & $\begin{array}{l}1 \\
1,5\end{array}$ \\
\hline Teletex, Datex-L & $\begin{aligned} 3 & \text { - unter } 50 \mathrm{~km} \\
& -50-100 \mathrm{~km} \\
& - \text { über } 100 \mathrm{~km}\end{aligned}$ & $\begin{array}{l}1 \\
1,4 \\
1,7\end{array}$ \\
\hline $\begin{array}{l}\text { Telefax, Datenübertra- } \\
\text { gung im Fernsprechnetz }\end{array}$ & $\begin{array}{l}4 \text { - Nahbereich } \\
\text { - } 20-50 \mathrm{~km} \\
-50-100 \mathrm{~km} \\
\text { - über } 100 \mathrm{~km}\end{array}$ & $\begin{array}{r}1 \\
8 \\
24 \\
32\end{array}$ \\
\hline
\end{tabular}

Relativiert wird die Entfernungskomponente der Telekommunikationsdienste im ISDN durch die erheblich höhere Übertragungsgeschwindigkeit, die es ermöglicht, bestimmte Übertra-

142 Vgl. Kordey, N.: Gebührenpolitik im Fernmeldewesen und Raumordnung, in: Bundesforschungsanstalt für Landeskunde und Raumordnung (Hrsg.): Raumforschung und Raumordnung, Heft 5/6, Bonn, Bad Godesberg 1988, S. 216.

143 In Anlehnung an Kordey, N.: Gebührenpolitik im Fernmeldewesen und Raumordnung, a.a.0., S. 217.

144 Zentralstellenvermittlungsbereiche gibt es insgesamt acht in der Bundesrepublik Deutschland: In Hamburg, Berlin, Hannover, Düsseldorf, Frankfurt, stuttgart, Nürnberg und München. 
gungen in so kurzer zeit abzuwickeln, daß die stark entfernungsabhängigen Tarife nur bedingt zur Wirkung kommen. 145

Unter diesen Aspekten wird aus raumordnungspolitischer sicht lediglich die stark entfernungsabhängige Gebührenregelung im Fernsprechnetz negativ beurteilt. Die im Zuge der technischen Entwicklung reduzierte Entfernungsabhängigkeit der Übertragungskosten muß von der Deutschen Bundespost bei der Festlegung der Entfernungszonen verstärkt berücksichtigt werden. Von besonderer Relevanz ist eine Gebührensenkung im mittleren Entfernungsbereich, der etwa dem Einzugsbereich entspricht. Ein weiterer, äußerst problematischer Faktor sind die entfernungsabhängigen Einwählgebühren bei Datex-P und Telebox, die das Telefon als zubringer nutzen. Neben den entfernungsunabhängigen Übertragungskosten zwischen Netzknoten und Datenbank, fallen für die Überbrückung der Entfernung zwischen Unternehmen und Netzknoten entfernungsabhängige Gebühren an. Dies betrifft im besonderen die peripheren Regionen, da aus ökonomischen Gründen die Netzknoten in den Nachfragezentren angesiedelt sind. 146

Unter diesen Aspekten scheint letztendlich eine flächendeckende Bereitstellung aller Telekommunikationsdienste und ihre entsprechende Anwendung für die peripheren standorte von erheblich größerer Bedeutung $z u$ sein, als die regionalen Differenzen in der Belastung der Wirtschaft mit Telekommunikationsgebühren. 147

$145 \mathrm{Vgl}$. Langen, B., Siemen, A.: ISDN und Spezialnetze. Wo liegen die voraussichtlichen Nutzungsschwerpunkte des ISDN innerhalb eines Datenübermittlungssystems?, in: Office Management, Heft 6, 1988, S. 14-20.

$146 \mathrm{Vgl}$. Kordey, N.: Gebührenpolitik im Fernmeldewesen und Raumordnung, a.a.0., s. 224 .

147 Vgl. Schnöring, T.: Telekommunikationsgebühren und Raumordnung, in: Akademie für Raumforschung und Landesplanung (Hrsg.): Räumliche Wirkungen der Telematik, a.a.o., s. 153 . 


\subsubsection{Regionale Infrastrukturpolitik}

Grundgesetz und Fernmeldeanlagengesetz weisen der Deutschen Bundespost das Recht $z u$, Fermeldeanlagen $z u$ errichten und zu betreiben. Nach dem Postverwaltungsgesetz erwächst daraus die Verpflichtung, eine gleichmäßige Versorgung des ganzen Landes mit modernen Kommunikationsdiensten zu ermöglichen. Ergänzt wird dieser Auftrag durch die Verpflichtung der Deutschen Bundespost zur Eigenwirtschaftlichkeit. Daraus resultiert der bedarfsorientierte Ansatz der Deutschen Bundespost beim Ausbau der ISDN-Netzinfrastruktur. 148 Unter dem Aspekt der schnellen Amortisation der Investitionen, soll der Ausbau in den Ballungszentren beginnen, da der Investitionsaufwand in dünn besiedelten Gebieten höher ist als in den Agglomerationen und zusätzlich in den Ballungsgebieten mit einem höherem Kommunikationsbedarf gerechnet wird. 149

Geht man von der Überlegung aus, daß die Regional- bzw. Raumordnungspolitik keine grundlegende Änderung der räumlichen Prioritäten beim ISDN-Netzinfrastruktur-Ausbau bewirken kann, verbleiben der regionalen Infrastrukturpolitik als Einflußbereiche

- die Sicherstellung der Bereitstellung von provisorischen Anschlußmöglichkeiten für peripher gelegene Nutzer ${ }^{150}$ und

- die Maßnahmen zur Einführung einer Tarifstruktur, die mit den regional- bzw. raumordnungspolitischen zielen ver-

$148 \mathrm{Vgl}$. Schnöring, T.; Thomas, K.: Regionalpolitische Aspekte beim Angebot von Telekommunikationsdiensten, in: Bundesminister Schwarz-Schilling, Florian, W. (Hrsg.): Jahrbuch der Deutschen Bundespost 1985, a.a.0., S. 552.

$149 \mathrm{Vgl}$. Reese, J.: Die Bedeutung der neuen Fernmeldedienste für Nordhessen, Gutachten im Auftrag der Hessischen Staatskanzlei, a.a.0., s. 42 .

150 Beispielsweise mittels sogenannter 'Multiplexer' oder 'Konzentratoren'. 
einbar sind. 151

Für Hessen beispielsweise ist die ISDN-Einführung im Knotenvermittlungsbereich für die Zeiträume von 1991 bis 1993 vorgesehen. Voraussetzung dafür ist jedoch, daß die Deutsche Bundespost diese Termine einhalten kann, was angesichts der Terminverschiebungen der letzten Jahre nicht als gesichert erscheint. Regionalpolitisch relevant wird dann besonders die Prioritätenliste der hessischen städte, die die Reihenfolge der ISDN-Einführung bestimmt. Berücksichtig man zusätzlich, daß sich allein im Fernmeldeamtsbezirk Kassel sechs dislozierte Knotenvermittlungsstellen mit insgesamt 46 ortsvermittlungsstellen befinden, wird deutlich, daß die überwiegende Zahl dieser ortsvermittlungsstellen im nächsten Jahrzehnt nicht digitalisiert werden kann.152

Die Deutsche Bundespost hat aus diesen Gründen die Möglichkeit der ISDN-Fernschaltungen vorgesehen. 153 Diese, auch als Fremdanschaltung bezeichneten, Maßnahmen sollen bis 1990 innerhalb der betreffenden ortsnetzbereiche und $a b$ 1991 auch über diesen Bereich hinaus dafür sorgen, daß die ISDN-Nachfrage gedeckt werden kann. 154 Für Fremdanschaltungen sind jedoch erhebliche Aufwendungen nötig. Allgemein gilt, daß je höher der erforderliche Mindestbedarf an Fernschaltungen ist, desto größer wird die Gefahr, daß potentielle Teilnehmer in noch nicht regulär angeschlossenen Ge-

151 Vgl. Fritsch, M.; Ewers, H.: Telematik und Raumentwicklung, Bonn 1985, S. 81 .

$152 \mathrm{Vgl}$. Reese, J.: Die Bedeutung der neuen Fernmeldedienste für Nordhessen, a.a.0., S. 42 f.

$153 \mathrm{Vgl}$. Bundesminister für das Post- und Fernmeldewesen: ISDN- die Antwort der Deutschen Bundespost auf die Anforderungen der Telekommunikation von morgen, Bonn 1984 , S. 19 .

$154 \mathrm{Vgl}$. Bundesministerium für das Post- und Fernmeldewesen (Hrsg.): Das ISDN-Angebot der Deutschen Bundespost, a.a.0., s. 11 . 
bieten über längere zeit von der Nutzung ausgeschlossen bleiben. 155

Aus regionalpolitischer sicht ist vor allem ein möglichst schneller Ausbau der Telekommunikations-Vermittlungsnetze, insbesondere des Schmalband-ISDN, notwendig. Dadurch würde sich, neben den erwarteten gesamtwirtschaftlichen Produktivitätssteigerungen, der zeitliche Vorsprung der zuerst angeschlossenen Regionen verringern.

\subsection{Beurteilung der regionalen wirtschaftspolitik unter dem Aspekt veränderter Rahmenbedingungen}

\subsubsection{Veränderung der Rahmenbedingungen}

\subsubsection{Allgemeine Aspekte}

Die regionale Wirtschaftspolitik der Bundesrepublik Deutschland, die weitgehend durch die Gemeinschaftsaufgabe 'Verbesserung der regionalen Wirtschaftsstruktur' geprägt wird, ist in den letzten Jahren zunehmend hinterfragt worden. Ausgangspunkt sind die Veränderungen der gesamtwirtschaftlichen Wachstums- und Beschäftigungsbedingungen. Der Rückgang des 'mobilen Potentials'156 und das Entstehen neuer Problemgebiete in Form der altindustrialisierten Regionen begrenzen zunehmend die wirksamkeit der Förderun-

155 Vgl. Fritsch, M. Ewers, H.: Telematik und Raumentwicklung, a.a.0., S. 82 .

156 Die Verringerung der gesamtwirtschaftlichen Wachstumsraten führen zu einer Reduzierung der räumlichen Ausbreitungseffekte. Schwach entwickelte Gebiete können somit kaum noch auf spill-overs aus Ballungsgebieten hoffen. 
gen. 157 Weitere bedeutende Veränderungen sind die Verknappung öffentlicher Mittel, das abnehmende Vertrauen in die Wirksamkeit rein staatlicher Regionalpolitik 'von oben' und die verstärkte Forderung nach regionaler Partizipation an der alltäglichen Planung. 158

Die räumliche Entwicklung in der Bundesrepublik Deutschland ist differenzierter geworden. Ländliche Räume sind grundsätzlich nicht mehr identisch mit strukturschwachen Räumen, und Agglomerationen sind nicht zwangsläufig strukturstark. Dies gilt im besonderen unter dem Aspekt der Arbeitslosenquote159, die sich in bestimmten 'altindustrialisierten' Regionen (z.B. Bremen, Ruhrgebiet und Teilen des Saarlandes) denen der 'klassischen' strukturschwachen Gebiete (z.B. Emsland, Ostfriesland, Eifel und Bayerischer Wald) angenähert hat. 160 Parallel zu dieser Entwicklung verläuft die Wohlstandsentwicklung, die in den altindustrialisierten Regionen seit Mitte der 70er Jahre zurückbleibt. 161

157 Vgl. Buchner, W.: Neuorientierung der regionalen Strukturpolitik, in: Raumforschung und Raumordnung, Heft 4, 1985 , S. 169.

158 Vgl. Stöhr, W.: Ansätze zu einer neuen Fundierung der Regionalpolitik, in: Jahrbuch der Regionalwissenschaft, 1984 , S. $8 \mathrm{ff}$.

159 Unterschiedliche Arbeitslosenquoten sind selbstverständlich nur ein Kriterium zur Bestimmung der regionalen Wirtschaftskraft und von Wohlstandsunterschieden.

$160 \mathrm{Vgl}$. Fleckenstein, K.: Aufgabenteilung in der regionalen Wirtschaftsförderung, in: Raumforschung und Raumordnung, Heft 4, 1985, S. 173; Deutscher Bundestag: Raumordnungsbericht 1986, a.a.o., S. 13 .

$161 \mathrm{Vgl}$. Deutscher Bundestag: Raumordnungsbericht 1986, a.a.O., S. 49 . 


\subsubsection{Veränderung des betrieblichen standortverhaltens}

Seit 1969 ist die Zahl der großräumigen industriellen Neuansiedlungen, Verlagerungen und Zweiggründungen in der Bundesrepublik Deutschland, in der summe aller drei Bereiche, zurückgegangen. 162 Nach dem gravierenden Rückgang in den Jahren von 1969 bis 1974 in allen drei Industrieansiedlungsbereichen ist bis 1983 und seither fast unverändert ein geringes Ansteigen im Bereich Neugründungen, eine leichte Reduzierung im Bereich Zweiggründungen und eine starke Reduzierung im Bereich Verlagerungen festzustellen. 163

Die Verlagerung bzw. Neuansiedlung von Großbetrieben ist inzwischen ein Ausnahmefall geworden und somit ihre großräumige strukturbestimmende und -verändernde Wirkung weitgehend verschwunden. 164 "Der Standortvorteil der Bundesrepublik dürfte sich künftig eher noch stärker auf Faktoren gründen, die bisher noch eng mit Agglomerationsvorteilen verbunden sind. 165 Dies dürfte die Chancen der regionalen strukturpolitik erschweren, zukunftsträchtige Investitionen in wirtschaftsschwache Regionen zu bringen."166

$162 \mathrm{Vgl}$. Bade, F.: Industrieansiedlung in der Bundesrepublik Deutschland - Umfang und regionalpolitische Bedeutung, in: Wochenbericht des Deutschen Instituts für Wirtschaftsforschung, Heft 8, 1984, S. 92.

$163 \mathrm{Vgl}$. ebenda.

164 Die letzte amtliche Zählung der Bundesanstalt für Arbeit weist für 1981 lediglich noch 23 überregionale Verlagerungen von Industriebetrieben mit mehr als 16 Beschäftigten nach. (Vgl. Bade, F.: Industrieansiedlung in der Bundesrepublik Deutschland, a.a.0., s. $92 \mathrm{ff.}$ )

165 Wie Humankapital, Technologie, organisatorisches Wissen, Informations- und Kommunikationssysteme etc..

166 Deutscher Bundestag: Siebzehnter Rahmenplan der Gemeinschaftsaufgabe 'Verbesserung der regionalen Wirtschaftsstruktur', a.a.0., s. 8 . 
Kleinräumliche verlagerungen finden unverändert im Bereich stadt-Umland-Wanderung statt und sind auf die Verdichtungsräume konzentriert. Dabei verlagern über zwei Drittel der Industriebetriebe ihren standort lediglich über äußerst kleine Distanzen rund um den alten standort. 167 Hierbei werden zunehmend höhere standortanforderungen gestellt, als dies in den vergangenen Jahren der Fall war. Die Nähe zur s-Bahn-, Straßenbahn und Autobahn, die hohe Verfügbarkeit von qualifizierten Arbeitskräften und die Gewerbesteuerhöhe sind zu dominierenden Kriterien bei der wahl neuer standorte im Umland geworden. 168

Die Entwicklungsrichtung der regionalen wirtschaftsstruktur läßt sich nicht endgültig prognostizieren. Es liegt jedoch nahe, daß sich die bisherige Entwicklung nicht wesentlich ändern wird. Weiterhin werden vor allem die Ballungsränder von den Veränderungen in den Zentren profitieren, in denen zumindest im Fertigungsbereich ein Verlust an Arbeitsplätzen zu erwarten ist. Da der Ballungsrand noch so viele Agglomerationsvorteile bietet, daß eine Ansiedlung auch dispositiver Aktivitäten als lohnenswert erscheint, werden die Ballungsränder langfristig qualitativ und quantitativ aufgewertet. 169

$167 \mathrm{Vgl}$. Bullinger, D.: Tendenzen betrieblichen Standortwechsels in Ballungsräumen, in: Raumforschung und Raumordnung, Heft 3,1983, S. 82 .

$168 \mathrm{Vgl.}$ o.V.: Steuern + standort, in: Wirtschaftswoche, Nr. 42 vom 14.10.1988, S. 66.

169 Vgl. Bade, F.: Räumliche Anpassungsprozesse, Arbeitsteilung und unternehmerisches standortverhalten, a.a.o., S. 139 f. 


\subsubsection{Konsequenzen für die regionale wirtschaftspolitik}

\subsubsection{Bedeutungsgewinn der Bauleit- und Regionalplanung}

Unter dem Aspekt des zurückgegangenen Neuansiedlungs- und Verlagerungspotentials im Industriebereich existieren für peripher gelegene, wirtschaftsschwache Regionen wenig Aussichten auf eine Veränderung ihrer Situation. Lediglich im Bereich der Zweigniederlassungen, die jedoch in den letzten Jahren zurückgingen, konnten Ansiedlungen konstatiert werden. Zweigniederlassungen werden jedoch regional- und konjunkturpolitisch als problematisch eingestuft, da sie verhältnismäßig schnell geschlossen und eventuell auch wieder geöffnet werden, was den regionalen Arbeitsmarkt in den Peripherräumen nicht nachhaltig stabilisiert.170 "Noch ist zwar unsicher, ob durch die finanziellen Anreize im Rahmen der regionalen Wirtschaftsförderung diese Tendenz verstärkt wird - sicher ist aber, daß jedenfalls ein Brechen dieses Trends in den vergangenen 10 Jahren nicht erreicht werden konnte, so daß heute generell von einem Mißerfolg dieser Form von Förderung gesprochen wird."171

Die finanzielle Förderung wird somit nicht als geeignetes Instrument angesehen, um Betriebe, im sinne eines Pull-Effektes, zur Verlagerung aus den Verdichtungsräumen in periphere Räume zu motivieren. ${ }^{172}$ Hier dominieren die o.a. standortfaktoren, die $\mathrm{zu}$ einer verlagerung über eine mög-

$170 \mathrm{Vgl}$. Schliebe, K.; Hillesheim, D.: Das Standortverhalten neuerrichteter und verlagerter Industriebetriebe im Zeitraum von 1970 bis 1979, in: Informationen zur Raumentwicklung, Heft 11, 1980, S. 614.

171 Bullinger, D.: Tendenzen betrieblichen Standortwechsels in Ballungsräumen, a.a.0., S. 88 .

$172 \mathrm{Vgl.} \mathrm{exemplarisch} \mathrm{Gee,} \mathrm{C.} \mathrm{U.a.:} \mathrm{Infrastrukturelle} \mathrm{und}$ wirtschaftsstrukturelle Bestimmungsgründe der industriellen standortwahl, stuttgart 1980, S. $152 \mathrm{ff}$. 
lichst geringe Distanz führen. Zusätzlich werden standortverlagerungen, aufgrund der hohen Kosten, nur ungern vorgenommen. Der nahezu einzige Motivator für standortverlagerungen ist der Druck neue Flächen $z u$ suchen, weil sie am alten standort nicht mehr ausreichen (Push-Effekt). Werden zusätzlich die wenig mobilitätsbereite stammbelegschaft und der wunsch nach Erhaltung der Agglomerationsvorteile berücksichtigt, so ergeben sich mögliche Ansatzpunkte für eine Politik der Raumsteuerung.

Chancen ergeben sich im Bereich einer stärkeren Ausbildung einzelner Nebenkerne in einer polyzentrischen Großstadt-Umland-struktur, im sinne von dezentralen und eigenständigen Wohn-, Arbeits- und Versorgungsstandorten, die mittels der Raumsteuerungspolitik 173 realisiert werden könnten. Gefordert ist hier primär die Bauleit- und Regionalplanung, die durch die Regional-, Landschafts-, Verkehrs- und Flächennutzungspläne, langfristig gesehen, einen wesentlichen Einfluß auf die Gestaltung der verdichtungsräume nehmen kann. 174

\subsubsection{Zur Neuorientierung raumordnungspolitischer Hand- lungskonzepte}

Ein wesentliches Kennzeichen der gegenwärtigen Regionalpolitik ist die nahezu vollkommene Ausrichtung der Förderinstrumente auf die Investitionstätigkeit der privaten Unternehmen. Hierbei ist mit der Gewährung der Mittel, je nach Investitionsvorhaben, die Erfüllung bestimmter, beispiels-

173 Im Sinne von beispielsweise Verkehrs-, Grünflächenplanung, Gewerbe- und Wohnungspolitik.

$174 \mathrm{Vgl}$. Bullinger, D.: Tendenzen betrieblichen Standortwechsels in Ballungsräumen, a.a.0., S. 88 . 
weise arbeitsmarktbezogener Auflagen und ziele verbunden. 175

Hopfinger konnte im Rahmen einer empirischen studie nachweisen, daß der vermutete Zusammenhang zwischen der Vergabe von Investitionshilfen und der Entwicklung der Anzahl an Arbeitsplätzen in begünstigten Unternehmen in negativer Richtung verläuft. 176 Das bedeutet, daß mit zunehmender Förderungshäufigkeit eine quantitative Verschlechterung der Beschäftigungssituation einhergeht: "Die Zahl der Arbeitsplätze in den jeweiligen Betrieben entwickelt sich um so rückläufiger, je häufiger jene die angebotenen Förderhilfen in Anspruch nehmen können."177

Die Durchführung und Förderung strukturverbessernder Investitionsmaßnahmen ist, wie o.a., zum Schlüsselbereich der Regionalpolitik geworden und führt gleichzeitig zu einer Verschlechterung der Arbeitsbedingungen der Bevölkerung in den ausgewiesenen Problemgebieten. Dieses Dilemma motiviert Überlegungen bezüglich einer Neuorientierung der regionalen strukturpolitik, die im folgenden dargestellt werden.

Vor dem Hintergrund der veränderten Rahmenbedingungen behalten die grundlegenden zielvorstellungen der Raumordnung nach wie vor ihre Gültigkeit. Dagegen kommt es auf der Ebene der raumordnungspolitischen Handlungskonzepte zu einer Neuorientierung. Stichworte dazu sind: 'Die verstärkte Entwicklung des endogenen Potentials' und 'die Beseitigung regionaler Engpässe', wobei sich die Diskussion über die gegenwärtige Neuorientierung der regionalen strukturpolitik im wesentlichen um zwei Themenkomplexe dreht:

$175 \mathrm{Vgl}$. Hopfinger, H.: Für eine gespaltene Regionalpolitik unter veränderten Rahmenbedingungen, in: Raumforschung und Raumordnung, Heft 3, 1983, S. 91.

$176 \mathrm{Vgl}$. Hopfinger, H.: Für eine gespaltene Regionalpolitik unter veränderten Rahmenbedingungen, a.a.0., S. 96.

177 Ebenda. 
- die Neuabgrenzung der Fördergebiete 178 und

- die innovationsorientierte Regionalpolitik. 179

\section{Neuabgrenzung der Fördergebiete ${ }^{180}$}

Mit dem bestehenden Indikatorensystem werden die neuen arbeitsmarktpolitischen Problemregionen wie Bochum, Dortmund, Duisburg oder Gelsenkirchen nicht als Fördergebiete erkannt. Diese Regionen müssen momentan durch Sonderprogramme und Ausnahmeentscheidungen in das Fördergebiet mit einbezogen werden. Aus diesem Grund wird diskutiert, inwieweit verstärkt Arbeitsmarktaspekte zur Abgrenzung der Fördergebiete herangezogen werden sollen oder ob verschiedene Indikatoren, wie der Infrastrukturindikator, ganz gestrichen werden.

Provisorische Modellrechnungen zeigen, daß solche Vorschläge $z u$ Lasten des ländlichen Raums gehen. Die Arbeitslosenquote als entscheidender Abgrenzungsindikator würde zu einem abgehen von der zentralen Aufgabe der Regionalpolitik führen und die Gemeinschaftsaufgabe, die ein auf mittlere sicht angelegtes Instrument zum Abbau großräumiger regionalwirtschaftlicher Disparitäten darstellt, tendenziell zu einem Instrument des akuten Krisenmanagements des Arbeitsmarktes degenerieren.

Unter dem Aspekt eines Abbaus der Wohlstandsunterschiede können ländliche Gebiete, die immer noch Einkommens-, Wirtschaftsstruktur- und Infrakstrukturrückstände haben, nicht mit den Problemen der Agglomerationen gleichgesetzt werden, die zwar eine hohe Arbeitslosigkeit aber dennoch ver-

$178 \mathrm{Vgl}$. Buchner, W.: Neuorientierung der regionalen strukturpolitik, a.a.0., S. 169.

$179 \mathrm{Vg}$. Sternber, R: Technologie- und Gründerzentren als Instrument kommunaler Wirschaftsförderung, a.a.0., s. 48 .

$180 \mathrm{Vgl}$. Buchner, W.: Neuorientierung der regionalen Strukturpolitik, a.a.0., S. 169 f. 
gleichsweise eine hohe Wirtschaftskraft und günstige Wirtschaftsstruktur haben. Ein Verzicht der Indikatoren 'Infrastrukturausstattung' und/oder 'Einkommenssituation' ist deshalb aus ländlicher sicht nicht annehmbar. 181

\section{Innovationsorientierte Regionalpolitik}

Innovationsorientierte Regionalpolitik ist nach Brugger: "... eine regionalpolitisch motivierte und dezentral angelegte gesamtwirtschaftliche Innovationspolitik, die auf größere räumliche Chancengleicheit beim Zugang zum Innovationsproze $\beta$ und eine Korrektur raumbedeutsamer und -wirksamer gesamtwirtschaftlicher Vorkehrungen und Verhaltensweisen der Gemeinwesen abzielt"182. Sie sollte sich insbesondere auf drei Ziel- und Maßnahmenbereiche konzentrieren:

- die Vergrößerung des Innovationspotentials einer Region, 183

- die Verbesserung der regionalen Innovationsbedingungen 184 und

- die Motivierung innovativer Unternehmen zur quantitativen und qualitativen steigerung inrer Aktivitäten.185

$181 \mathrm{Vgl}$. ebenda.

182 Brugger, E.: Innovationsorientierte Regionalpolitik, in: Geographische Zeitschrift, Heft 3, 1980, S. 194 .

183 Im besonderen die Motivierung der Unternehmen, innerhalb und nicht außerhalb der Region zu investieren, die Anwerbung innovativer mobiler Unternehmen und die stimulierung der Gründung von technologieorientierten Unternehmensgründungen.

184 Insbesondere die Verfügbarkeit von Forschungs- und Entwicklungseinrichtungen und ein entsprechend qualifiziertes Arbeitskräftepotential.

$185 \mathrm{Vgl}$. Meyer-Krahmer, F.: Innovation Behaviour and Regional Indigenous Potential, in: Regional studies, Vol. 19, Heft 6, 1985, S. 532. 
Tabelle 2.3 stellt die grundsätzlichen Unterschiede zwischen der traditionellen mobilitätsorientierten Regionalpolitik und der innovationsorientierten Regionalpolitik dar.

Tabelle 2.3: Unterschiede zwischen mobilitätsorientierter Förderstrategie und innovationsorientierter Regionalpolitik ${ }^{186}$

\begin{tabular}{|c|c|c|}
\hline \multirow{2}{*}{$\begin{array}{l}\text { Unterscheidungs- } \\
\text { kriterium }\end{array}$} & \multicolumn{2}{|c|}{ Strategie } \\
\hline & $\begin{array}{l}\text { mobilitätsorientierte } \\
\text { Förderstrategie }\end{array}$ & $\begin{array}{l}\text { innovationsorientierte } \\
\text { Regionalpolitik }\end{array}$ \\
\hline $\begin{array}{l}\text { Engpaßfaktor- } \\
\text { hypothese }\end{array}$ & $\begin{array}{l}\text { Investitionslücke auf- } \\
\text { grund regionaler } \\
\text { Produktionskostennach- } \\
\text { teile }\end{array}$ & $\begin{array}{l}\text { Innovationslücke auf- } \\
\text { grund funktionaler } \\
\text { Defizite }\end{array}$ \\
\hline Zielgruppe & $\begin{array}{l}\text { Alle potentiellen } \\
\text { Investoren }\end{array}$ & $\begin{array}{l}\text { 'Endogene' Betriebe und } \\
\text { Unternehmen der Förder- }\end{array}$ \\
\hline Ansatzpunkte & $\begin{array}{l}\text { Investitionskosten der } \\
\text { Unternehmen }\end{array}$ & $\begin{array}{l}\text { Handlungspotential der } \\
\text { Unternehmen }\end{array}$ \\
\hline Instrumente & $\begin{array}{l}\text { Globale finanzielle } \\
\text { Investitionsanreize } \\
\text { und Ausbau der wirt- } \\
\text { schaftsnahen Infra- } \\
\text { struktur }\end{array}$ & $\begin{array}{l}\text { Selektive finanzielle } \\
\text { Anreize zum Aufbau an- } \\
\text { passungswichtiger Funk- } \\
\text { tionen und Beratungs- } \\
\text { instrumente und geziel- } \\
\text { tes externes Angebot } \\
\text { managementorientierter } \\
\text { Serviceleistungen }\end{array}$ \\
\hline Wirkungsweise & $\begin{array}{l}\text { Veränderung der wett- } \\
\text { bewerbsbedingungen } \\
\text { zugunsten der Förder- } \\
\text { gebiete und daraus } \\
\text { folgende Nachfrage- } \\
\text { verlagerung }\end{array}$ & $\begin{array}{l}\text { Veränderung des Wett- } \\
\text { bewerbspotentials der } \\
\text { Fördergebiete; daraus } \\
\text { folgend Nachfrageum- } \\
\text { lenkung und Neu-Kre- } \\
\text { ation von Nachfrage }\end{array}$ \\
\hline Wirkungsdauer & Eher kurzfristig & Eher langfristig \\
\hline $\begin{array}{l}\text { Komplexitäts- } \\
\text { grad }\end{array}$ & Relativ niedrig & Relativ hoch \\
\hline
\end{tabular}

186 In Anlehnung an Sternber, R: Technologie- und Gründerzentren als Instrument kommunaler Wirschaftsförderung, a.a.O., S. 49 . 
In der Diskussion über die innovationsorientierte Regionalpolitik besteht weitgehend konsens, daß die konkrete regionale Anwendung ihrer allgemeinen Grundsätze derzeit noch im experimentell geprägten Anfangsstadium ist. 187 Hierbei existieren bereits heute Befürchtungen, daß eine gesamtwirtschaftliche Innovationspolitik, die periphere Regionen auch, aber nicht explizit, fördert, die regionalen Ungleichgewichte kaum beseitigen wird.

Die Beurteilung der momentan existierenden Telekommunikationsförderungen, die in Kapitel 2.3 dargestellt wurden, wird im Anschluß an die folgende Darstellung der empirischen Erhebung vogenommen, in der eine Analyse der Bewertung von Fördermaßnahmen durch kleine- und mittlere Unternehmen enthalten ist.

$187 \mathrm{Vgl}$ : Stöhr, W.: Ansätze zu einer neuen Fundierung der Regionalpolitik, a.a.0., S. 11; Meyer-Krahmer, F.: Innovation Behaviour and Regional Indigenous Potential, a.a.0., s. 533 . 
3. Einzelbetriebliche Untersuchung des Einflusses der Telematik auf die standortentscheidung von klein- und Mittelbetrieben

\subsection{Rennzeichnung der Untersuchung}

\subsubsection{Grundlagen und ziele}

\subsubsection{Untersuchungsansatz}

In der wissenschaftlichen Diskussion über die neuen Informations- und Telekommunikationstechniken besteht weitgehend Einigkeit darüber, daß Technische Entwicklung, Ausbau der Kommunikationsinfrastruktur, Adoptionsentwicklung und Wirtschaftsentwicklung einer Region interdependent sind. 1

Ausgehend vom ISDN-Konzept ${ }^{2}$ der Deutschen Bundespost, sollen Technik, Netze, Dienste und Endgeräte integriert werden, wobei neben den grundsätzlich neuen Diensten, die option für eine simultane Verwendung verschiedener Dienste besteht. Im Bereich der Endgeräte wird es zu Neuentwicklungen von multifunktionalen und Mehrdienste-Endgeräten kommen. Diese implizieren ein erhebliches Veränderungspotential für die Gesamtwirtschaft. Neben den erwarteten syner-

$1 \mathrm{Vgl}$. Lange, S.: Telematik und regionale Wirtschaftspolitik, a.a.O., Schön, H.: ISDN und ökonomie, a.a.O., S. 950, Spehl, H.: Räumliche Wirkungen der Telematik, a.a.0., S. $254 \mathrm{ff}$. und Tenzer, G. (Hrsg.): Büroorganisation - Bürokommunikation, Mittel zur steigerung der Produktivität, Heidelberg, 1984.

2 Vgl. Deutsche Bundespost: ISDN - die Antwort der Deutschen Bundespost auf die Anforderungen der Telekommunikation von morgen, Bonn 1984 und oberpostdirektion Frankfurt: Leitfaden Telekommunikation, Band 1, Frankfurt 1986 . 
gieeffekten, wird es zu temporär differenzierten neuen Anwendungsentwicklungen in Unternehmen, öffentlichen Verwaltungen und Haushalten kommen. Diese neuen Anwendungen verursachen erhebliche innere strukturveränderungen, die momentan nicht abzuschätzen sind.

Aus dieser Perspektive heraus wird die wirtschaftspolitische Relevanz der gesamten Netz- und Diensteausbauplanung der Deutschen Bundespost evident. Die Deutsche Bundespost setzt faktisch die Restriktionen und Standards für Hersteller und Anwender und determiniert weitgehend, durch das Netz- und Diensteangebot, das Kommunikationspotential in der Bundesrepublik Deutschland. ${ }^{3}$

Kenntnisse über den Stand und die Adoption der neuen telekommunikativen Techniken sind deshalb unerläßlich, um Ansatzpunkte für ein regionalpolitisches Handeln zu finden.

Die bereits existierenden studien für die Bundesländer Nordrhein-Westfalen ${ }^{4}$, Niedersachsen, Rheinland-Pfalz ${ }^{5}$ und Hessen ${ }^{6}$ und die regionalen empirischen Untersuchungen für z.B. Hamburg ${ }^{7}$, Ostbayern ${ }^{8}$, Mühlheim/Ruhr ${ }^{9}$ und $\operatorname{Trier}^{10}$ ana-

3 Vgl. Winand, U. U.a.: Telekommunikation in Hessen, a.a.o., s. 1 f.

4 Vgl. Scientific Control System GmbH (Hrsg.): Studie für einen 'Telekommunikationsentwicklungsplan' für das Land Nordrhein-Westfalen, Essen 1984.

5 Vgl. Scharlach, C. u.a.: Aspekte der Telekommunikationsentwicklung im Land Rheinland-Pfalz, a.a.o..

6 Vgl. Winand, U. u.a.: Telekommunikation in Hessen, a.a.o..

7 Vgl. Lange, S. u.a.: Lokale Initiativen zur Förderung der Anwendung der Telekommunikationstechniken, Telematik und regionale Wirtschaftspolitik, Band II, Köln 1986.

8 Vgl. Haldenwang, H.: Anwendungen neuer Informations- und Kommunikationstechniken in Ostbayern, in: Spehl, H.; Messerig-Funk, B. (Hrsg.): Telematik Trier, Ergebnis eines symposiums, Beiträge aus Forschung und Praxis, Band I, Trier 1986, S. 62-90. 
lysieren Teilaspekte, geben jedoch keinen Aufschluß bezüglich der hier untersuchten Fragenkomplexe ${ }^{11}$. Die Untersuchung in Hessen konzentrierte sich lediglich auf den zukünftigen Telekommunikationsbedarf.

Motivation für die empirsche Erhebung des Verfassers war, neben der Differenzierung der empirischen Untersuchung nach unterschiedlichen Regionstypen, bezüglich siedlungsstruktur und Industrialisierung in Hessen, die Erfassung der bisher nur ungenügend berücksichtigten Gruppen der Klein- und Mittelbetriebe und der neugegründeten Unternehmen. Weiterer Bestandteil der Untersuchung des Verfassers ist die empirische Erhebungen bezüglich der Telekommunikationsverfügbarkeit als Standortfaktor und bezüglich des Adoptionsverhaltens gegenüber den Telekommunikationsförderungsmöglichkeiten, worüber momentan keine empirischen Erhebungen für den Untersuchungsraum vorliegen.

\subsubsection{Arbeitshypothese und abgeleitete Unterhypothesen}

Die regionalpolitischen Aktivitäten zur Förderung von strukturschwachen Regionen könnten unter anderem eine verstärkte Förderung neuer telekommunikativer Techniken umfassen. Dies ist aber nur dann sinnvoll, wenn der Aspekt neue telekommunikative Techniken mitentscheidend bei der stand-

9 Vgl. Tiemann, U.: Anwendungen neuer Informations- und Kommunikationstechnologien im Ruhrgebiet, in: Spehl, H.; Messerig-Funk, B. (Hrsg.): Telematik Trier, Ergebnis eines Symposiums, Beiträge aus Forschung und Praxis, Band I, a.a.0., S. 91-104.

$10 \mathrm{Vgl}$. Spehl, H.; Messerig-Funk, B.: Chancen und Probleme der wirtschaftlichen Nutzung der Telematik in einer ländlichen Region: Trier, in: Akademie für Raumforschung und Landesplanung (Hrsg.): Räumliche Wirkungen der Telematik, Hannover 1987, S. 417-445.

11 Siehe Kapitel 3.1.1.3.. 
ortwahl ist. Daher bildet folgende Arbeitshypothese die Grundlage des Untersuchungskonzeptes.

\section{Telekommunikation ist ein standortfaktor für klein- und kittelbetriebe}

Hierbei ist im besonderen der Einfluß von Telekommunikationsfördermaßnahmen zu berücksichtigen.

Weitere Hypothesen, die mit der Arbeitshypothese in Zusammenhang stehen:

1. Bei der Adoption neuer telekommunikativer Techniken existiert ein Gefälle zwischen den Agglomerationen und den strukturschwachen Gebieten. 12

2. Die Charakteristika der standorte haben einen substantiellen Einfluß auf das qualitative Adoptionsverhalten der Anwender. 13

3. Zwischen der Größe eines Unternehmens und seinem Adoptionsverhalten existiert ein signifikanter Zusammenhang. Größere Unternehmen sind typische Frühadoptoren, wogegen kleinere Unternehmen zu den Spätadoptoren $z a ̈ h-$ len. 14

4. Das Vorhandensein von Forschungs- und Entwicklungsaktivitäten fördert die Adoption neuer Telekommunikationstechniken.

5. Selbständige Einzelbetriebe weisen gegenüber Unterneh-

12 Nachfrageorientiert rein quantitativ.

13 Qualitativ im Sinne von unterschiedlichen Diensten.

14 Die Investitions- und Betriebskosten der Telekommunikationsdienste haben einen evidenten Einfluß in Bezug auf das temporäre, quantitative und qualitative Adoptionsverhalten. 
men mit mehreren Betriebsstätten eine geringere Adoptionsrate bezüglich der Telekommunikationsdienste auf.

6. Der Bedarf an speziellen Telekommunikationsdiensten ist nicht homogen. Der nachgefragte Dienst resultiert primär aus dem Kommunikationsverhalten der jeweiligen Branche.

7. Die Nachfrage nach neuen telekommunikativen Diensten wird nicht durch die reine Verfügbarkeit der Dienste ausgelöst, sondern durch die Kenntnis möglicher Anwendungen und Kosten.

8. Mikro- bzw. Personal-Computer-Anwender zählen zu den Frühadoptoren.

Mit der empirischen Untersuchung wurde die Arbeisthypothese einschließlich der o.a. abgeleiteten Hypothesen überprüft.

\subsubsection{Zielsetzung der Erhebung}

Ausgehend vom finanzwissenschaftlichen, traditionellen Steuerungsinstrumentarium ist, als Hauptziel der Untersuchung, zu überprüfen, ob die neuen telekommunikativen Techniken als regionalpolitisches steuerungsinstrument verwendet werden können. Insbesondere stellt sich die Frage, ob eine Förderung der neuen telekommunikativen Techniken, unter der Prämisse einer regionalpolitischen zielsetzung, erforderlich ist und wenn $j a$, in welcher Art.

Im speziellen sollen folgende Fragenkomplexe erörtert werden:

- Stellen die neuen Telekommunikationstechniken, beziehungsweise ihre Verfügbarkeit, einen neuen standortfaktor 
dar?

- Existiert ein Adoptions-Nord-Süd-Gefälle innerhalb Hessens?

- Existieren regionstypenbedingte Adoptions-Disparitäten bezüglich der neuen Telekommunikationstechniken?

- Welche Telekommunikationsstruktur liegt in den zu untersuchenden Regionen in Hessen vor?

- Was sind die Ursachen und Gründe der Rejektion im Bereich der Adoption neuer telekommunikativer Techniken?

- Wie stellt sich das Adoptionsverhalten bezüglich der Telekommunikationsfördermöglichkeiten für die zu untersuchenden Gruppen dar?

\subsubsection{Konzept und Realisierung der Erhebung}

\subsubsection{Auswahl und Abgrenzung der Unternehmen und Unter- suchungsgebiete}

\section{Auswahl und Abgrenzung der Unternehmen}

Die Auswahl und Abgrenzung der Unternehmen bezüglich ihrer Branchenzugehörigkeit wurde in Anlehnung an die systematik der wirtschaftszweige des statistischen Bundesamtes vorgenommen.

Bei der Auswahl wurden die Branchen berücksichtigt, deren vermuteter Kenntnis- und Erfahrungsstand es rechtfertigt, Aussagen über neue telekommunikative Techniken zu treffen. Eine Branche, deren vermuteter Kenntnis- und Erfahrungs- 
stand erheblich unter dem der drei anderen Gruppen liegt, wurde als Vergleichsgruppe gewählt.

Aufgrund der zielsetzung der Gemeinschaftsaufgabe 'Verbesserung der regionalen Wirtschaftsstruktur', primär überregional arbeitende Unternehmen zu fördern (Export-BasisTheorie), 15 wurden aus dem Bereich Private Dienstleistungen die folgenden Branchen ausgewählt:

Unternehmensberatungen und software-Firmen bzw. EDV-Vertrieb.

Aus dem Bereich Verarbeitendes Gewerbe wurde die Branche

Maschinenbau

und als Vergleichsgruppe zu diesen Branchen der

\section{Einzelhandel}

gewählt.

Die Untersuchung beschränkt sich im wesentlichen auf Kleinund Mittelbetriebe, die gegenwärtig die Hauptadressaten der existierenden Programme zur Förderung der Einführung und Anwendung neuer telekommunikativer Techniken darstellen. 16

$15 \mathrm{Vgl}$. Deutscher Bundestag: Siebzehnter Rahmenplan der Gemeinschaftsaufgabe 'Verbesserung der regionalen Wirtschaftsstruktur', a.a.O., S. 28 und Rittenbruch, K.: Zur Anwendbarkeit der Exportbasis-Konzepte im Rahmen von Regionalstudien, Schriften zu Regional- und Verkehrsproblemen in Industrie- und Entwicklungsländern, Band 4, Berlin 1968 .

16 Siehe Kapitel 'Förderungsmöglichkeiten telekommunikativer Techniken'. 


\section{Auswahl und Abgrenzung der Untersuchungsgebiete}

Die Auswahl und Abgrenzung der auf Hessen beschränkten Untersuchungsgebiete wurde anhand des Indikatorensystems der Institution Gemeinschaftsaufgabe 'Verbesserung der regionalen Wirtschaftsstruktur' durchgeführt. Die gesetzliche Grundlage der Gemeinschaftsaufgabe bildet das Gesetz über die Gemeinschaftsaufgabe vom 6.10.1969, das in $\$ 1$ festlegt, daß alljene Gebiete innerhalb der Bundesrepublik Deutschland zu fördern sind,

"1. deren Wirtschaftskraft erheblich unter dem Bundesdurchschnitt liegt oder erheblich darunter abzusinken droht oder

2. in denen Wirtschaftszweige vorherrschen, die vom strukturwandel in einer Weise betroffen oder bedroht sind, daß negative Rückwirkungen auf das Gebiet in erheblichem Umfang eingetreten oder absehbar sind". 17

Bundesweit werden 179 regionale Arbeitsmärkte unterschieden und auf je fünf Einzelindikatoren hin untersucht. Dies sind

- die durchschnittliche Arbeitslosenquote der Jahre 19811985 (ALQ 1981-85),

- die Bruttowertschöpfung des Jahres 1982 zu Faktorkosten je Einwohner (BWS 1982),

- die Bruttolohn- und Bruttogehaltssumme des Jahres 1982 je Beschäftigten (L+G 1982),

- die Prognose der Arbeitsmarktbilanz 1990 (Arbeitskräftereservekoeffizient) und

- die Infrastrukturausstattung (Infra 1982) 18.19

17 Gesetz über die Gemeinschaftsaufgabe 'Verbesserung der regionalen Wirtschaftsstruktur' vom 6. Oktober 1969, BGBl. I, S. 1861 .

18 Der Infrastrukturindikator wird hier in Verkehr, Energie, Soziales, Ausbildung und Umwelt unterteilt.

$19 \mathrm{Vgl}$. Der Hessische Minister für Wirtschaft und Technik: Jahresbericht 1986, Frankfurt 1987, S. 23. 
Bei der Zusammenfassung der Einzelindikatoren zu einem Gesamtindikator erfolgt eine Normierung auf einen Durchschnittswert von 100 und eine standardabweichung von 15.20 Die Bruttowertschöpfung und die Bruttolohn- und Bruttogehaltssumme werden mit jeweils 0.225 gewichtet, die Arbeitslosenquote mit 0.45 und die Infrastrukturausstattung mit 0,1 . Der Arbeitskräftereservekoeffizient wurde in den Gesamtindikator nicht mit eingerechnet. 21

Zusätzlich zur Indikatorenzuordnung werden die Regionen nach Schwerpunktorten untergliedert, in denen unterschiedliche Förderungshöchstsätze gelten. Dies sind

- die übergeordneten Schwerpunkte im Zonenrandgebiet (ASchwerpunkte mit maximal 23\% Förderung),

- die übergeordneten Schwerpunkte außerhalb des Zonenrandgebietes (B-Schwerpunkte mit maximal 18\% Förderung),

- die sonstigen Schwerpunkte (C-Schwerpunkte mit maximal 15\% Förderung) sowie

- die Schwerpunkte in extremer Zonenrandlage (E-Schwerpunkte mit maximal 23\% Förderung). 22

In Tabelle 3.1 werden die Ausprägungen der Förderindikatoren und des Gesamtindikators, im Rahmen der Gemeinschaftsaufgabe 'Verbesserung der regionalen wirtschaftsstruktur', dargestellt. Ausgewählt wurden vier Regionen, die durch Fettschrift und kursive Darstellung hervorgehoben sind. Die Auswahl der Regionen wurde unter dem Aspekt vorgenommen,

$20 \mathrm{Vgl.}$ Eckey, H. U.a.: Sozioökonomische und ökologische Bedingungen für die Entwicklung Nordhessens, a.a.o., s. 160 .

21 Vgl. Der Hessische Minister für Wirtschaft und Technik: Jahresbericht 1986, a.a.O. S. 22 f.

$22 \mathrm{Vgl}$. Deutscher Bundestag: Siebzehnter Rahmenplan der Gemeinschaftsaufgabe 'Verbesserung der regionalen wirtschaftsstruktur', a.a.0., s. 29 . 
daß den ausgewählten 'Normalgebieten'23 je ein entsprechendes Fördergebiet gegenübergestellt werden soll.

Tabelle 3.1: Einzel- und Gesamtindikatoren der hessischen Arbeitsmarktregionen ${ }^{24}$

\begin{tabular}{|c|c|c|c|c|c|c|}
\hline $\mathbf{N r}$. & $\begin{array}{l}\text { Regionaler Ar- } \\
\text { beitsmarkt }\end{array}$ & $\begin{array}{c}A L Q \\
1981-85\end{array}$ & $\begin{array}{l}\text { BWS }^{25} \\
1982\end{array}$ & $\begin{array}{c}L+G^{26} \\
1982\end{array}$ & $\begin{array}{l}\text { Infra } \\
1982\end{array}$ & $\begin{array}{l}\text { Gesamt- } \\
\text { indik. }\end{array}$ \\
\hline 066 & Kassel & 10,80 & 231,99 & 321,93 & 106,93 & 94,13 \\
\hline 082 & Korbach & 9,20 & 194,34 & 293,30 & 93,84 & 90,93 \\
\hline 083 & Eschwege & 10,30 & 166,62 & 293,22 & 98,28 & 86,93 \\
\hline 084 & Hersfeld & 8,50 & 193,18 & 305,07 & 93,48 & 94,10 \\
\hline 085 & $\begin{array}{l}\text { Alsfeld-ziegen- } \\
\text { hain }\end{array}$ & 9,60 & 185,52 & 294,40 & 75,16 & 87,69 \\
\hline 086 & $\begin{array}{l}\text { Marburg-Bieden- } \\
\text { kopf }\end{array}$ & 7,50 & 172,21 & 303,44 & 106,79 & 96,44 \\
\hline 087 & Dillenburg & 8,40 & 211,43 & 312,68 & 112,29 & 98,31 \\
\hline 092 & $\begin{array}{l}\text { Limburg-Westerw. } \\
\text {-Lahn }\end{array}$ & 7,60 & 150,51 & 290,43 & 84,17 & 90,95 \\
\hline 093 & Gießen & 8,10 & 213,49 & 312,66 & 112,29 & 99,19 \\
\hline 094 & Fulda & 8,40 & 191,02 & 291,26 & 99,68 & 93,10 \\
\hline 095 & $\begin{array}{l}\text { Gelnhausen- } \\
\text { Schlüchtern }\end{array}$ & 7,00 & 153,87 & 289,48 & 86,57 & 92,79 \\
\hline 096 & Prankfurt & 5,80 & 368,99 & 369,82 & 129,71 & 123,48 \\
\hline 097 & Wiesbaden-Mainz & 6,10 & 299,61 & 348,45 & 119,90 & 114,78 \\
\hline 106 & Darmstadt & 5,40 & 234,40 & 322,46 & 116,29 & 110,14 \\
\hline
\end{tabular}

23 Wobei als 'Normalgebiete' der in Hessen wirtschaftlich dominierende Verdichtungsraum Frankfurt für Südhessen und Gießen, als ländlicher Raum mit Verdichtungskernen, für Mittelhessen ausgewählt wurden.

24 In Anlehnung an Eckey, H. U.a.: Sozioökonomische und ökologische Bedingungen für die Entwicklung Nordhessens, a.a.0., S. 161-162.

25 Einwohner (in 100 DM)

26 Arbeitnehmer (in $100 \mathrm{DM}$ ) 
Die ausgewählten Regionen sind:

1. GroBraum Frankfurt

- Normalgebiet

- Verdichtungsraum 28

- Gesamtindikator $=123,48$
2. Großraum Kassel

- Fördergebiet 27

- Verdichtungsraum

- Gesamtindikator $=94,13$
3. Großraum Gießen

- Normalgebiet

- ländlicher Raum mit Verdichtungskernen

- Gesamtindikator $=99,19$
4. Großraum Fulda

- Fördergebiet

- ländlicher Raum mit Verdichtungskernen

- Gesamtindikator $=93,10$

Aus den in Tabelle 3.1 dargestellten Gesamtindikatoren resultieren die Fördergebiete ${ }^{29}$ des 17 . Rahmenplans der Gemeinschaftsaufgabe 'Verbesserung der regionalen wirtschaftsstruktur' in Hessen, die nicht per se durch ihre Zonenrandlage als Fördergebiete ausgewiesen werden. Die Fördergebiete werden weiterhin ergänzt "durch noch nicht berücksichtigte Arbeitsmarktregionen, sofern deren Arbeitslosenquote im Durchschnitt der Jahre 1981 bis 1985 über 160\% des Bundesdurchschnitts beträgt" 30 .

Folgende Abbildung 3.1 stellt, für den Bereich Hessen, das 'Zonenrand- und Fördergebiet der Gemeinschaftsaufgabe', das 'Fördergebiet der Gemeinschaftsaufgabe' und die 'Gebiete des Hessischen Strukturförderungsprogramms' dar.

27 Wobei die Fördergebiete Kassel und Fulda aufgrund ihrer wirtschaftsräumlich peripheren Lage und der Grenznähe zur Deutschen Demokratischen Republik, als A-Schwerpunktorte eingestuft sind.

28 Eie Klassifizierung nach Regionstypen erfolgte in Anlehnung an die Einteilung der HLT Gesellschaft für Forschung Planung Entwicklung mbH. Stand: Wiesbaden 1983.

29 Dies geschieht durch den Vergleich des jeweiligen Gesamtindikators mit einem schwellenwert.

30 Hessisches Ministerium für Wirtschaft und Technik: Konjunkturbericht August 1986, Wiesbaden 1986, S. 52 . 
Abbildung 3.1: Fördergebiet der Gemeinschaftsaugabe 'Verbesserung der regionalen wirtschaftsstruktur' des 17. Rahmenplanes ${ }^{31}$

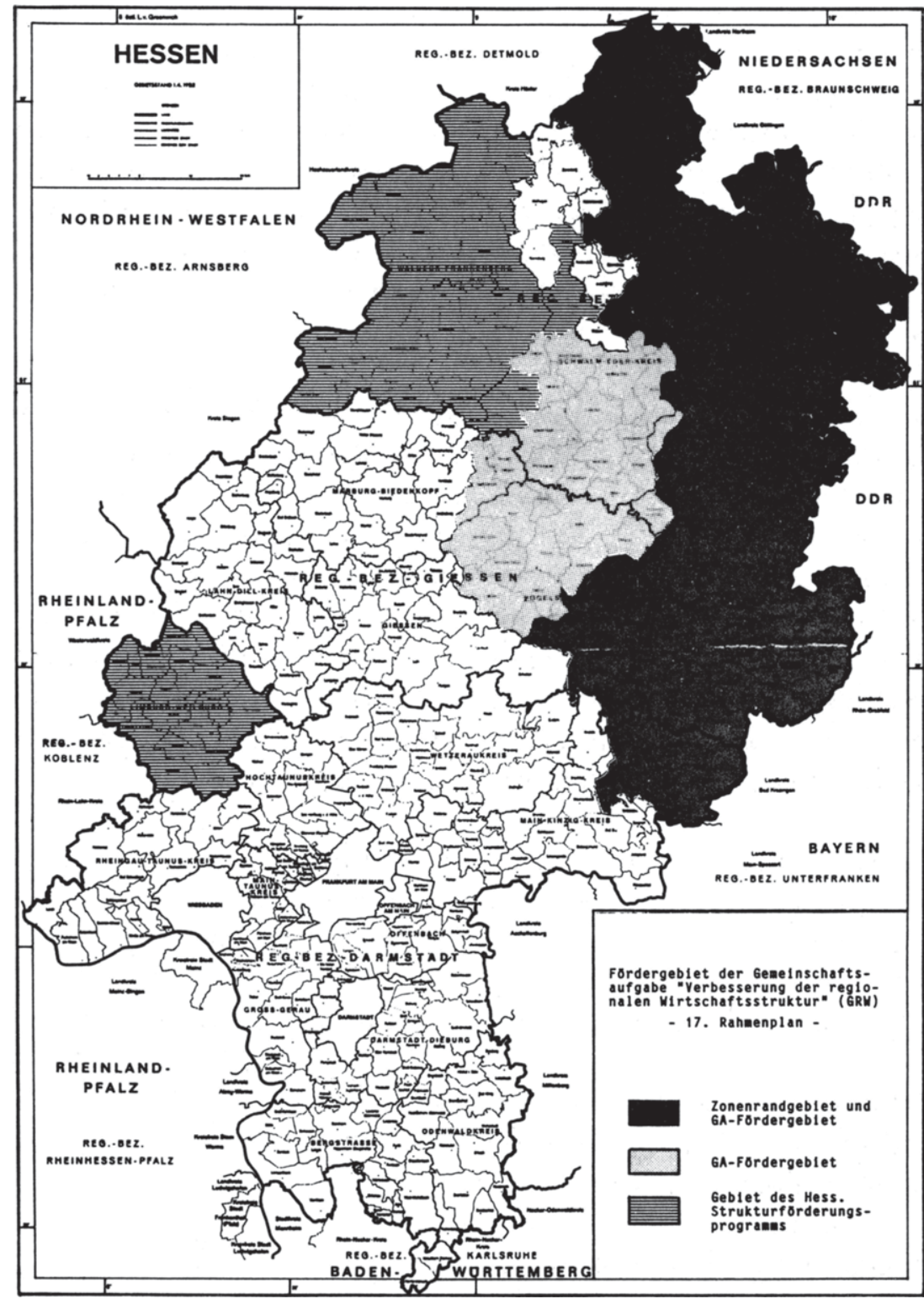

31 Quelle: Hessisches Ministerium für Wirtschaft und Technik: Jahresbericht 1988, Wiesbaden 1989, S. 28. 


\subsubsection{Erhebungsmethodik}

Die Untersuchung beschränkt sich, wie o.a., auf das Land Hessen und hier auf den Ballungsraum Frankfurt, die altindustrialisierte Agglomeration Kassel, den Raum Gießen und das Zonenrandfördergebiet Fulda. Es wurde der Bereich des 'Geschäftsbedarfs' der Individualkommunikation mit einem empirischen Untersuchungskonzept analysiert. Als Explorationsinstrument wurde eine Fragebogenaktion ausgewählt, die im wesentlichen auf Klein- und Mittelbetriebe zugeschnitten ist.

Das in der empirischen Forschung herrschende Postulat einer statistischen Repräsentativität der Stichprobe wurde zugunsten einer 'informatorischen' Repräsentativität aufgegeben. Drei Branchen wurden abgefragt, deren vermuteter Kenntnisund Erfahrungsstand es rechtfertigt, Aussagen über neue telekommunikative Techniken $z u$ treffen, sodaß systematische Fehler aufgrund unzureichender Informationen tendenziell vermieden wurden. Eine vierte Branche, deren vermuteter Kenntnis- und Erfahrungsstand erheblich unter dem der drei anderen Gruppen liegt, diente als Kontrollgruppe.

Basis der Frageninhalte ist die in Kapitel 3.1.1.2. aufgestellte Arbeitshypothese und die korrespondierenden Unterhypothesen.

Der Fragebogen gliedert sich in folgende Themenkomplexe:

- allgemeine strukturelle Fragen,

- Kenntnis- und Adoptionsstand,

- Adoptionshemmisse,

- Standorteinschätzung und -verhalten,

- Telekommunikationsfördermaßnahmen,

- Kommunikationsverhalten und

- Substitutionspotential persönlicher Kontakte durch 
neue telekommunikative Techniken.

Die allgemeinen strukturellen Fragen umfassen:

- die regionale Zuordnung,

- die Betriebsgröße,

- den Umsatz,

- die Branchenzugehörigkeit,

- die Anzahl der Betriebsstätten,

- die Mikro- bzw. Personal-Computer-Anwendung,

- die Forschungs- und Entwicklungsaktivitäten,

- die Fernmeldegebühren und Endgerätekosten und

- das Gründungsjahr des Unternehmens.

Diese allgemeinen betrieblichen strukturdaten werden im folgenden als Determinanten der Telekommunikationsentwicklung überprüft, indem bei der Auswertung entsprechende Signifikanzprüfungen vorgenommen werden.

\subsubsection{3. stichprobenumfang, Rücklauf und Repräsentanz}

Zur Vorbereitung der Haupterhebung wurde ein Pre-Test bei ausgewählten Firmen durchgeführt, der eine Antwortquote von 73\% hatte. Die Vorerhebung diente schwerpunktmäßig der Überprüfung der Verständlichkeit der gestellten Fragen.

Mit der Haupterhebung wurden 772 Unternehmen erreicht. Ihre Adressen wurden per Zufallsauswahl aus wenigen, direkt zugänglichen Quellen ${ }^{32}$ gewonnen.

Die Versendung der Fragebögen fand im Frühjahr 1989 statt. Der bis Ende August 1989 abgeschlossene Rücklauf enthielt

32 Aus finanziellen Gründen erfolgte eine Beschränkung auf direkt zugängliche Quellen wie 'Wer gehört zu wem,...' u.ä.. 
137 Antworten, was einer Rücklaufquote von $18 \%$ entspricht. 33 Die angegebenen Gründe bei Nichtbeantwortung des Fragebogens waren vor allem Zeit- und Personalmangel sowie mangelnde Repräsentativität für die entsprechende Branche bzw. Region.

Aufgrund der nach Regionen und Unternehmensgrößen vorgenommenen Selektion der stichprobe, darf diese nicht als repräsentativ für die Grundgesamtheit der Unternehmen in der Bundesrepublik betrachtet werden. Es "kann nicht ausgeschlossen werden, daß die Unternehmen, die den Fragebogen nicht beantwortet haben, signifikant anders als die antwortbereiten Firmen" 34 handeln.

Aufgrund der vorliegenden Ergebnisse, ist darauf hinzuweisen, daß die Unternehmen tendenziell in den einzelnen sektoren überrepräsentiert sind, die die neuen telekommunikativen Techniken bereits adoptiert haben.

\subsubsection{8tatistische Auswertungsverfahren}

Im Rahmen der Erhebung wurden insgesamt 23 Fragen mit weitgehend vorgegebenen Antwortmöglichkeiten gestellt. Bezüglich des Meßniveaus liegen im Bereich der betriebsstrukturellen Fragen zum großen Teil nominal skalierte Daten vor, wohingegen die restlichen Fragen größtenteils ordinal skaliert sind. Die Auswertung wurde mit dem statistikprogramm STATGRAPHICS vorgenommen und durch HARVARD GRAPHICS im Be-

33 Ein Teil der Antworten ging erst nach einem Erinnerungsschreiben ein.

34 Schanz, G.: Kriterien zur Bestimmung der Größe des Forschungsbudgets in Unternehmungen der Industriegruppe Elektrotechnik, in: Zeitschift für betriebswirtschaftliche Forschung, 2, 1972, S. 81. 
Tabelle 3.2: Verwendete statistische Verfahren ${ }^{35}$

\begin{tabular}{|c|c|c|c|}
\hline \multirow[b]{2}{*}{ Meßniveau } & \multicolumn{2}{|c|}{ Nichtparametrische statistische Tests } & \multirow{2}{*}{$\begin{array}{l}\text { Nichtparametrische } \\
\text { Korrelationsmaße }\end{array}$} \\
\hline & $\begin{array}{l}\text { Der Fall zweier unab- } \\
\text { hångiger Stichproben }\end{array}$ & $\begin{array}{l}\text { Der Fall mit k unab- } \\
\text { hangigen Stichproben }\end{array}$ & \\
\hline \multirow{2}{*}{ Nominal } & $\begin{array}{l}\text { Fisher-Test }(2 \times 2) \\
\text { (tar kleine stichproben) }\end{array}$ & \multirow{2}{*}{$\begin{array}{l}x^{2}-\text { Test } \quad(k \times r) \\
\text { (for grope stichproben })\end{array}$} & $\begin{array}{l}\text { Kontingenz- } \\
\text { Koeffizient C }\end{array}$ \\
\hline & $\begin{array}{l}x^{2}-\text { Test } \quad \text { (2x2) } \\
\text { (for gropo stichproben) }\end{array}$ & & Cramer's V \\
\hline \multirow{2}{*}{ Ordinal } & $\begin{array}{l}\text { Mann - Whitney - U - Test } \\
\text { (tar grope stichprobon) }\end{array}$ & \multirow{2}{*}{$\begin{array}{l}\text { Ein - Weg-Rangvarianz- } \\
\text { analyse nach Kruskal } \\
\text { und Wallis ( H-Test ) }\end{array}$} & $\begin{array}{l}\text { Rang-Korrelations- } \\
\text { Koeffizient r nach } \\
\text { Spearman }\end{array}$ \\
\hline & $\begin{array}{l}\text { Kolmogorov-Smirnov-Test } \\
\text { (for gropo stichproben) }\end{array}$ & & $\begin{array}{l}\text { Rang-Korrelations- } \\
\text { Koeffizient Tau nach } \\
\text { Kendall }\end{array}$ \\
\hline
\end{tabular}

35 In Anlehnung an Siegel, S.: Nichtparametrische statistische Methoden, 2. Auflage, Eschborn 1985 und Sachs, L.: Angewandte Statistik - Statistische Methoden und ihre Anwendungen, 5. Auflage, Berlin, Heidelberg, New York 1978 . 
reich der grafischen Darstellung ergänzt. Die verwendeten statistischen Verfahren sind in Tabelle 3.2 dargestellt.

Aufgrund der vorliegenden nominal und ordinal skalierten Daten, konnten bei der Auswertung deskriptive und inferenzstatistische verfahren angewandt werden. ${ }^{36}$ Die erhobenen und klassifizierten Daten wurden auf statistisch signifikante Zusammenhänge und die Intensität dieser zusammenhänge überprüft.

Die am häufigsten verwendeten inferenzstatistischen Verfahren waren die "Ein-Weg-Rangvarianzanalyse nach Kruskal und Wallis"37 und der "Mann-Whitney-U-Test"38. Der Einsatz multivariater Verfahren ist, aufgrund des Meßniveaus der Daten, nicht möglich. 39

Die Kennzeichung der Wahrscheinlichkeit, mit der ein dargestellter Zusammenhang sich nicht zufällig ergeben hat, wird im folgenden durch das Signifikanzniveau dargestellt. Die Interpretation des jeweiligen signifikanzniveaus wurde anhand von Tabelle 3.3 vorgenommen.

36 Vgl. Bamberg, G.; Baur, F.: Statistik, München, Wien 1980 , S. 133 ff und Menges, G.: Die Statistik - Zwölf Stationen des statistischen Arbeitens, Wiesbaden 1982, S. $263 \mathrm{ff}$.

37 Vgl. Siegel, S.: Nichtparametrische statistische Methoden, 2. Auflage, a.a.0., S. 176-184 und STSC, Inc.: STATGRAPHICS - USER'S GUIDE, Maryland, Rockville 1988, S. 14.15 .

$38 \mathrm{Vgl}$. Siegel, S.: Nichtparametrische statistische Methoden, a.a.O., S. 112-123 und STSC, Inc.: STATGRAPHICS USER'S GUIDE, a.a.o., s. 22.10 .

$39 \mathrm{Vgl.:}$ Backhaus, K. u.a.: Multivariate Analysemethoden Eine anwendungsorientierte Einführung, 4. Auflage, Heidelberg u.a. 1987, S. XI ff. 
Tabelle 3.3: Kennzeichung des Signifikanzniveaus 40

\begin{tabular}{|r|r|l|}
\hline $\begin{array}{c}\text { Irrtumswahr- } \\
\text { scheinlichkeit }\end{array}$ & Signifikanz & signifikanzniveau \\
\hline $0.25<\alpha$ & $\mathbf{s}<0.75$ & nicht signifikant \\
\hline $0.10<\alpha \leq 0.25$ & $0.75 \leq \mathbf{s}<0.90$ & tendenziell \\
\hline $0.05<\alpha \leq 0.10$ & $0.90 \leq \mathbf{s}<0.95$ & schwach signifikant \\
\hline $0.025<\alpha \leq 0.05$ & $0.95 \leq \mathbf{s}<0.975$ & signifikant \\
\hline$\alpha \leq 0.025$ & $0.975 \leq \mathbf{s}$ & stark signifikant \\
\hline
\end{tabular}

Die Stärke eines Zusammenhanges zweier Merkmale wird durch den Korrelationskoeffizienten gemessen. Die stärke des zusammenhanges ist in Tabelle 3.4 dargestellt, wobei $\mathrm{zu}$ berücksichtigen ist, daß nicht alle Korrelationsmaße Werte von 0 bis 1 annehmen, 41 sondern entsprechend dem jeweiligen Höchstwert umgerechnet werden müssen. 42

40 In Anlehnung an Selig, J.: EDV-Mangagement, Eine empirische Untersuchung der Entwicklung von Anwendungssystemen in deutschen Unternehmen, Berlin, Heidelberg, New York, Tokyo 1986.

41 So ist z.B. bei einer Vierfeldertafel die obergrenze für den Kontingenz-Koeffizienten $C=0,707$.

$42 \mathrm{Vgl}$. Siegel, S.: Nichtparametrische statistische Methoden, a.a.O., S. 192 f. 
Tabelle 3.4: Interpretation des Korrelationskoeffizien$\operatorname{ten}^{43}$

\begin{tabular}{|c|c|}
\hline Korrelationskoeffizient & Zusammenhang \\
\hline $0.0<r \leq 0.2$ & sehr niedriger Zusammenhang \\
\hline $0.2<r \leq 0.4$ & niedriger Zusammenhang \\
\hline $0.4<r \leq 0.7$ & mittlerer Zusammenhang \\
\hline $0.7<r \leq 1.0$ & hoher Zusammenhang \\
\hline
\end{tabular}

Die statistischen Kennzahlen werden im folgenden lediglich als ergänzende Information dargestellt. Zur Verdeutlichung der festgestellten zusammenhänge wird das erhobene Datenmaterial direkt vorgestellt.

43 In Anlehnung an Heller, K.; Rosemann, B.: Planung und Auswertung empirischer Untersuchungen, 2. Auflage, stuttgart 1981 , S. 122 . 


\subsection{Analyse der Ergebnisse}

\subsubsection{8truktur der antwortenden Unternehmen}

Die strukturellen Daten der Erhebung wurden zur Charakterisierung der Unternehmen und zur Zuordnung innerhalb des Explorationsschemas verwendet. Sie wurden speziell daraufhin untersucht, inwieweit sie als Determinanten für die Telekommunikatinsadoption signifikant sind. Mit Hilfe dieser strukturdaten werden die Verhaltensschemata der Unternehmen erstens auf signifikante Unterschiede und zweitens auf Korrelationen hin untersucht.

\subsubsection{Regionale Verteilung und Branchenstruktur}

\section{Regionale verteilung}

Die regionale Verteilung der Unternehmen basiert auf dem Indikatorensystem der Gemeinschaftsaufgabe 'Verbesserung der regionalen Wirtschaftsstruktur'. ${ }^{44}$ Als zu untersuchende Regionen wurden definiert:

- Großraum Frankfurt

- Großraum Kassel
- Großraum Gießen

- Großraum Fulda

Dabei nimmt Kassel den Raum Nordhessen, Gießen den Raum Mittelhessen, Fulda den Raum Osthessen und Frankfurt den Raum Südhessen ein.

In den vier Regionen wurden jeweils ca. 200 Unternehmen der ausgewählten Branchen befragt. Das Antwortverhalten läßt

44 Siehe Kapitel 3.1.2.1. 
ein starkes Nord-Süd-Gefälle erkennen, wobei Frankfurt mit $31 \%$ eindeutig an der spitze liegt, gefolgt von Gießen mit $11 \%$, Kassel mit $10 \%$ und Fulda mit $7 \%$. Die folgende Graphik stellt die prozentuale Verteilung der Antworten innerhalb der Grundgesamtheit dar, wobei Frankfurt eindeutig überproportional vertreten ist.

Abbildung 3.2: Prozentuale Verteilung der Antworten auf die einzelnen Regionen

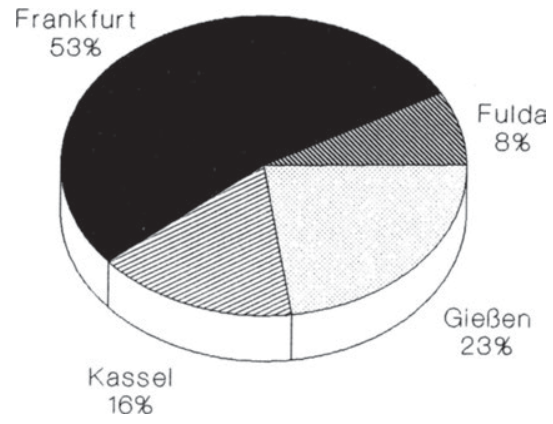

Unter Berücksichtigung der gesamten Ergebnisse kann festgestellt werden, daß in der Tendenz die Regionen überrepräsentiert sind, die einen Vorsprung in der Telekommunikations-Nutzung aufweisen.

\section{Branchenstruktur}

Die zu untersuchenden Branchen waren:

- Unternehmensberatungen

- Einzelhandel
- Software-Firmen/EDV-Vertrieb

- Maschinenbau 
Die vorgenommene Auswahl schloß eine Sektoren-Repräsentativität aus.

Abbildung 3.3 veranschaulicht, daß die realisierte struktur der stichprobe auf eine unterschiedliche Antwortbereitschaft der Befragten hindeutet, wobei die Unternehmensberatungen mit $37 \%$ die größte Antwortbereitschaft zeigen. Mit Abstand folgen der Maschinenbau mit 27\%, die Software-Firmen mit $21 \%$ und, als Gruppe mit der geringsten Antwortbereitschaft, der Einzelhandel.

Abbildung 3.3: Prozentuale Verteilung der Antworten auf die einzelnen Branchen

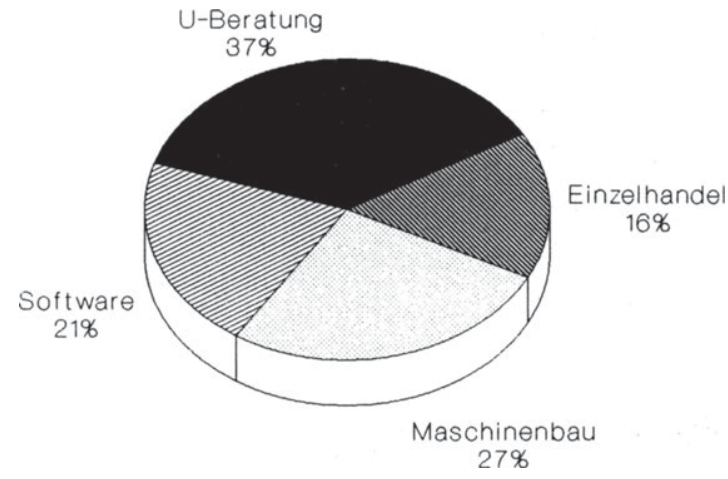

Auch hier kann, unter Berücksichtigung der gesamten Ergebnisse, von einer Überrepräsentativität der Unternehmen gesprochen werden, die einen Telekommunikations-Vorsprung aufweisen. 


\section{Rorrelationen}

Der detailierte Vergleich des Antwortverhaltens der verschiedenen Branchen je Region zeigt, 45 daß die Region Frankfurt, bedingt durch das hohe Antwortverhalten der Unternehmensberatungen, überrepräsentiert ist. In Fulda dominieren dagegen die Bereiche Maschinenbau und Einzelhandel. In Kassel und Gießen halten sich die Unternehmensberatungen und der Maschinenbau in etwa die Waage.

Abbildung 3.4: Antwortverhalten nach Region und Branche

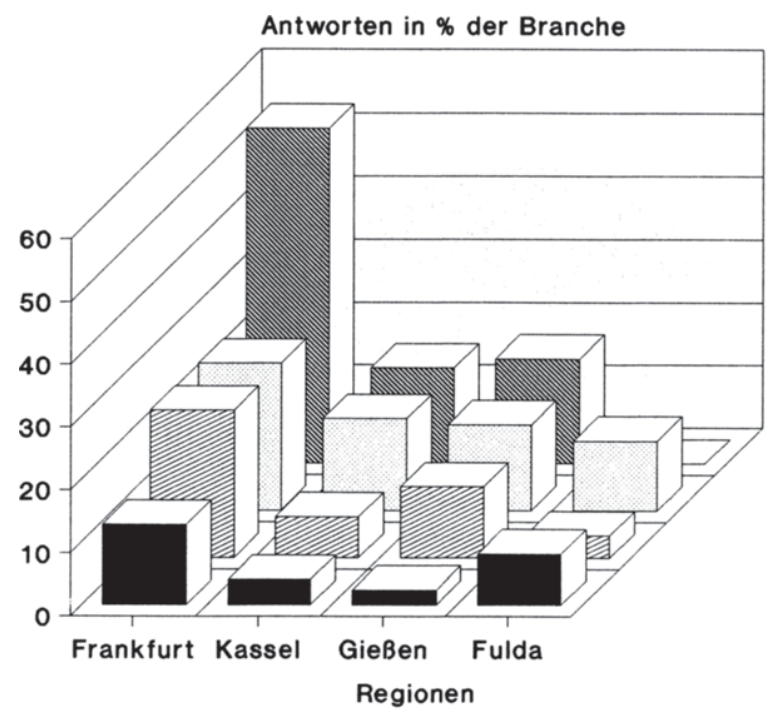

\begin{tabular}{|ll|}
\hline Einzelhandel & Software \\
$\square$ Masch.-Bau & $\mathbb{W}$ U-Beratung \\
\hline
\end{tabular}

Wie Abbildung 3.5 veranschaulicht, ist das Antwortverhalten des Einzelhandels, abgesehen von Fulda, unterproportional.

$45 \mathrm{Vgl}$. Abbildung 3.4. 
Die Antwortbereitschaft der verschiedenen Sektoren ist über die Regionen hin disparat. Dies deutet auf einen Zusammenhang zwischen dem regionalen Bewußtsein der Unternehmen und ihrer Antwortbereitschaft hin.

Abbildung 3.5: Gewichtung der Sektoren nach Regionen

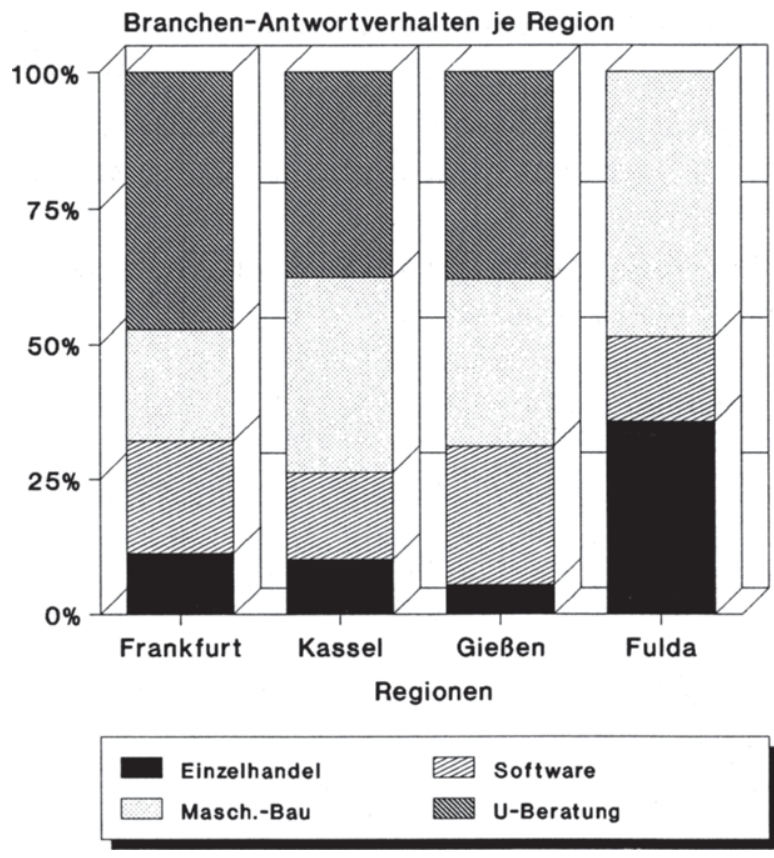

Überprüft man den Zusammenhang des Branchen-Antwortverhaltens nach Regionen mit Hilfe des Chi-Quadrat-Testes, so errechnet sich eine signifikanz von 0.98 , womit ein stark signifikanter Zusammenhang ${ }^{46}$ gekennzeichnet ist.

$46 \mathrm{Vgl}$. Tabelle 3.3 . 


\subsubsection{Beschäftigtenzahl und Unternehmensumsatz}

\section{Beschäftigenzahl}

Als Maßstab zur Einschätzung der Unternehmensgröße wurde die Zahl der beschäftigten Mitarbeiter gewählt.

Abbildung 3.6 zeigt den prozentualen Anteil der nach Grössenklassen unterteilten Unternehmen. Die Kleinunternehmen (bis 100 Beschäftigte ${ }^{47}$ ) überwiegen mit einem Anteil von $94 \%$ der befragten Unternehmen. Die verbleibenden $6 \%$ sind Unternehmen mittlerer Größe, mit 100-500 Beschäftigten.

Abbildung 3.6: Anzahl der Unternehmen nach Beschäftigtengrößenklassen

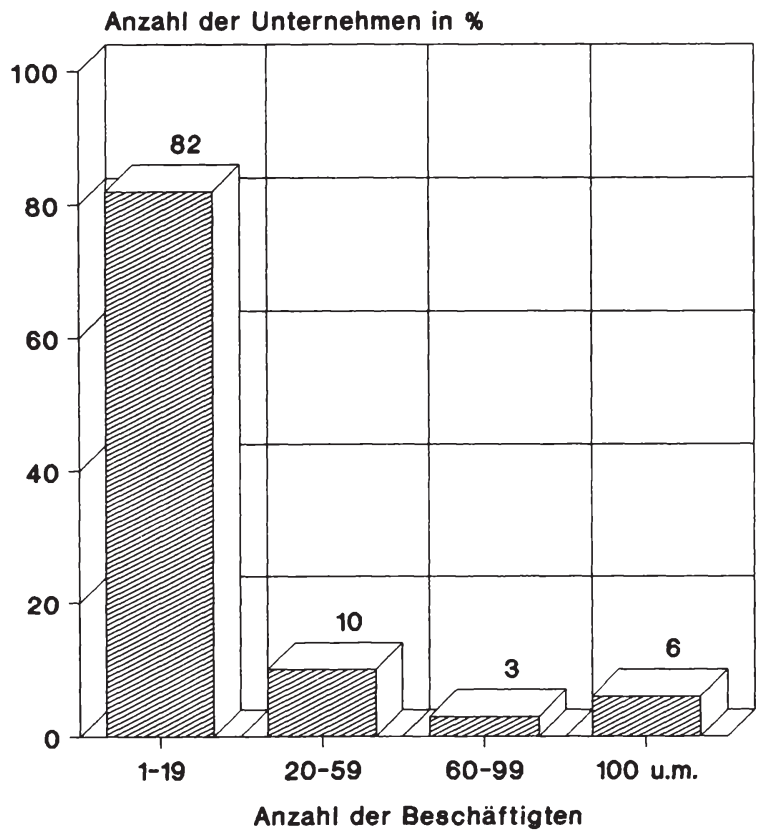

47 Vgl. Fölber, R.: Bedarfsuntersuchung für die Fernmeldenetze in Rheinland-Pfalz, in: Deutsche Telepost Consulting $\mathrm{GmbH}$ (Hrsg.): Aspekte der Telekommunikationsentwicklung im Land Rheinland-Pfalz, a.a.O., S. 20. 
Bei der Betrachtung der Größenklassen-Repräsentativität fällt die Dominanz der Kleinstunternehmen auf. Dieses Ergebnis entspricht weitgehend der realen Wirtschaftsstruktur in Hessen und der Forderung, die Erhebung auf klein- und mittelständige Unternehmen $z$ u beschränken. ${ }^{48}$

\section{Unternehmensumsatz}

Der Unternehmensumsatz wurde als zusätzlicher Maßstab zur Relativierung der Beschäftigtenzahl, als Maß für die Unternehmensgröße, gewählt. Dies ist besonders bei Unternehmensberatungen notwendig, die relativ wenig Mitarbeiter beschäftigen, jedoch durch ihre Qualifikationsstruktur einen höheren Umsatz realisieren können. Diese Charakterisierung wird durch Abbildung 3.7 verdeutlicht. 25\% der Unternehmen haben einen Umsatz von über fünf Millionen DM und 57\% von über einer Millionen DM. Unter 0.5 Millionen DM liegen immerhin noch $23 \%$ der Unternehmen, was zum großen Teil durch sogenannte 'Ein-Mann-Unternehmen' in der Unternehmensberatung bzw. Softwareentwicklung begründet ist.

Abbildung 3.7: Anzahl der Unternehmen nach Umsatzklassen in $\%$

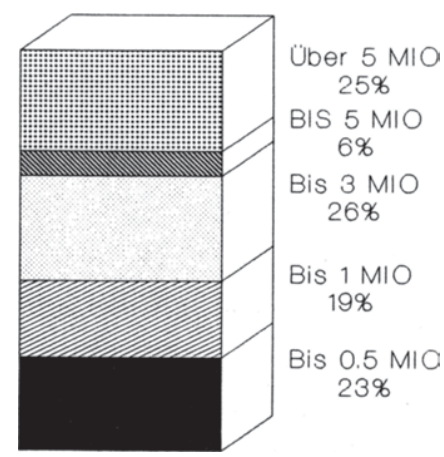

$48 \mathrm{Vgl}$. Winand, U. u.a.: Telekommunikation in Hessen, a.a.0., s. 11 . 


\subsubsection{EDV-Ausstattung und Forschungs- und Entwicklungs- aktivitäten}

\section{EDV-Ausstattung}

Zur Klassifizierung der Unternehmen nach ihrer EDV- und Innovationsorientierung als mögliche Determinanten für das Verhalten bezüglich neuer telekommunikativer Techniken, wurden die Erhebungsmerkmale EDV- Ausstattung und Forschungs- und Entwicklungsaktivitäten gewählt.

Eine EDV-Ausstattung in Form von Mikro- bzw. Personal Computern ist bei $77 \%$ der Unternehmen vorhanden, wobei $50 \%$ der Unternehmen mit EDV bereits mehr als drei Geräte besitzen. Abbildung 3.8 verdeutlicht diesen Zusammenhang.

Abbildung 3.8: PC-Ausstattung der Unternehmen

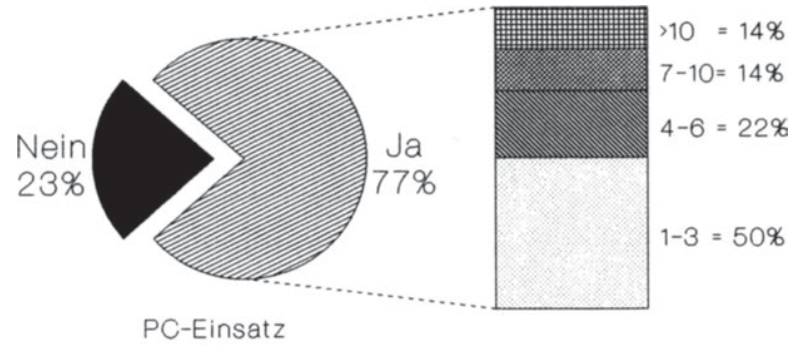

Anzahl

Signifikante Unterschiede bezüglich der EDV-Ausstattung zeigen beim Chi-Quadrat-Test die unterschiedlichen Branchen und Regionen. An der Spitze der EDV-Ausstattung liegen die Software-Firmen (100\%), gefolgt von den Unternehmensberatungen ( $80 \%$ ) und dem Maschinenbau (70\%). Beim Einzelhandel (40\%) ist die EDV-Ausstattung unterrepräsentiert. Das Signifikanzniveau der Branchenanalyse ist 'stark signifikant'. Die EDV-Ausstattung nach Regionen zeigt, daß Frankfurt (77\%), Kassel (79\%) und Gießen (89\%) auf ähnlichem Niveau 
liegen. Fulda (44\%) fällt dagegen erheblich ab. Das Signifikanzniveau bezüglich der Regionen ist 'signifikant' und der Korrelationskoeffizient weist auf einen 'mittleren zusammenhang' hin.

\section{Forschungs- und Entwicklungsaktivitäten}

Forschung und Entwicklung wird, wie in Abbildung 3.9 dargestellt, von $44 \%$ der befragten Unternehmen betrieben. Differenziert man die Forschungs- und Entwicklungsaktivitäten nach den untersuchten Branchen, so ergeben sich 'stark signifikante' Unterschiede. Führend sind die Software-Firmen mit einem Anteil von 75\%, gefolgt vom Maschinenbau mit 60\%, den Unternehmensberatungen mit $36 \%$ und dem Einzelhandel mit $11 \%$.

Abbildung 3.9: Forschung und Entwicklung in den Unternehmen

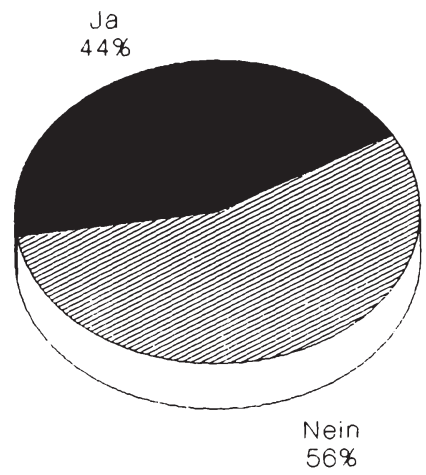

\section{Rorrelationen}

Erwartungsgemäß korrelieren EDV-Ausstattung und Forschungsund Entwicklungsaktivitäten, da der Bereich der SoftwareFirmen bezüglich der EDV-Ausstattung dominiert. Mit Hilfe des Chi-Quadrat-Tests läßt sich ein 'stark signifikanter' Zusammenhang nachweisen. $88 \%$ der Unternehmen, die Forschung und Entwicklung betreiben, nutzen eine EDV-Anlage, 
lediglich $12 \%$ forschen und entwickeln ohne EDV Unterstützung.

\subsubsection{Art und Gründungsjahr des Unternehmens}

\section{Art des Unternehmens}

Die Art des Unternehmens wurde durch zwei Variablen erfaßt. Zum einen durch die selbständigkeit, beziehungsweise organisatorische Einordnung des befragten Unternehmens und zum anderen durch die Anzahl der Unternehmensstandorte.

Zur Erhebung der ersten Variablen wurden die Unternehmen nach der Kategorie gefragt, der der Betrieb angehört. Als Antwortmöglichkeiten wurden vorgegeben:

- eigenständiges Unternehmen

- Zentrale
- Zweigstelle/Filiale

- Niederlassung/ Geschäftsstelle

Die Ergebnisse sind in Tabelle 3.5 zusammengefaßt. Sie dokumentiert, daß $85 \%$ der befragten Unternehmen eigenständig sind. Das strukturmerkmal 'Art des Unternehmens' wird aus diesem Grund im weiteren nicht berücksichtigt.

Tabelle 3.5: Art der Unternehmen

\begin{tabular}{|l|c|}
\hline Status & $\begin{array}{c}\text { Anzahl der Unternehmen } \\
\text { in } \% \text { der Grundgesamtheit }\end{array}$ \\
\hline Eigenständiges Unternehmen & $85 \%$ \\
\hline Zentrale & $7 \%$ \\
\hline Zweigstelle/Filiale & $4 \%$ \\
\hline Niederlassung/Geschäftsstelle & $4 \%$ \\
\hline
\end{tabular}


Die bei den Unternehmensbefragungen von Spehl/MesserigFunk 49 und Fritsch 50 nachgewiesene Korrelation zwischen der Unternehmensart und dem Adoptionsverhalten gegenüber den neuen Telekommunikationsdiensten, kann somit nicht belegt werden. Bei den Untersuchungen von Spehl/Messerig-Funk und Fritsch wurde weiterhin die Hypothese bestätigt, daß eigenständige Unternehmen neue Telekommunikationsdienste weniger nutzen als Betriebe, die zu Unternehmen mit mehreren Betrieben gehören. 51

\section{8tandortanzahl}

Als zweite Variable zur Beschreibung der Art des Unternehmens wurde die Anzahl der standorte erhoben. Von den befragten Unternehmen haben $29 \%$ mehrere Standorte. $69 \%$ haben zwischen einem und drei, $14 \%$ zwischen vier und zehn und $17 \%$ mehr als zehn standorte. Korrelationen zu diesem Merkmal ergaben sich nicht.

Abbildung 3.10: Anzahl der Unternehmen mit mehreren standorten

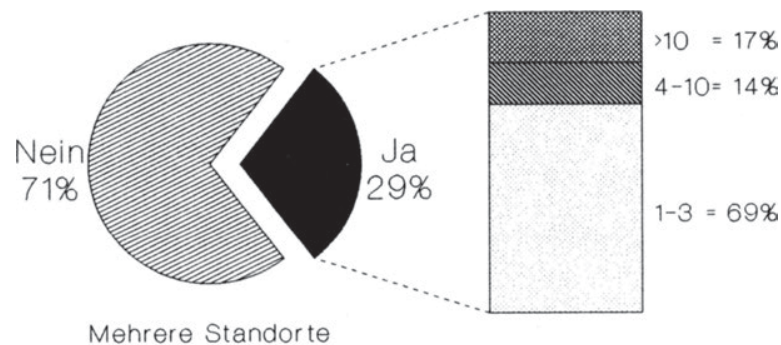

Anzahl

49 Vgl. Spehl, H.; Messerig-Funk, B.: Chancen und Probleme der wirtschaftlichen Nutzung der Telematik in einer ländlichen Region: Trier, a.a.0., S. $417 \mathrm{ff}$.

$50 \mathrm{Vgl}$. Fritsch, M.: Räumliche Unterschiede der Telematikadoption in Industriebetrieben der Bundesrepublik Deutschland, a.a.0., s. 301-335.

51 Vgl. Schnöring, T.: Wirkungen der Telekommunikationsentwicklung auf Anwender, a.a.0., S. $48 \mathrm{ff}$. 


\section{Grüdungsjahr der Unternehmen}

Bei den befragten Unternehmen handelt es sich, wie aus Abbildung 3.11 ersichtlich, zu 63\% um Betriebe, die bis 1983 gegründet wurden. Die restlichen $37 \%$ verteilen sich auf die Jahre 1984 bis 1988, wobei 1987 mit $15 \%$ besonders hervorsticht.

Abbildung 3.11: Gründungsjahr der Unternehmen

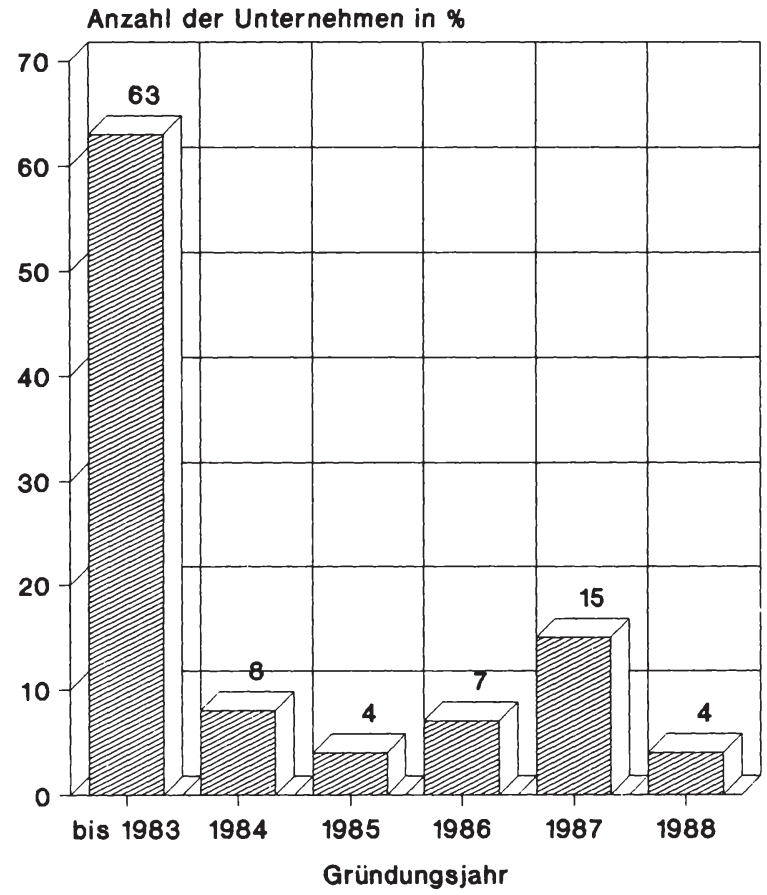

Um eine Korrelation der Unternehmen gegenüber dem Einflußfaktor Telekommunikation auf die standortentscheidung $z u$ überprüfen, wurden die Unternehmen in zwei Gruppen unterteilt. Die erste Gruppe besteht aus Unternehmen, die bis einschließlich 1985 gegründet wurden und die zweite Gruppe aus Unternehmen, deren Gründungsjahr im Zeitraum von 1986- 
1988 liegt. Berücksichtigt man die Vorlaufzeit für eine Neugründung, so kann davon ausgegangen werden, daß bei den Unternehmensgründungen vor 1986, aufgrund der mangelnden Informationen über ISDN, neue telekommunikative Techniken als standortfaktor keinen Einfluß hatten.

Abbildung 3.12: Gründungsjahr der Unternehmen in Klassen

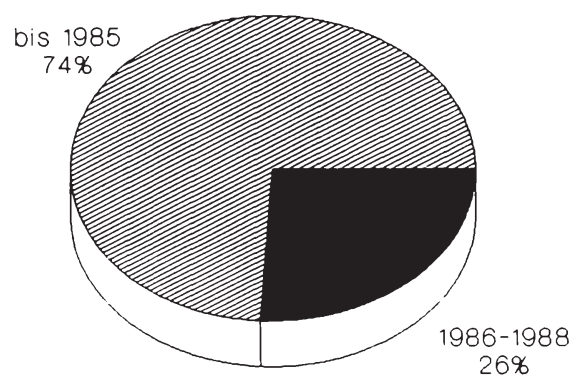

Die als Neugründungen definierten Unternehmen stellen mit $26 \%$ der Grundgesamtheit einen beachtlichen Teil der befragten Unternehmen dar. Ein signifikanter Zusammenhang ergibt sich zu den Merkmalen 'PC-Einsatz' und 'Branche'. Sämtliche neugegründeten Unternehmen setzen EDV in ihrem Unternehmen ein. Bei der Branchenzusammensetzung zeigt sich, daß 53\% der Neugründungen im Bereich der Sofware-Firmen liegen, dicht gefolgt von den Unternehmensberatungen mit 37\%. Beim traditionellen Maschinenbau lagen keine Neugründungen vor und der Einzelhandelsanteil liegt bei $10 \%$. 


\subsubsection{Einstellung der Unternehmen gegenüber der Telematik}

\subsubsection{Kenntnis- und Adoptionsstand}

Die in Tabelle 3.6 dargestellte Übersicht verdeutlicht den Kenntnisstand, den geplanten Einsatz und den Adoptionsstand der neuen telekommunikativen Techniken. Die jeweiligen Werte geben den Prozentwert der Grundgesamtheit, bezogen auf den Telekommunikationsdienst, an. Der Mittelwert bezieht sich auf eine Rangskala von ' $0=$ nicht bekannt' bis ' $3=$ heute genutzt'. Der Mittelwert in Abbildung 3.13 basiert auf der Grundgesamtheit aller befragten Unternehmen.

Um eventuelle zusammenhänge innerhalb der verschiedenen Kommunikationsgruppen aufzuzeigen, wurden die neuen Telekommunikationstechniken, entsprechend ihrer Zugehörigkeit, in vier Gruppen untergliedert. Die vier Gruppen sind

- die Sprachkommunikation,

- die Textkommunikation,

- die Datenkommunikation und

- die Bildkommunikation. 52

Tabelle 3.6 enthält nicht die Frage nach der Nutzung des reinen Fernsprechens, da dies für fast $100 \%$ der Befragten von großer Bedeutung ist (Siehe Abbildung 3.17) und dieser Dienst heute in fast allen Unternehmen vertreten ist.

Die in Tabelle 3.6 aufgeführten Dienste im Bereich der Sprachkommunikation, nehmen im Vergleich zum Fernsprechen eine untergeordnete Rolle ein. Ausgenommen hiervon ist

52 siehe Abbildung 1.4. 
Tabelle 3.6: Adoptionsverhalten gegenüber den Telekommunikationsdiensten

\begin{tabular}{|c|c|c|c|c|c|}
\hline $\begin{array}{l}\text { Telekommunika- } \\
\text { tionsdienste in } \\
\text { \& der Befragten }\end{array}$ & $\begin{array}{l}\text { Heute } \\
\text { genutzt }\end{array}$ & $\begin{array}{l}\text { Einsatz } \\
\text { geplant }\end{array}$ & $\begin{array}{l}\text { Bekannt, Ein- } \\
\text { satz nicht } \\
\text { geplant }\end{array}$ & $\begin{array}{c}\text { Nicht } \\
\text { bekannt }\end{array}$ & $\begin{array}{c}\text { Mittel- } \\
\text { wert }\end{array}$ \\
\hline Funkfernsprechen & 26 & 13 & 47 & 15 & 1.5 \\
\hline Eurosignal & 13 & 4 & 70 & 13 & 1.2 \\
\hline Cityruf & -- & 2 & 73 & 25 & 0.8 \\
\hline GEDAN & & 6 & 49 & 36 & 0.9 \\
\hline Service 130 & 2 & 1 & 47 & 50 & 0.6 \\
\hline Sprachbox & -- & -- & 34 & 66 & 0.3 \\
\hline Telex & 41 & 1 & 55 & 3 & 1.8 \\
\hline Teletex & 13 & 4 & 78 & 5 & 1.3 \\
\hline Btx & 14 & 12 & 68 & 5 & 1.4 \\
\hline Telebox & -- & 5 & 55 & 40 & 0.7 \\
\hline electronic mail & 10 & 12 & 48 & 32 & 1.0 \\
\hline DFÜ mit Telefon & 37 & 13 & 33 & 18 & 1.7 \\
\hline Datex - L & 9 & 1 & 51 & 40 & 0.8 \\
\hline Datex - P & 18 & 10 & 39 & 34 & 1.1 \\
\hline$H \in D$ & 4 & 1 & 23 & 72 & 0.4 \\
\hline Temex & -- & 1 & 23 & 75 & 0.3 \\
\hline Telefax & 73 & 17 & 8 & 3 & 2.6 \\
\hline Bildtelefon & -- & 3 & 85 & 12 & 0.9 \\
\hline Videokonferenz & -- & 2 & 85 & 13 & 0.9 \\
\hline
\end{tabular}

lediglich das Funkfernsprechen, das mit 26\% den größten Anteil, neben dem normalen Fernsprechen, einnimmt. Auch der geplante Einsatz im Bereich des Funkfernsprechens liegt mit $13 \%$ an der spitze im Sprachbereich. Die neueren Dienste sind zum einen mit bis zu $66 \%$ bei Sprachbox, $50 \%$ beim Ser- 
vice 130 und $36 \%$ bei der Anrufweiterschaltung unbekannt und zum anderen ohne größeres Interesse (= bekannt, Einsatz nicht geplant).

Im Bereich der Textkommunikation dominiert der Telexdienst $(41 \%=$ heute genutzt), der jedoch zukünftig mit nur geringem Zuwachs rechnen kann $(1 \%)$. Teletex, Btx und electronic mail 53 nehmen, vom Nutzerprozentsatz her, einen geringeren Teil ein, weisen jedoch beim geplanten Einsatz, mit bis zu 12\%, ein entsprechendes Wachstumspotential aus. Der relativ hohe Anteil des geplanten Btx-Einsatzes resultiert wahrscheinlich aus der Zusammensetzung der Stichprobe, die sich hauptsächlich im Bereich der Kleinunternehmen bewegt.

Bei der Datenkommunikation ist die Datenübertragung im Telefonnetz mit $37 \%$ die am häufigsten genutzte Kommunikationsform, die auch den größten Zuwachs mit $13 \% \mathrm{zu}$ erwarten hat. Bei den anderen Diensten überrascht der hohe Anteil im Bereich 'nicht bekannt'. Mit Einführung des ISDN wird besonders die Datenübertragung im Telefonnetz mit weiteren Zuwächsen rechnen können.

Die Bildübertragung enthält mit dem Telefax, das bereits von $73 \%$ der befragten Unternehmen genutzt wird, den neben dem Fernsprechen am häufigsten anzutreffenden Dienst, der mit $17 \%$ geplanten Einsatz den stärksten Nutzerzuwachs erwarten läßt. Die noch sehr jungen Dienste Bildtelefon und Videokonferenz sind bei den meisten Unternehmen bekannt, der Einsatz ist jedoch nur von 2-3\% der Unternehmen geplant.

53 Electronic mail wird in seiner Urform noch nicht als Telekommunikationsdienst von der Deutschen Bundespost angeboten. Electronic mail ist jedoch inhouse in größeren Unternehmen bereits realisiert und adaptiert. Weltweit z.B. für die Unternehmenskommunikation von IBM, mit dem von IBM entwickelten System 'Office Vision 2000'. 
Ausgehend von den Mittelwerten der jeweiligen Antworten, läßt sich aus Abbildung 3.13 die unterschiedliche Bedeutung der Dienste ablesen. Dominierend ist eindeutig der Telefaxdienst mit dem Wert von 2.6, mit Abstand gefolgt vom Telexdienst mit 1.8, der Datenübertragung im Telefonnetz mit 1.7, dem Funkfernsprechen mit 1.5 und dem Btx mit 1.4. Die restlichen Dienste schwanken $2 w i s c h e n ~ 0.7$ und 1.1 , ausgenommen dem Service 130, der Sprachbox, der HfD und dem Temexdienst, die bei 0.3 bis 0.4 Punkten liegen. Bei der Interpretation dieser Ergebnisse muß jedoch die Branchenzusammensetzung und das regional differenzierte Kommunikationsverhalten des samples berücksichtigt werden.

Abbildung 3.13: Mittelwerte des Adoptionsverhaltens

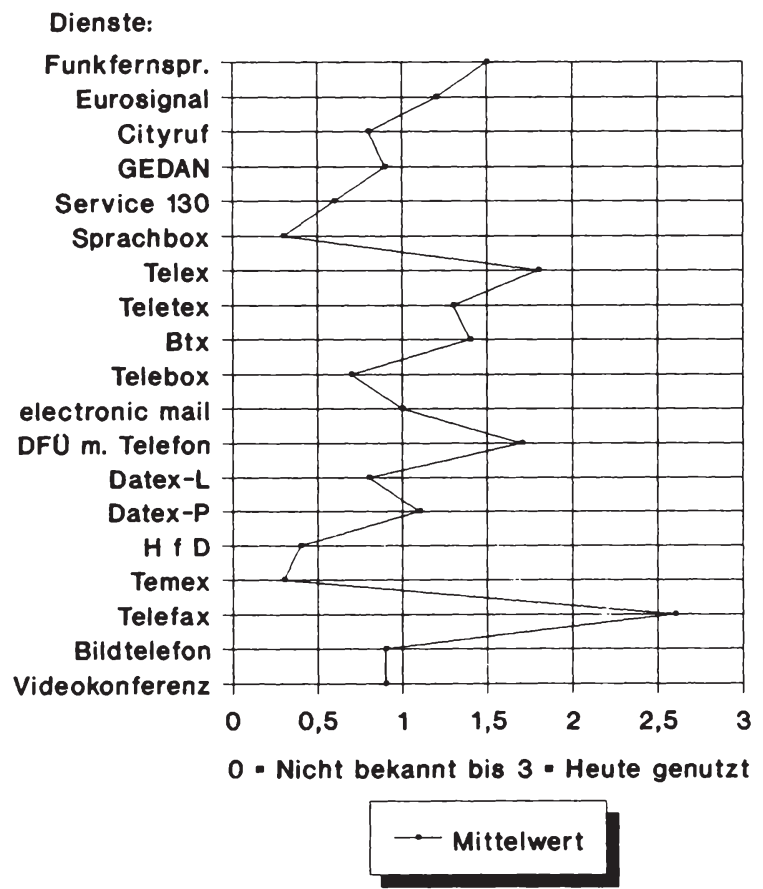


Das Kommunikationsverhalten nach Regionen, wie in Abbildung 3.14 dargestellt, läßt sich zum Teil durch die unterschiedliche Branchenstruktur erklären. Einen weiteren wesentlichen Erklärungsfaktor stellt das regional vorhandene Informationsgefälle dar, was in Abschnitt 3.2.2.3. näher erläutert wird, das jedoch nicht einheitlich für alle Dienste gleichermaßen zutrifft.

Abbildung 3.14: Mittelwerte des Adoptionsverhaltens nach Regionen

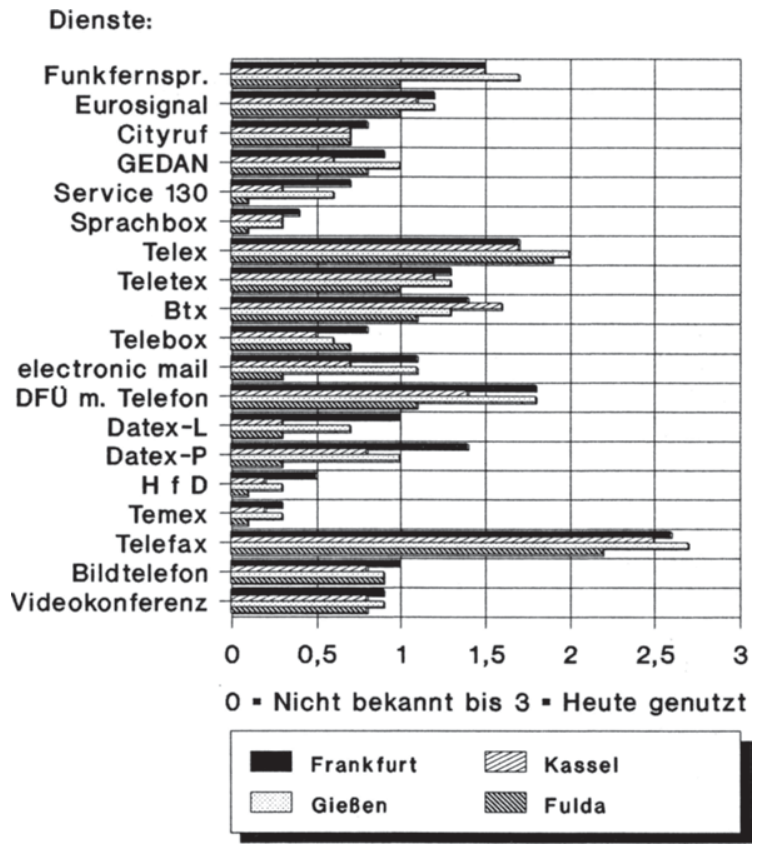

Regionale Disparitäten im Adoptionsverhalten liegen im Bereich der speziellen sprachkommunikationsdienste und der Datenkommunikation. Hier läßt sich ein starkes Nord-Süd-Gefälle feststellen. Ausgehend von Frankfurt, dicht gefolgt von Gießen, liegt Kassel teilweise bis über $50 \%$ unter dem 
Wert von Frankfurt, wobei Fulda den Abschluß bildet. Bei den dominierenden Kommunikationsdiensten Telefax, Telex, Funkfernsprechen und Btx, liegt Frankfurt nicht an der Spitze, sondern wird von Gießen knapp überragt. Der unerwartet hohe Anteil von Telex und Telefax im Bereich Fulda, resultiert aus dem hohen Branchenanteil der Maschinenbaufirmen in dieser Region.

\section{Rorrelationen}

Zur Überprüfung signifikanter Zusammenhänge wurde, aufgrund des ordinalen Skalenniveaus, die "Ein-Weg-Rangvarianzanalyse nach Kruskal und Wallis" 54 gewählt.

Die bereits o.a. regionalen Disparitäten erweisen sich als signifikant55, wobei Frankfurt und Gießen etwa auf einer Ebene liegen und Kassel, mit leichtem Abstand zu Fulda, folgt. Dies gilt in signifikantem Ausmaß für die Dienste Service 130, electronic mail, Datex-L und Datex-P.

Definiert man die Größe eines Unternehmens durch die Anzahl der Beschäftigen und den Umsatz, so bestehen signifikante bis stark signifikante Zusammenhänge zwischen der Größe eines Unternehmens und dem Adoptionsverhalten. Mit wachsender Anzahl der Beschäftigten verändert sich das Adoptionsverhalten der Unternehmen, wobei der erste sprung bei Betrieben mit 20-39 Mitarbeitern festzustellen ist. Bei Unternehmen mit mehr als 60 Mitarbeitern nehmen die Telekommunikationsdienste ca. den doppelten Rangwert, im Vergleich zur Gruppe bis 19 Mitarbeiter, ein. Signifikant ist dies bei den Diensten Eurosignal, Service 130, Sprachbox, Telex, electronic mail, Datex-L und Datex-P. Erweitert und bestätigt wird diese Diensteliste durch den signifikanten

$54 \mathrm{Vgl}$. auch Hartung, H.: Statistik: Lehr- und Handbuch der angewandten Statistik, 6. Auflage, München, Wien, Oldenbourg 1987, S. $613 \mathrm{ff}$.

55 Ausgenommen vom Service 130 , sind die restlichen Telekommunikationsdienste stark signifikant. 
Zusammenhang zwischen Adoptionsverhalten und Unternehmensumsatz. Mit steigendem Umsatz werden vermehrt Telekommunikationsdienste adoptiert. Ein signifikanter Zusammenhang existiert beim Service 130, Telex, Teletex, HfD, und Telefax. Der markante Schwellenpunkt beim Jahresumsatz liegt bei 3 Mio. DM, wobei der Zusammenhang nicht in dem starken Ausmaß vorliegt wie bei der Beschäftigtenzahl. Die Rangwerte steigen relativ gleichmäßig an und bleiben im spitzenbereich beim ca. 1,5-fachen der kleinsten Umsatzgruppe.

Einen weiterer signifikanter Einfluß auf das Adoptionsverhalten resultiert aus der Branchenzugehörigkeit der Unternehmen. Stark signifikante Zusammenhänge zeigen die Dienste Funkfernsprechen, Eurosignal, Cityruf, Telex, electronic mail, Datenübertragung im Telefonnetz, Datex-L, Datex-P und Telefax. Hier ist jedoch kein homogener Zusammenhang festzustellen. Funkfernsprechen z.B. wird am stärksten von den Unternehmensberatern genutzt. Bei den restlichen signifikanten Diensten führt die Software-Branche die Rangliste an, dicht gefolgt von den Unternehmensberatungen und, mit erheblichem Abstand, dem Maschinenbau und dem Einzelhandel.

Aufgrund des signifkanten Zusammenhangs von Branche und PCEinsatz ${ }^{56}$, liegt erwartungsgemäß auch ein signifikanter $\mathrm{Zu}$ sammenhang zwischen EDV-Ausstattung und Adoptionsverhalten vor. Das Fehlen von Mikro- bzw. Personal-Computereinsatz ist ein signifikantes Merkmal für eine geringere Adoption neuer telekommunikativer Techniken. Die signifikanten Dienste entsprechen denen der Branchenkorrelationen, mit Ausnahme des Eurosignals. Zusätzlich ist der Btx-Dienst bei den PC-Anwendern signifikant, was aus dem hohen Anteil der Softwarebranche resultiert, die einen großen Teil der BtxAnwender umfaßt. Die Signifikanzen im Bereich Forschungsund Entwicklungsaktivitäten entsprechen, aufgrund der vorhandenen Korrelation, den Signifikanzen im Bereich PC-Ein-

56 Siehe Kapitel 3.2.1.3. 
satz. Unternehmen mit Forschungs- und Entwicklungsaufgaben akzeptieren und verwenden neue Telekommunikationstechniken deutlich häufiger als andere Betriebe.

Die neugegründeten Unternehmen weisen Signifikanzen beim Btx, electronic mail, Datenübertragung im Telefonnetz und beim Datex-P auf. Die Ergebnisse weisen nach, daß neugegründete Unternehmen neue Telekommunikationstechniken öfter adoptieren als bereits länger existierende Betriebe. Dies resultiert hauptsächlich aus der zusammensetzung der neugegründeten Unternehmen, die zum Großteil aus Unternehmensberatungen und Software-Firmen bestehen.

\subsubsection{Bedeutung der Dienste für den eigenen Betrieb}

Mit der Frage 14 wurde die Bedeutung der Dienste Telefon, Telex, Teletex, Btx, Telefax und Datenübertragung für den Betrieb abgefragt. Die Antwortmöglichkeiten lagen zwischen 'keine Bedeutung' (Rangwert=0) und 'von großer Bedeutung' (Rangwert=3). Abbildung 3.15 zeigt deutlich die unterschiedlichen stellenwerte der Dienste zueinander auf, wobei das Telefon von nahezu allen Betrieben als von großer Bedeutung eingestuft wird und die Rangfolge eindeutig anführt. Der Dienst Telefax nimmt mit 2.6 Punkten einen unerwartet hohen zweiten Rang ein, gefolgt von der Datenübertragung mit 1.9 punkten, Telex mit 1.0 punkten und als Abschluß Btx und Teletex mit 0.8 bzw. 0.7 punkten. 
Abbildung 3.15: Bedeutung der Telekommunikationsdienste

Dienste:

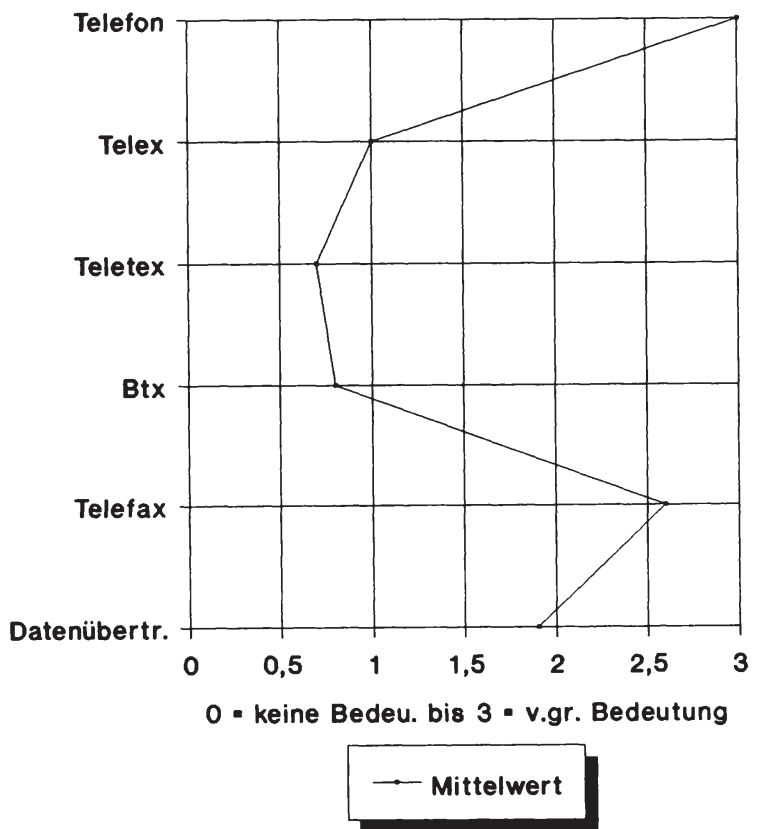

\section{Rorrelationen}

wie aus Abbildung 3.16 ersichtlich, sind nur geringe regionale Disparitäten festzustellen. Die Prüfung auf Signifikanz mit dem H-Test bestätigt dies. Lediglich im Bereich der Datenübertragung liegt Fulda bei der Beurteilung signifikant höher als die drei anderen Regionen. Die auffallend hohe Einschätzung des Telexdienstes in der Region Fulda erweist sich als nicht signifikant gegenüber den anderen Regionen. 
Abbildung 3.16: Bedeutung der Dienste nach Regionen

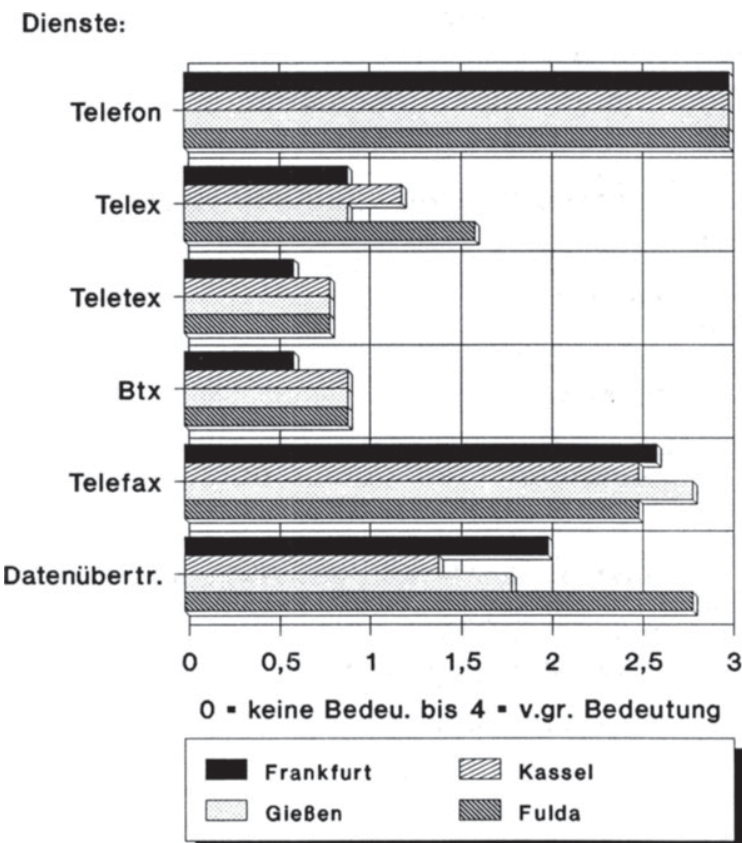

Weitere signifikante Unterschiede zeigen sich im Bereich der Unternehmensgröße beim Telex und Telefax, was dem Ergebnis beim Adoptionsverhalten entspricht.

Die Branchen verhalten sich bei der Bewertung der Bedeutung, abgesehen von der Kontrollgruppe Einzelhandel, insoweit homogen, als daß die Bedeutung der Dienste grundsätzlich höher eingeschätzt wird. Unternehmensberatungen, Softwarefirmen und der Maschinenbau schätzen die Bedeutung der Dienste Telefax, Btx und Teletex in etwa gleich ein. Beim Telex dominiert eindeutig der Maschinenbau und bei der Datenübertragung die Software-Firmen. Demgegenüber schätzt 
der Einzelhandel die Bedeutung der Dienste bis zu 50\% niedriger ein.

Abbildung 3.17: Bedeutung der Dienste nach Branchen

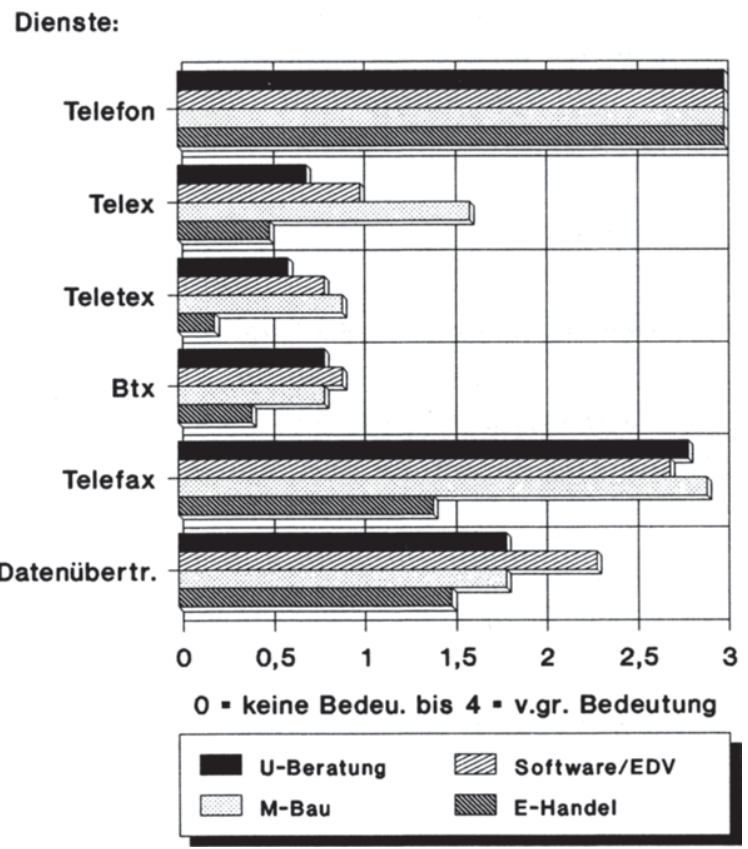

EDV-Ausstattung und Forschungs- und Entwicklungsaktivitäten verhalten sich signifikant bei Telex, Telefax und den Datenübertragungsdiensten.

Die einzigen Unternehmen, die signifikant dem Btx eine größere Bedeutung zusprechen, sind die neugegrüdeten unternehmen. Neben Btx sind für diese Gruppe Teletex und Datenübertragung von großer Bedeutung, die sogar etwashöher als Btx bewertet wurden. 


\subsubsection{Adoptionshemmnisse}

Bei der Beantwortung der Frage nach den Adoptionshemmnissen wurden verschiedene Antwortmöglichkeiten vorgegeben. Mehrfachnennungen waren möglich. Abbildung 3.18 zeigt die Rejektionsgründe unter regionalem Aspekt.

Abbidlung 3.18: Adoptionshemmisse nach Regionen

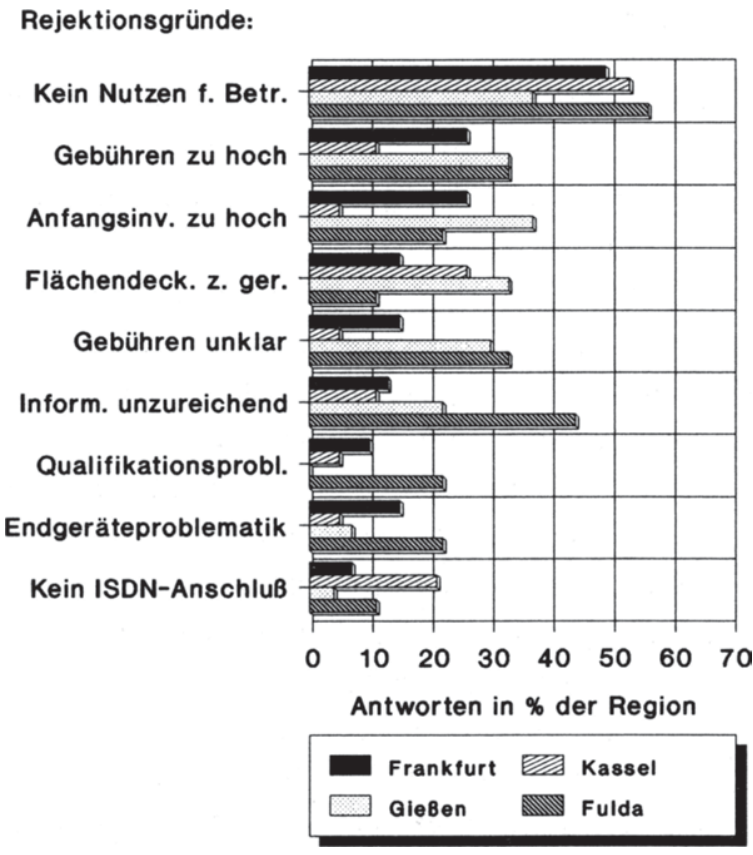

Das mit durchschnittlich 47\% am häufigsten angegebene Adoptionshemmnis war 'Kein Nutzen für den Betrieb', wobei in Gießen mit $37 \%$ dieses Adoptionshemmnis, im Vergleich $z u$ den anderen Regionen, am schwächsten ausgeprägt ist. Am zweithäufigsten wurde der Grund 'Gebühren $z u$ hoch' mit 26\%, gefolgt von 'Anfangsinvestitionen/Einführungskosten $z \mathrm{u}$ hoch', mit $25 \%$ angegeben. Beide Gründe sind für die Region Kassel, mit jeweils $11 \%$ bei den Gebühren und $5 \%$ bei den Anfangsin- 
vestitionen, von untergeordneter Rolle. Die 'geringe Flächendeckung' wurde von $21 \%$ genannt, wobei dieser Grund in Kassel und Gießen erheblich größere Bedeutung hat. Die 'unklare Gebührenstruktur' wurde mit $18 \%$ leicht über den 'unzureichenden Informationen' mit $17 \%$ genannt. Das Ende der Rangskala wird von der 'Qualifikationsproblematik', der 'Endgeräteproblematik' und dem Punkt 'keine Bereitstellung eines Anschlusses in absehbarer Zeit', mit jeweils unter $10 \%$, gebildet. Spehl/Messerig-Funk, 57 die Gesellschaft für Information und Datenverarbeitung 58 und die Detecon 59 , kommen in ihren Untersuchungen zu parallelen Ergebnissen.

Die auftretenden regionalen Disparitäten, die besonders für Fulda und Kassel gelten, sind in Abbildung 3.19 verdeutlicht. Die Abbildung zeigt die jeweilige regionale Gewichtung der Adoptionshemmnisse im Verhältnis zu den anderen Regionen. Offensichtlich liegt im Bereich Fulda ein erhebliches Informationsdefizit gegenüber den Informationsleistungen der Deutschen Bundespost, den Endgeräteherstellern und den Ausbildungsorganen vor. Kassel dominiert im Punkt 'Keine Bereitstellung eines Anschlusses in absehbarer Zeit' und ist bei den Gebühren und Anfangsinvestitionen unterproportional vertreten. Gießen dominiert im Bereich der 'mangelnden Flächendeckung', den zu hohen Gebühren und den Anfangsinvestitionen. Unterrepräsentiert ist Gießen im Bereich 'Qualifikationsproblematik', 'ISDN-Anschlußzeitpunkt' und bei der 'Endgeräteproblematik'. Frankfurt als Agglomerationsschwerpunkt verhält sich bei den Punkten 'Kein Nutzen für den Betrieb', 'Gebühren zu hoch' und 'Anfangsinvestitionen zu hoch' in etwa paritätisch, dokumentiert aber

57 Vgl. Spehl, H.; Messerig-Funk, B.: Chancen und Probleme der wirtschaftlichen Nutzung der Telematik in einer ländlichen Region: Trier, a.a.o., S. $417 \mathrm{ff}$.

$58 \mathrm{Vgl}$. Winand, U. u.a.: Telekommunikation in Hessen, a.a.0., S. $86 \mathrm{f}$.

59 Vgl. Fölber, R.: Bedarfsuntersuchungen für die Fernmeldenetze in Rheiland-Pfalz, a.a.0., S. $72 \mathrm{ff}$. 
seinen Ballungszentrumsvorteil bei den anderen Rejektionsgründen, wo es abgesehen, vom Punkt der Endgeräteproblematik, unterproportional vertreten ist.

\section{Abbildung 3.19: Regionale Gewichtung der Adoptionshemm- nisse}

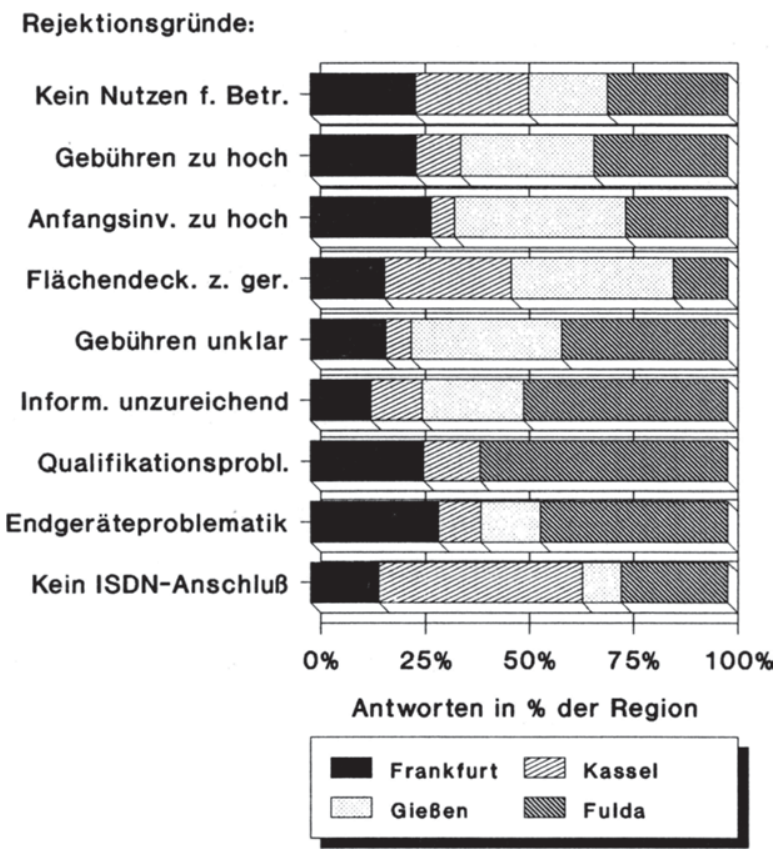




\subsubsection{EndgerätepreiseinfluB auf die Adoption}

Unter dem Aspekt des Minitels, das den Fernsprechteilnehmern in Frankreich kostenlos zur Verfügung gestellt wird und bis Ende 1989 mit ca. fünf Millionen Geräten auf dem französischen Markt vertreten ist, 60 wurde mit Frage 12 nach dem Endgerätepreis gefragt, bei dem die Dienste 61 adoptiert würden. Die Antwortmöglichkeiten der Dienste Telex, Teletex, Telefax und Btx reichten von einer skala von 0,- DM bis 5000,- DM, wobei zu jedem Dienst ein entsprechender Endgerätepreis angegeben werden sollte.

Der zusätzlich abgefragte Preis des Komfort-Telefons ergab einen Mittelwert von 80,- DM. Diesbezüglich lagen keine regionalen Disparitäten vor, so daß auf die nähere Darstellung verzichtet wird.

Ausgehend von Abbildung 3.20, welche die Mittelwerte der Antworten darstellt, steht Telefax mit 2.600,- DM an höchster stelle. Dies dokumentiert die Bedeutung, die diesem Dienst beigemessen wird. Die restlichen drei Dienste liegen in etwa auf einem Niveau, wobei Teletex mit 800,- DM leicht führt, gefolgt von Telex und Btx mit je 750,- DM.

$60 \mathrm{Vgl}$. o.V.: Der Minitel erobert in Frankreich die privaten Haushalte - Der elektronische Auftraggeber gehört schon zum täglichen Leben/Das Gerät ist gratis, berechnet wird die Benutzung, in: Frankfurter Allgemeine Zeitung Nr. 42 vom 19.02.1990, S.14.

61 Die Adoption der Dienste setzt per se die Anschaffung eines Endgerätes voraus, was in Form von Kauf, Mietkauf, Leasing $0 . \ddot{a}$. geschehen kann. 
Abbildung 3.20: Mittelwerte der potentiellen Endgeräteanschaffungspreise

Dienste:

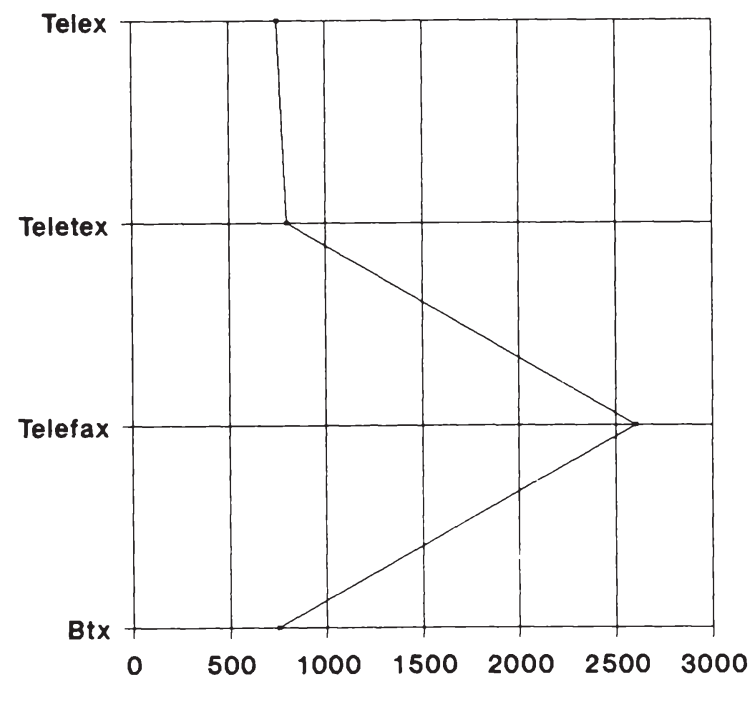

Antwormöglichkeit: DM O bis DM 5000

\section{- Mittelwert in DM}

\section{Rorrelationen}

Unter Berücksichtigung des regionalen Aspekts ergeben sich signifikante Unterschiede bezüglich des potentiellen Anschaffungspreises. Frankfurt als Ballungszentrum und somit am besten informiert über das momentane Peis-Leistungsverhältnis im Bereich der Telekommunikation, ist bei allen Diensten durch den niedrigsten Endgerätepreis vertreten. Signifikant ist dieses Verhalten für die Dienste Telex, Teletex und Telefax, wo es bis zu einer Differenz von 1000,DM im Verhälntis zu Gießen steht. Wie in Abbildung 3.21 dargestellt, dominiert Gießen in den Bereichen Telex, Teletex und Telefax, gefolgt von Kassel, daß leicht zurück liegt. 
Abbildung 3.21: Mittelwerte der potentiellen Endgeräteanschaffungspreise nach Regionen

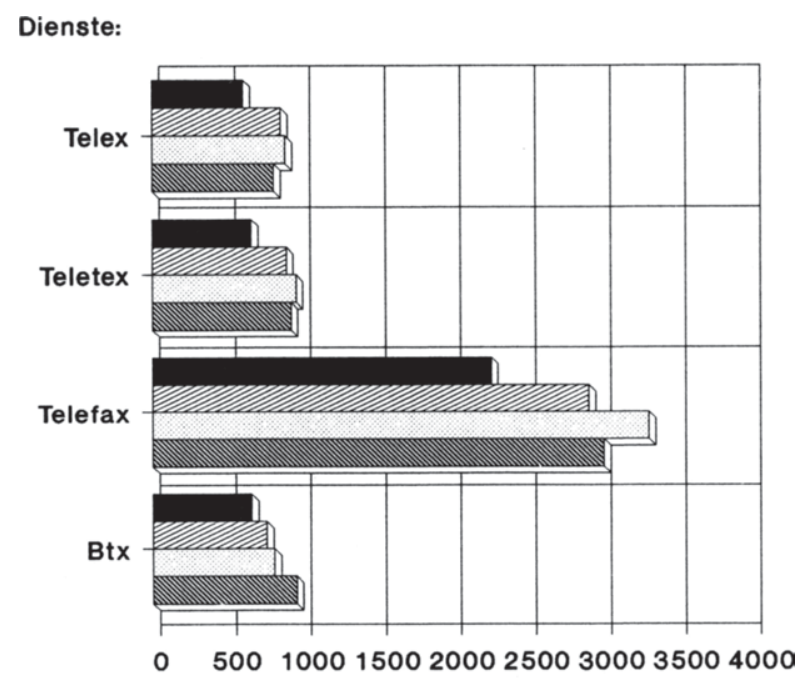

Antwortmöglichkeit: DM 0 bis DM 5000

\begin{tabular}{|ll|}
\hline Frankfurt & Kassel \\
$\square$ GieBen & $\mathbb{W}$ Fulda \\
\hline
\end{tabular}

Unerwartet hoch ist die Zahlungsbereitschaft in Fulda, die etwas über dem Großraum Kassel liegt und im Bereich Btx die spitzenposition einnimmt. 


\subsection{3. standorteinschätzung und standortverhalten}

Der Bereich Standorteinschätzung und standortverhalten wurde mit den Fragen '13. Wie bewerten sie Ihren momentanen Standort?', '16. Wie sind sie auf den jetzigen standort gekommen?' und '17. Welchen Einfluß hatten die im folgenden aufgeführten Faktoren auf die Auswahl des jetzigen standorts' abgefragt. Um eine Rangeinstufung bezüglich des ISDNAnbindungszeitpunktes und der Verfügbarkeit neuer telekommunikativer Techniken realisieren $\mathrm{zu}$ können, wurden neben diesen beiden Faktoren 21 traditionelle standortfaktoren ergänzend untersucht.

\subsubsection{Einschätzung des standortes}

Abbildung 3.22 dokumentiert die Einschätzungen bezüglich der aufgeführten standortfaktoren anhand der Mittelwerte aller Antworten. Mittels einer Rangskala von ' 0 =mangelhaft' bis ' $4=$ sehr gut' konnte pro standortfaktor je eine Wertung vergeben werden.

Den höchsten Wert erzielte der Faktor 'Anbindung an Straßen, Autobahnen' mit 3,2 Punkten. Die auf dem zweiten Rang liegende 'Nähe zu medizinischen Einrichtungen' liegt mit 2.9 Punkten leicht dahinter, gefolgt von den Faktoren 'Nähe zum Absatzmarkt' mit 2,8, 'Nähe zum Beschaffungsmarkt' mit 2,6 und 'Nähe zu Behörden', 'Anbindung an Bundesbahn' und 'Nähe zu Kultur- und Freizeiteinrichtungen' die mit jeweils 2,5 Punkten noch im oberen Bereich der Bewertung liegen. Die 'Nähe zur Konkurrenz', die 'Telekommunikations-Verfügbarkeit' und das 'Image/Umfeld' bilden mit je 2,4 Punkten, gefolgt von dem 'Reservoir an Hilfskräften' mit 2,3 und der 'Nähe zu Froschungseinrichtungen' und den 'Wohnstandortwünschenden von Angestellten' mit 2,2 Punkten, 
die letzten Werte im Bereich 'besser als befriedigend und gut'. Die Schlußlichter in der Bewertung liegen eindeutig bei den 'finanziellen Förderungen durch Kommunen', mit dem schlechtesten Wert von 0,5 und der 'finanziellen Förderung durch Bund und Land' mit dem wert 0.7.

Somit steht der Faktor 'zeitpunkt der I8DN-Anbindung' mit 2,0 punkten auf dem 9. Rang und die 'Telekommunikationsverfügbarkeit' mit 2,4 Punkten bereits auf Rang 6 .

\section{Abbildung 3.22: Momentane Standortbewertung}

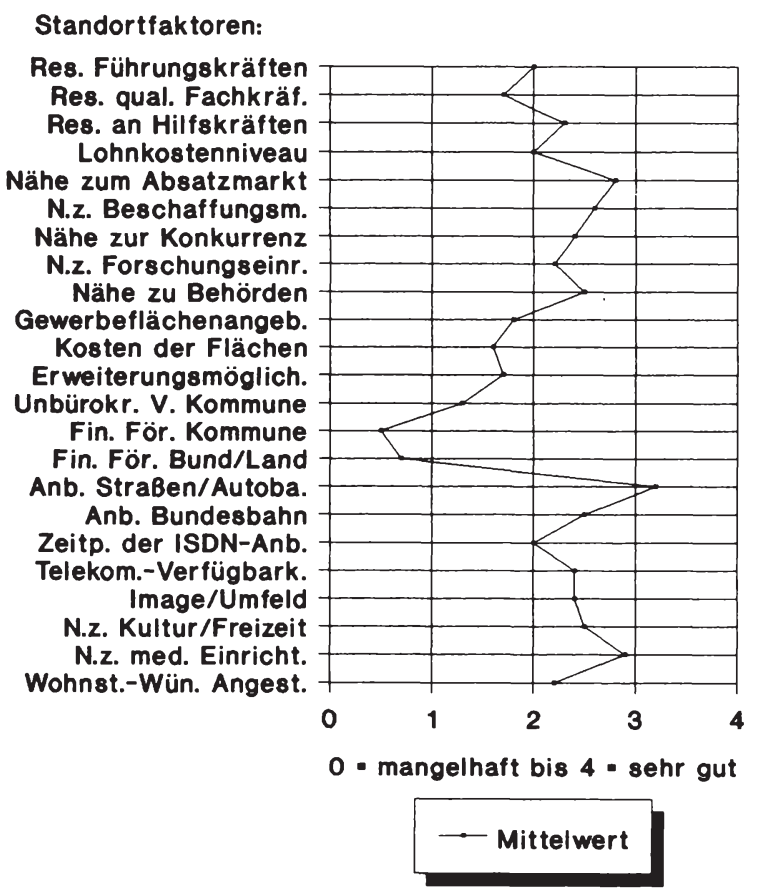

Dieses gute Ergebnis der beiden Telekommunikations-standorteinschätzungen relativiert sich unter dem Aspekt, daß beim 'Zeitpunkt der Anbindung an das ISDN-Netz' $53 \%$ und bei 
der 'Telekommunikations-Verfügbarkeit' $31 \%$ der Befragten angaben, diese Frage 'nicht beantworten zu können'.

\section{Abbildung 3.23: Momentane Standortbewertung des ISDN- Anbindungszeitpunktes}

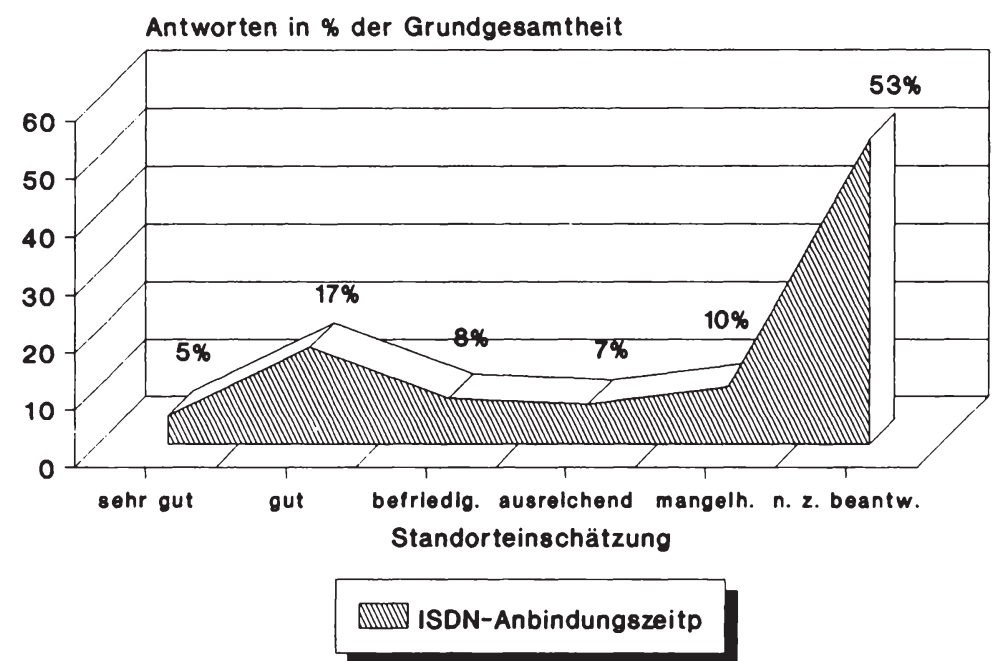

Unter Einbeziehung in eine alternative Rangskala, mit der Antwort 'nicht $z u$ beantworten' als niedrigste Rangstufe, ergibt sich für den ISDN-Anbindungszeitpunkt die drittschlechteste Bewertung mit 1,4 und für die Telekommunikations-Verfügbarkeit mit 2,4 der sechstschlechteste Wert, ausgehend von einer skala von Null bis Fünf.

\section{Rorrelationen}

Bei der standorteinschätzung besteht ein signifikanter zusammenhang hinsichtlich der unterschiedlichen Regionen. Frankfurt liegt hier als Agglomeration eindeutig an der Spitze der Bewertung. Das altindustrialisierte Kassel und die Region Fulda liegen in der Einschätzung mit Abstand hinter dem, im Vergleich zu Frankfurt, hoch eingschätzten Gießen. 
Der Standortfaktor 'Zeitpunkt der ISDN-Anbindung' ist der am stärksten disparat eingeschätzte Faktor. Er wird in Frankfurt und Gießen am höchsten eingeschätzt. Kassel und Fulda bewerten diesen Punkt um über $50 \%$ niedriger.

\section{Abbildung 3.24: Standorteinschätzung ausgewählter Faktoren nach Regionen}

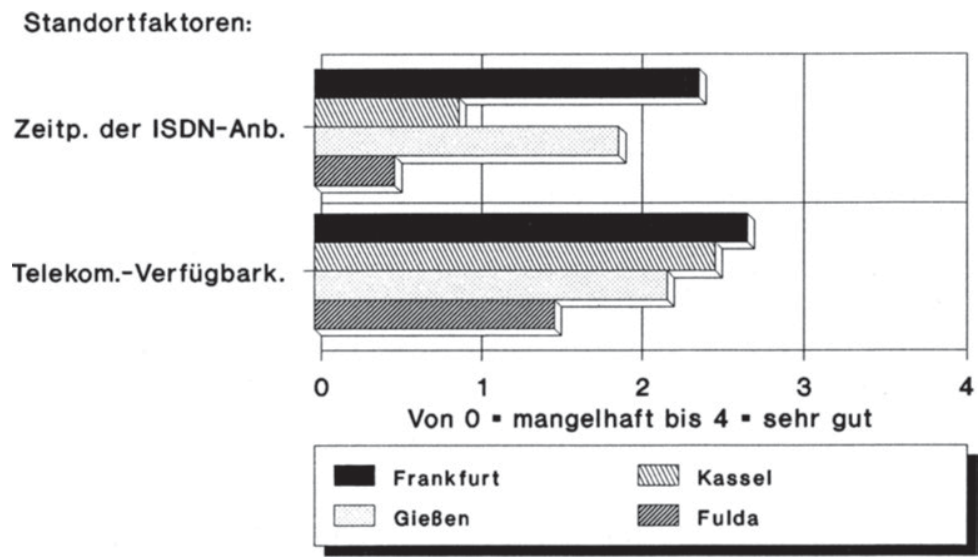

Der Standortfaktor 'Telekommunikations-Verfügbarkeit' wurde dagegen lediglich von Fulda signifikant schlechter eingeschätzt. Die höchste Einschätzung wurde in Frankfurt vorgenommen, gefolgt von Kassel und Gießen.

\subsubsection{Einfluß der standortfaktoren auf die standortwahl}

Die Frage nach dem Einfluß der standortfaktoren auf die Standortwahl wurde von bereits länger existierenden Unternehmen kaum beantwortet, da Informationen bezüglich der standortwahl nicht mehr vorlagen. Einige Unternehmen gaben 
an, daß die Standortwahl allein durch den Wohnsitz des Firmengründers determiniert war.

Abbildung 3.25: Einfluß der Standortfaktoren auf die Standortwahl

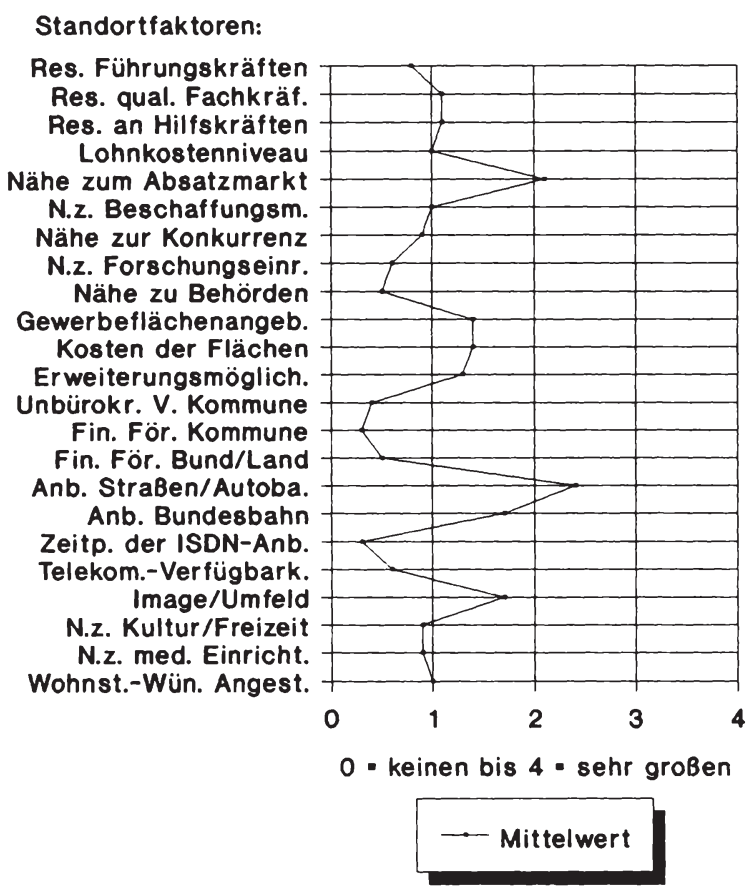

Wichtigster Einflußfaktor ist eindeutig die 'Anbindung an Straßen und Autobahnen', gefolgt von der 'Nähe zum Absatzmarkt', der 'Anbindung an die Bundesbahn' und dem 'Image und Umfeld'. Diese ersten vier Rangplätze liegen mit Abstand vor den restlichen Faktoren und dokumentieren ihre Bedeutung in Relation $z u$ den anderen Faktoren. Das 'Mittelfeld' der Faktoren bewegt sich zwischen 0,8 und 1,4 Punkten. Hier dominieren die Bereiche Arbeitskräfte- und 
Gewerbeflächenangebot, soziale Infrastruktur sowie die Nähe zum Beschaffungsmarkt und zur Konkurrenz. Die Faktoren mit dem geringsten Einfluß liegen im Bereich Nähe zu Behörden und Forschungseinrichtungen, finanzielle Förderungen und Telekommunikation. Den letzten Rangplatz nimmt der Faktor 'Zeitpunkt der ISDN-Anbindung' mit 0,3 Punkten ein. Der Faktor 'Telekommunikations-Verfügbarkeit' liegt mit 0,6 Punkten leicht darüber.

Abbildung 3.26 dokumentiert das Antwortverhalten bezüglich des Faktors 'ISDN-Anbindungszeitpunkt'. Lediglich $1 \%$ der Unternehmen gaben an, daß der ISDN-Anbindungszeitpunkt einen sehr großen Einfluß auf die standortwahl hatte. 62\% gaben an, daß der ISDN-Anbindungszeitpunkt keinen Einfluß hat.

Abbildung 3.26: Einfluß des ISDN-Anbindungszeitpunktes auf die standortwahl

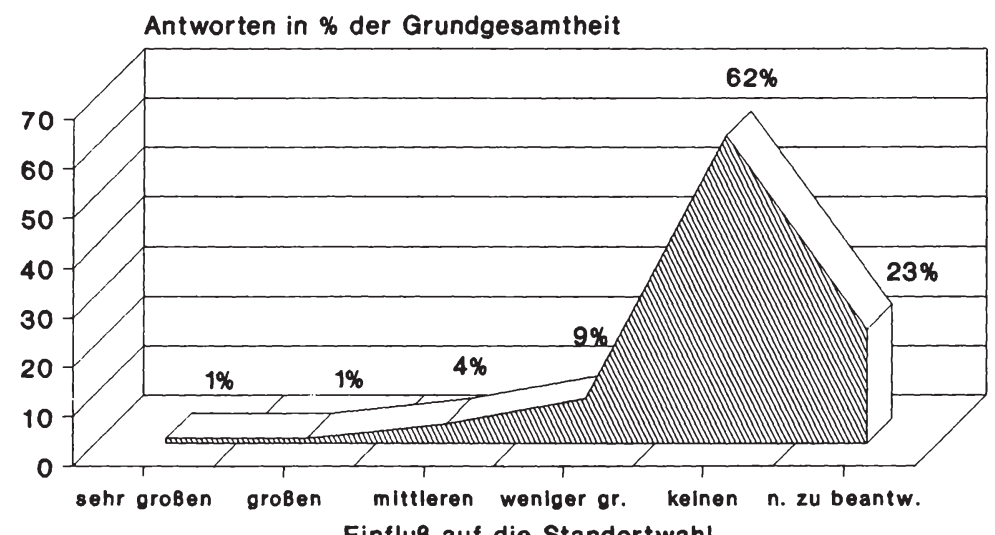

EinfluB auf die Standortwahl

Allell Zeitp. der ISDN-Anb. 
Im Bereich der Telekommunikations-Verfügbarkeit liegt der Einfluß bei einem Mittelwert von 0.6 Punkten um 0.3 Punkte höher und somit doppelt so hoch wie beim ISDN-Anbindungszeitpunkt. $12 \%$ der Unternehmen gaben an, daß ein 'mittlerer Einfluß' vorhanden war und $2 \%$ der Unternehmen gaben an, daß die Telekommunikationsverfügbarkeit einen 'sehr großen Einfluß' hatte. Abbildung 3.27 zeigt, daB bei $56 \%$ der Unternehmen die Telekommunikations-Verfügbarkeit ohne Einfluß auf die standortwahl blieb.

\section{Abbildung 3.27: Einfluß der Telekommunikations-Verfügbar- keit auf die standortwahl}

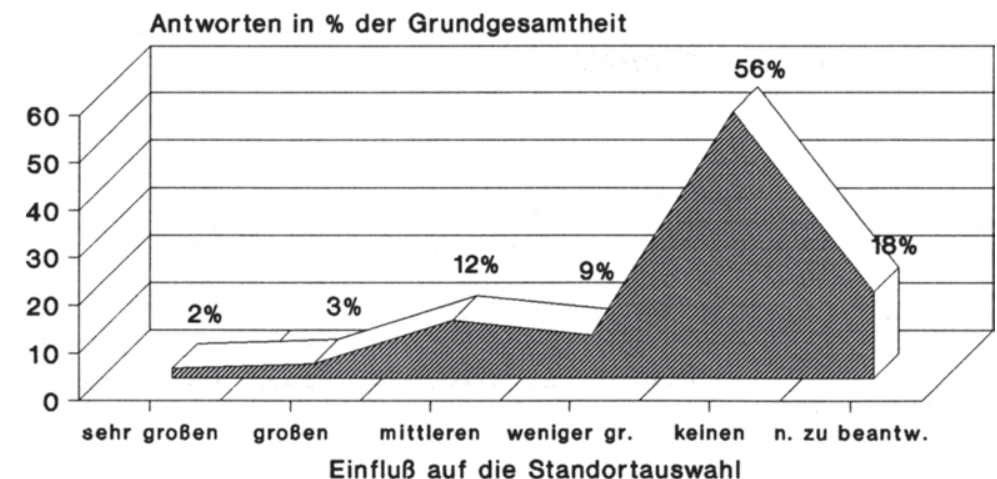

Einfluß auf die Standortauswahl

NTK-Verfügbarkeit

\section{Rorrelationen}

Aufgrund der insgesamt niedrigen Bewertung, hinsichtlich des ISDN-Anbindungszeitpunktes, herrscht in den vier Regionen statistisch gesehen lediglich eine schwache Tendenz vor, wobei Frankfurt und Gießen, im Verhältnis zu Kassel und Fulda, diesen standortfaktor erheblich höher bewerten. 
Bei der Telekommunikations-Verfügbarkeit liegt ein stärkerer signifikanter Zusammenhang in Form einer Tendenz bzw. schwachen Signifikanz vor. Wie aus Abbildung $3.28 \mathrm{zu}$ entnehmen ist, dominiert der Bereich Frankfurt und Gießen, wogegen Kassel und Fulda auf einem erheblich niedrigerem Niveau liegen.

\section{Abbildung 3.28: Einfluß der Telekommunikations-Standort- faktoren nach Regionen}

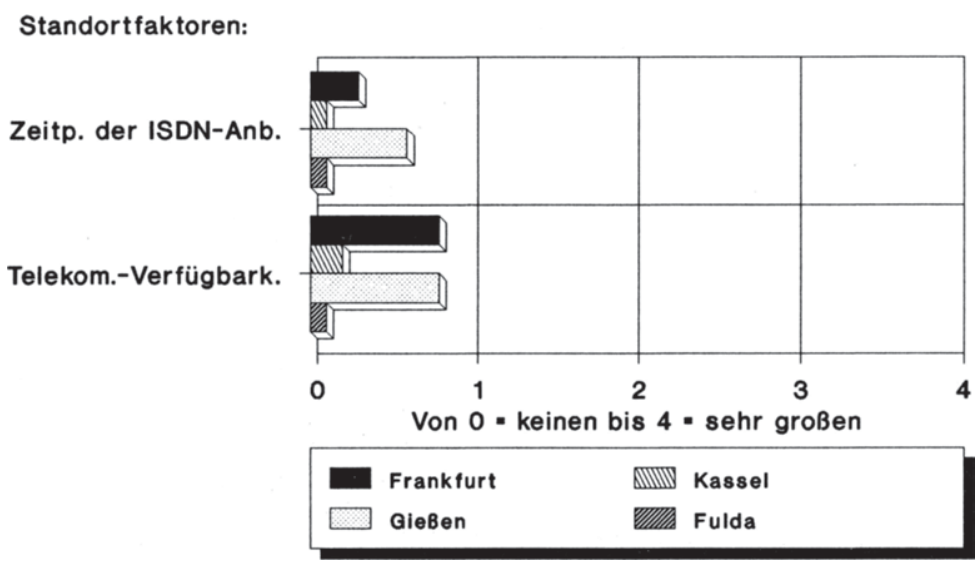

Als signifikant erweist sich auch der Zusammenhang der beiden Faktoren in Bezug zur Unternehmensgröße. Die Signifikanz beim ISDN-Anbindungszeitpunkt resultiert aus der stärkeren Bewertung des Faktors durch Kleinbetriebe, gegenüber den Betrieben mit mehr als 70 Beschäftigten.

Bei der Analyse bezüglich des Gründungszeitpunktes läßt sich bis zum Jahr 1988 keine Signifikanz nachweisen. Signifikant bis stark signifikant ist bei beiden Faktoren der Zeitraum ab 1988. Ab diesem zeitpunkt haben beide standortfaktoren signifikant an Bedeutung gewonnen. 
Nach Branchen untergliedert, liegt bei der Telekommunikations-Verfügbarkeit eine Signifikanz in Form einer schwachen Tendenz vor. Wie aus Abbildung 3.29 ersichtlich, ist die Rangfolge der Bewertung der beiden Faktoren ähnlich. Den größten Einfluß haben die Faktoren bei den Unternehmensberatungen und beim Maschinenbau, gefolgt von den Software/EDV-Firmen und dem Einzelhandel. Hieraus läßt sich zum Teil die regionale Signifikanz erklären, da in den vier Regionen die Branchen unterschiedliche Rückläufe hatten und somit den regionalen Trend verstärkten.

Abbildung 3.29: Einfluß der Telekommunikations-Standortfaktoren nach Branchen

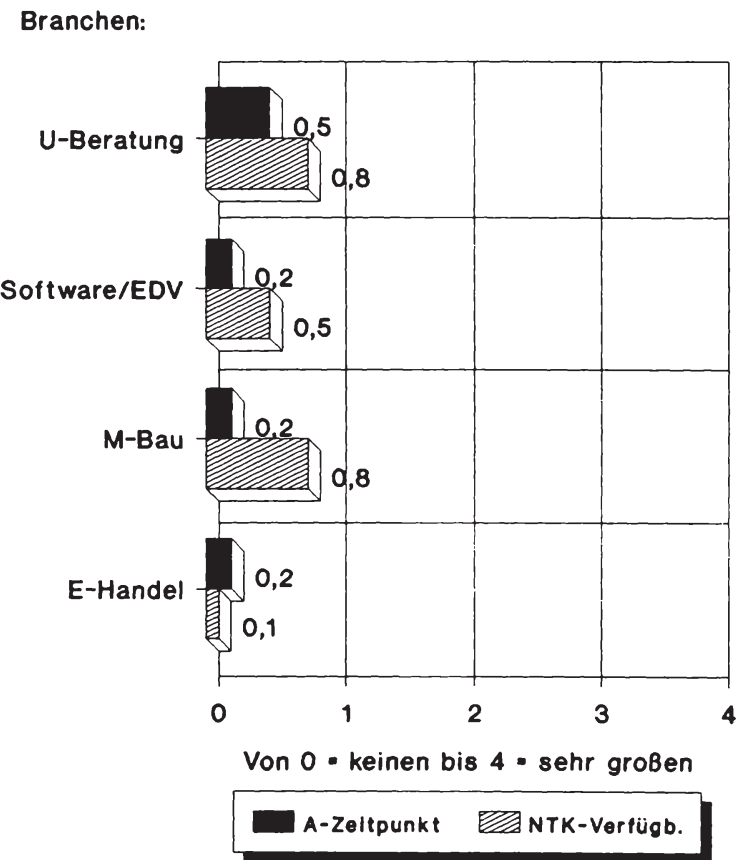




\subsubsection{Informationsquelien zur standortwahl}

Die Informationsquellen bezüglich des Unternehmenstandortes wurden in Frage 16 abgefragt. Von den fünf möglichen Antworten wurde mit $80 \%$ die persönliche Kenntnis angegeben. Auf Rang zwei liegt mit $10 \%$ die Entscheidung der Zentrale, gefolgt von Zeitungen und Büchern mit $6 \%$. Verbände und Gemeindevertreter liegen mit jeweils $2 \%$ auf dem vierten Rang. Diese Ergebnisse stehen im Zusammenhang mit der Angabe zu Frage 17, daß oftmals der Wohnstandort als Unternehmensstandort gewählt wird.

Abbildung 3.30: Informationsquellen zur standortwahl

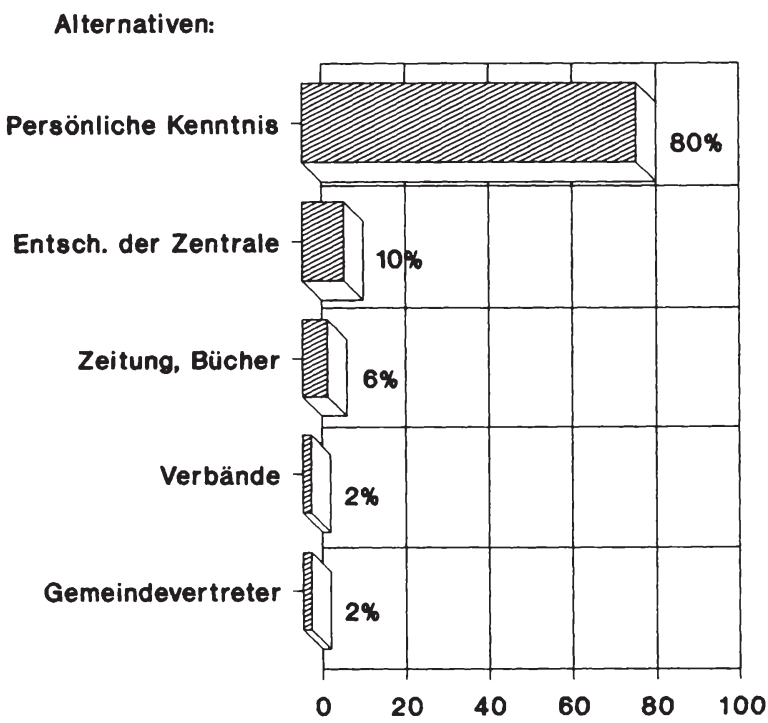

Antworten in \% der GG

Dntworten in \% der G 


\subsubsection{Telekommunikationsfördermaßnahmen}

Die Telekommunikationsfördermaßnahmen wurden grundsätzlich in drei Gruppen untergliedert:

1. Realtransfer in Form von kostenlosen

- Informationsdienstleistungen,

- Beratungsdienstleistungen und

- Ausbildungsdienstleistungen.

2. Finanzielle Anreize in Form von

- Subventionen für Neugründungen,

- Bereitstellung kostengünstiger Gewerbefläche sowie

- zinsgünstigen Darlehen oder Bürgschaften.

3. Anregung und Förderung von Rooperationen in Form

- gemeinsamer Unternehmensansiedlung in einem Gebäude (z.B. Möglichkeit eines gemeinsamen 'Telekommunikations-Gerätepools') und

- gemeinsamer Gründung einer Unternehmung durch mehrere Kooperationspartner, die als Teilhaber der Unternehmung auftreten.

Der Einfluß dieser Gruppen, in Bezug auf eine Standortaufwertung, wurde mit Frage 19 abgefragt. $\mathrm{Zu}$ jeder Fördermaßnahme sollte eine Bewertung von 0 gleich 'gar nicht' bis 4 gleich 'sehr stark' angegeben werden, die ausdrückt, inwieweit die jeweilige Maßnahme einen standort aufwertet.

Ausgehend von den Mittelwerten aller Antworten, wurden die finanziellen Anreize am höchsten bewertet. An zweiter Stel- 
le liegen die Realtransfers und an dritter Stelle die Anregung und Förderung von Kooperationen. Abbildung 3.31 zeigt, daß bei den finanziellen Anreizen die direkte Subventionierung von Neuinvestitionen, im Bereich neuer Informationsund Kommunikationstechniken, den ersten Rang einnimmt. Rang zwei wird, mit nur geringer Differenz zu Rang eins, von der Bereitstellung kostengünstiger Gewerbefläche und den zinsgünstigen Darlehen oder Bürgschaften belegt.

Abbildung 3.31: Einfluß der Fördermaßnahmen auf die standorteinschätzung

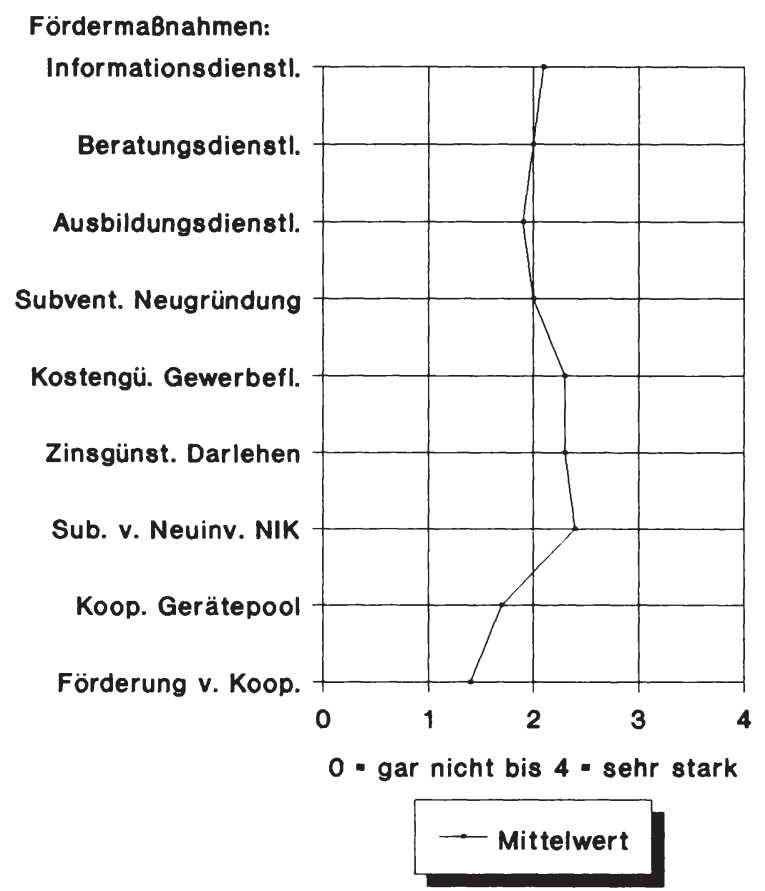

Auffallend ist die hohe Bewertung der drei Ausprägungen des Realtransfers. Wobei die Informationsdienstleistungen etwas höher und die Beratungsdienstleistungen gleich hoch wie die Subventionierung von Neugründungen eingeschätzt wird. Die 
Kooperationsförderung wird als weniger- bis mittelstark eingestuft und liegt damit deutlich hinter den beiden anderen Gruppen.

\section{Rorrelationen}

Die in Abbildung 3.32 dargestellte regionale Differenzierung hat, unter inferenzstatistischer Analyse62, keine signifikante Ausprägung. Die Bewertung der einzelnen Maßnahmen ist dennoch unterschiedlich.

Abbildung 3.32: Einfluß der Fördermaßnahmen auf die Standorteinschätzung nach Regionen

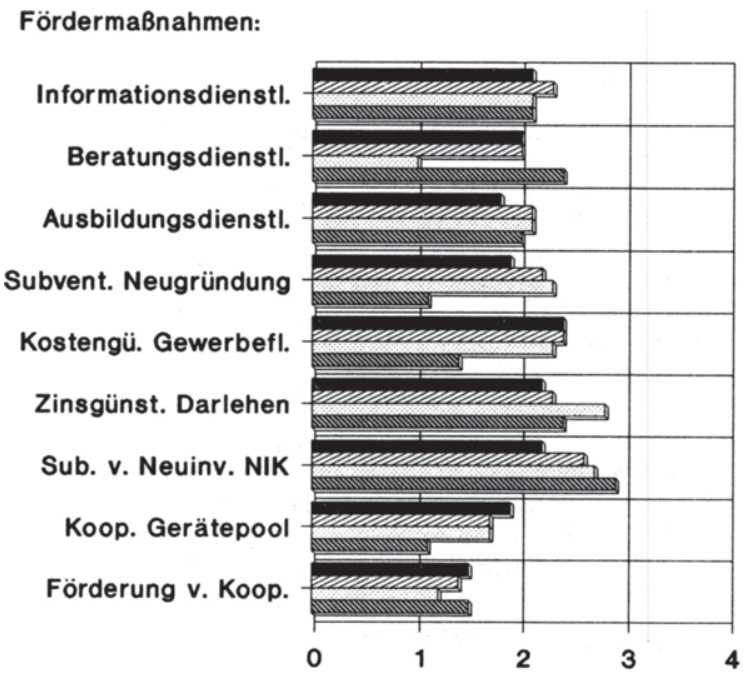

Skalenwerte von 0 - gar nicht bis 4 - sehr stark

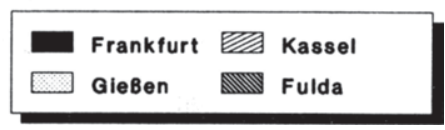

Frankfurt als Ballungsraum bewertet die ersten beiden Gruppen der Förderungen, im Verhältnis zu den anderen drei Re-

62 Vgl. Green, P.; Tull, D.: Methoden und Techniken der Marketingforschung, 4. Auflage, Stuttgart 1982, S. 410 ff. 
gionen, am schwächsten. Dagegen wird die dritte Gruppe eindeutig von Frankfurt angeführt. Kassel und Fulda führen leicht in Gruppe eins und Gießen in Gruppe zwei.

Als 'stark signifikant' erweist sich die Branchenzugehörigkeit und die EDV-Ausstattung. Wie aus Abbildung 3.33 ersichtlich, unterteilen sich die vier Branchen in zwei disparate Gruppen. Erstens die Unternehmensberatungen und Softwarefirmen und zweitens den Maschinenbau und Einzelhandel.

Abbildung 3.33: Einfluß der Fördermaßnahmen auf die Standorteinschätzung nach Branchen

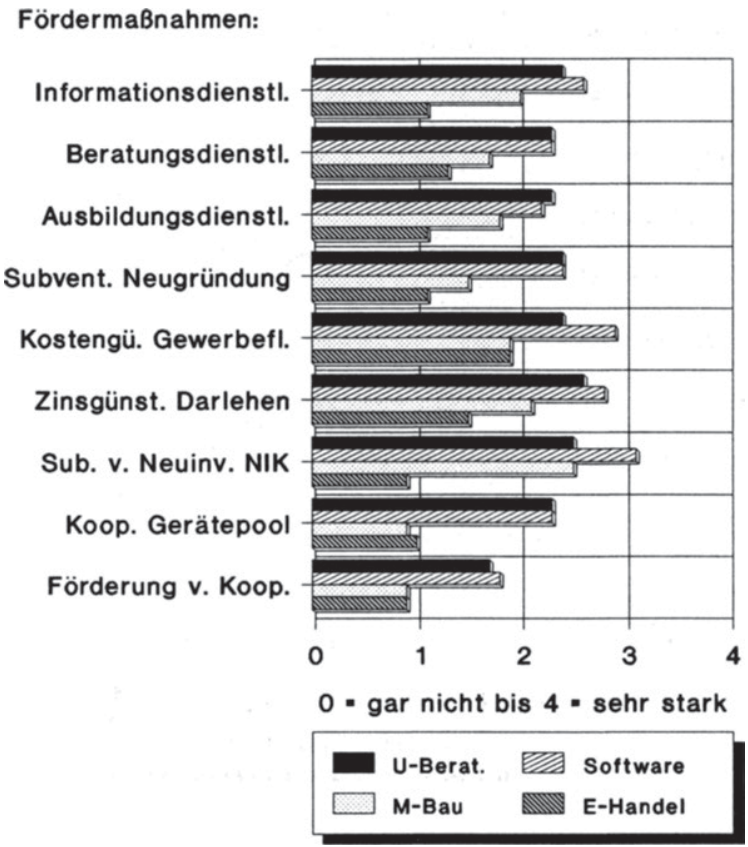

Bei der branchenbezogenen Analyse dominieren die Softwarefirmen und Unternehmensberatungen, die einheitlich die Förderungen höher einschätzen als die Maschinenbau- und Ein- 
zelhandelsunternehmen. Dies gilt im besonderen Maße für die Förderung von Kooperationen. Starke Signifikanz ließ sich inferenzstatistisch bei allen Förderungen, bezogen auf die Branchen, nachweisen. Bei den EDV-Anwendern ergaben sich ähnliche Werte, ausgenommen den 'Beratungsdienstleistungen' und der 'gemeinsamen Gründung einer Unternehmung', wo sich keine Signifikanz nachweisen ließ.

Ein signifikanter Zusammenhang konnte auch bei der Analyse bezüglich des Gründungszeitpunktes, inferenzstatistisch für die Realtransfers und die Anregung und Förderung von Kooperationen, nachgewiesen werden. Abbildung 3.34 zeigt, daß Unternehmen, die nach 1986 gegründet wurden, Fördermaßnahmen in allen angeführten Bereichen erheblich höher einschätzen als die bis 1985 gegründeten Unternehmen.

Abbildung 3.34: Einfluß der Fördermaßnahmen auf die Standorteinschätzung nach Gründungszeitpunkt

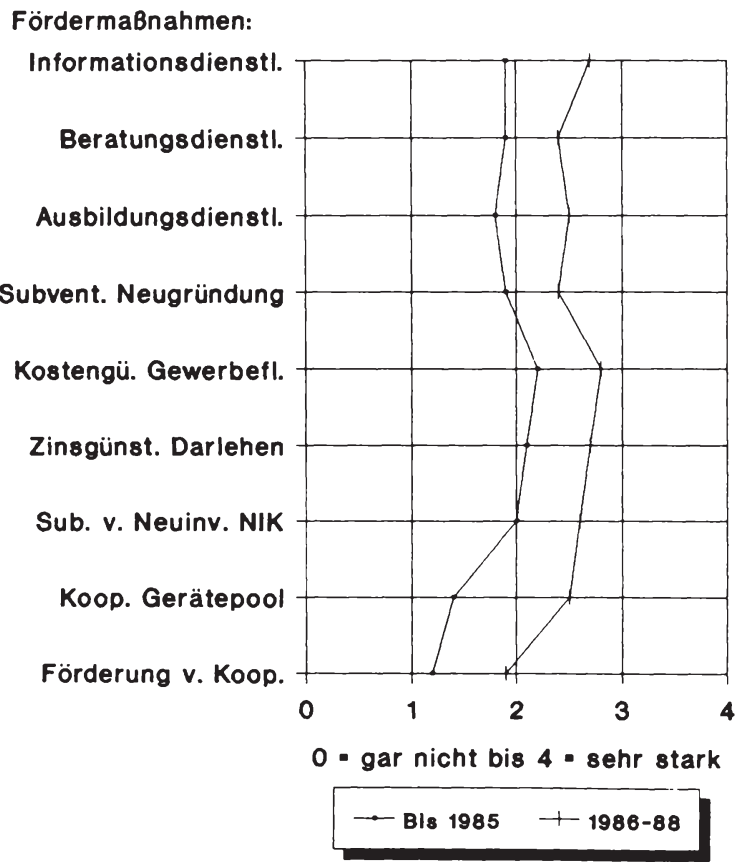




\subsubsection{Rommunikationsverhalten}

Die 'Kommunikationsintensität', die Kommunikationsarten und das Kommunikationssubstitutionspotential wurden mit den Fragen 10, 20 und 21 untersucht. Die Kommunikationsintensität wurde mittels der Fermeldegebühren und Endgerätekosten abgefragt. Das Kommunikationsverhalten der Unternehmen wurde bezüglich der am häufigsten verwendeten Kommunikationsarten ermittelt. Wobei das Kommunikationsverhalten grundsätzlich in einen internen und externen Bereich untergliedert wurde. Mit Frage 21 wurde zusätzlich abgefragt, inwieweit sich der persönliche Kontakt durch neue Telekommunikationstechniken substituieren läßt.

\subsubsection{Kommunikationsintensität}

Kommunikationsintensität als solche, ist eine Größe, die schwer zu quantifizieren ist. Als Indikator für die Kommunikationsintensität wurden erstens die Höhe der monatlichen Fernmeldegebühren und zweitens die Höhe der monatlichen Endgerätekosten gewählt.

Die monatlichen Fernmeldegebühren und die Endgerätekosten wurden in vier Klassen unterteilt. Erstens von 0,- DM bis unter 500,-DM, zweitens von 500,- DM bis unter 1000,- DM, drittens von 1000,- DM bis unter 5000,- DM und viertens von $5000,-$ DM und höher. Wie in Abbildung 3.35 ersichtlich, überwiegt mit $45 \%$ Anteil der Bereich 1000,- DM bis unter 5000,- DM. Lediglich 19\% liegen unter 500,- DM, und 14\% haben monatliche Gebühren von über 5000,- DM. Bei der Detecon-Studie vom Dezember $1987^{63}$ für Rheinland-Pfalz wurde

63 Vgl. Fölber, R.: Bedarfsuntersuchungen für die Fernmeldedienste in Rheinland-Pfalz, a.a.O., s. $49 \mathrm{ff}$. 
demgegenüber von 49,3\% der befragten Unternehmen eine monatliche Gebühr von unter 500,- DM angegeben.

Abbildung 3.35: Fernmeldegebühren in DM pro Monat

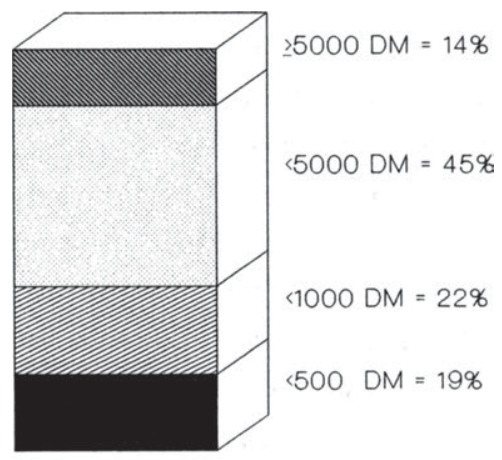

Die in Abbildung 3.36 dokumentierten Endgerätekosten pro Monat Jiegen erheblich unter den monatlichen Gebühren. $51 \%$ der Befragten haben Endgerätekosten von weniger als 500,DM im Monat und 20\% liegen über 500,- DM und unter 1000,DM.

Abbildung 3.36: Endgerätekosten in DM pro Monat

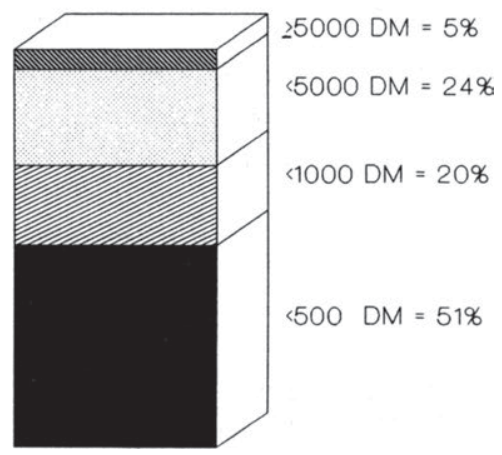




\section{Rorrelationen}

Signifikante zusammenhänge bezüglich der vier Regionen bestehen nicht. Korrelationen mit starker signifikanz liegen bei der Anzahl der Beschäftigten und dem onternehmensumsatz vor. Bei steigendem Umsatz, wie bei steigender Beschäftigtenzahl, erhöhen sich die monatlichen Fernmeldekosten. Bei der Analyse der vier Branchen ergibt sich eine starke signifikanz bezüglich der Kontrollgruppe. Die Unternehmensberatungen, Softwarefirmen und der Maschinenbau dokumentieren ein homogenes Verhalten gegenüber den Fernmeldekosten, wogegen der Einzelhandel erheblich geringere Kosten verzeichnet und somit weniger Kommunikation betreibt. Signifkant unterschiedlich verhalten sich EDV-Andwender gegenüber Nicht-EDV-Adoptoren. EDV-Anwender haben signifikant höhere Fernmeldekosten. Das gleiche gilt für Unternehmen die Forschungs- und Entwicklungsaktivitäten betreiben.

Die o.a. Korrelationen gelten in gleicher weise für die Endgerätekosten pro Monat.

Dominanteste Bestimmungsgröße der Kommunikationsintensität ist generell die Betriebsgröße, die durch weitere Bestimmungsgrößen ergänzt wird. Spehl/Messerig-Funk ${ }^{64}$ sehen eine enge Korrelation zwischen den Gebühren und dem Abhängigkeitsstatus des Betriebes und den Forschungs- und Entwicklungsaktivitäten. Dies deckt sich mit den 0.a. Ergebnissen, wobei zum Abhängigkeitsstatus ein signifikanter zusammenhang zwischen der eigenständigen Unternehmung und den Unternehmen mit mehreren Niederlassungen oder Zweigstellen besteht. Zusätzlich zu diesen Ergebnissen, sind im Rahmen dieser Arbeit branchentypische Abhängigkeiten festgestellt worden.

64 Vgl. Spehl, H.; Messerig-Funk, B.: Chancen und Probleme der wirtschaftlichen Nutzung der Telematik in einer ländlichen Region: Trier, a.a.0., S. $417 \mathrm{ff}$. 


\subsubsection{Interne und externe Rommunikationsarten}

Die Zusammensetzung des internen und externen Kommunikationsverhaltens wurde anhand von sieben Kommunikationsarten abgefragt, die jeweils intern und extern die summe von $100 \%$ ergeben sollten. Im internen Bereich dominiert eindeutig der persönliche Kontakt mit 54\%. Das Telefon liegt an zweiter stelle mit 16\%, gefolgt, mit Abstand, von der Datenübertragung mit $9 \%$, Telefax mit $8 \%$, Telex mit $7 \%$ und Brief mit $6 \%$. Auffallend ist, daß Btx für interne Kommunikation von den befragten Unternehmen nicht verwendet wird.

Abbildung 3.37: Kommunikationsarten intern

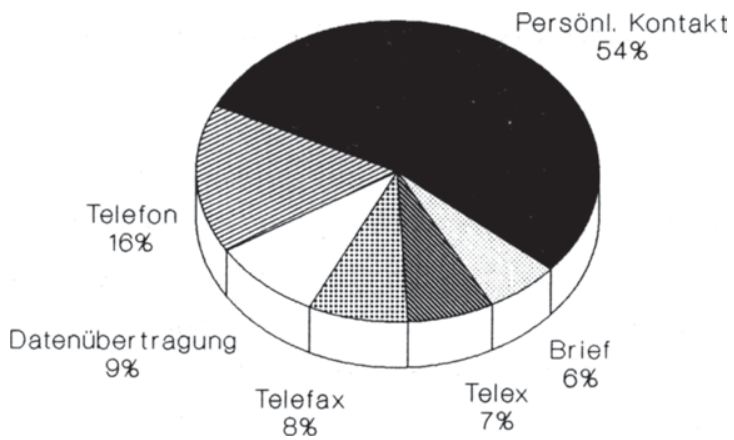

Beim externen Kommunikationsverhalten ergiebt sich eine Verschiebung $z u$ Gunsten des Telefons, das mit $35 \%$ an erster stelle steht, dicht gefolgt vom persönlichen Kontakt mit 30\%. Die restliche Kommunikation wird durch Brief (13\%), Telefax (10\%), Datenübertragung (7\%), Telex (3\%) und Btx (3\%) geführt. Auffallend ist der relativ hohe Anteil an Telefax gegenüber Telex und Btx, der bei der externen Kommunikation fast an den Briefanteil heranreicht. 


\section{Abbildung 3.38: Kommunikationsarten extern}

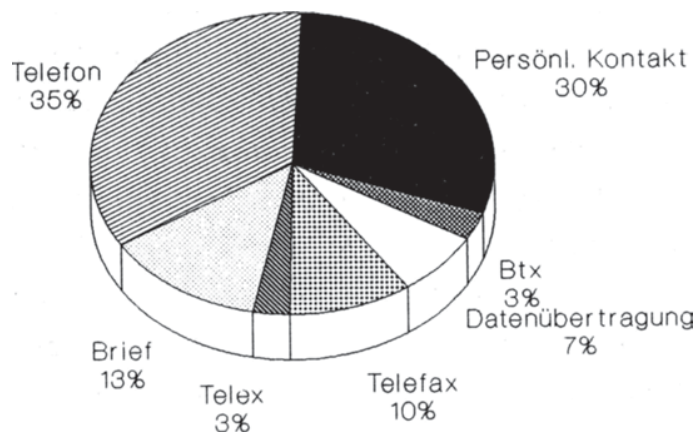

\subsubsection{Bubstitutionspotential persönlicher Rontakte}

Inwieweit sich persönliche Kontakte durch neue Telekommunikationstechniken substituieren lassen, wurde mit Frage 21 abgefragt. $\mathrm{Zu}$ jedem der angegebenen Telekommunikationsdienste sollte auf einer skala von $0 \%$ bis $100 \%$ jeweils das inund externe substitutionspotential angegeben werden. Eine alternative Antwortmöglichkeit war das statement 'Persönliche Kontakte lassen sich nicht durch neue Telekommunikationstechniken ersetzen'.

Abbildung 3.39 zeigt die differenzierte Einschätzung des Substitutionspotentials. Im interen Bereich werden erheblich geringere potentiale angesetzt, als im externen Bereich. Die im externen Bereich angebenen Werte liegen beim ISDN-Telefon und beim ISDN-Telefax mit jeweils $30 \%$ am höchsten, gefolgt vom Bildtelefon (26\%) und der Viedeokonferenz (17\%). Mit Abstand folgt das ISDN-Btx (9\%), das ISDN-Teletex $(4 \%)$ und das ISDN-Telex (5\%). Die interne Ein- 
schätzung entspricht in etwa der externen, jedoch auf entsprechend niedrigerem Niveau.

\section{Abbildung 3.39: Substitutionspotential persönlicher Kon- takte durch neue Telekommunikationstech- niken}

\section{Kommunikationsart:}

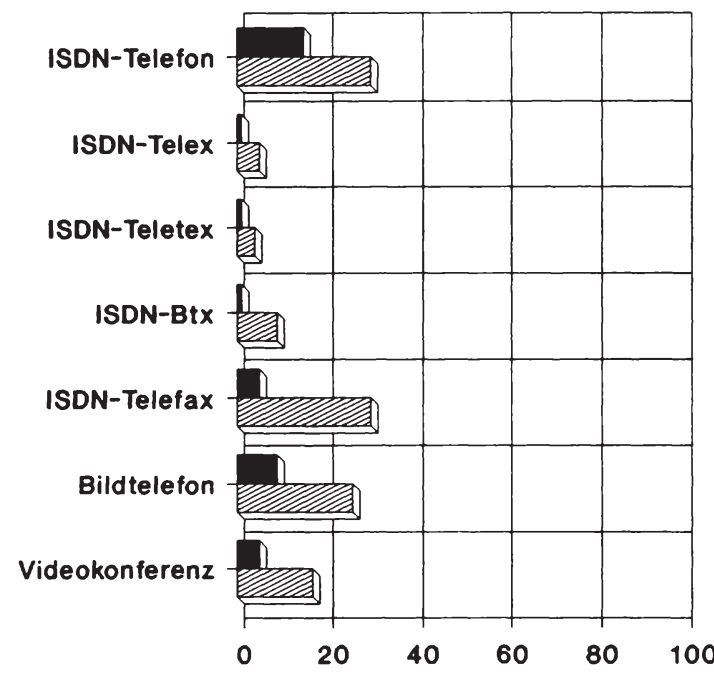

Werteskala von 0 bis $100 \%$ je Dienst

\section{intern extern}

Die, im Bereich ISDN-Telefon, ISDN-Telefax und Bildtelefon, hohen Einschätzung von etwa $30 \%$ substitutionspotential, werden durch die Zusatzfrage, ob eine substitution von persönlichen Kontakten überhaupt möglich ist, stark relativiert. Grundsätzlich sehen, wie aus Abbildung 3.40 ersichtlich, nur $35 \%$ der befragten Unternehmen eine Substitutionsmöglichkeit. $65 \%$ der Unternehmen sind der Meinung, daß sich 
der persönliche Kontakt, auch durch neue Telekommunikationstechniken, nicht substituieren läßt. Signifkante Unterschiede bezüglich dieser Einschätzung konnten nicht festgestellt werden.

Abbildung 3.40: Können persönliche Kontakte durch neue Telekommunikationstechniken ersetzt werden?

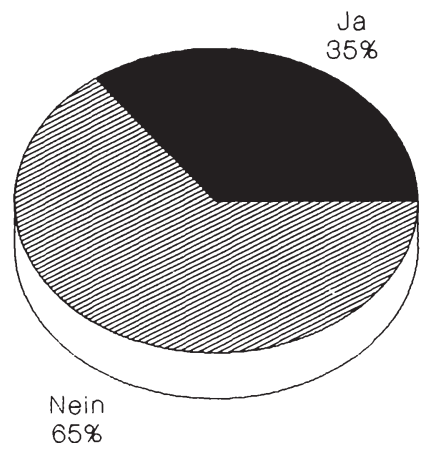




\subsection{Interpretation und Zusammenfassung der Ergebnisse der empirischen Untersuchung}

\subsubsection{Telekommunikation als standortfaktor}

\subsubsection{EinfluB der Telekommunikation auf die standortwahl}

Die Mehrheit der befragten Unternehmen gab an, daß der ISDN-Anbindungszeitpunkt und die Telekommunikations-Verfügbarkeit keinen Einfluß auf die standortwahl hatten. Lediglich bei Unternehmen die ab 1988 gegründet wurden, konnte ein Anstieg der Bedeutung der Telekommunikation als standortfaktor festgestellt werden. Der standortfaktor 'Anbindung an Straßen/Autobahn' beispielsweise, ist im Verhältnis zum 'ISDN-Anbindungszeitpunkt' achtmal wichtiger. Bringt man die untersuchten standortfaktoren in eine Rangskala, so nimmt der ISDN-Anbindungszeitpunkt den letzten Platz ein. Einen Hinweis auf die Ursache dieser schlechten Ergebnisse gibt die hohe Anzahl der Antworten 'nicht zu beantworten' und die 'missing values'65. Sie deuten auf ein Informationsdefizit bezüglich der neuen telekommunikativen Techniken hin.

Signifikante regionale Disparitäten bei der Einschätzung des Einflusses der Telekommunikation auf die standortwahl bestehen zwischen den Regionen Frankfurt - Kassel und GieBen - Fulda, wobei Gießen und Frankfurt auf einem etwa gleich hohen Niveau liegen. Kassel und Fulda bewegen sich auf einem erheblich niedrigeren Niveau. 66

65 missing values = Überhaupt keine Angabe zur Frage gemacht.

66 Siehe Abbildung 3.28. 
Die Arbeitshypothese 'Telekommunikation ist ein standortfaktor für Klein- und Mittelbetriebe' kann als Ergebnis der vorliegenden empirischen Untersuchung nicht bestätigt werden. Die Interpretation der empirischen Daten führt zu der Erkenntnis, daß Unternehmen primär andere Anforderungen an einen neuen standort stellen. Neben den traditionellen standortfaktoren, nimmt die Telekommunikation eine untergeordnete Rolle ein und erst wenn diese Faktoren erfüllt sind, kann der Faktor neue telekommunikative Techniken relevant werden.

\subsubsection{8tandortaufwertung durch Förderung neuer Telekom- munikationstechniken}

Mit nur geringen regionalen Disparitäten wurden die Fördermaßnahmen bewertet. Den finanziellen Anreizen, die am höchsten eingestuft wurden, folgten mit geringen Abstand die Realtransfers. Eine relativ schlechte Bewertung erhielt die Anregung und Förderung von Kooperationen. Die am höchsten eingeschätzte Einzelmaßnahme im Bereich der finanziellen Anreize war die 'Subventionierung von Neuinvestitionen im Bereich neuer telekommunikativer Techniken', mit einer Bewertung der potentiellen standortaufwertung zwischen 'mittel-stark' und 'stark'. Im Bereich der Realtransfers war die Einstufung der 'Informationsdienstleistungen' mit 'mittel-stark' am höchsten.

Bei der Analyse nach Branchen liegen die Unternehmensberatungen und Software-Firmen in der Bewertung erheblich höher. Der Maschinenbau und der Einzelhandel zeigen hier nur wenig Interesse. Dagegen weisen neugegründete Firmen ein erheblich größeres Interesse an Förderungen aus. Sie stufen Fördermaßnahmen zwischen 0.5 und 1.0 Punkten 67 höher ein

67 Ausgehend von einer Skala von 0 bis 4 Punkten. 
als die anderen Unternehmen. Auffallend ist auch hier die sehr hohe Einstufung der Informationsdienstleistungen mit 2.7 Punkten.

Trotz der hohen Einstufung der Fördermaßnahmen liegen die ausschlaggebenden Kriterien für eine standortwahl, besonders bei kleineren Unternehmensberatungen und Software-Firmen, oftmals in den persönlichen Präferenzen des Gründers. Der Wohnort oder studienort entspricht bei etlichen Neugründungen dem Unternehmensstandort. Erst nach Erfüllung der präferierten Standortanforderungen des Unternehmensgründers, kann der Faktor Telekommunikationsförderung überhaupt relevant werden. Dann wird die Förderung gerne 'mitgenommen' und kann zwischen 'gleichwertigen' Standorten den Ausschlag geben. 68

Berücksichtigt man zusätzlich die Ergebnisse aus Kapitel 3.3.1.1., so stellt sich die Frage, ob eine unmittelbare Förderung der Telekommunikation mit dem ziel einer standortlenkung als zweckmäßig angesehen werden kann, oder ob nicht eine verstärkte Konzentration auf die unterschiedlichen Formen des Realtranfers, im besonderen auf die Informationsdienstleistungen, das endogene Entwicklungspotential der jeweiligen Region aktivieren könnte und somit letztendlich die Region für Neuansiedlungen attraktiver wird.

\subsubsection{Adoption neuer telekommunikativer Techniken}

\subsubsection{Regionales Adoptionsverhalten}

Regionale Disparitäten bezüglich der Adoption neuer Telekommunikationsdienste bestehen nicht durchgängig. Bei den

$68 \mathrm{Vgl}$. Brösse, U.: Ziele in der Regionalpolitik und in der Raumordnungspolitik, a.a.0., s. 141. 
bereits länger angebotenen Diensten existieren geringe Adoptionsunterschiede. Bei den Datenübertragungsdiensten und den jüngeren Telekommunikationsdiensten existieren jedoch stärkere Disparitäten, wobei Frankfurt und Gießen die erheblich höheren Adoptionsraten erzielen als Kassel und Fulda.

Die Untersuchung von Fritsch kommt hier zu ähnlichen Ergebnissen. 69 "Eine durchgängige Variation der regionalen Adoptionsraten entsprechend dem Rang des jeweiligen Standortes in der Raumhierarchie kann nicht festgestellt werden." 70 Ausgenommen des Telefaxdienstes, läßt sich für die Adoption der neuen Telekommunikationsdienste kein signifikanter standorteinfluß feststellen.71 Regionale Unterschiede in der Adoption basieren weitestgehend auf den unterschiedlichen Charakteristiken der jeweiligen Betriebe. 72 Diese Ergebnisse werden auch von Müdespacher 73 in seiner schweizerischen Untersuchung über die räumliche Diffusion von Telematik-Anwendungen bestätigt.

$69 \mathrm{Vgl.}$ Fritsch, M.: Räumliche Unterschiede der TelematikAdoption in Industriebetrieben der Bundesrepublik Deutschland, in: Akademie für Raumforschung und Landesplanung (Hrsg.): Räumliche Wirkungen der Telematik, Forschungs und Sitzungsberichte Band 169, a.a.0., S. $327 \mathrm{f}$.

70 Ebenda S. 327 .

71 Bei der Interpretation der Ergebnisse von Fritsch muß berücksichtigt werden, daß sich die Untersuchung auf Industriebetriebe mit Fertigung am standort beschränkte. Die Einbeziehung von typischen Frühadoptoren wie der Bereich Banken, Versicherungen und Verwaltungszentralen, die vorrangig in den stadtzentren angesiedelt sind, könnte hier $\mathrm{zu}$ anderen Ergebnissen führen.

72 Vgl. Fritsch, M.: Räumliche Unterschiede der TelematikAdoption in Industriebetrieben der Bundesrepublik Deutschland, a.a.0., S. 327 f.

73 Müdespacher, A.: Informationstechnologie, räumliche Difiusionsprozesse und Adoptionsverhalten der schweizerischen wirtschaft, TH zürich, Institut für orts-, Regional- und Landesplanung (Hrsg.), Zürich 1986. 
Nimmt man die Rücklaufquote als zusätzlichen Indikator für das Interesse an neuen telekommunikativen Techniken, so ergibt sich ein Gefälle zwischen den ausgewählten Regionstypen. Frankfurt liegt mit $27 \%$ an der spitze, gefolgt von Gießen mit $23 \%$, Kassel mit $16 \%$ und Fulda mit $8 \%$.

Demgegenüber stehen die Aussagen bei der Einschätzung der Bedeutung der Telekommunikationsdienste. Hier wird von Kassel und Fulda den Diensten teilweise eine höhere Bedeutung zugemessen als von Frankfurt und Gießen. Diese grundsätzlich positive Einschätzung der neuen Dienste wird zusätzlich durch die Akzeptierung höherer Endgerätepreise dokumentiert. Kassel und Gießen liegen durchschnitlich um DM 500,- über den angegebenen Werten der Region Frankfurt. 74

\subsubsection{Einflußgrößen der Adoption}

Die obigen Ergebnisse bezüglich des regionalen Adoptionsverhaltens deuten darauf hin, daß die Region als solche keinen statistisch signifikanten Beitrag zur Erklärung der regional unterschiedlichen Adoption von Telekommunikationsdiensten leistet, sondern andere Merkmale wie die regionale wirtschaftsstruktur, die Unternehmensgröße, die Forschungsund Entwicklungsaktivitäten und die EDV-Anwendung für eine Adoption telekommunikativer Techniken von Bedeutung sind.

Die Ergebnisse der eigenen Untersuchung, bezüglich des Adoptionsverhaltens neuer Telekommunikationsdienste, werden von den Untersuchungen von Fritsch 75 für Industriebetriebe

74 Die regionalen Differenzen in der Preisvorstellung bezüglich der Endgerätekosten können jedoch auch Ausdruck mangelnder Information über die aktuellen Marktpreise sein.

75 Vgl. Fritsch, M.: Räumliche Unterschiede der TelematikAdoption in Industriebetrieben der Bundesrepublik Deutschland, a.a.0., s. $301 \mathrm{f}$. 
mit Fertigung in ausgewählten Regionen der Bundesrepublik Deutschland, Spehl/Messerig-Funk ${ }^{76}$ für die Region Trier im Verarbeitenden Gewerbe und von der Deutschen Telepost Consulting $\mathrm{GmbH}^{77}$ für Unternehmen und öffentliche Verwaltungen in Rheinland-Pfalz bestätigt. Als Bestimmungsgrößen der Adoption neuer Telekommunikationsdienste ergeben sich folgende Annahmen:

- Größere Unternehmen,

- Unternehem mit eigenen Forschungs- und Entwicklungstätigkeiten und

- Unternehmen mit EDV-Anwendung

adoptieren neue Telekommunikationsdienste häufiger als andere Unternehmen und

- der jeweilige Bekanntheitsgrad und Informationsstand bestimmt nachhaltig den Einsatz und die Planungsabsichten bezüglich einer Adoption neuer Telekommunikationsdienste. 78

\subsubsection{Adoptionshemmnisse}

Als wesentliche Gründe für den Nichteinsatz neuer telekommunikativer Dienste wurden der fehlende Nutzen, die Kostenbelastung, mangelnde Information und die ungewisse Diensteentwicklung angegeben. Die empirischen Unteruchungen von

76 Vgl. Spehl, H.; Messerig-Funk, B.: Chancen und Probleme der wirtschaftichen Nutzung der Telematik in einer

ländlichen Region: Trier, a.a.0., S. $417 \mathrm{ff}$.

77 Vgl. Schnöring, T.: Wirkungen der Telekommunikationsentwicklung auf Anwender, a.a.0., S. 48 .

$78 \mathrm{Vgl}$. auch ebenda, S. 48 . 
Spehl/Meeserig-Funk ${ }^{79}$ und der Deutschen Telepost Consulting $\mathrm{GmbH}^{80}$ bestätigen diese Adoptionshemmisse.

Der am häufigsten angegebene Rejektionsgrund in dieser Untersuchung war 'kein Nutzen für den Betrieb' und deutet auf ein entsprechendes Informationsdefizit 81 bezüglich der Einsatzmöglichkeiten neuer telekommunikativer Techniken hin. Die weiteren Rejektionsgründe sind, nach den Antworthäufigkeiten geordnet, $z u$ hohe Gebühren, zu hohe Anfangsinvestitionen, zu geringe Flächendeckung, unklare Gebührenstruktur, unzureichende Informationen, Qualifikationsproblematik, Endgeräteproblematik und als letzter Punkt kein ISDNAnschluß. Das bereits o.a. Informationsdefizit wird besonders von der Region Fulda angegeben, die, im Vergleich zu Kassel, ein viermal höheres Informationsdefizit hat.

Die mangelnde Information, besonders bei den neuen telekommunikativen Diensten der Deutschen Bundespost, bezieht sich jedoch nicht nur auf den Nutzen für den Betrieb, sondern auch auf die Dienste als solche, was durch den geringen Bekanntheitsgrad einzelner Dienste dokumentiert wird. 82

\subsubsection{Thesen zur Telekommunikation}

Ziel der empirischen Untersuchung war eine Überprüfung der Arbeitshypothese und der abgeleiteten Unterhypothesen aus Kapitel 3.1.2.

79 Vgl. Spehl, H.; Messerig-Funk, B.: Chancen und Probleme der wirtschaftlichen Nutzung der Telematik in einer ländlichen Region: Trier, a.a.0., S. 427.

$80 \mathrm{Vgl}$. Fölber, R.: Bedarfsuntersuchungen für die Fernmeldedienste in Rheinland-Pfalz, a.a.0., S. 73.

$81 \mathrm{Vgl}$. ebenda, S. 123.

82 Siehe Kapitel 3.2.2.1. 
Die Arbeitshypothese 'Telekommunikation ist ein standortfaktor für klein- und Mittelbetriebe' muß verworfen werden.

Die abgeleiteten Unterhypothesen bezüglich des Adoptionsverhaltens wurden anhand der empirischen Ergebnisse überprüft und zusätzlich um weitere Bestimmungsgrößen ergänzt. Als Indikatoren für die Adoptionsintensität wurden der 'heutige Einsatz', der 'geplante Einsatz' und der 'Bekanntheitsgrad' der Telekommunikationsdienste gewählt.

Die Überprüfung der abgeleiteten Hypothesen ergab:

1. Ein homogenes 'Agglomeration $<->$ strukturschwache Regionen' Gefälle quantitativer Art zwischen den verschiedenen Regionen und der Adoption neuer telekommunikativer Techniken existiert nicht.

2. Die Charakteristika der Standorte haben keinen substantiellen Einfluß auf das qualitative Adoptionsverhalten der Anwender.

3. Zwischen der Größe eines Unternehmens und seinem Adoptionsverhalten existiert ein signifikanter Zusammenhang. Größere Unternehmen sind typische Frühadoptoren, wogegen kleinere Unternehmen zu den Spätadoptoren zählen. ${ }^{83}$

4. Das Vorhandensein von Forschungs - und Entwicklungsaktivitäten fördert die Adoption neuer Telekommunikationstechniken.

5. Einzelbetriebe weisen gegenüber Unternehmen mit mehreren Betriebsstätten eine geringere Adoptionsrate auf.

83 Die Investitions- und Betriebskosten der Telekommunikationsdienste haben einen evidenten Einfluß in Bezug auf das temporäre, quantitative und qualitative Adoptionsverhalten. 
6. Ein homogener Bedarf nach Telekommunikationsdiensten existiert nicht. Der nachgefragte Dienst resultiert primär aus dem Kommunikationsverhalten der jeweiligen Branche. 84

7. Die Nachfrage nach neuen telekommunikativen Diensten wird nicht durch die reine Verfügbarkeit der Dienste geprägt, sondern durch die Kenntnis und den Informationsstand möglicher Anwendungen und Kosten. 85

8. Mikro-bzw. Personal-Computer-Anwender zählen zu den Frühadoptoren.

Weitere im Zusammenhang stehende Thesen lauten:

9. Neugegründete Unternehmen weisen gegenüber länger bestehenden Unternehmen eine höhere Adoptionsrate auf. Dies gilt im besonderen bezüglich der jüngeren Telekommunikationsdienste.

10. Es besteht ein 'Zentrum $\leftrightarrow->$ strukturschwache Regionen' Gefälle bezüglich des Informationsstandes über neue Telekommunikationsdienste, deren Nutzung und deren Kosten.

84 Räumlich unterschiedliche Adoptionsraten lassen sich weitgehend auf unterschiedliche Charakteristiken der jeweils ansässigen Betriebe (Größe, Unternehmenszugehörigkeit, FuE-Tätigkeiten usw.) zurückführen. In Frankfurt ist z.B. der Bereich Unternehmensberatung und in Fulda der Bereich Maschinenbau stärker vertreten.

$85 \mathrm{Vgl}$. auch Fölber, R.: Bedarfsuntersuchungen für die Fernmeldedienste in Rheinland-Pfalz, a.a.0, S. 117, Ewers, H.; Fritsch, M.: Die räumliche Verbreitung von computergestützten Techniken in der Bundesrepublik Deutschland, in: Böventer von, E. (Hrsg.): Regionale Beschäftigung und Technologieentwicklung, Berlin 1989, S. $108 \mathrm{ff}$ und Spehl, H.; Messerig-Funk, B.: Chancen und Probleme der wirtschaftlichen Nutzung der Telematik in einer ländlichen Region: Trier, a.a.0., S. 427 . 
11. Strukturschwache und ländliche Regionen sind im Gegensatz zu den Agglomerationen bereit, einen höheren Preis für die Telekommunikationsendgeräte zu bezahlen.

12. Bei Unternehmensneugründungen nimmt der Standortfaktor Telekommunikation einen höheren stellenwert ein als bisher.

13. Die Rejektionsgründe werden primär von der mangelnden Nutzeneinschätzung und nicht von den Kosten oder sonstigen Faktoren bestimmt.

Die abgeleiteten Hypothesen Nr. 1 und 2 wurden durch die Ergebnisse der empirschen Erhebung nicht bestätigt und somit abgelehnt. Die Hypothesen $\mathrm{Nr}$. 3-8 wurden durch die Erhebung bestätigt und somit angenommen. Die Hypothesen 9-13 leiten sich aus den zusätzlich aufgetretenen Korrelationen und signifikanten Zusammenhängen der Erhebung ab. 
4. Beurteilung der Telekommunikationsförderung als regionalpolitisches steuerungsinstrument

\subsection{Grundsätzliche Überlegungen zur Akzeptanz neuer Tech- niken}

Nach der Prognose der britischen Marktforschungs- und Technologie-Beratungsorganisation BIS Mackintosh und der Eastern Management, wird im Bereich der ISDN-Technik bis 1996 weltweit ein Absatzmarkt für Telekommunikationssysteme, -endgeräte und -dienste in Höhe von 41,5 Mrd. Dollar erschlossen sein. ${ }^{1}$ Diese Entwicklung ist von der Akzeptanz der potentiellen ISDN-Nutzer abhängig. Eine gewisse 'Vorsicht', mit der potentielle Anwender auf eine neue Technologie mit ungeprüften Vorteilen zugehen, konnte durch die Untersuchung eindeutig nachgewiesen werden. ${ }^{2}$

Teilweise fühlen sich Menschen von den neuen Informationsund Kommunikationstechnologien bedroht. Im Vordergrund stehen Ängste bezüglich der Arbeitsplatzsicherheit und der Auswirkungen auf die Privatsphäre. Diese Ängste basieren u.a. auf der 'Datensammelwut' von Unternehmen mit geringem ethischen Verantwortungsbewußtsein gegenüber ihren Mitarbeitern. ${ }^{3}$ Diese 'Datensammelwut' läßt sich aus einer Variante des Parkinsonschen Gesetzes, das auf die computerwelt uminterpretiert wurde, ableiten: 4

$1 \mathrm{Vgl}$. o.V.: Künftige ISDN-Anwender müssen überzeugt werden, in: VDI-Nachrichten vom 11.3.1988, Düsseldorf 1988.

$2 \mathrm{Vgl}$. ebenda.

3 Vgl. Bodendorf, F.; Lüthi, A.: Neue Informations- und Kommunikationstechnologien, a.a.0., S. 356.

$4 \mathrm{Vgl.}$ Miller, A.: 'Computer and Privacy', in: Hoffmann, W.; Mills Moore, J. (Eds.): Ethics and the Managment of Computer Technology. Proceedings of the Fourth National Conference on Business Ethics. Cambridge, Massachusetts 1982 , S. 93-114. 
Wer einen Computer hat, wird Mittel und Wege finden, die gesamte speicherkapazität mit Daten $z u$ füllen und bei Erreichung der Kapazitätsgrenze einen neuen leistungsfähigeren computer anschaffen.

Die Akzeptanz gegenüber neuen Informations- und Kommunikationstechniken hängt von der Information über die realen Vor- und Nachteile sowie von der unmittelbaren Erfahrung ab. Für die Realisierung von Akzeptanzsteigerungen sind frühzeitige Aufklärung und Einbeziehung sowie Maßnahmen der Aus- und Weiterbildung erforderlich. 5 Da die Informationsund Kommunikationstechnik eine Großtechnologie darstellt, die mit Wachstumsargumenten in der öffentlichkeit begründet wird, stellt die zu erwartetende Akzeptanzproblematik einen wichtigen Engpaßfaktor für die zukünftige Entwicklung des ISDN dar. 6

Momentan werden potentiellen ISDN-Anwendern mit dem Hinweis auf zukünftig günstigere Gebührenstrukturen für Telefax und Datenkommunikation umworben. Diese günstigen Gebührenstrukturen kommen jedoch erst bei entsprechender ISDN-Anschlußhäufigkeit zum tragen und können, von der nicht nur durch wirtschaftliche Erwägungen gelenkten Deutschen Bundespost Telekom, auch wieder geändert werden. 7

Im folgenden wird die Kritik an den Netzausbauplänen der Deutschen Bundespost und die Akzeptanz neuer Techniken im regionalen Vergleich dargestellt.

5 Vgl. Klaus-Stöhner, U.; Zabel, J.: Möglichkeiten und Anwendungsbarrieren für den Einsatz neuer Informations- und Kommunikationstechniken in einer peripheren Region: Fallstudie Waldeck-Frankenberg, a.a.0., S. 414.

6 Vgl. Hoffmann, M.; Siegele, L.: Kurzschluß bei der Post, in: Die Zeit Nr. 5 vom 27. Januar 1989, S. 37.

$7 \mathrm{Vgl}$. Reon, K.: ISDN - Alles aus einer Dose mit begrenztem Nutzen, in: Frankfurter Allgemeine Zeitung $\mathrm{Nr} .89$ vom 17. April 1990, S. T 1. 


\subsubsection{Rritik an den Netzausbauplänen der Deutschen Bun- despost}

Die ökonomisch orientierte Kritik an den Netzausbauplänen konstatiert, daß der grundsätzlich richtige Ausbauplan der Deutschen Bundespost zu langsam realisiert wird. Dadurch entgehen den deutschen Herstellern internationale Marktchancen und national wird die kritsche schwelle im Diffusionsprozeß nicht überschritten. 8

Die auf soziale Risiken konzentrierte Kritik an den Netzausbauplänen geht von einem differenzierten Ansatz aus, der sich in drei Hauptbereiche untergliedern läßt:

- die technische Komplexität,

- die Verwundbarkeit und

- den Datenschutz. 9

Das Schmalband-ISDN und das Universalnetz stellen Rechnernetze dar, in die die momentan existierenden spezialnetze, entsprechend der Ausbaustufe, integriert werden. Mit zunehmender Integration der Spezialnetze steigt jedoch die Komplexität des Rechnernetzes und das Fehlerrisiko beziehungsweise die Gefahr eines Totalausfalls. In der Diskussion bezüglich der Risiken von Hochtechnologien wird, entgegen dem ISDN-Konzept, die Fehlerfreundlichkeit und Entkoppelung von Systemen gefordert. 10

$8 \mathrm{Vgl}$. Kubicek, H.; Mettler-Meibom, B.: Alternative Entwicklungspfade der Telekommunikationspolitik, B46-47, S. 35 .

$9 \mathrm{Vgl}$. ebenda.

10 Vgl. z.B. Ulrich, E.; Weizäcker, von C.: Fehlerfreundlichkeit als evolutionäres Prinzip, in: Wechselwirkungen, Nr. 29, 1986, S. $12 \mathrm{ff}$. 
Die erhöhte Verwundbarkeit von hochkomplexen und zentralisierten systemen gegenüber physischer Gewalt und SoftwareManipulationen, ist quasi systemimmanent. Speziell aus der Möglichkeit alle Nutzungen zu protokollieren, erwächst die Gefahr, die Protokolle als Mittel der vorbeugenden Gefahrenabwehr oder der nachträglichen Identifizierung zu verwenden. 11

Personenbezogene Daten dürfen, nach dem Recht auf informationelle selbstbestimmung, nur im erforderlichen Umfang gespeichert werden und die speicherung, Verarbeitung und weitergabe muß für die Betroffenen transparent sein. Die Digitalisierung der Vermittlungstechnik in Form speicherprogrammierter Vermittlungsrechner führt dazu, daß Verbindungsdaten wie Datum, Uhrzeit, Gebühreneinheiten und Zielnummer gespeichert werden und zukünftig zwecks Erstellung differenzierter Gebührenrechnungen Verwendung finden. Im letztendlich geplanten Universalnetz werden zusätzlich Daten, wie beispielsweise die Nutzung von Fernseh- und Hörfunkprogrammen, gespeichert. 12

Die Kritik an den technischen Kontrollmöglichkeiten des ISDN ergibt sich nicht erst, wenn die Absicht einer Personenüberwachung unterstellt werden kann. Nach den Erfahrungen von Datenschutzbeauftragen, kann davon ausgegangen werden, daß Dateien, die für einen bestimmten Zweck errichtet werden, Begehrlichkeiten an anderen stellen wecken, denen im Lauf der Zeit teilweise entsprochen wird. Die Kommunikation der Bürger gilt in dem Moment als eingeschränkt, in dem das Wissen um die Möglichkeit einer technische Überwa-

11 Vgl. Hoffmann, M.; Lütge, G.: Blind für die Risiken, in: Die Zeit $\mathrm{Nr} .6$ vom 3. Februar 1989, S. 24.

12 Vgl. Kubicek, H.; Mettler-Meibom, B.: Alternative Entwicklungspfade der Telekommunikationspolitik, a.a.o., s. 37 . 
chung zu einer Kommunikation quasi mit der 'Schere im Kopf' führt. 13

Die Angst vor dem 'gläsernen Menschen' kann zu einem wichtigen Adoptionshemmis für die neuen telekommunikativen Techniken werden. Die Belange der potentiellen Anwender sollten deshalb von der Deutschen Bundespost bei der Entwicklung neuer Techniken berücksichtigt und dokumentiert werden. Datenschutz und Datensicherheit könnten sich zu einem zukünftigen Qualitätsmerkmal herausbilden, das für die weitere Diffusion der neuen Dienste mitentscheidend wird. 14

\subsubsection{Regionale und soziostrukturelle Disparitäten der Akzeptanz neuer Techniken}

Die folgenden Erkenntnisse über die soziale Akzeptanz neuer Techniken im regionalen Vergleich resultieren aus der Umfrageforschung der Bundesforschungsanstalt für Landeskunde und Raumordnung zur Betroffenheit und zu Einstellungen gegenüber 'neuen Techniken', die sich unter anderem nach dem Regionstyp, der großräumigen Lage und den sozialstrukturellen Merkmalen untergliedert. 15

Im Rahmen der Erhebung wurden die konkreten Erwartungen bezüglich der wirkung neuer Techniken analysiert. Bei der

13 Vgl. exemplarisch Garstka, H.: Schutz von Persönlichkeitsrechten bei der Nutzung neuer Medien, in: Kilian, W. (Hrsg.): Neue Medien für die Individualkommunikation, München 1984 , S. 79-85.

14 Schnöring, T.: Wirkungen der Telekommunikationsentwicklung auf Anwender, a.a.0., S. 109.

15 Vgl. Böltken, F.: Soziale Akzeptanz neuer Techniken im regionalen Vergleich, in: Informationen zur RaumentwickIung, Heft 4, 1989, S. 245. 
Frage zur Einstellung gegenüber neuen Techniken standen zwei Antwortvorgaben zur Auswahl: 16

- Die neuen Techniken führen langfristig zu immer größerer Arbeitslosigkeit auf allen Gebieten. Gleichbedeutend für Technik als Bedrohung.

- Die neuen Techniken schaffen auf lange sicht neue Berufschancen und Arbeitsplätze. Geichbedeutend für Technik als chance.

\section{Analyse nach Regionstypen}

Die Untersuchung ergab eine Abhängigkeit der Einstellung gegenüber den neuen Techniken von der Einbindung ins Erwerbsleben. Nichterwerbstätige fühlen sich stärker bedroht als Erwerbstätige. Nennenswerte Unterschiede nach der siedlungsstrukturellen Lage existieren nicht. Wie aus Tabelle 4.1 ersichtlich, beurteilen die Erwerbstätigen, besonders

Tabelle 4.1: Einschätzung neuer Techniken als 'Chance' nach Regionstyp und Erwerbstätigkeit in 17

\begin{tabular}{|l|c|c|}
\hline Regionstyp & Nicht-Erwerbstätige & Erwerbstätige \\
\hline $\begin{array}{l}\text { Strukturstarke Verdich- } \\
\text { tungsregionen }\end{array}$ & $23 \%$ & 378 \\
\hline $\begin{array}{l}\text { Altindustrialisierte Ver- } \\
\text { dichtungsregionen }\end{array}$ & $23 \%$ & 438 \\
\hline $\begin{array}{l}\text { Regionen mit Verdichtungs- } \\
\text { ansätzen }\end{array}$ & 228 & 358 \\
\hline Ländliche Regionen & 248 & 328 \\
\hline
\end{tabular}

in den altindustrialisierten Verdichtungsregionen, die Chancen der neuen Techniken tendenziell positiv. Wobei die

$16 \mathrm{Vgl}$. ebenda.

17 In Anlehnung an Böltken, F.: Soziale Akzeptanz neuer Techniken im regionalen Vergleich, a.a.O., s. 248. 
positiven Einschätzungen der Erwerbstätigen in den altindustrialisierten Regionen auf Hoffnungen und nicht, wie in den strukturstarken Regionen, auf Erfahrungen mit den neuen Techniken basiert. 18

\section{Analyse im Nord-süd-Vergleich}

Im Nord-Süd-Vergleich der Einstellungen zu den neuen Techniken, wie in Tabelle 4.2 dargestellt, ergibt sich ein suidNord-Gefälle der Technikakzeptanz. 40\% der Erwerbstätigen im Süden ${ }^{19}$ sehen eine Chance durch die neuen Techniken, ähnlich auch die Erwerbstätigen in der Mitte 20 mit $37 \%$. Im Norden ${ }^{21}$ sind es lediglich 29\%, die eine Chance durch neue Techniken sehen. Bei den Nichterwerbstätigen zeigt sich ein ähniiches Gefälle. Lediglich 13\% der Nicht-Erwerbstätigen betrachten die neuen Techniken als eine Chance, gegenüber $25 \%$ in der Mitte und $27 \%$ im Süden.

Tabelle 4.2: Einschätzung neuer Techniken als 'Chance' nach Erwerbstätigkeit im Nord-Süd-Vergleich in $\% 22$

\begin{tabular}{|l|c|c|}
\hline Großräumige Lage & Nicht-Erwerbstätige & Erwerbstätige \\
\hline \hline Nord & $13 \%$ & $29 \%$ \\
\hline Mitte & $25 \%$ & $37 \%$ \\
\hline Süd & $27 \%$ & $40 \%$ \\
\hline
\end{tabular}

Dieses Süd-Nord-Gefälle der höheren Technikakzeptanz läßt sich nicht durch ein technologisches süd-Nord-Gefällen er-

$18 \mathrm{Vgl}$. ebenda S. 249.

19 Bayern und Baden-württemberg.

20 Nordrhein-Westfalen, Hessen, Rheinland-Pfalz und Saarland.

21 Hamburg, Bremen, Niedersachsen und Schleswig-Holstein.

22 In Anlehnung an Böltken, F.: Soziale Akzeptanz neuer Techniken im regionalen Vergleich, a.a.O., S. 250. 
klären. "Vielmehr scheinen hier zwei Faktoren ausschlaggebend: Einmal die Erfahrung struktureller stabilität, die wegen - oder trotz - des Einsatzes neuer Techniken vermittelt wird, zum anderen die gängigen stereotypen vom fortschrittlichen Süden, die eine von Erfahrung unabhängige Technikakzeptanz bewirken." 23

\section{Analyse nach sozialstrukturellen Merkmalen}

Von besonderem Interesse ist der in Tabelle 4.3 dargestellte Zusammenhang zwischen sozialstrukturellen Merkmalen, nach der stellung im Beruf und der Akzeptanz neuer Techniken, unter dem Aspekt, daß die Erhebung des Verfassers im Bereich Klein- und Mittelbetriebe, in denen primär Selbständige, einfache und mittlere Angestellte und Arbeiter $^{24}$ vertreten sind, anzusiedeln ist.

Tabelle 4.3: Akzeptanz neuer Techniken nach sozialstrukturellen Merkmalen in ${ }^{25}$

\begin{tabular}{|c|c|c|c|}
\hline \multicolumn{2}{|c|}{ Stellung im Beruf } & Bedrohung & Chance \\
\hline \multirow{3}{*}{ ARBEITER } & ungelernte Arbeiter & 828 & 188 \\
\hline & Facharbeiter & 748 & 268 \\
\hline & Vorarbeiter/Meister & 688 & 328 \\
\hline \multirow{3}{*}{ ANGESTELLTE } & einfache Angestellte & 718 & 298 \\
\hline & mittlere Angestellte & 658 & 358 \\
\hline & höhere Angestellte & 488 & 528 \\
\hline \multicolumn{2}{|l|}{ SELBSTÄNDIGE } & 668 & 348 \\
\hline
\end{tabular}

23 Ebenda S. 251.

24 Wobei unter Arbeitern ungelernte Arbeiter, Facharbeiter, Vorarbeiter und Meister verstanden werden.

25 In Anlehnung an Böltken, F.: Soziale Akzeptanz neuer Techniken im regionalen Vergleich, a.a.O., S. 252 . 
Wie aus Tabelle 4.3 ersichtlich, sinkt mit dem beruflichen Status auch die Akzeptanz neuer Techniken bzw. die Hoffnung auf positive Arbeitsplatzauswirkungen. Vorarbeiter/Meister, mittlere Angestellte und Selbständige beurteilen die Chancen auf etwa gleichem Niveau zu 32-35\% positiv. Die einzige Gruppe, die die Chancen zu über $50 \%$ als positiv einschätzt, sind die höheren Angestellten. Insgesamt werden von der überwiegende Mehrheit der Befragten die neuen Techniken als Bedrohung angesehen.

Zusammenfassend kann von einem Süd-Nord-Gefälle der Technikakzeptanz gesprochen werden, das nicht auf entsprechend konkreten Erfahrungen, soziostrukturellen Merkmalen oder Qualifikationsmerkmalen basiert, sondern vor allem bei Nichtbetroffenheit reproduziert wird. Das stereotyp und Image eines fortschrittlichen, innovationsfreudigen südens, das bestimmten Regionen Prosperität und Fortschrittlichkeit und anderen Deprivation und Rückständigkeit zuweist, kann für die Regionalentwicklung von erheblicher Bedeutung sein. Dies gilt im besonderen unter dem Aspekt, daß der Faktor Image einer Region bei standortentscheidungen von Unternehmen und Haushalten zunehmend an Bedeutung gewinnt. 26

\subsection{Beurteilung der Telekommunikationsfördermaßnahmen}

\subsubsection{Beurteilung der Telekommunikation als standortfak- tor}

Vor der Beurteilung der Telekommunikationsfördermaßnahmen, unter der Perspektive der empirischen Erhebung des Verfassers und in Bezug auf das Bundesland Hessen, wird kurz auf

$26 \mathrm{Vgl}$. ebenda S. 257. 
den Aspekt der Telekommunikations-Verfügbarkeit als standortfaktor eingegegangen.

wie in Kapitel 3.3.1.1. dargelegt, führt die Interpretation der empirischen Daten zu der Erkenntnis, daß die Unternehmen primär andere Anforderungen, d.h. nicht die Telekommunikations-Verfügbarkeit, an einen neuen standort stellen. Neben den traditionellen standortfaktoren, nimmt die Telekommunikation eine untergeordnete Rolle ein, die, wenn überhaupt, erst nach der Erfüllung der traditionellen Faktoren zur Geltung kommt.

Eine Neudefinition der standortkriterien erscheint für die absehbare zukunft als nicht realistisch. Dies basiert zum Teil auf der existierenden Akzeptanzproblematik der traditionsorientierten, innovationsabgeneigten Mentalität von Unternehmen und deren Beschäftigten. Ausgehend von der ubiquitären Informationsversorgung durch die Massenmedien, erscheint vielfach eine weitere Verbesserung der Informationslandschaft als nicht mehr möglich. 27 "Viele Benutzer neuer Medien bevorzugen eher ihren individuell aufgebauten Informationspool, da er ihren speziellen Bedürfnissen eher entspricht und setzen die Medien nur zu ganz spezifischen Zwecken ein, z.B. zum Bestellen."28

Einen wesentlichen Faktor stellt die nach wie vor existierende Dominanz der folgenden traditionellen standortkriterien dar. Dies sind

27 Vgl. exemplarisch Langenbucher, W.: Individuum und Haushalt als Informationssucher - Chancen für Bildschirmtext, in: Witte, E. (Hrsg.): Telekommunikation für den Menschen, Berlin 1980, S. 131.

28 Quentmeier-Vieregge, R.; Storbeck, D.: Möglichkeiten und Hindernisse der Telematik am Beispiel eines Mittelzentrums: Herford, in: Akademie für Raumforschung und Landesplanung (Hrsg.): Räumliche wirkungen der Telematik, a.a.0., s. 465 . 
- die Anbindung an Straßen und Autobahnen,

- die Nähe zum Absatzmarkt,

- die Anbindung an die Bundesbahn und

- das Image und Umfeld. 29

Ausgehend von diesen vier dominantesten standortfaktoren ${ }^{30}$, sind zusätzlich die persönlichen Präferenzen des Gründers ausschlaggebend. Der Wohn- und studienort des Gründers stellt oftmals den Unternehmenstandort dar.

Verbesserte Kommunikationsmöglichkeiten können, wie o.a., keinen standortwechsel an einen eventuell preiswerteren, peripheren standort motivieren. Die räumliche Erreichbarkeit stellt oftmals eine 'Conditio sine qua non' für die als notwendig angesehenen face-to-face Kontakte dar, mit deren Hilfe die Wettbewerbsposition wesentlich gesteigert werden kann. 31

\subsubsection{Beurteilung der finanziellen Anreize}

Ausgehend von der empirischen Erhebung des Verfassers, kommen den finanziellen Anreizen in Form von Subventionen, unter dem Aspekt einer standortaufwertung, die größte Bedeutung zu. 32 An erster stelle der Fördermaßnahmen steht die Subventionierung von Neuinvestitionen, an zweiter stelle die Gewährung zingsgünstiger Darlehen und an dritter stelle die Bereitstellung kostengünstiger Gewerbefläche.

29 In der Reihenfolge ihrer Bedeutung.

30 Siehe Abbildung 3.25.

31 Vgl. Lauschmann, E.: Grundlagen einer Theorie der Regionalpolitik, a.a.0., s. $56 \mathrm{f}$.

32 Siehe Abbildung 3.31. 
Eine explizite Förderung telekommunikativer Techniken erscheint aufgrund der existierenden bundes- und landesweiten Förderungen, wie unter Kapitel 2.3.1.1.1. dargestellt, nicht notwendig, da die Einführung telekommunikativer Techniken $z u$ den organisatorischen Innovationen zählt und somit förderwürdig ist. Zusätzlich ist es fraglich, ob eine explizite subventionierung der Telekommunikation tatsächlich von den strukturschwachen Regionen absorbiert würde, oder lediglich eine weitere Begünstigung der ohnehin strukturstarken Agglomeration Rhein-Main bewirkt. 33

Ausgehend von der Vielfalt der bundes- und landesweit angebotenen Investitionshilfen, ist eine Verbesserung der Information über die öffentlichen Förderprogramme, im besonderen der speziellen Branchen- und Technikförderprogramme, notwendig. Vorgesehen ist eine Anbindung an ein DatenbankSystem in Form einer 'DASTI - Datei der staatlichen Investitionshilfen', deren Pflege und Aktualisierung von der Hessichen Landesentwicklungs- und Treuhandgesellschaft übernommen werden kann. Über dieses Informationssystem können dann Sparkassen, Banken, Kammern, wirtschaftsförderungsinstitutionen usw. online auf die Datei der staatlichen Investitionshilfen zugreifen. 34

\subsubsection{Beurteilung der Realtransfers}

Die Telekommunikationsfördermaßnahmen in Form von Realtransfers wurden nur minimal schlechter bewertet als die finanziellen Anreize, wobei die Informationsdienstleistun-

$33 \mathrm{Vgl}$. Hamm, W.: Milliardenstrom in die Ballungsgebiete, in: Frankfurter Allgemeine Zeitung $\mathrm{Nr} .169$ vom 25. Juli 1989 , S. 9 .

$34 \mathrm{Vgl}$. Hessisches Ministerium für Wirtschaft und Technik: strukturpolitischer Bericht für Mittelhessen, a.a.o., s. 70 . 
gen am höchsten eingeschätzt wurden, gefolgt von den kostenlosen Beratungs- und Ausbildungsdienstleistungen. Im folgenden werden die Fördermaßnahmen im einzelnen vorgestellt und beurteilt.

\section{Individuelle Beratungen}

Ein explizite Förderung von individuellen Beratungen im Bereich telekommunikativer Techniken erscheint aufgrund der existierenden bundes- und landesweiten Förderungen, wie unter Kapitel 2.3.1.1.2. dargestellt, nicht notwendig. 35

\section{Information und Beratung durch die Deutsche Bundespost}

Bestandteil des Marketingkonzepts der Deutsche Bundespost ist die kostenlose Information und Beratung über die angebotenen oder kurz vor der Einführung stehenden Telekommunikationsdienste. Neben den Informationsangeboten in Form von Broschüren werden Anwenderseminare durchgeführt. Desweiteren existieren Informationszentren zur Information und Beratung der jungen Generation und der kleinen und mittleren Unternehmen.

Das Informations- und Beratungsangebot der Deutschen Bundespost ist jedoch auf die nachfragestarken städte konzentriert, wie beispielsweise Frankfurt und Kassel. Desweiteren basieren die Informationen primär auf den technischen Details der Dienste und nicht auf den potentielien Anwendungseffekten oder Kosten-Nutzen-Überlegungen. Ansatzpunkte einer Verbesserung des Informations- und Beratungsangebots der Deutschen Bundespost bilden die landesweite Expansion der Informations- und Beratungsleistung und die Erweiterung dieser Leistung um die Anwendungskomponente. Als Schwerpunkt müssen Informations- und Beratungsangebote in strukturschwachen Regionen verstärkt auf- und ausgebaut werden.

35 Beispielsweise werden die Beratungen des bayerischen Telekommunikationszentrums mit $90 \%$ durch das bayerische Technologieberatungsprogramm gefördert. 


\section{Innovations- und Technologieberatungsstellen}

Die Innovations- und Technologieberatungsstellen in Hessen bieten Unterstützung in Form von Kontaktanbahnung, Information und Beratung. Abgesehen davon, daß die Beratungsstellen relativ unbekannt sind, konzentrieren sie sich, wie in Abbildung 2.3 dargestellt, auf Großstädte, mit einem insgesamt starken Süd-Nord-Gefälle. In Nordhessen sind lediglich drei Innovations- und Technologieberatungsstellen in Kassel vertreten und für den Bereich osthessen ist nur eine Beratungsstelle in Fulda eingerichtet. Berücksichtigt man zusätzlich, daß beispielsweise die Innovations- und Technologie-Beratungsstelle bei der Industrie- und Handelskammer Frankfurt aus drei Mitarbeitern ${ }^{36}$ besteht, so wird die Notwendigkeit einer Intensivierung der Förderung, besonders in den struturschwachen Regionen, evident.

\section{Einschlägige Informations- und Beratungseinrichtungen}

Einschlägige Informations- und Beratungseinrichtungen im Bereich der Informations- und Kommunikationstechniken stellen beispielsweise das Informationstechnik-Zentrum in Köln, das ostfriesische Informationstechnikzentrum und das bayerische Telekommunikationszentrum dar. In Hessen existiert kein derartiges Zentrum.

Besonders interessant stellt sich für die Gruppe der kleinen und mittleren Unternehmen die Möglichkeit dar, im bayerischen zentrum herstellerneutral über die Einsatzmöglichkeiten von Telekommunikationsdiensten und über mögliche Arbeitsplatzkonfigurationen informiert $\mathrm{zu}$ werden und anhand der Dauerausstellung von Telekommunikationsgeräten, die zu Modellarbeitsplätzen konfiguriert sind, sich Gestaltungsideen für das eigene Unternehmen abzuleiten. Hierbei wird neben der technischen Beratung auch eine betriebswirtschaftliche Auswertung vorgenommen. Die hohe Anzahl der Be-

$36 \mathrm{Vgl}$. O.V.: Informationen aus Datenbanken - Beschaffung Beratung, Frankfurt 1989, S. 6 . 
ratungen durch das bayerische Telekommunikationszentrum dokumentieren die Akzeptanz gegenüber dem Zentrum.

Ein solches Informationszentrum ist in Hessen nicht vorhanden, könnte jedoch beispielsweise in den Industrie- und Handelskammern eingerichtet werden und zum Teil, wie in Bayern, über Landesförderprogramme finanziert werden.

\section{Qualifizierungsoffensive 'Neue Technologien'}

Die Qualifizierungsoffensive 'Neue Technologien' des Landes Hessen stellt eine gezielte Berücksichtigung der neuen Technologien im Bereich der betrieblichen Erstausbildung und der Erwachsenen-Weiterbildung dar, die mittels der existierenden Berufsbildungsinfrastruktur nicht befriedigt werden kann. 37 Die grundsätzlich positiv zu beurteilende Qualifizierungsoffensive befindet sich jedoch zur zeit noch größtenteils in der Planungs- und Koordinationsphase. Bereits realisierte Förderungen konzentrieren sich auf Ausstattungsinvestitionen für neue Technologien in bereits existierende überbetriebliche Berufsbildungseinrichtungen. Eingeleitet sind zusätzlich Modellversuche zur Ermittlung des Weiterbildungsbedarfs, neuer Lehrgangsinhalte, der Möglichkeiten des Weiterbildungsverbundes und von neuen Lehrgangsbausteinen im Bereich der Aus- und Fortbildung.

Erforderlich sind weitere Maßnahmen bezüglich

- der Ausbildung der Ausbilder und Ausbilderinnen,

- der Neuordnung von Berufsbildern,

- der Entwicklung qualifizierter Weiterbildungsberufe,

- der Entwicklung von Lehrgangsbausteinen und Schulungsmaterial und

- der Förderung überbetrieblicher und außerbetrieblicher

$37 \mathrm{Vgl}$. Hessiches Ministerium für Wirtschaft und Technik: Strukturpolitischer Bericht für Nord- und Osthessen, Beiträge zur Wirtschaftspolitik in Hessen, Heft 15, Wiesbaden 1988 , S. 88 ff. 
Schulungsmaßnahmen für kleine und mittlere Unternehmen. ${ }^{38}$

Hierbei erscheint grundsätzlich eine stärkere Ausrichtung der Qualifizierungsoffensive in Richtung Telematik als notwendig. Auch bei diesem Instrument ist darauf $\mathrm{zu}$ achten, daß verstärkt die peripheren Gebiete angesprochen werden.

\subsubsection{Beurteilung der Anregung und Förderung von Koopera- tionen}

Die Anregung und Förderung von Kooperationen, wie beispielsweise in Form von Technologie- und Gründerzentren realisiert, wurde bei der Befragung am schlechtesten bewertet. Ein Teil der Begründung dieses Ergebnisses liegt in der Tatsache, daß in Hessen Technologie- und Gründerzentren relativ gering vertreten sind. In Hessen existieren lediglich drei Zentren, im Vergleich $\mathrm{zu}$ bundesweit 73 Zentren. 39

Bei der Beurteilung der Technologie- und Gründerzentren muß berücksichtigt werden, daß abschließende Aussagen über die noch sehr jungen Zentren nicht möglich sind. Besonders Prognosen über die Entwicklung von Technologie- und Gründerzentren in den neuen Problemregionen sind derzeit kaum möglich.

Gegen die Technologie- und Gründerzentren in wirtschaftsschwachen Peripherregionen spricht vor allem, neben der vergleichsweise hohen Kostenbelastung besonders kleiner und finanzschwacher Trägergemeinden, 40 daß speziell technolo-

$38 \mathrm{Vgl}$. Hessisches Ministerium für Wirtschaft und Technik: strukturpolitischer Bericht für Mittelhessen, a.a.0., s. 92.

39 Stand: März 1989.

$40 \mathrm{Vgl}$. Schrumpf, H.: Technologieparks als Instrument der kommunalen Wirtschaftsförderung, Bochum 1984, S. 95. 
gieorientierte Unternehmen extrem standorttreu sind und nur in Ausnahmefällen eine Anwerbung von Unternehmen aus anderen Regionen als erfolgreich angesehen werden dürfte. Dies gilt im besonderen für Gründungsunternehmen, sodaß kaum Verlagerungen aus anderen Raumordnungskategorien $\mathrm{zu}$ erwarten sind, oder sie geschehen auf Kosten ländlicher Regionen. 41

Zusätzlich gehen von den Technologie- und Gründerzentren keinesfalls kurzfristig, jedoch auch langfristig, keine nennenswerten direkten Beschäftigungseffekte aus. ${ }^{42}$ Der wirtschaftliche wert technologieorientierter Jung- und Kleinstunternehmen drückt sich primär in technologiepolitischen und imagebezogenen Dimensionen aus und nicht in Beschäftigungszahlen.

Unter diesen Aspekten erscheint eine verstärkte Förderung von Technologie- und Gründerzentren in Hessen als fragwürdig.

\subsubsection{Beurteilung infrastrukturpolitischer Maßnahmen}

Die Deutsche Bundespost bietet, im Vergleich zum Ausland, neue telekommunikative Dienste relativ schnell flächendekkend an. Nach der Analyse des Verfassers, stellt das Angebot von Telekommunikationsdiensten durch die Deutsche Bundespost gegenwärtig keinen Engpaßfaktor dar. Bezüglich des ISDN-Ausbaus besteht demzufolge momentan kein Handlungsbe-

41 Vgl. Schröder, K.: 'Technologieparks' in Niedersachsen Eine Analyse der Ziele, Konzepte und Implementierung, Göttingen 1985, S. 116 ff.

$42 \mathrm{Vgl}$ Sternberg, R.: Technologie- und Gründerzentren als Instrument kommunaler Wirtschaftsförderung, a.a.o., s. 293. 
darf für die städte, die bis 1991 im Knotenvermittlungsbereich angeschlossen werden sollen. ${ }^{43}$

Infrastrukturpolitische Maßnahmen erscheinen jedoch für Kommunen notwendig, deren ISDN-Anschlußzeitpunkt erheblich später geplant ist, was bei einer weiteren Verzögerung des Gesamtausbaus zunehmend an Relevanz gewinnt. Hier erscheint eine Abstimmung mit den jeweiligen oberpostdirektionen als notwendig, um für die Übergangszeit, bis zur vollen Einführung, entsprechend Einfluß auf die Reihenfolge der Zurverfügungstellung von Übergangslösungen (Fremdanschaltungen) innerhalb einer Region, ausüben zu können. 44

Von regionalpolitisch größerer Relevanz ist die Gebührenregelung, die sowohl aus regional- als auch ordnungspolitischer sicht kritisiert wird. Periphere Räume fühlen sich durch die derzeitige Tarifgestaltung benachteiligt, da sie anteilmäßig mehr Ferngespräche führen und die dort ansässigen Betriebe und Haushalte entsprechend höhere Kosten tragen. Aus raumordnungspolitischer sicht ist, unter diesem Aspekt, eine Verbilligung der Fernmeldegebühren bis zu einer Entfernung von $100 \mathrm{~km}$ anzustreben.

Aufgrund des Kostenanteils der Fernmeldegebühren an den Gesamtkosten der Unternehmen, sind die Kommunikationskosten jedoch für die überwiegende Zahl der Unternehmen als standortfaktor von geringer Bedeutung. Andererseits können hohe Telekommunikationskosten im ländlichen Raum, obwohl sie ökonomisch nicht relevant sind, ein emotionales Anwendungshindernis für die Diffusion neuer Dienste darstellen. 45

43 Siehe Abbildung 2.5..

$44 \mathrm{Vgl}$. Lange, S.: Neue Aufgaben für die kommunale Wirtschaftspolitik, a.a.0., S. 515.

$45 \mathrm{Vgl}$. Kordey, N.: Gebührenpolitik im Fernmeldewesen und Raumordnung, a.a.0., S. 224. 
Relativiert werden die Überlegungen bezüglich der Tarifstruktur im Fernmeldewesen durch die erheblich höhere Úbertragungsgeschwindigkeit im ISDN, die die Bedeutung der Entfernungskomponente innerhalb der Gebührenregelung reduziert.

Zusammenfassend verbleiben der regionalen Infrastrukturpolitik somit als notwendige Einflußbereiche:

- die Sicherstellung der Bereitstellung von provisorischen Anschlußmöglichkeiten für peripher gelegene Nutzer und

- die Maßnahmen zur Einführung einer Tarifstruktur, die mit den regional-bzw. raumordnungspolitischen $\mathrm{zielen}$ vereinbar sind.

\subsection{Telekommunikationsförderung im Ausland}

\subsubsection{Informationstechnologische Lokalzentren als Instru- ment der Regionalpolitik in Norwegen}

Im September 1985 wurde in Vemdalen, einem schwedischen Dorf mit 800 Einwohnern, die erste Telestube eröffnet. Mit finanzieller Unterstützung der öffentlichen Hand, hat ein Privatmann ein Büro mit Teletex, Telefax, Bildschirmtext und Personal-Computern ausgestattet und zusätzlich einen Unterrichtsraum, eine Teeküche und einen Aufenthaltsraum eingerichtet. Bereits nach ca. zwei Jahren hat ein Fünftel der Dorfbevölkerung an Computerkursen teilgenommen, werden mittels Telearbeit schreib- und Übersetzungsdienste geleistet und die Telekommunikationstechniken der Telestube von den lokalen Kleinstbetrieben genutzt. ${ }^{46}$

$46 \mathrm{Vgl}$. Meissner, R.: Informationstechnologische Lokalzentren als Instrument der Regionalpolitik, in: Raumforschung und Raumordnung, Heft 1-2, 1988, S. 54 ff. 
Ende 1987 existieren in Skandinavien und Finnland bereits 30 ähnliche Projekte, die letztendlich darauf abzielen, eine weitere Entvölkerung dünnbesiedelter Gebiete aufzuhalten. Der Begriff 'Informationstechnologische Lokalzentren' hat sich als oberbegriff in den nordischen Ländern durchgesetzt, wobei zukünftig der dem Norwegischen angelehnte Begriff 'Telematikcenter' synonym verwendet werden soll. Beispielhaft sind im folgenden einige idealisierte Grundzüge dargestellt. "Informationstechnologische Lokalzentren

- beruhen auf der Anwendung moderner Kommunikations- und Informationstechniken;

- wollen die Benutzung entsprechender Anlagen und Geräte einer breiten Bevölkerung zugänglich machen, ohne daß der einzelne zum Kauf der Geräte gezwungen ist;

- erstreben eine allgemeine Hebung des Qualifikationsniveaus der Bevölkerung und des lokalen Wirtschaftslebens (insbesondere in bezug auf die Anwendung von EDV und Kommunikationstechnik);

- wollen neue Arbeitsplätze schaffen, die entweder auf der Befriedigung bisher ungedeckten lokalen Bedarfs oder auf Telearbeit beruhen;

- werden gegründet und betrieben als 'joint venture' durch Zusammenarbeit von Gemeinde und (lokaler) Wirtschaft;

- sind konzipiert als Maßnahme für kleine, periphere Kommunen bzw. Dörfer."47

Gegenüber der Entwicklung in Deutschland, die bedeutende Forschungsaktivitäten auf die Überprüfung der Raumwirksamkeit der Informations- und Kommunikationstechniken verwendet, 48 wird in Norwegen die generelle Möglichkeit einer Vergrößerung des Zentrum-Peripherie-Gefälles konstatiert

47 Ebenda S. 55.

$48 \mathrm{Vgl}$. Georgieff, P. u.a.: Lokale und regionale TelematikVorhaben in der Bundesrepublik Deutschland, a.a.o., s. $11 \mathrm{ff}$. 
und sich auf die Entwicklung von Auffangstrategien konzentriert. 49

Die folgenden Rahmenbedingungen stellen einen Überblick über die wichtigsten Maßnahmen, die von staatlicher seite geschaffen wurden, um die Nutzung der Telematik in peripheren Regionen zu fördern.

Die Digitalisierung des Telekommunikationsnetzes ist in Gang und soll bis 1992 bereits 50\% der Kunden umfassen. Der Ausbau selbst wird nicht lediglich von den Hauptnachfragezentren ausgehend vorgenommen, sondern von vielen über das Land verstreuten digitalisierten 'Inseln'. Hierbei wird den dichter besiedelten Regionen ein gewisser Vorrang vor den dünnbesiedelten gewährt wird.

In Norwegen unterliegt die regionale wirtschaftspolitik 50 dem Kommunal- und Arbeitsministerium. Zusätzlich zu den Investitionshilfen, vergleichbar mit denen der Bundesrepublik Deutschland, werden eine vielzahl von versuchsprojekten in peripheren Regionen finanziell und fachlich unterstützt. Neben der Sammlung von Grundlageninformationen für eine laufende Anpassung des staatlichen Regionalhilfesystems, soll die Diffusion von Innovationen beschleunigt werden. Die projektorientierten Aktivitäten werden in zukunft weiter verstärkt werden, was durch einen Fünfjahresplan zur Entwicklung privater Dienstleistungen in den ländlichen Regionen gewährleistet ist. Ein weiterer schwerpunkt, neben dem Schwerpunkt der Informationstechnologie und neue Dienste, ist die Forschungs-, Entwicklungs- und Beratungs-Tätigkeit, die mit dem Bereich Informationstechnologie stark verflochten ist. Zusätzlich sollen zukünftig die Investi-

49 Was in diesem Fall gleichbedeutend mit der Entwicklung von Strategien zur beschleunigten Anwendung der Telematik in den ländlichen Gebieten ist.

50 Gleich distrikt-sutbygging. 
tionsförderungen stärker als bisher den tertiären sektor berücksichtigen. 51

\subsubsection{Einführung des Télétel-systems in Frankreich}

Ausgehend von der Initiative der Direction Générale France Télécom, eine mit der Deutschen Bundespost Telecom vergleichbare Institution, ist die Einführung des Videotex in Frankreich entscheidend gefördert worden. In den Jahren 1982-83 wurde die generelle Einführung des Dienstes des Elektronischen Telefonbuches beschlossen, was die Entwicklung des Videotex Télétel zur Folge hatte. Mit dieser Entscheidung sollte unter anderem, ausgehend von der studie von Nora und Minc, 52 die Informatisierung der Gesellschaft beschleunigt werden, um die wachsende Verflechtung der Telekommunikation mit der Informatik beherrschen $\mathrm{zu}$ können und nicht von ihr beherrscht $z u$ werden.

Das Télétel-System, das dem Bildschirmtext der Deutschen Bundespost ähnlich ist, wird seit 1982 von der France Télécom angeboten. Entgegen dem Btx, wird beim Télétel dem Telefonkunden ein kostenloses Endgerät namens Minitel, im Austausch gegen das Telefonbuch aus Papier zur Verfügung gestellt und kostenlos gewartet. Das Minitel besteht aus einem kleinen Bildschirm mit einer ausklappbaren Tastatur, das jeder Kunde mit einfachen Handgriffen selbst an sein Telefon anschließen kann und dessen Bedienung keinerlei Training erfordert. 53

$51 \mathrm{Vgl.}$ Meissner, R.: Informationstechnologische Lokalzentren als Instrument der Regionalpolitik, a.a.o., s. 56.

52 Vgl. Nora, S.; Minc, A.: Die Informatisierung der Gesellschaft, a.a.0., s. $13 \mathrm{ff}$.

53 Vgl. o.V.: Btx: Nachhilfe vom Nachbarn, in: Wirtschaftswoche-Special-Supplement, Nr. 3 vom 11.3.1988, S. 28 . 
Mittlerweile sind ca. fünf Millionen Endgeräte von der französischen Post unentgeltlich verteilt worden, wobei lediglich ca. 430.000 Geräte nicht gebührenfrei sind. Diese aufwendigeren Geräte, die primär für den Einsatz in Unternehmen gedacht sind, von denen bisher rund 30 prozent angeschlossen sind, werden gratis abgegeben, kosten jedoch eine monatliche Grundgebühr von ca. 60 Franc. In der Gratisausgabe des Minitels liegt der Schlüssel zum Erfolg dieses Telekommunikationssystems. Gegenüber dem Btx in der Bundesrepublik Deutschland, das gegen Ende 1989 bundesweit mit lediglich 180.000 Endgeräten vertreten ist, hat das Minitel in kürzester zeit die, in der Bundesrepublik noch nicht erreichte, 'Kritische Masse' an Benutzern erreicht. In Frankreich ist das Télétel-system für die wirtschaft lukrativ, mehr als 10.000 Produkte und Dienstleistungen können über das Minitel angewählt werden. Mit dem steigenden Angebot an produkten und Dienstleistungen steigt wiederum die Akzeptanz des Minitels bei den Verbrauchern, was letztendlich das Télétel-System für die wirtschaft zunehmend interessanter macht. 54

Die Einrichtungskosten des Kommunikationssystems und die Produktionskosten für die Endgeräte - ein Minitel kostet ca. 1.000 Franc - liegen in Höhe von sieben Milliarden Franc, die aus vorher gebildeten Rücklagen der France Télécom stammen. 1988 hat die France Télécom mit dem TélételSystem bereits Einnahmen in Höhe von 22,3 Milliarden Franc, von denen 1,3 Milliarden Franc an die Hersteller der über Minitel angebotenen Programme ging. In etwa drei Jahren sollen von den 26 Millionen Telefonanschlüssen sieben bis acht Millionen mit dem Minitel verbunden sein.

54 Vgl. o.V.: Der Minitel erobert in Frankreich die privaten Haushalte, a.a.0., S. 14. 


\subsection{Notwendige Maßnahmen zur Förderung der neuen telekom- munikativen Techniken}

Als Engpaß für die weitere Entwicklung der Telekommunikation wird, wie o.a., nicht die Bereitstellung der Dienste durch die Deutsche Bundespost angesehen, sondern die Innovationen im Bereich der Endgeräte und Anwendungen und die momentan geringe Nutzer-Akzeptanz. Der eigentliche Engpaßfaktor wird primär in der Umsetzung der technischen Möglichkeiten der neuen Telekommunikationsdienste in neue Produkte und entsprechende Produktions- bzw. Bearbeitungsprozesse gesehen. Die Maßnahmen zur Förderung der Anwendung neuer Telekommunikationsdienste leiten sich direkt aus diesen Engpaßfaktoren $a b$ und werden im folgenden dargestellt.

\subsubsection{Allgemeine Maßnahmen zur Akzeptanzsteigerung}

Die Akzeptanz in der Bevölkerung gegenüber den neuen Telekommunikationstechniken stellt eine 'Conditio sine qua non' für die zügige Diffusion der neuen Telekommunikationstechniken dar. Nach den vorliegenden Erkenntnissen, steht der Grad der Akzeptanz in zusammenhang mit den Informationen über die tatsächlichen Vor- und Nachteile der Technik und den unmittelbaren Erfahrungen mit konkreten Anwendungen. 55 Frühzeitige Aufklärung und Einbeziehung sowie entsprechende Aus- und Weiterbildung führt zu einer stärkung der Akzeptanz, wobei grundsätzlich nicht eine Akzeptanz in der Bevölkerung für eine vorgegebene Technik geschaffen werden muß, sondern die Technik sollte für den Menschen akzeptierbar gestaltet und eingesetzt werden.

55 Vgl. Schnöring, T: Wirkungen der Telekommunikationsentwicklung auf Anwender, a.a.0., S. 109. 
Unter dem Aspekt der anhaltenden Arbeitsmarktprobleme und der öffentlich geführten Diskussion über Datenschutz und Datensicherheit ist es notwendig, der öffentlichkeit den positiven Bezug zwischen technischem Fortschritt und wirtschaftlichem Wohlstand zu verdeutlichen. Ausgehend vom Datenschutz und der Datensicherheit, können beispielsweise neue diesbezügliche Qualifikationsmerkmale entwickelt werden, die den Erfolg von neuen Telekommunikationsdiensten steigern. Kubicek beispielsweise fordert, als Vertreter einer Arbeitsgruppe der Gesellschaft für Rechts- und Verwaltungsinformatik, ein Telekommunikationsverkehrsnetz, das für alle Diensteanbieter, Netzbetreiber und Teilnehmer einheitliche klare Regeln vorsieht, wobei Kubicek von der Regelung des Telekommunikationsverkehrs einen positiven Einfluß auf die Nachfrage erwartet. 56

Das Bewußtsein und die sensibilität gegenüber gesellschaftswissenschaftlicher Kritik an modernen Kommunikationssystemen ist bereits, auch innerhalb der Branche, gewachsen. Neben Ingenieursvereinigungen, haben mittlerweile auch die Hersteller die Diskussion mit kritischen Sozialwissenschaftlern aufgenommen. Außer dem ziel der Beseitigung von Mißverständnissen auf beiden seiten, besteht das ziel, berechtigte Kritikpunkte bei der Ausgestaltung der ISDN-Dienstemerkmale $z u$ berücksichtigen. Der Rahmen für die Weiterentwicklung des ISDN wird somit zukünftig nicht nur von den technischen und wirtschaftlichen prämissen bestimmt, sondern auch vom gesellschaftlichen Dialog abhängig sein. 57

56 Vgl. Hoffmann, M.; Lütge, G.: Blind für die Risiken, a.a.0., s. 25 .

57 Vgl. Wagner, P.: Gratwanderung oder Durststrecke, a.a.o.. 


\subsubsection{Anwendungsförderung durch I8DN-8ystem-karketing}

Ausgehend von den Unsicherheiten in der wirtschaft und Verwaltung über die weitere Entwicklung der Informationsund Kommunikationstechniken und ihren Anwendungsmöglichkeiten, erscheint es notwendig, der momentanen Unzufriedenheit uber die segmentierte, interessenbezogene und auf technische Aspekte von Netzen, Diensten und Endgeräten konzentrierte Information, durch ein ISDN-System-Marketing entgegenzuwirken. 58

Ausgehend von einer Untersuchung durch die Unternehmensberatung Arthur D. Little, wurde selbst bei den Postmitarbeitern ein Informationsdefizit bezüglich des ISDN festgestellt. Zusätzlich kursieren in der Fachwelt verballhornende Langformen des ISDN-Kürzels: Innovations subscribers don't need - Neuerungen, die keiner braucht. 59 Ausgehend von den Defiziten in der ISDN-Einführungsstrategie, wird von Arthur D. Little ein konsequenter und umfassender Marketingansatz gefordert, der sich in drei spezifische Punkte untergliedert: 60

1. Internationales Marketingkonzept

2. Systemmarketing

3. Innovationsmarketing

Ein internationales Marketingkonzept für das ISDN ist aufgrund der offenen Kommunikation über nationale Grenzen hinweg notwendig. Durch ein internationales ISDN-Marketingkon-

58 Vgl. Spehl, H.; Messerig-Funk, B.: Chancen und Probleme der wirtschaftlichen Nutzung der Telematik in einer ländlichen Region: Trier, a.a.0., S. 439.

59 Vgl. Hoffmann, M.; Siegele, L.: Kurzschluß bei der Post, a.a.o..

$60 \mathrm{Vgl}$. Knetsch, W.: Herausforderung an das Marketing, in: KoM-Magazin vom Oktober 1988 , S. 27. 
zept, das von der Deutschen Bundespost und den Endgeräteherstellern getragen wird, sollte den relevanten Anwendern die strategische und operative Bedeutung von ISDN, in Bezug auf den existierenden internationalen Kommunikationsbedarf, verdeutlichen.

Von Seiten der Anbieter bestehen, trotz Beginn der Einführung des ISDN, nach wie vor weitreichende Interpretationsdifferenzen bezüglich des ISDN-Verständnisses. Gefordert wird hier ein ISDN-Verständnis, das aus der sicht des Anwenders abgeleitet wird und somit nicht von der ISDN-Lösung alleine, sondern die ISDN-Anwendungen in Zusammenhang mit einer unternehmensweiten Kozeption des Einsatzes von Informations- und Kommunikationstechniken darstellt. Bedingt durch den Integrationsansatz des ISDN, treten gegenüber der reinen Endgeräteentscheidung, organisatorische Einbindungsüberlegungen in den Vordergrund.

Ausqehend vom Innovationscharakter und der Komplexität des Systemanspruchs des ISDN, sind potentielle Anwender im Bereich der kleinen und mittleren Unternehmen nicht in der Lage, die möglichen Vorteile von ISDN gänzlich beurteilen zu können. Ein Innovationsmarketing soll hier aus dem bisweiligen technischen Konzept ein ISDN-Angebot machen, das das Nutzungs- und Einsatzpotential erkennen läßt. Im Mittelpunkt des Innovationsmarketings steht die Auseinandersetzung mit konkreten Anwendungen. 61 Tabelle 4.4 stellt die verschiedenen Phasen des Innovationsmarketings und der verfolgten ziele dar.

$61 \mathrm{Vgl}$. ebenda S. 28 
Tabelle 4.4: Phasen des ISDN-Innovationsmarketing und verfolgte $\mathrm{Ziele} \mathrm{e}^{62}$

\begin{tabular}{|c|c|}
\hline $\begin{array}{c}\text { Phasen des } \\
\text { IS-Innovations- } \\
\text { marketing }\end{array}$ & Phasengerechte Marketing-ziele \\
\hline \hline Know-how-Transfer & $\begin{array}{l}\text { - intern klares IsDN-Verständnis schaffen } \\
\text { (Positionierung, Bedeutung für das eige- } \\
\text { ne Kommunikationsprofil) }\end{array}$ \\
\hline Vorfeld-Marketing & $\begin{array}{l}\text { Auseinandersetzung mit innovationswil- } \\
\text { ligen Kunden } \\
\text { Bestimmung der Marktakzeptanzbedingungen } \\
\text { und Verdeutlichung der Nutzeninnovation }\end{array}$ \\
\hline Pilot-Marketing & $\begin{array}{l}\text { - Zusammenarbeit mit Referenzkunden } \\
\text { Sammlung von praktischen Anwendungser- } \\
\text { fahrungen }\end{array}$ \\
\hline Breiten-Marketing & $\begin{array}{l}\text { Systematische Marktkommunikation auf } \\
\text { der Erenntnisbasis des Vorfeld- und } \\
\text { Pilot-Marketing }\end{array}$ \\
\hline
\end{tabular}

Aus regionalpolitischer sicht bietet sich hier ein Anknüpfungspunkt, im Bereich der gezielten Auseinandersetzung, mit innovationsfreudigen, öffentlichen und privaten organisationen, mit dem ziel, innovative, kommerziell erfolgversprechende ISDN-Anwendungen zu fördern. Ausgehend von Beispielanwendungen, die primär bei Multiplikatoren gefördert werden sollten, werden entscheidende Impulse für die Wahrnehmung der Chancen der wirtschaftlichen Nutzung der Telematik in peripheren Räumen erwartet, die letztendlich zu einer entsprechenden Nachfrage nach den Telekommunikationsdiensten führen. 63

62 In Anlehnung an Knetsch, w.: Herausforderung an das Marketing, a.a.0., s. 28 .

$63 \mathrm{Vgl}$. Lange, S. u.a.: Lokale Initiativen zur Förderung der Anwendung der Telekommunikationstechniken, a.a.0., Speh1, H.; Messerig-Funk, B.: Chancen und Probleme der wirtschaftlichen Nutzung der Telematik in einer ländlichen Region: Trier, a.a.O., S. 418 und Spehl, H.: Entwicklung der Telematik im Trierer Raum - überwiegen die Chancen oder die Probleme?, in: Spehl, H.; MesserigFunk, B. (Hrsg.): Telematik Trier, Trier, 1986, S. 105- 
Weiterhin sollten die Diskrepanzen zwischen den Aussagen der Deutschen Bundespost, daß der Bedarf an Telekommunikationsdiensten, die über das Fernsprechnetz abgewickelt werden können, ohne probleme befriedigt werden kann und den Aussagen der Wirtschaft über schleppende Bedienung, Qualitätsmängel und regionale Defizite in den Informationsaktivitäten, abgebaut und zusätzliche Informationen für interessierte Nutzer bereitgestellt werden. 64

ob sich letztendich die 0.a. Probleme durch eine Anwendungsförderung von einzelnen Beteiligten mit Initialwirkungen lösen lassen, unter dem Aspekt des Informationsaustausches und entsprechend intensivierter Kooperation, oder ob die Information und Beratung in gemeinsam getragenen Institutionen eine bessere Lösung darstellt, muß geprüft werden. Ausgehend von der Vielzahl an ISDN-Projekten, die von der Deutschen Bundespost Telekom im Januar 1990 mit unterschiedlichen Partnern gegründet wurden, 65 erscheint die Abstimmung und Kooperation der einzelnen Projekte, verbunden mit der Weitergabe der Anwendungsinformationen, als ergänzende Maßnahme notwendig.

\subsubsection{Förderung bis zur Mindestteilnehmerzahl}

Bei der Einführung von neuen telekommunikativen Diensten tritt unter anderem ein Problem auf, das in der ökonomischen Theorie unter dem Begriff der kritischen Masse subsummiert wird. Davon ausgehend, daß der Nutzen einer Teilnahme an einem Telekommunikationsdienst mit der Zahl der

117 .

64 Vgl. Spehl, H.; Messerig-Funk, B.: Chancen und Probleme der wirtschaftlichen Nutzung der Telematik in einer ländlichen Region: Trier, a.a.O., S. 439.

$65 \mathrm{Vgl.}$ Cornu, J.: Die Zeit der Zweifel ist vorbei, a.a.o.. 
erreichbaren Teilnehmer wächst, ist die Anzahl der erreichbaren Teilnehmer - die kritische Masse - entscheidend für die Attraktivität eines neuen Dienstes. Eine exakte Bestimmung der Anzahl von Teilnehmern zur Erreichung der kritischen Masse ist sehr schwierig. Letztendlich ist der tatsächlich realisierbare Nutzen, der den Teilnehmern von neuen Diensten erwächst, für die Diffusion entscheidend. Eine Vorhersage über die Diffusion eines neuen Telekommunikationsdienstes, wie beispielsweise des ISDN, ist außerordentlich schwierig, wenn überhaupt möglich. 66

Ausgehend von den startschwierigkeiten der neuen Telekommunikationsdienste, im besonderen des Bildschirmtextes, erscheint eine temporäre Preisreduzierung der Dienste unter die eigentlichen Kosten, als notwendig. Die bis zum erreichen der kritischen Masse befristete Preisreduzierung ist neben den reinen Endgerätekosten auf die Monatsgebühr und auf die Anschlußgebühr ausdehnbar. Die Grundgebühr beim ISDN von 74 DM/Monat wird, beispielsweise besonders bei kleinen und mittleren Unternehmen, als Akzeptanzschwelle angesehen. Ähnlich verhält es sich mit der Anschlußgebühr von 65 DM beim Btx-Dienst. 67

Als Beispiel für eine solche Einführung gilt das französische Minitel, das unter dem Motto 'Die Lampe verschenken und das öl verkaufen' mit großem Erfolg eingeführt wurde und mittlerweile ca. 5 Millionen Anschlüsse hat. Der vergleichbare Btx-Dienst liegt mit ca. 180.000 Anschlüssen erheblich unterhalb der kritischen Masse, die bei etwa 300.000 Geräten liegen könnte, um das gegenseitige Interesse von Informations-Anbietern und -Nutzern in Bewegung $\mathrm{zu}$ bringen. 68

66 Vgl. Schnöring, T: Wirkungen der Telekommunikationsentwicklung auf Anwender, a.a.0., S. 131.

67 Vgl. Sauga, M.: Gezänk über Gebühr, in: Wirtschaftswoche-Special-Supplement $\mathrm{Nr}$. 3/88 vom 11.3.1988, S. 24-32.

$68 \mathrm{Vgl}$. Grab, H.: Bildschirmtext: Masse und Markt, in: 
Eine Förderung bis zum Erreichen der kritischen Masse erscheint besonders unter dem Aspekt der laufenden Kosten für die Deutsche Bundespost als relevant. Das Defizit der Deutschen Bundespost durch den Btx-Dienst für das Jahr 1987 wird auf rund 300 Millionen DM geschätzt. Wird zusätzlich berücksichtigt, daß nach dem Btx-Projektleiter Eric Danke eine Kostendeckung erst bei annähernd einer Millionen Teilnehmer erreicht werden könnte, erscheint eine temporäre Einführungsförderung als wirtschaftliche Notwendigkeit, um nicht erhebliche Einführungsverluste akzeptieren $\mathrm{zu}$ müs$\operatorname{sen} .69$

Diesen Überlegungen zufolge, sind für die zügige Diffusion neuer telekommunikativer Techniken drei Faktoren ausschlaggebend, die letztendlich Ansatzpunkte für eine regionalpolitische steuerung darstellen:

- das Endgeräteangebot und der Endgerätepreis,

- die Telekommunikationsgebühren pro Monat und

- die einmalige Anschlußgebühr.

Wirtschaftswoche-Special-Bürkommunikation Nr. 9 vom 24.2.1989, S. 112 und 121 .

$69 \mathrm{Vgl}$. Schmidt, B.: Bildschirmtext ist immer noch kein Medium für den privaten Markt, a.a.0.. 


\section{Befunde und politische optionen}

Als Ausgangspunkt für die Ableitung politischer Optionen werden im folgenden die Befunde der Untersuchung thesenartig zusammengefaßt:

1. Telekommunikation ist gegenwärtig kein dominierender standortfaktor für Klein- und Mittelbetriebe.

2. Ein homogenes 'Agglomeration $\longleftrightarrow->$ strukturschwache Regionen' Gefälle quantitativer Art zwischen den verschiedenen Regionen und der Adoption neuer telekommunikativer Techniken existiert nicht.

3. Die Charakteristika der standorte haben keinen substantiellen Einfluß auf das qualitative Adoptionsverhalten der Anwender.

4. Zwischen der Größe eines Unternehmens und seinem Adoptionsverhalten existiert ein signifikanter Zusammenhang. Größere Unternehmen sind typische Frühadoptoren, wogegen kleinere Unternehmen $z u$ den Spätadoptoren zählen. 70

5. Einzelbetriebe weisen gegenüber Unternehmen mit mehreren Betriebsstätten eine geringere Adoptionsrate auf.

6. Das Vorhandensein von Forschungs- und Entwicklungsaktivitäten fördert die Adoption neuer Telekommunikationstechniken.

7. Ein homogener Bedarf nach Telekommunikationsdiensten existiert nicht. Der nachgefragte Dienst resultiert

70 Die Investitions- und Betriebskosten der Telekommunikationsdienste haben einen evidenten Einfluß in Bezug auf das temporäre, quantitative und qualitative Adoptionsverhalten. 
primär aus dem Kommunikationsverhalten der jeweiligen Branche. 71

8. Die Nachfrage nach neuen telekommunikativen Diensten wird nicht durch die reine Verfügbarkeit der Dienste geprägt, sondern durch die Kenntnis und den Informationsstand möglicher Anwendungen und Kosten.

9. Mikro- bzw. Personal-Computer-Anwender zählen zu den Frühadoptoren neuer telekommunikativer Techniken.

10. Neugegründete Unternehmen weisen gegenüber länger bestehenden Unternehmen eine höhere Adoptionsrate auf. Dies gilt im besonderen bezüglich der jüngeren Telekommunikationsdienste.

11. Es besteht ein 'Zentrum <-> strukturschwache Regionen' Gefälle bezüglich des Informationsstandes über neue Telekommunikationsdienste, deren Nutzung und deren Kosten.

12. Strukturschwache und ländliche Regionen sind, im Gegensatz $z u$ den Agglomerationen, bereit einen höheren Preis für Telekommunikationsendgeräte $\mathrm{zu}$ bezahlen.

13. Die Einschätzungen gegenüber dem Informations- und Beratungsangebot der Deutschen Bundespost lassen den Schluß zu, daß potentielle Kunden, im besonderen in den strukturschwachen Regionen, mit den existierenden Informations- und Beratungsmaßnahmen nicht erreicht werden.

71 Räumlich unterschiedliche Adoptionsraten lassen sich weitgehend auf unterschiedliche Charakteristiken der jeweils ansässigen Betriebe (Größe, Unternehmenszugehörigkeit, FuE-Tätigkeiten usw.) zurückführen. In Frankfurt ist z.B. der Bereich Unternehmensberatung und in Fulda der Bereich Maschinenbau stärker vertreten. 
14. Die durchschnittlichen Fernmeldekosten von Unternehmen mit peripheren standorten deuten darauf hin, daß die Mehrbelastung, bezogen auf die Gesamtkosten der Unternehmen, lediglich einen geringen Einfluß auf die Wettbewerbsfähigkeit und keinen erheblichen Einfluß auf die standortwahl innerhalb der Bundesrepublik Deutschland hat.

15. Im Bereich der Fernsprechtarife existiert eine starke Kostenüberdeckung bei Ferngesprächen im Inland und demgegenüber eine Kostenunterdeckung bei den Nahbereichsgesprächen.

16. Die technologische Entwicklung im Fernmeldewesen führt zukünftig dazu, daß die 'Dichte' von Verkehrsbeziehungen zunehmend zum dominierenden Kostenfaktor gegenüber der Entfernungskomponente wird.

17. Die räumlichen Wirkungen der Telematik werden zeitlich verzögert erst in der zweiten Hälfte der 90er Jahre spürbar.

18. Die Telekommunikation ist nicht selbst Verursacher oder Auslöser räumlicher Trends, sondern wirkt vor allem als Verstärker vorhandener Trends.

19. Die räumlichen Wirkungen werden eher innerhalb von Verdichtungsräumen, in Form verstärkter suburbanisierung, als zwischen stadt und Land auftreten.

20. Die Angst vor dem 'gläsernen Menschen' kann zu einem wichtigen Adotionshemmis für die neuen telekommunikativen Techniken werden. Datenschutz und Datensicherheit könnten sich zu einem zukünftigen Qualitätsmerkmal herausbilden, das für die weitere Diffusion der neuen Dienste mitentscheidend wird. 
21. Die Akzeptanz gegenüber den neuen telekommunikativen Techniken hängt von der Information über die realen Vor- und Nachteile sowie von der unmittelbaren Erfahrung ab.

22. Im Rahmen der Beschäftigungsfolgen wird eine Polarisierung der Qualifikationsstrukturen erwartet, die sich in einem Abbau der mittlere Qualifikationsebene bei gleichzeitiger Konzentration der Beschäftigten auf hohen und niedrigen Qualifikationsebenen darstellt.

23. Unter dem Aspekt des momentanen Diffusionsstandes, erscheint eine Konzentration der direkten und indirekten Beschäftigungswirkungen auf die günstig strukturierten Verdichtungsräume als möglich, was langfristig eine negative Beschäftigungsentwicklung für die peripheren Regionen zur Folge hätte.

Die Förderung der Telematik entwickelt sich zunehmend zu einem neuen Aufgabenbereich der regionalen Wirtschaftspolitik. Endgültige Antworten über die Effekte der Telematik sowie der Telematikförderung können momentan nicht gegeben werden. Allgemein wird jedoch, bei entsprechend schneller Diffusion, ein günstiger Einfluß auf die interregionale Wettbewerbsfähigkeit prognostiziert. Für die Beurteilung der weiteren Förderungsmaßnahmen sollte zusätzlich bedacht werden, daß ein Warten auf gesicherte wissenschaftliche Erkenntnisse einen Zeitverlust im Wettbewerb darstellt. Darüberhinaus verbergen sich hinter den regionalpolitischen Schlußfolgerungen Ziel- und Interessenskonflikte, die auch zukünftig existieren werden und nicht ohne weiteres auflösbar sind. Diese Konflikte sind teilweise bedingt, durch:

- die Konkurrenz zwischen den Ballungszentren und dem ländlichen Raum,

- die Konkurrenz zwischen den unterschiedlichen Agglomerationen und 
- die interkommunale Konkurrenz innerhalb der Agglomerationen selbst.

Regionale Vorhaben zur Anwendungsentwicklung und -förderung sind zwischenzeitlich bundesweit vertreten. Wichtig erscheint deshalb vor allem ein Informationsaustausch über die bisherigen differenzierten regionalen Entwicklungen, wie beispielsweise in Nordhorn, Karlsruhe und Köln.

Die regionalpolitischen Handlungsempfehlungen umfassen folgende Punkte:

- Eine regionalpolitische Förderung erscheint im besonderen im Bereich der 'institutionellen Infrastruktur', die die Aus- und Weiterbildung, die Anwendungsförderung und die Information und Beratung umfaßt, notwendig. 72 Im besonderen ist hierbei auf die Integration der neuen Telekommunikationstechniken in das existierende Informationsgefüge zu achten, um durch soziale Innovationen die Techniken in den Unternehmen nutzbar zu machen.

- Durch Demonstrationsanlagen oder durch regionale Kommunikationstechnologie-Messen, durch mobile Präsentationsformen, durch verstärkte Beratung im ländlichen Raum und durch eine Informationsbündelung könnten die ungünstigen Transferbedingungen im ländlichen Raum verbessert werden.

- Einer Sicherung der ökonomischen Lebensfähigkeit dient die innovationsorientierte Regionalpolitik, die, über die Schaffung von Kristallisationskernen, einen Anreiz für neue Unternehmen und neue Produktionen geben soll.

- Regionalpolitische Aufmerksamkeit ist im Bereich des

72 Dies kann unter anderem durch eine stärkere Kooperation und einen Ausbau der Beratungs- und Technologietransferstellen in Hessen erfolgen. Eine darüberhinausgehende Koordination mit den Ausbildungszentren ist empfehlenswert. 
ISDN-Ausbaus notwendig. Bei einer weiteren Verzögerung des ISDN-Ausbaus ist eine 'quasi Flächendeckung' mittels Fernanschaltungen möglich, was jedoch bei entsprechender Nachfrage zu Engpässen führen kann. Von Seiten der Regionalpolitik ist darauf $z u$ achten, daß die von der Deutschen Bundespost vorgenommenen Absichtserklärung 'ISDN-Anschlüsse und -Dienste' bis 1993 flächendeckend anzubieten, eingehalten wird.

- Aufgrund der geringen Kostenanteile der Telekommunikationsgebühren an den Gesamtkosten in Unternehmen, kann davon ausgegangen werden, daß diese keinen Einfluß auf die standortwahl haben. Trotzdem sollte bei der Tarifgestaltung auf einen weiteren Abbau der Entfernungsabhängigkeit der Fernsprechgebühren hingewirkt werden, zumindest im Bereich bis $100 \mathrm{~km}$, um eine emotionale Rejektion in den peripheren Gebieten zu vermeiden.

- Unter der Perspektive der Minitel-Erfahrungen in Frankreich, erscheint eine Hinwendung zur Förderung der Einführung neuer Telekommunikationsdienste bis zum Erreichen der kritischen Masse, ab der ein sich selbst tragender Prozeß in Gang gesetzt wird, als erstrebenswert. Dies kann in Form von subventionierten Endgeräten, kostenlosem Anschluß und niedriger Grundgebühr für die Zeit der Einführung erfolgen und würde gleichzeitig die Akzeptanz gegenüber den neuen Telekommunikationstechniken erhöhen.

- Eine entsprechende Einführungs- und Anwendungsförderung sollte auf typische spätanwendergruppen konzentriert werden. Hierzu zählen insbesondere:

- selbständige kleine und mittlere Unternehmen in allen Branchen,

- Betriebe, die noch keine EDV-Anwender sind sowie

- Betriebe ohne Forschungs- und Entwicklungsaktivitäten. 
- Im Rahmen der Anwendungsförderung sollte der ländliche Raum durch einen 'ländlichen Bonus' bei Investitionen im Bereich Telekommunikation zusätzlich gefördert werden.

- Eine verstärkte Förderung von Pilotprojekten, die einer neuartigen Nutzung von Telekommunikationstechniken dienen und die demonstrative schrittmacherdienste für die regionale Wirtschaft erbringen, wird empfohlen.

- Ausgehend von der Verwirklichung des informationellen Selbstbestimmungsrechtes, sollte bereits im Vorfeld der Einführung neuer Telekommunikationsdienste der Einsatz von Instrumenten des technischen Datenschutzes vorgenommen werden, um den Datenschutz für den einzelnen Bürger zu realisieren.

- Durch eine verstärkte Anwendung der Telematik in der öffentlichen Verwaltung kann eine Vorreiter- und Vorbildfunktion übernommen werden. Zusätzlich können die Einführungserfahrungen der örtlichen wirtschaft zur Verfügung gestellt werden, um einen Teil der Anlaufschwierigkeiten in den Unternehmen zu sparen.

- Von seiten der Regionalpolitik sollte verstärkt auf die Deutsche Bundespost eingewirkt werden, im Bereich der erklärungsbedürftigen Telekommunikationsprodukte durch entsprechende Marketingkonzepte eine Diffusionsförderung zu erreichen. Die Konzepte sollten nicht nur branchenorientiert ausgerichtet, sondern mit einem starken Regionalbezug versehen sein.

Aufgabe der regionalen Wirtschaftspolitik ist es, die vermuteten Gestaltungsspielräume auszunutzen und nicht auf die letztendilche Information über positive oder negative Effekte der Telematik zu warten. Die Erfahrungen verschiedener Industriezweige (Büromaschinenindustrie, Uhrenindu- 
strie, Werkzeugmaschinenindustrie) bestätigen zwischenzeitlich die These, daß ein Zögern und verspätetes Umstellen unter dem Druck des Wettbewerbs, letztendlich mehr Arbeitsplätze gefährdet, als ein rechtzeitiges wahrnehmen von Chancen.

Eine Nutzung der Telematik zu Gunsten strukturschwacher Regionen, muß rechtzeitig gefördert werden, wobei mehrere Handlungsalternativen vorliegen. Entwicklungen müssen beobachtet, Defizite ermittelt und auf die Deutsche Bundespost Einfluß genommen werden.

Als vordringlich wird weiterhin die Förderung des Informations- und Erfahrungsaustausches zwischen allen Initiativen angesehen. Parallel dazu sollten ergänzende Forschungsvorhaben zum Kommunikationsverhalten und der Standortentscheidung gefördert werden, die sich im Bereich der organisationsforschung, der Technologiefolgen-Abschätzung und der Telearbeit bewegen.

Die wissenschaftlichen Erfahrungen im Bereich der Technologiefolgen-Abschätzungen haben gezeigt, daß Techniken in der Regel keine drastischen Änderungen auslösen, sondern bereits existierende Trends verstärken. Dies trifft auch auf die Telematik zu.

Grundsätzlich kann die Telematik zur Sicherung und Schaffung von Arbeitsplätzen, zur Unterstützung vorhandener Qualifikation, zur ganzheitlichen Erledigung von Aufgaben und zur Dezentralisierung von Entscheidungsstrukturen verwendet werden, was jedoch letztendlich eine frühzeitige zusammenarbeit aller Beteiligten, als 'Conditio sine qua non' für die Akzeptanz der neuen telekommunikativen Techniken, erfordert. 


\section{Iiteraturverzeichnis}

Aberle, G.: Verkehrspolitik und Regionalentwicklung, Schriftenreihe der Gesellschaft für Regionale strukturentwicklung, Band 6, Bonn 1981

Allen, K.; stevenson, A.: An Introduction to the Italian Economy, London 1974

Allesch, J.: Regionale Entwicklung durch Technologietransfer, Innovation und Qualifikation - das Berliner Modell, in: Frankfurter Allgemeine Zeitung Nr. $131 / 1986$ vom 10 . Juni 1986, Beilage "Berater und Dienstleister", S. B15

Arbeitsgemeinschaft systemanalyse Baden-württemberg: Systemanalytische Untersuchung über Ausgewogenheit, Belastbarkeit und Entwicklungspotential des Landes Baden württemberg und seiner Region Mittlerer Neckar, Stuttgart 1975

Backhaus, K. U.a.: Multivariate Analysemethoden - Eine anwendungsorientierte Einführung, 4. Auflage, Heidelberg u.a. 1987

Bade, F.: Industrieansiedlung in der Bundesrepublik Deutschland - Umfang und regionalpolitische Bedeutung, in: Wochenbericht des Deutschen Instituts für Wirtschaftsforschung, Heft 8,1984, S. 92-98

Bade, F.: Räumliche Anspassungsprozesse, Arbeitsteilung und unternehmerisches Standortverhalten, in: Brugger, E. (Hrsg.): Regionale Innovationsprozesse und Innovationspolitik, Diessenhofen 1984, S. 119-146

Bamberg, G.; Baur, F.: Statistik, München, Wien 1980

Bayerisches staatsministerium für wirtschaft und verkehr: Bayerische Finanzierungshilfen für den gewerblichen Mittelstand, München 1989

Bayerisches statistisches Landesamt (Hrsg.): Veröffentlichungsverzeichnis 1977, München 1977

Beirat für Raumordnung: Instrumente zur steuerung der Entwicklung der Raum- und Siedlungsstruktur, in: Bundesminister für Raumordnung, Bauwesen und städtebau (Hrsg.): Schriftenreihe "Raumordnung", 06.047, 1981, S. $31 \mathrm{ff}$

Bekanntmachung der Richtlinien für die bevorzugte Berücksichtigung von Personen und Unternehmen aus dem Zonenrandgebiet und aus Berlin (West) bei der Vergabe öffentlicher Aufträge vom 11. August 1975 und Berichtigung vom 17.10.1975, in: Bundesanzeiger 1975, Nr. 200 
Bodendorf, F., Lüthi, A.: Neue Informations- und Kommunikationstechnologien, in: Die Unternehmung $\mathrm{Nr} .5,43$. Jahrgang 1989 , S. 347-356

Bölting, H.: Wirkungsanalyse der Instrumente der regionalen Wirtschaftspolitik, Münster 1976

Böltken, F.: Soziale Akzeptanz neuer Techniken im regionalen Vergleich, in: Informationen zur Raumentwicklung, Heft 4,1989 , S. 245-258

Bördlein, R.: Informationstechnisch bedingte raumstrukturelle Veränderungen im Frankfurter Umland, untersucht am Beispiel der Anbieter neuer Informations- und Kommunikationstechnologien, Frankfurt 1986

Böventer, von E.: Die räumlichen Wirkungen von öffentlichen und privaten Investitionen, in: Arndt, H.; Swatek, D. (Hrsg.): Grundfragen der Infrastrukturplanung, Berlin 1971, S. 167-200

Brepohl, K.: Telematik - Die Grundlage der Zukunft, Bergisch Gladbach 1982

Brösse, U.: Ziele in der Regionalpolitik und in der Raumordnungspolitik, Berlin, New York 1972

Brösse, U.: Raumordnungspolitik als integrierte Entwicklungspolitik - Kritische Analyse, Zielsystem, Reformansätze, Schriften der Kommission für Wirtschaftliche und Sozialen Wandel, Band 97, Göttingen 1975, S. 222 ff

Brösse, U.: Raumordnungspolitik, Berlin, New York 1982

Brohm, W.: Stadtentwicklungsplanung und neues Bodenrecht, in: Die Verwaltung, Heft 4, 1976, S. $405 \mathrm{ff}$

Brugger, E.: Innovationsorientierte Regionalpolitik, in: Geographische Zeitschrift, Heft 3, 1980, S. 173-198

Buchner, W.: Neuorientierung der regionalen strukturpolitik, in: Raumforschung und Raumordnung, Heft 4, 1985, S. $168-172$

Buhr, W.: Die Abhängigkeit der räumlichen Entwicklung von der Infrastrukturausstattung, in: Grundfragen der Infrastrukturplanung für wachsende Wirtschaften, Schriften des Vereins für Socialpolitik, Band 58, Berlin 1971, S. $106 \mathrm{ff}$

Bullinger, D.: Räumliche Auswirkungen neuer Informationsund Kommunikationstechnologien, Diskussionspapier zur Herbstsitzung der DGS-Sektion stadt- und Regionalsoziologie, 17.-20.11.1983, Basel 1983 
Bullinger, D.: Tendenzen betrieblichen standortwechsels in Ballungsräumen, in: Raumforschung und Raumordnung, Heft 3 , 1983 , S. $82-89$

Bundesbaugesetz in der Fassung der Bekanntmachung vom 18 . August 1976, in: BGBl. I, S. 2257, ber. BGBl. I, S. 3617, geändert durch Art. $9 \mathrm{Nr} .1$ Vereinfachungsnovelle $\mathrm{v}$. 03.12.1976, in: BGBl. I, S. 3281, \$ 1.3 .

Bundesforschungsanstalt für Landeskunde (Hrsg.): Informationen zur Raumentwicklung, Themenheft "Arbeitslosenquote als Indikator der Regionalpolitik?", Heft 3/4, 1980

Bundesministerium für das Post- und Fermeldewesen: Mittelfristiges Programm für den Ausbau der technischen Kommunikationssysteme, Bonn o.J.

Bundesminister für das Post- und Fernmeldewesen: ISDN - die Antwort der Deutschen Bundespost auf die Anforderungen der Telekommunikation von morgen, Bonn 1984

Bundesministerium für das Post- und Fernmeldewesen: Konzept der Deutschen Bundespost zur Weiterentwicklung der Fernmeldeinfrastruktur, Bonn 1984

Bundesministerium für das post- und Fermeldewesen (Hrsg.): Das ISDN-Angebot der Deutschen Bundespost - Ein örtlicher und zeitlicher Überblick, Bonn 1988

Bundesminister für Forschung und Technik: Informationstechnik: Konzeption der Bundesregierung zur Förderung der Entwicklung der Mikroelektronik, der Informations- und Kommunikationstechniken, Bonn 1984

Bundesminister für Forschung und Technik: Bundesbericht Forschung 1988, Bonn 1988

Bundesministerium für Wirtschaft (Hrsg.): Wirksame Regionalpolitik - Fortschritte in den Regionen, Bonn 1980

Bundesministerium für Wirtschaft (Hrsg.): Regionale Wirtschaftsförderung in der Bundesrepublik Deutschland, Bonn, Duisdorf 1989

Bundesminister für wirtschaft: Richtlinien des Bundesministers für Wirtschaft über die Förderung von Unternehmensberatungen für kleine und mittlere Unternehmen vom 20 . November 1989, Bonn 1989

Bundesminister für wirtschaft (Hrsg.): Der Mittelstand Förderungsmaßnahmen des Bundes für mittelständische Unternehmen, Freie Berufe und Existenzgründer, Bonn 1989

Bundesminister für Wirtschaft: Bekanntmachung des Bundesministers für Wirtschaft vom 2.1.1990, in: Bundesanzeiger Nr. 15 vom 23.1.1990 
Buttler, F. u.a.: Grundlagen der Regionalökonomie, Reinbeck 1377

Cornu, J.: Die Zeit der Zweifel ist vorbei, strategien für den Durchbruch von ISDN, in: Frankfurter Allgemeine Zeitung Nr. 67 vom 20. März 1990, S. B2

Der Bundeminister für Forschung und Technologie und der Bundesminister für wirtschaft (Hrsg.): Zukunftskonzept Informationstechnik, Bonn 1989

Der Hessische Minister für Wirtschaft und Technik: Nordhessen - Strukturpolitischer Bericht der Hessischen Landesregierung, Wiesbaden 1986

Der Hessische Minister für Wirtschaft und Technik: Jahresbericht 1986, Frankfurt 1987

Deutsche Bundespost: ISDN - die Antwort der Deutschen Bundespost auf die Anforderungen der Telekommunikation von morgen, Bonn 1984

Deutsche Bundespost: Die wichtigsten Gebühren, 0.0., stand September 1988

Deutsche Bundespost: Mit Videokommunikation in die zukunft blicken, in: Videokommunikation visuell 1, 1988, S. 2

Deutsche Bundespost: MEDKOM ein Anwendungsprojekt zur Erprobung der medizinischen Kommunikation im Vorläufer-Breitband-Netz VBN, ohne ort o.J.

Deutsche Bundespost: Videokonferenz/Videokommunikation Entgelte -, 0.0., Stand 1989

Deutsche Bundespost: Dienste der Deutschen Bundespost für die Textkommunikation, 0.0. April 1989

Deutsche Bundespost: Telebox - Das Angebot der Deutschen Bundespost für Mitteilungen von Person zu Person, 0.0. Januar 1989

Deutsche Bundespost: Post-Seminare 1989, Frankfurt 1989

Deutscher Bundestag: Grundsätze der regionalen Wirtschaftspolitik, Bundestagsdrucksache V/2469, Bonn 1968

Deutscher Bundestag: Raumordnungsprogramm für die großräumige Entwicklung des Bundesgebietes (Bundesraumordnungsprogramm), Bundestagsdrucksache 7/3584, Bonn 1975

Deutscher Bundestag: Raumordnungsbericht 1978 und Materialien, in: Schriftenreihe "Raumordnung" des Bundesminister für Raumordnung, Bauwesen und Städtebau, Heft 06.040, Bonn 1979, Anlage 1 
Deutscher Bundestag: Programmatische Schwerpunkte der Raumordnung, Bundestagsdrucksache 10/3146, Bonn 1985

Deutscher Bundestag: Vierzehnter Rahmenplan der Gemeinschaftsaufgabe "Verbesserung der regionalen Wirtschaftsstruktur", Bundestagsdrucksache 10/3562, Bonn 1985

Deutscher Bundestag: Raumordnungsbericht 1986, Bundestagsdrucksache 10/6072, Bonn 1986

Deutscher Bundestag: Siebzehnter Rahmenplan der Gemeinschaftsaufgabe "Verbesserung der regionalen Wirtschaftsstruktur", Bundestagsdrucksache 11/2362, Bonn 1988

Dick, A.: Übergang zur flexiblen Planung, in: Bayerischer Staatsanzeiger $\mathrm{Nr} .25$ vom 23.6.1978

Dienel, P.: Die Planungszelle. Der Bürger plant seine Umwelt. Eine Alternative zur Establishment- Demokratie, Opladen 1978

Dupke, S., Ganser, K.: Behördenstandorte als Instrument der Raumordnung, in: Informationen zur Raumentwicklung, Heft 1, 1975 , S. $1-42$

Eberstein, H.: Grundlagen der Regionalpolitik und ihre wesentlichen Grundsätze, in: Handbuch der regionalen Wirtschaftsförderung, Köln ab 1971, Abschnitt A III, S. 15

Eckey, H.-F.: Regionale Engpaßfaktoren, in: Nordrhein-Westfalen in der Krise - Krise in Nordrhein-Westfalen? Schriften des Rheinisch-Westfälischen Instituts für wirtschaftsforschung Essen, Heft 46, Berlin 1985, S. 7-37

Eckey, H.-F. u.a.: Sozioökonomische und ökologische Bedingungen für die Entwicklung Nordhessens, Gesamthochschule Kassel, Kassel 1987

Egner, E.: Raumwirtschaftspolitik, in: Handwörterbuch der Sozialwissenschaften, 8. Band, Göttingen, Tübingen 1964, S. $694-794$

Engelhardt, W.: Betriebliche Absatz- und Beschaffungspolitik, Bochum 1975

Enquete-Kommission: Neue Informations- und Kommunikationstechniken, Kommission des Deutschen Bundestages, Drucksache $9 / 2442$, Bonn 1983

Ernst-zinkhahn-Bielenberg: Bundesbaugesetz, Kommentar, Fortlaufende Lieferungen

Ewe, J.: Otello soll es richten, in: Frankfurter Allgemeine Zeitung Nr. 67 vom 20. März 1990, S. B5 
Ewers, H.: Räumliche Innovationsdisparitäten und räumliche Diffusion neuer Technologien, in: Brugger, E. (Hrsg.): Regionale Innovationsprozesse und Innovationspolitik, Diessenhofen 1984 , S. 97-118

Ewers, H.; Fritsch, M.: Telematik und Raumentwicklung, Bonn 1985

Ewers, H.; Fritsch, M.: Die räumliche Verbreitung von computergestützten Techniken in der Bundesrepublik Deutschland, in: Böventer, von E. (Hrsg.): Regionale Beschäftigung und Technologieentwicklung, Berlin 1989, S. 81-114

Ewers, H.-J.; Wettmann, R.: Innovationsorientierte Regionalpolitik, Bonn 1980

Ewers, H.; Wettmann, R.: Innovationsorientierte Regionalpolitik - Überlegungen zur einem regionalstrukturellem Politik- und Forschungsprogramm, in: Informationen zur Raumentwicklung, 7, 1978, S. 467 ff

Ewringmann, D. u. a.: Die Gemeinschaftsaufgabe "Verbesserung der regionalen Wirtschaftsstruktur" unter veränderten Rahmenbedingungen, Berlin 1986

Faller, P.: Die Entfernungsstaffeln der Deutschen Bundesbahn unter raumordnerischen Gesichtspunkten, in: Jochimsen, R. u.a. (Hrsg.): Theorie und Praxis der Infrastruktur, Schriften des Vereins für Socialpolitik, Band 54, Berlin 1970

Fiedler, H.; Wodtke, K.: Technologiezentren in der Bundesrepublik Deutschland 1988, Berlin 1988

Fischer, K.: Telekommunikation Raumordnung und regionale Strukturpolitik, Köln 1984

Fleckenstein, K.: Aufgabenteilung in der regionalen Wirtschaftsförderung, in: Raumforschung und Raumordnung, Heft 4, 1985, S. 173-180

Fölber, R.: Bedarfsuntersuchung für die Fernmeldenetze in Rheinland-Pfalz, in: Deutsche Telepost Consulting GmbH (Hrsg.): Aspekte der Telekommunikationsentwicklung im Land Rheinland-Pfalz, Bonn, Mainz 1987

Friedrich, J. u.a.: Organisationsmodelle für das Büro von morgen, in: Office Management 3/I-1987, S. 16-22

Fritsch, M.: Räumliche Unterschiede der Telematik-Adoption in Industriebetrieben der Bundesrepublik Deutschland, in: Akademie für Raumforschung und Landesplanung (Hrsg.): Räumliche Wirkungen der Telematik, Forschungs und sitzungsberichte Band 169, Hannover 1987, S. 301-336 
Fritsch, M.; Ewers, H.: Telematik und Raumentwicklung, Bonn 1985

Fröhlich, R.: Von Angesicht zu Angesicht - Bildtelefon geht Ende des Jahres in Betrieb, in: Frankfurter Allgemeine Zeitung Nr. 67 vom 20. März 1990, S. B5

Fürst, D. u.a.: Standortwahl industrieller Unternehmen, Bonn 1973

Fürst, D. u.a.: Regionale Wirtschaftspolitik, Tübingen, Düsseldorf 1976

Funck, R.: Instrumente der Regionalpolitik, in: Schneider, H. (Hrsg.): Beiträge zur Regionalpolitik, Schriften des Vereins für Socialpolitik, Band 41, Berlin 1968, S. 111-125

Funck, R.: Technologiepolitik und räumliche struktur, in: Böventer, von E. (Hrsg.): Regionale Beschäftigung und Technologieentwicklung, Berlin 1989

Funke + Huster Elektrizitätsgesellschaft mbH co. KG (Hrsg.): Der neue Temex-Service der Bundespost, in: Frankfurter Allgemeine Zeitung $\mathrm{Nr} .7$ vom 9. Januar 1990

Garstka, H.: Schutz von Persönlichkeitsrechten bei der Nutzung neuer Medien, in: Kilian, W. (Hrsg.): Neue Medien für die Individualkommunikation, München 1984, S. 79-85

Gee, C. u.a.: Infrastrukturelle und wirtschaftsstrukturelle Bestimmungsgründe der industriellen standortwahl, stuttgart 1980

Genosko, J.: Die innovationsorientierte Regionalpolitik, Eine wirksame Handlungsalternative?, in: Raumforschung und Raumordnung, Heft $2 / 3,1986$, S. 107-115

Georgieff, P. u.a.: Lokale und regionale Telematik-Vorhaben in der Bundesrepublik Deutschland, Fraunhofer-Institut für Systemtechnik und Innovationsforschung (Hrsg.), Karlsruhe 1987

Gesetz über die Gemeinschaftsaufgabe "Verbesserung der regionalen Wirtschaftsstruktur" vom 6. Oktober 1969, BGBl. I, S. 1861

Gesetz zur Förderung des zonenrandgebiets (Zonenrandförderungsgesetz) vom 5. August 1971, in: BGB1. I, S. 1237

Giersch, H.: Aufgaben der Strukturpolitik, in: Hamburger Jahrbuch für Wirtschafts- und Gesellschaftspolitik, 9. Jahrgang, Hamburg 1964

Goedecke, O.: Regionalplanung in Bayern, DIFU-Seminar Regionale Entwicklungsplanung, Berlin 1976 
Grab, H.: Bildschirmtext: Masse und Markt, in: Wirtschaftswoche-Special-Bürkommunikation, $\mathrm{Nr}$. 9 vom 24.2.1989, S. 112 und 121

Grabow, B.; Henckel, D.: Räumliche Verteilung von Unternehmen der Informationstechnologie, in: Akademie für Raumforschung und Landesplanung (Hrsg.): Räumliche Wirkungen der Telematik, Hannover 1987, S. 245-272

Gräf, P.: Analyse räumlicher Verteilungsmuster von Anwendern in der Bundesrepublik Deutschland, in: Akademie für Raumforschung und Landesplanung (Hrsg.): Räumliche Wirkungen der Telematik, Hannover 1987, S. 337-366

Green, P., Tull, D.: Methoden und Techniken der Marketingforschung, 4. Auflage, Stuttgart 1982

Grundgesetz: Grundgesetz mit Grundvertrag, Menschenrechtskonvention, Bundeswahlgesetz, BVerfassGerichtG, Parteiengesetz, Beck Verlag (Hrsg.), 20. Auflage, München 1980

Gschwind, F., Henckel, D.: Innovationszyklen der Industrie - Lebenszyklen der städte, in: Bauwelt 24, Stadtbauwelt $82 / 1984$, S. $134-136$

Gundrum, U.: Regionale Telematikanwendungen in der Bundesrepublik Deutschland, in: Der Städtetag 11, 1987, S. 677682

Haist, W.: Weiterentwicklung der Fermeldesysteme, in: Elias, D. (Hrsg.): Telekommuniaktion in der Bundesrepublik Deutschland 1982, Heidelberg 1982, S. 155-160

Haldenwang, H.: Anwendungen neuer Informations- und Kommunikationstechniken in Ostbayern, in: Spehl, H.; MesserigFunk, B. (Hrsg.): Telematik Trier, Ergebnis eines Symposiums, Beiträge aus Forschung und Praxis, Band I, Trier 1986 , S. 62-90.

Hall, P.: The Geography of the Fifth Kondratieff Cycle, in: New Society, Band 55, 1981, S. 535-537

Hamm, W.: Milliardenstrom in die Ballungsgebiete, in: Frankfurter Allgemeine Zeitung Nr. 169 vom 25. Juli 1989, S. 9

Hartke, S.: Selbstverantwortete regionale Entwicklung im Rahmen der Raumordnung, in: Raumordnung und Raumforschung, Heft 2, 1984

Hartung, H.: Statistik: Lehr- und Handbuch der angewandten Statistik, 6. Auflage, München, Wien, Oldenbourg 1987

Heinze, G., Kill, H.: Chancen und Grenzen der neuen Informations- und Kommunikationstechniken - zur übertragung verkehrsevolutorischer Erfahrugen auf die Telekommunika- 
tion, in: Akademie für Raumforschung und Landesplanung (Hrsg.): Räumliche Wirkungen der Telematik, Hannover 1987, S. $21-72$

Heller, K.; Rosemann, B.: Planung und Auswertung empirischer Untersuchungen, 2. Auflage, Stuttgart 1981

Henckel, D. u. a.: Informationstechnologie und stadtentwicklung, Stuttgart 1984

Hessischer Minister für Wirtschaft und Technik: Nutzung der Informations- und Kommunikationstechnik in Hessen, Beiträge zur Wirtschaftspolitik in Hessen, Heft 13, Wiesbaden 1987

Hessiches Ministerium für wirtschaft und Technik: Strukturpolitischer Bericht für Nord- und Osthessen, Beiträge zur Wirtschaftspolitik in Hessen, Heft 15, Wiesbaden 1988

Hessisches Ministerium für Wirtschaft und Technik: Strukturpolitischer Bericht für Mittelhessen - Beiträge zur Wirtschaftspolitik in Hessen, Heft 16, Wiesbaden 1988

Hessisches Ministerium für Wirtschaft und Technik: Jahresbericht 1988, Wiesbaden 1989

Hoberg, R.: Raumwirksamkeit neuer Kommunikationstechniken Innovations- und diffusionsorientierte Untersuchung, in: Vorstand der Gesellschaft für Regionalforschung e.V. (Hrsg.): Jahrbuch für Regionalwissenschaft, Göttingen 1983, S. $5-38$

Hoberg, R.: Zur Analyse von räumlichen Diffusionsprozessen bei der Telematikanwendung, in: Akademie für Raumforschung und Landesplanung (Hrsg.): Räumliche Wirkungen der Telematik, Hannover 1987, S. 73-90

Hoffmann, M.; Lütge, G.: Blind für die Risiken, in: Die Zeit Nr. 6 vom 3. Februar 1989, S. 24

Hoffmann, M.; siegele, L.: Kurzschluß bei der Post, in: Die Zeit $\mathrm{Nr} .5$ vom 27 . Januar 1989, S. 37

Holtz, W.: Öffentliche Auftragsvergebung, in: Handwörterbuch der Sozialwissenschaften, 1. Band, Stuttgart 1956, S. 433-437

Hopfinger, H.: Erfolgskontrolle regionaler Wirtschaftsförderung, Erlangen 1982

Hopfinger, H.: Für eine gespaltene Regionalpolitik unter veränderten Rahmenbedingungen, in: Raumforschung und Raumordnung, Heft 3, 1983, S. 90-100

Hübler, K.-H.: Rechtsgrundlagen und Programme der Raumordnung, in: Eberstein, H. (Hrsg.): Handbuch der regionalen wirtschaftsförderung, Abschnitt 1 IV A, Köln 1971, S. 1-19 
Innovations- und Technologie-Beratungsstelle Hessen: Technologieberatung - Hilfe bei Forschung und Entwicklung in Hessen, Frankfurt o.J.

Innovations- und Technologie-Beratungsstelle der hessischen Industrie- und Handelskammern: Informationen aus Datenbanken - Beschaffung und Beratung, Frankfurt 1989

Jochimsen, R.: Theorie der Infrastruktur, Tübingen 1966

Jochimsen, R.; Gustafsson, K.: Infrastruktur. Grundlage der marktwirtschaftlichen Entwicklung, in: Simonis, U. (Hrsg.): Infrastruktur - Theorie und Politik, Neue wissenschaftliche Bibliothek. Wirtschaftswissenschaften, Band 88, Köln 1977

Julitz, L.: Bayern, Baden-württemberg und Hessen liegen vorn, in: Frankfurter Allgemeine Zeitung Nr. 254 vom 31. Oktober 1988, S. 18

Junkernheinrich, M.: Dezentralisierung der Wirtschaftspolitik, in: Wirtschaftsdienst XI, 1985, S. $576 \mathrm{ff}$

Raiser, W.: Entwicklungslinien der Telekommunikation, in: Konrad Adenauer Stiftung (Hrsg.): Neue Medien, Melle 1984, S. $67-74$

Rieser, A.; Kubicek, H.: Organisation, 2. Aufl., Berlin/New York 1983

Rlaus-stöhner, U.; Zabel, J.: Möglichkeiten und Anwendungsbarrieren für den Einsatz neuer Informations und Kommunikationstechniken in einer peripheren Region: Fallstudie Waldeck-Frankenberg, in: Akademie für Raumforschung und Landesplanung (Hrsg.): Räumliche wirkungen der Telematik, Hannover 1987 , S. 393-416

Rlatzky, S.: Automation, Betriebsgröße und die Verteilung von Entscheidungskompetenzen - Der Kaskadeneffekt, in: Grochla, E. (Hrsg.): Organisationstheorie, 2. Bd., stuttgart 1976 , S. 518-529

Rlemmer, P.: Zur Begründung einer regionalen Wirtschaftspolitik im Rahmen der Marktwirtschaft, in: das wirtschaftsstudium (wisu), 6. Jahrgang, 1977

Knetsch, W.: Herausforderung an das Marketing, in: KOM-Magazin vom Oktober 1988, S. 27-28

König, K.; schimanke, D.: Räumliche Planungen im politischadministrativen System der Länder, Abhandlungen der Akademie für Raumforschung und Landesplanung, Band 81, Hannover 1980

Rordey, N.: Raumstrukturelle wirkungen neuer Informationsund Kommunikationstechnologien, dargestellt anhand der 
Strategien öffentlicher Verwaltung und unternehmerischer Standortentscheidungen, Frankfurt 1986

Kordey, N.: Gebührenpolitik im Fernmeldewesen und Raumordnung, in: Raumforschung und Raumordnung, Heft 5-6, 1988, S. 215-225

Rreditanstalt für Wiederaufbau: Zusammenstellung der Kreditprogramme der Kreditanstalt für Wiederaufbau für Unternehmen der gewerblichen wirtschaft, Frankfurt 1990

Krummacher, M., Wienemann, M.: Neue Technologien und Hoffnung auf eine Renaissance für das Ruhrgebiet, in: Raumplanung $32 / 1986$, S. 37-40

Rüpper, U.: Neue Technologien, Wirtschaftswachstum und Innenstadtentwicklung, in: Archiv für Kommunalwissenschaften 24,1985

Küpper, U.: Aktive lokale standortenwicklung im strukturwandel, Initiativen in Köln 1983-1988, in: Informationen zur Raumentwicklung, Heft 4, 1989, S. 269-276

Kubicek, H.; Mettler-Meibom, B.: Alternative Entwicklungspfade der Telekommunikationspolitik, B46-47, S. 35

Läpple, D.: Neue Technologien in räumlicher Perspektive, in: Informationen zur Raumentwicklung, Heft 4, 1989, S. 213-226

Landesamt für Datenverarbeitung und statistik Nordrheinwestfalen (Hrsg.): Veröffentlichungen 1977, Düsseldorf 1977

Landesentwicklungsbericht Baden-Württemberg 1975. Band 1

Landesentwicklungsplan VI: "Festlegung von Gebieten für flächenintensive Großvorhaben (einschließlich standorte für die Energieerzeugung), die für die Wirtschaftsstruktur des Landes von besonderer Bedeutung sind" des Landes NordrheinWestfalen vom 8.11.1978

Lange, S.: Telematik und regionale Wirtschaftspolitik, Köln 1985

Lange, S.: Teleports - Entstehung und Förderung der Telematik, in: Innovation und Management, Heft 3, 1986, S. 285298

Lange, S. U.a.: Lokale Initiativen zur Förderung der Anwendungen der Telekommunikation, Köln 1986

Lange, S. U.a.: Lokale Initiativen zur Förderung der Anwendung der Telekommunikationstechniken, Telematik und regionale Wirtschaftspolitik, Band II, Köln 1986 
Lange, S.: Neue Aufgaben für die kommunale wirtschaftspolitik, in: Akademie für Raumforschung und Landesplanung (Hrsg.): Räumliche wirkungen der Telematik, Hannover 1987, S. 503-519

Lange, S.: Teleports: Neue Aufgaben für die kommunale Wirtschaftspolitik, in: ONLINE'87, 10. Europäische Kongreßmesse für Technische Kommunikation, Hamburg 1987, S. 17.1.0117.1 .16

Lange, S.: Lokales Potential für den Weltmarkt, in: net, 42 Heft 9, 1988, S. 334-338

Langen, B., Biemen, A.: ISDN und Spezialnetze. Wo liegen die voraussichtlichen Nutzungsschwerpunkte des ISDN innerhalb eines Datenübermittlungssystems?, in: office Management, Heft 6, 1988, S, 14-20

Langenbucher, W.: Individuum und Haushalt als Informationssucher - Chancen für Bildschirmtext, in: Witte, E. (Hrsg.): Telekommunikation für den Menschen, Berlin 1980, S. 118-139

Lauschmann, E.: Grundlagen eienr Theorie der Regionalpolitik, Hannover 1976

Lenk, T.: Telearbeit: Möglichkeiten und Grenzen einer telekommunikativen Dezentralisierung von betrieblichen Arbeitsplätzen, Berlin 1989

Leontief, W.; Duchin, F.: The Future of Automaiton on Workers, Cambridge 1986

Lydorf, H.: TEMEX - ein neuer Fernmeldedienst der Deutschen Bundespost, in: Bundesminister Schwarz-Schilling, Florian, W. (Hrsg.): Jahrbuch der Deutschen Bundespost 1986, Bad Windsheim 1986, S. 237-280

Martin, H.: Die technische Entwicklung zum ISDN, in: Telematikmagazin, 7. Jahrgang, 1, 1987, S. 30-34

Matzner, E. U.a.: Beschäftigungsrisiko Innovation? - Arbeitsmarktwirkungen moderner Technologien; Befunde aus der Meta-Studie, Berlin 1988

Meissner, R.: Informationstechnologische Lokalzentren als Instrument der Regionalpolitik, in: Raumforschung und Raumordnung, Heft 1-2, 1988, S. 54-62

Menges, G.: Die Statistik - Zwölf Stationen des statistischen Arbeitens, Wiesbaden 1982

Meyer-Rrahmer, F.: Innovation Behaviour and Regional Indigenous Potential, in: Regional studies, Vol. 19, Heft 6, 1985 , S. 523-535 
Miller, A.: "Computer and Privacy", in: Hoffmann, W.; Mills Moore, J. (Eds.): Ethics and the Managment of Computer Technology. Proceedings of the Fourth National Conference on Business Ethics, Cambridge, Massachusetts 1982, S. 93114

Ministerium für Wirtschaft, Mittelstand und Technologie des Landes Baden-württemberg (Hrsg.): Wirtschaftsförderungsprogramm - Existenzgründung - Existenzfestigung - Technolgieorientierte Unternehmensgründung, Stuttgart 1987

Ministerium für wirtschaft, Mittelstand und Technologie des Landes Baden-württemberg (Hrsg.): Wirtschaftsförderungsprogramm - Technologieförderung, stuttgart 1989

Minister für wirtschaft, Mittelstand und Technologie des Landes Nordfhein-Westfalen: NRW Technologieprogramm Wirtschaft und Programm Zukunftstechnologien, Düsseldorf 1985

Minister für wirtschaft, Mittelstand und Technologie des Landes Nordfhein-Westfalen: Wirschaftspolitik für Nordrhein-Westfalen -Zwischenbilanz des MWMT-, Düsseldorf 1987

Minister für wirtschaft, Mittelstand und Technologie des Landes Nordrhein-Westfalen und Deutsche Bundespost (Hrsg.): Telekommunikations-Atlas Nordrhein-Westfalen Band 2, 0.0 . September 1988

Minister für wirtschaft, Mittelstand und Technologie des Landes Nordfhein-Westfalen: Telekommunikations-Produkte und -Dienstleistungen, Chancen für Hersteller und Anbieter in NRW, Düsseldorf 1988

Minister für Wirtschaft, Mittelstand und Technologie des Landes Nordrhein-Westfalen (Hrsg.): Zukunftswerkstatt Nordrhein-Westfalen, Düsseldorf 1989

Mock, W.: Stützen des Strukturwandels, Technologiezentren: Ein wichtiger Faktor regionaler Wirtschaftspolitik, in: VDI Nachrichten Nr. 16/21. April 1989, S. 22

Müdespacher, A.: Informationstechnologie, räumliche Diffusionsprozesse und Adoptionsverhalten der schweizerischen Wirtschaft, TH Zürich, Institut für Orts-, Regional- und Landesplanung (Hrsg.), Zürich 1986

Muiller, H.: Methoden zur regionalen Analyse und Prognose, in: Akademie für Raumforschung und Landesplanung (Hrsg.): Taschenbücher zur Raumplanung, Band 1, Hannover 1973

Müller, K.: Arbeitsmarktorientierte Regionalpolitik, Nr. 86 der Arbeitsmaterialien der Akademie für Raumforschung und Landesplanung, Hannover 1984 
Naylor; H., Türke, K.: Welche Wirkungen können neue Kommunikationsmedien auf Raumordnung und stadtentwicklung haben?, in: Informationen zur Raumentwicklung, Heft 3, 1982 , S. $175-186$

Neumann, K., schnöring, T.: Das ISDN - Ein Problemfeld aus volkswirtschaftlicher und gesellschaftspolitischer sicht, in: Jahrbuch der Deutschen Bundespost 1986, Bad Windsheim 1986 , S. 551-557

Niedersächsisches Ministerium für Wirtschaft, Technologie und Verkehr: Förderung von Forschungs- und Entwicklungsvorhaben auf dem Gebiet der Produkt- und Verfahrensinnovation, Hannover 1989

Niemeier, H. U.a.: Landesplanungsrecht Nordrhein-Westfalen, Essen 1977

Nieschlag, R. U.a.: Ein entscheidungstheoretischer Ansatz, Berlin 1971

Nora, S.; Minc, A.: Die Informatisierung der Gesellschaft, Frankfurt, New York 1978

Nuppnau, H.: Wirkungen der Zonenrandförderung, Hamburg 1974

Oberpostdirektion Frankfurt: Leitfaden Telekommunikation, Band 1, Frankfurt 1986

Oberpostdirektion Frankfurt: Leitfaden Telekommunikation, Ergänzung zu Band 2, Frankfurt 1986

Oberpostdirektion Frankfurt (Hrsg.): ISDN-Einführung in Hessen, Frankfurt 1988

d'Orville, H.: Probleme einer Erfolgskontrolle regionalpolitischer Maßnahmen, Frankfurt, Bern, Las Vegas 1979

o.v.: Regionalsteuerung für Gastarbeiter nicht mehr zweckmäßig, in: Staatsanzeiger für Baden-württemberg Nr. 26, 1977

o.v.: Endogene Entwicklungsstrategien, in: Informationen zur Raumentwicklung, Heft $1 / 2,1984$

o.v.: Anforderungen an die Raumordnungspolitik in der Bundesrepublik Deutschland, in: Raumforschung und Raumordnung, Heft 2/3, 44. Jahrgang, Köln 1986, S. 57-60

o.v.: Btx: Nachhilfe vom Nachbarn, in: WirtschaftswocheSpecial-Supplement Nr. 3 vom 11.3.1988, S. 28-29

o.v.: Künftige ISDN-Anwender müssen überzeugt werden, in: VDI-Nachrichten vom 11.3 .1988 
0.V.: Glasfaser unter der Lupe - Berkom untersucht Breitbandkommunikation, in: VDI-Nachrichten vom 18.11.1988

o.v.: Steuern + Standort, in: wirtschaftswoche $\mathrm{Nr} .42$ vom 14.10 .1988 , S. 54-75

o. v.: Textkommunikation per Telefax und Teletex, in: Deutsche Handwerks Zeitung $\mathrm{Nr} .11$ vom 9. Juni 1989

o.v.: Telekommunikationslabor soll kleine und mittlere Unternehmenberaten, in: VDI Nachrichten vom 30.06.89

o.v.: Post will bis Ende 1990 in 300 städten ISDN-Anschluß bieten, in: Frankfurter Allgemeine Zeitung Nr. 195 vom 24 . August 1989 , S. 5

o.v.: Mütel eröffnet ISDN-Beratungscenter in München, in: ISDN report Mai 1989

o.v.: Informationen aus Datenbanken - Beschaffung - Beratung, Informationsblatt der Innovations- und Technologieberatungsstelle der IHK-Frankfurt, Frankfurt 1989

๑.v.: Informationsblatt MüTel e.v., München 1989

o.V.: ISDN-Kommunikationsmöglichkeiten für die zukunft Telekommunikationsberatung für den Mittelstand, in: Deutsche Handwerks Zeitung vom 21. April 1989

o.v.: Neue Chancen durch Selbstwahl im Glasfasernetz, in: IHK - Mitteilungen vom 15.11.89

o.v.: Der Minitel erobert in Frankreich die privaten Haushalte - Der elektronische Auftraggeber gehört schon zum täglichen Leben/Das Gerät ist gratis, berechnet wird die Benutzung, in: Frankfurter Allgemeine Zeitung Nr. 42 vom 19.02 .1990$, S. 14

Quentmeier-vieregge, R.; storbeck, D.: Möglichkeiten und Hindernisse der Telematik am Beispiel eines Mittelzentrums: Herford, in: Akademie für Raumforschung und Landesplanung (Hrsg.): Räumliche Wirkungen der Telematik, Hannover 1987, S. $417-469$

Raumordnungsgesetz vom 8. April 1965, in: BGBl. I, S. 306

Ratzke, D.: Handbuch der Neuen Medien, Stuttgart 1982

Reese, J.: Die Bedeutung der neuen Fernmeldedienste für Nordhessen, Gutachten im Auftrag der Hessichen Staatskanzlei, Kassel 1986

Reon, K.: ISDN - Alles aus einer Dose mit begrenztem Nutzen, in: Frankfurter Allgemeine Zeitung Nr. 89 vom 17. April 1990, S. T 1 
Richter, H.: Investitionshilfen - Wie man Kommunikationstechnik mit öffentlichen Zuschüssen optimal finanziert, in: cogito, Heft 4, 1987, S. 54-57

Richtlinien für das ERP-Regionalprogramm 1986/87: Richtlinien für das ERP-Regionalprogramm 1986/87, abgedruckt in: Deutscher Bundestag: Siebzehnter Rahmenplan der Gemeinschaftsaufgabe "Verbesserung der regionalen Wirtschaftsstruktur", Bundestagsdrucksache 11/2362, Bonn 1988, S. 204

Rittenbruch, K.: Zur Anwendbarkeit der Exportbasis-Konzepte im Rahmen von Regionalstudien, Schriften zu Regional- und Verkehrsproblemen in Industrie- und Entwicklungsländern, Band 4, Berlin 1968

Rürup, B.; Hansmeyer, K.: Staatswirtschaftliche Planungsinstrumente, Düsseldorf 1984

Rürup, B.; Körner, H.: Finanzwissenschaft: Grundlagen der öffentlichen Finanzwirtschaft, Düsseldorf1985

Rürup, B.: Wirtschaftliche und gesellschaftliche Perspektiven der Bundesrepublik Deutschland, München 1989

8achs, L.: Angewandte Statistik - Statistische Methoden und ihre Anwendungen, 5. Auflage, Berlin, Heidelberg, New York 1978

8andvoB, J.: Fernarbeit: Formen und Potentiale dezentralisierter Arbeit im Lichte qualifikations- und regionalpolitischer Aspekte, Frankfurt u.a. 1989

sauga, M.: Gezänk über Gebühr, in: WirtschaftswocheSpecial-Supplement, $\mathrm{Nr}$. 3/88 vom 11.3.1988, S. 24-32

8chäfer, D.: Industrielle Ausstattung, Unternehmenspolitik und technisch-wissenschaftliche Infrastruktur als Voraussetzung einer innovationsorientierten Regionalpolitik, in: Informationen zur Raumentwicklung, 7, 1978, S. $489 \mathrm{ff}$

schambeck, H.: Unterstützung für den Mittelstand, in: Frankfurter Allgemeine Zeitung Nr. 241 vom 17.10.89, S. B8

Bchanz, G.: Kriterien zur Bestimmung der Größe des Forschungsbudgets in Unternehmungen der Industriegruppe Elektrotechnik, in: Zeitschift für betriebswirtschaftliche Forschung Nr. 2, 1972

scharlach, c. u.a.: Aspekte der Telekommuniaktiosentwicklung im Landes Rheinland-Pfalz, Deutsche Telepost Consulting GmbH (Hrsg.), Bonn, Mainz 1987

8charpf, F., schnabel, F.: Steuerungsprobleme der Raumplanung, in: Bruder, W.; Ellwein, W. (Hrsg.): Raumordnung und staatliche Steuerungstätigkeit, Opladen 1980, S. 12-57 
schiffer, R.: Konzeption der Telekommunikationsnetze und Planung für das Land Rheinland-Pfalz, in: Deutsche Telepost Consulting GmbH (Hrsg.): Aspekte der Telekommunikationsentwicklung im Land Rheinland-Pfalz, Bonn, Mainz 1987, Teil I

8chinagl, W.: Neue Dienste der Deutschen Bundespost, Fernmeldetechnisches Zentralamt der Deutschen Bundespost (Hrsg.), Darmstadt 1987

8chliebe, K.; Hillesheim, D.: Das Standortverhalten neuerrichteter und verlagerter Industriebetriebe im Zeitraum von 1970 bis 1979, in: Informationen zur Raumentwicklung, Heft 11,1980 , S. 613-620

8chmidt, B.: Bildschirmtext ist immer noch kein Medium für den privaten Markt, in: Frankfurter Allgemeine Zeitung Nr. 230 vom 4. Oktober 1989

8chmidt, E.: Interesse an neuem Fernmeldedienst ist noch gering - ISDN überfordert den Mittelstand, in: VDINachrichten Nr. 21 vom 26. Mai 1989, S. 22

schnöring, T.: Wirkungen der Telekommunikationsentwicklung auf Anwender, in: Deutsche Telepost Consulting $\mathrm{GmbH}$ (Hrsg.): Aspekte der Telekommunikationsentwicklung im Land Rheinland-Pfalz, Bonn, Mainz 1987, Teil IV

Bchnöring, T.: Telekommunikationsgebühren und Raumordnung, in: Akademie für Raumforschung und Landesplanung (Hrsg.): Räumliche Wirkungen der Telematik, Hannover 1987, S. 135156

schnöring, T.: Telekommunikationsgebühren und Raumordnung, Diskussionspapiere des Wissenschaftlichen Instituts für Kommunikationsdienste der Deutschen Bundespost, $\mathrm{Nr} .23$, Bad Honnef 1987

schnöring; T., Thomas, K.:Regionalpolitische Aspekte beim Angebot von Telekommunikationsdiensten, in: Bundesminister Schwarz-Schilling, Florian, W. (Hrsg.): Jahrbuch der Deutschen Bundespost 1985, Bad Windsheim 1985, 551-577

schön, H.: ISDN und ökonomie, in: Schwarz-Schilling, C., Florian, W. (Hrsg.): Jahrbuch der Deutschen Bundespost 1986, Bad Windsheim 1986, S. 9-50

8chröder, K.: "Technologieparks" in Niedersachsen - Eine Analyse der Ziele, Konzepte und Implementierung, Göttingen 1985

Bchrumpf, H.: Technologieparks als Instrument der kommunalen Wirtschaftsförderung, Bochum 1984

8chütte, G.: Neue Technik, Raumordnung und Städtebau: Mögliche Auswirkungen von Telekommunikation und Mikroprozessoren auf Planung und Entwicklung von Stadt- und Regional- 
strukturen - Tagungsbericht -, in: Raumforschung und Raumordnung, Heft 4, 41. Jahrgang, 1983 S. 169-173

scientific Control system GmbH (Hrsg.): Studie für einen "Telekommunikationsentwicklungsplan" für das Land Nordrhein-Westfalen, Essen 1984

selig, J.: EDV-Mangagement, Eine empirische Untersuchung der Entwicklung von Anwendungssystemen in deutschen Unternehmen, Berlin, Heidelberg, New York, Tokyo 1986

sieber, L.: Subventionen, Erlangen, Nürnberg 1968

siegel, S.: Nichtparametrische statistische Methoden, 2. Auflage, Eschborn 1985

spannowsky, W.: Der Handlungsspielraum und die Grenzen der regionalen Wirtschaftsförderung des Bundes, Berlin 1987

spehl, H. U.a.: Regionale Wirtschaftspolitik und regionale Entwicklungsplanung, Bonn 1981

8pehl, H.: Thesenpapier, in: Regionalpolitik 2000 - Probleme, Ziele, Instrumente, Schriftenreihe der Wirtschafts- und Gesellschaftswissenschaftlichen Gesellschaft Trier e.V., Trier 1984

8pehl, H.: Räumliche Wirkungen der Telematik, in: Raumforschung und Raumordnung Nr.4-6, 43. Jahrgang, 1985, S. 254269

8pehl, H.: Entwicklung der Telematik im Trierer Raum überwiegen die Chacen oder die Probleme?, in: Spehl, H.; Messerig-Funk, B. (Hrsg.): Telematik Trier, Trier, 1986, S. 105-117

Spehl, H.; Messerig-Funk, B.: Chancen und Probleme der wirtschaftlichen Nutzung der Telematik in einer ländlichen Region: Trier, in: Akademie für Raumforschung und Landesplanung (Hrsg.): Räumliche wirkungen der Telematik, Forschungs- und Sitzungsberichte Band 169, Hannover 1987, S. 417-446

8tahl, E.: Regionale Aspekte in der Forschungs- und Technologiepolitik, in: Innere Konolisation, Band 4, Bonn 1981

sternberg, R.: Technologie- und Gründerzentren als Instrument kommunaler Wirtschaftsförderung - Bewertung auf der Grundlage von Erhebungen in 31 Zentren und 177 Unternehmen, Dortmund 1988

stiens, F.: Vorausgesagte Entwicklungen und neue Strategien für den ländlichen Raum, in: Informationen zur Raumentwicklung, Heft 1/2, 1977 
stöhr, W.: Ansätze zu einer neuen Fundierung der Regionalpolitik, in: Jahrbuch der Regionalwissenschaft, 0.0. 1984, S. $7-28$

storbeck, D.: Telekommunikation und siedlungsstruktur, in: $\mathrm{ArCh}+\mathrm{Nr} .75 / 76,1984$, S. 53-55

8torbeck, D., Lücke, M.: Die gesellschaftspolitische Relevanz regionalpolitischer ziele, in: Ausgeglichene Funktionsräume, Hannover 1975, S. 19-62

8T8C, InC.: STATGRAPHICS - USER'S GUIDE, Maryland, Rockville 1988

8ydow, J.: Organisationsspielraum und Büroautomation, Berlin/New York 1985

Tennagels, P.: Instrumentarium der regionalen wirtschaftspolitik, Bochum 1980

Tenzer, G. (Hrsg.): Büroorganisation - Bürokommunikation, Mittel zur steigerung der Produktivität, Heidelberg, 1984

Tesdorf, J.: Regionalplanung in Baden-Würtemberg: In der Zerreißprobe, in: Innere Kolonisation, 4/1978

Tiemann, U.: Anwendungen neuer Informations- und Kommunikationstechnologien im Ruhrgebiet, in: Spehl, H.; MesserigFunk, B. (Hrsg.): Telematik Trier, Ergebnis eines Symposiums, Beiträge aus Forschung und Praxis, Band I, Trier, 1986 , S. 91-104

Tuchtfeldt, E.: Infrastrukturinvestitionen als Mittel der Strukturpolitik, in: Jochimsen, R.; Simonis, U. (Hrsg.): Theorie und Praxis der Infrastrukturpolitik, Schriften des Vereins für Socialpolitik, Band 54, Berlin 1970, S. $125 \mathrm{ff}$

Türke, K.: Die Herausforderung neuer Informations und Kommunikationstechniken an die Raumplanung: Das elektronische Dorf - Die verkabelte Stadt, in: Akademie für Raumforschung und Landesplanung (Hrsg.): Arbeitsmaterial $\mathrm{Nr} .81$, Hannover 1984 , S. 5-28

Ulrich, E.; Weizäcker, von C.: Fehlerfreundlichkeit als evolutionäres Prinzip, in: Wechselwirkungen, Nr. 29, 1986, S. $12 \mathrm{ff}$

Wagner, P.: Gratwanderung oder Durststrecke - Probleme mit ISDN, in: Frankfurter Allgemeine Zeitung Nr. 241 vom 17. Oktober 1989 , S. B26

Weitzel, G. u.a.: Post- und Fernmeldegebühren in ausgewählten Wirtschaftsbereichen, Ifo-studie zur Verkehrswirtschaft Band 15, München 1983 
Weltz, F.: Veränderung der Leitungsfunktion durch neue Technik, in: Office Management 5/1985, S. 468-470

Weltz, F.; Bollinger, H.: Dezentralisierung und Integration: Zauberformel der Büroarbeit, in: office Management $3 / I-1987$, S. $52-56$

Wettman, R. u. a.: Deglomerationspolitiken in den Europäischen Gemeinschaften, Berlin 1978

Wild, K.-P.: Stellung und Aufgabend der Länder, in: Eberstein, H. (Hrsg.): Handbuch der regionalen Wirtschaftsförderung, Abschnitt 1 VI A, Köln 1971, S. 2

winand, U. u.a.: Telekommunikation in Hessen, Gesellschaft für Information und Dokumentation mbH (Hrsg.), Frankfurt 1986

Winkelhage, F.: Wirkungen neuer Medien auf Arbeitsteilung und Arbeitsabläufe in Wirtschaft und Verwaltung, in: Informationen zur Raumentwicklung, Heft 3, 1982, S. 201-212

Wirtschaftsjunioren der Industrie- und Handelskammer Darmstadt mit Unterstützung der Stadt Darmstadt (Hrsg.): Handbuch für Technologietransfer, Darmstadt 1989

Wolf, F.: Möglichkeiten einer wirksameren Raumordnungs- und Regionalpolitik, in: Bruder, W. und Ellwein, T. (Hrsg.): Raumordnung und staatliche steuerungsfähigkeit, Opladen 1980 , S. 195-215

wolf, F.: Wirtschaftsschwache Gebiete: Politik der offenen Hand oder strategie der staatlich gestützen regionalen Eigenentwicklung?, in: Raumforschung und Raumordnung, Heft 5-6, 45. Jahrgang, Köln 1987, S. 200-207

zimmermann, H.: Öffentliche Ausgaben und regionale Wirtschaftsentwicklung, Basel, Tübingen 1970 
Stephan Russ - 978-3-631-75107-7

Downloaded from PubFactory at 01/11/2019 08:15:47AM

via free access 
Anhang 
Stephan Russ - 978-3-631-75107-7

Downloaded from PubFactory at 01/11/2019 08:15:47AM

via free access 
Hinweis: Die Prozentangeben beziehen sich aut die Grundgesamtheit der beantworteten und auswert baren Fragebogen. Die Summe der Prozentangaben pro Antwort muß nicht zwangsiauflg $100 \%$ orggben, da einerseits Mehrfachnennungen mógliche sind (Summe über 100\%) und andererseits vereinzelt Fragen nicht beantwortet wurden.

\section{Ergebnisse der Hauptuntersuchung:}

"Fragebogen zur Analyse des Einflusses telekommunikativer Techniken auf die Standortwahl"

Bitto Zutrelfondes ankrouzen [X] bzw. ausfollen. Mohrlachnennungen sind loillwoiso moglich! Zusdtzlicho Angabon in den vorgegebenen Froirdumen sind orwunscht. Sollten sio oine frago nicht beantworton konnen, uberspringen sio diese bitto.

1. Wo liegt der Standort Ihres Betriebes?

Bitto krouzen sio das ontsprochendo fold an

Großraum Frankfurt

Großraum Kassel
$52.6 \times$ Gropraum Gleßen

$18,4 \times$ Gropraum Fulda
$23,3 \%$

$7.8 \times$

2. Wieviel Beschäftigte hat Ihr Unternehmen?

Bitto krouzen sio dio entsprechendo Gropenkslogorio an

\begin{tabular}{|c|c|c|c|}
\hline $01-09$ & $\sim \sim$ & $70-79$ & $1.7 \times$ \\
\hline $10-19^{-}$ & & $80-89$ & \\
\hline $20-29$ & $69 \%$ & $90-99$ & \\
\hline $30-39$ & & $100-199$ & \\
\hline $0-49$ & $2.6 \times$ & 200 u.m. & 1.0 \\
\hline
\end{tabular}

3. Wie hoch war der Umsatz Ihres Betriebes? Bitto oventuall abschdton

1987: DM

$$
000000-<500.000
$$
$500.000-<1.000 .00$

1988: $1.000 .000-<3.000 .000$ $3.000 .000-<5.000 .000$ DM

4. Welcher Branche gehört Ihr Unternehmen an?
$37,1 \times \square \quad$ Unternehmensberatungen
$20.7 \times$
Soltware - Firmen
$15.5 \times \square$
Elnzelhandel
$26.7 \times \square$
EDV - Vertrieb
Maschinenbau

Sonstige: 
5. Hat Ihr Unternehmen mehr als einen Standort?

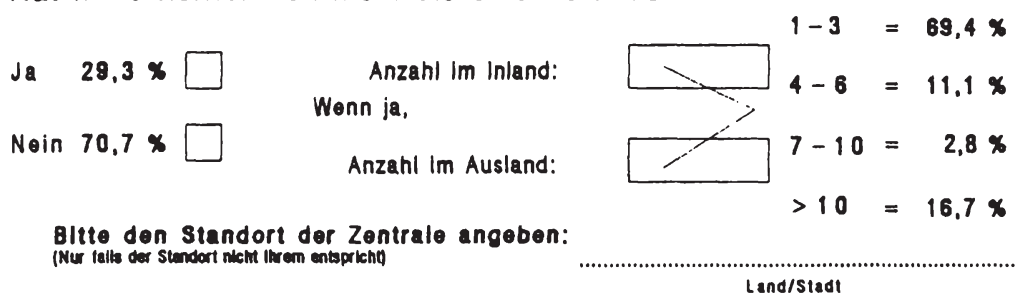

6. Welcher Kategorie gehört Ihr Betrieb an? Bitto krouzen sio die Kategorie an, der Slo zugehdren

88.1 $\times$ Elgenstándige Unternehmung

Zweigstelle/Fillale

$7.0 \times$ Zentralo

Nlederlassung/Gescháftsstelle

Sonstige

Bitite angeben

7. Welche Funktion nehmen Sie in Ihrem Betrieb wahr?

8. Setzen Sie Mikro - oder Personalcomputer ein? $1-3=50,0 \times$

Ja

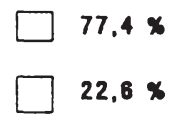

Wenn ja, Anzahl:

Noin

$22,6 \times$

$4-6=22,2 \times$

$7-10=13.9 \%$

$>10=13,9 \times$

9. Werden in Ihrem Unternehmen eigene Forschung - und Entwicklungs tătigkeiten vorgenommen?

\begin{tabular}{|c|c|c|c|}
\hline $\mathrm{Ja}$ & $58,1 \times$ & $\begin{array}{l}\text { Wenn Ja, bltte ca. Prozentsatz } \\
\text { der FuE-Kosten vom Umsatz }\end{array}$ & \\
\hline Noin & $43,9 \times$ & angeben: & $\%$ \\
\hline
\end{tabular}

10. Wie hoch sind die monatlichen Gebühren für die von Ihnen genutzten Dienste?

bitto oventuell abschatzon
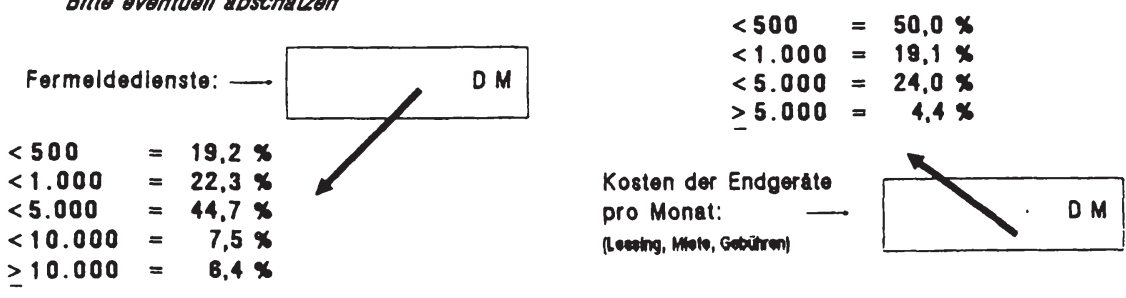
11. Wie steht Ihr Betrieb zu folgenden Informations - und Kommunikations diensten? Jemeils in $\%$ der Zeile

\begin{tabular}{|c|c|c|c|c|c|c|c|}
\hline $\begin{array}{l}\text { Bitte krouzen Sie zu jodem } \\
\text { Dienst ein Fold an }\end{array}$ & & & $\begin{array}{l}\text { Elnsatz } \\
\text { geplant }\end{array}$ & $\begin{aligned} \text { Bek } \\
\text { ni }\end{aligned}$ & $\begin{array}{l}\text { Isatz } \\
\text { ant }\end{array}$ & $\begin{array}{l}\text { Nicht } \\
\text { bokannt }\end{array}$ & $M$ \\
\hline Funkfornsprochon & 26.2 & 12.8 & & 46.8 & 14.6 & & 1. \\
\hline Eurosignal & 13.1 & 4,0 & & 70.0 & 13.1 & & 1. \\
\hline Cltyrut & - & 2.3 & & 72.7 & 25,0 & & \\
\hline GEDAN (Anrufweiterschaltung) & 9,3 & 6.2 & & 48,5 & 38,1 & & \\
\hline Service 130 & 2.1 & 1.1 & & 47.4 & 49.5 & & \\
\hline Sprachbox & - & & & 34,0 & 66,0 & & \\
\hline Tolex & 41.3 & 1,0 & & 54.8 & 2.9 & & \\
\hline Tolotex & 13.0 & 4.0 & & 78.0 & 5.0 & & 1.2 \\
\hline Btx & 14,3 & 12.2 & & 68.4 & 5.1 & & 1. \\
\hline Tolobox & - & 5,4 & & 54.8 & 39,8 & & o. \\
\hline - electronic mall & 9,5 & 11,6 & & 47.4 & 31.6 & & 1. \\
\hline Datenabertr. Im Teletonnetz & 36,8 & 12,6 & & 32.6 & 17.9 & & .8 \\
\hline Datex - L & 8.6 & 1.1 & & 50.5 & 39,8 & & 0.8 \\
\hline Datex - $P$ & 17.9 & 9,5 & & 39.0 & 33.7 & & 1.1 \\
\hline$H \in D$ & 4.4 & 1.1 & & 23.1 & 71.4 & & 0.4 \\
\hline Temex & - & 1.1 & & 23.1 & 75.8 & & \\
\hline Tolofax & 72,8 & 18.8 & & 8,0 & 2.7 & & 2. \\
\hline Blidtelefon & & 3,2 & & 85.1 & 11.7 & & 0. \\
\hline Videokonferenz & & 2.1 & & 85.1 & 12,8 & & \\
\hline
\end{tabular}

12. Bei welchem Endgerätepreis würden Sie folgende Telekommunikations dienste anschaffen?

bitto for jodes Endgerdt oinen Prois ankrouzen

\begin{tabular}{|l|c|c|c|c|c|c|c|c|c|c|}
\hline Komfort-Telefon & on & 0 & 25 & 50 & $75 \times 100$ & 200 & 300 & 400 & 500 \\
\hline Tolex & on & 0 & 250 & 500 & $\# 0$ & 1000 & 2000 & 3000 & 4000 & 5000 \\
\hline Toletex & on & 0 & 250 & 500 & $75 \times$ & 1000 & 2000 & 3000 & 4000 & 5000 \\
\hline Telefax & oin & 0 & 250 & 500 & 750 & 1000 & $2000 \times 3000$ & 4000 & 5000 \\
\hline Bix & on & 0 & 250 & 500 & $\# 0$ & 1000 & 2000 & 3000 & 4000 & 5000 \\
\hline
\end{tabular}


13. Wie bewerten Sie Ihren momentanen Standort?

bitto krouzon sio pro Zoilo oin Kastchon an

\begin{tabular}{|c|c|c|c|c|c|c|}
\hline & $\begin{array}{l}\text { sehr } \\
\text { gut }\end{array}$ & gut & $\begin{array}{l}\text { befrle - } \\
\text { digend }\end{array}$ & $\begin{array}{l}\text { aus - } \\
\text { relchend }\end{array}$ & $\begin{array}{l}\text { mangel - } \\
\text { haft }\end{array}$ & $\begin{array}{l}\text { nicht zu } \\
\text { beant - } \\
\text { worten }\end{array}$ \\
\hline Reservoir an Fôhrungskaften & $10,6 \%$ & $26,5 \%$ & $16,8 \times$ & $11.5 \%$ & $17,7 \%$ & $16,8 \times$ \\
\hline Reservoir an qualifizierten Fachkraften & $10,4 \%$ & $24,4 \%$ & $19,1 \times$ & $12.1 \times$ & $31,3 \times$ & $2,6 \times$ \\
\hline Reservoir an Hilfskraften & $10.5 \%$ & $27.2 \%$ & $32,5 \times$ & $18,4 \%$ & $3.5 \%$ & $7,9 \times$ \\
\hline Lohnkostenniveau & $6,4 \%$ & $21,1 \times$ & $45.0 \times$ & $11,0 \times$ & $8,2 \%$ & $7,3 \times$ \\
\hline Nahe zum Absatzmarkt & $20.4 \%$ & $42,5 \%$ & $18,6 \%$ & $6.2 \%$ & $3,5 \%$ & $8,9 \times$ \\
\hline Nahe zum Beschaffungsmarkt & $7,2 \%$ & $38.7 \times$ & $22,5 \times$ & $9.9 \times$ & $5.4 \%$ & $16.2 \times$ \\
\hline Nâhe zur Konkurrenz & $9,7 \%$ & $37.2 \times$ & $21.2 \times$ & $13,3 \%$ & $2.7 \times$ & $15.9 \times$ \\
\hline Nâhe zu Forschungseinrlchtungen & $9,9 \%$ & $24,3 \times$ & $17.1 \times$ & $14,4 \times$ & $9,0 \%$ & $25.2 \%$ \\
\hline Nahe zu Behơrden & $11,4 \times$ & $43,0 \%$ & $20,2 \times$ & $17,5 \times$ & $1,8 *$ & $6,1 \times$ \\
\hline Angebot an Gewerbeflachen/Büros & $8,7 \%$ & $25,2 \%$ & $19,1 \times$ & $20,0 \times$ & $16.5 \times$ & $10,4 \times$ \\
\hline Kosten for Gewerbeflachen/Baros & $4,4 \%$ & $20,0 \%$ & $21,7 \times$ & $20,0 \times$ & $23,5 \times$ & $10,4 \times$ \\
\hline Erwoiterungsmoglichkeiten & $4.4 \%$ & $34,0 \times$ & $13,0 \times$ & $16,5 \times$ & $28.7 \%$ & $3,5 \times$ \\
\hline Unbörokratisches Verhalten der Kommune & $1,8 x$ & $8,0 \%$ & $24.1 \times$ & $24,1 \times$ & $21.4 \times$ & $20.5 \times$ \\
\hline Finanzielle Forderung durch dle Kommune & & $1,8 \%$ & $8,0 \%$ & $10.7 \times$ & $44,6 \%$ & $34,8 \times$ \\
\hline Finanzielle Forderung durch Bund/Land & & $4,5 \%$ & $10,8 \%$ & $13.5 \%$ & $36.9 \times$ & $34,2 \times$ \\
\hline Anbindung an Straßen, Autobahnen & $48,7 \%$ & $31.1 \%$ & $10,4 \%$ & $5.2 \times$ & $2,6 \%$ & $1.7 \%$ \\
\hline Anbindung an Bundesbahn, Elsenbahn & $33,8 \%$ & $24,4 \%$ & $13,0 \%$ & $8.7 \%$ & $16,5 \%$ & $3,5 \%$ \\
\hline Zeltpunkt der Anbindung an ISDN - Netz & $4,6 \%$ & $18,7 \%$ & $8,3 \times$ & $7,4 \%$ & $10,2 \times$ & $52,8 \times$ \\
\hline $\begin{array}{l}\text { Verfügbarkeit neuer telekommunikativer } \\
\text { Techniken }\end{array}$ & $10,8 \%$ & $27,0 \%$ & $19,8 \times$ & $5,4 \times$ & $6,3 \times$ & $30,6 \times$ \\
\hline Image/Umield & $13,2 \%$ & $37.7 \%$ & $25.4 \%$ & $10.5 \%$ & $6.1 \%$ & $7.0 \%$ \\
\hline $\begin{array}{l}\text { Nahe zu Kultur - und Freizeiteinrich - } \\
\text { tungen }\end{array}$ & $18,4 \%$ & $36,0 \%$ & $24,6 \%$ & $11.4 \%$ & $5,3 \%$ & $4,4 \%$ \\
\hline $\begin{array}{l}\text { Nahe zu medizinischen Versorgungsein - } \\
\text { rlchtungen und sozialen Dlensten }\end{array}$ & $28,3 \%$ & $45,6 \%$ & $17.5 \%$ & $6.1 \%$ & $1,8 \%$ & $2,8 \times$ \\
\hline Wohnstandortwünsche von Angestellten & $7.8 \%$ & $28,1 \%$ & $28.7 \times$ & $17.4 \times$ & $3.5 \%$ & $18,5 \%$ \\
\hline Sonstige: & & & & & & \\
\hline 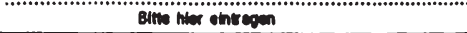 & & & & & & \\
\hline
\end{tabular}


14. Wie schätzen Sie die Bedeutung der folgenden Fernmeldedienste für Ihren Betrieb ein?

sitto lor joden Dlonst oin Kdstchen snkrouzon

\begin{tabular}{|c|c|c|c|c|c|c|}
\hline & & $\begin{array}{l}\text { von } \\
\text { großer } \\
\text { Bedeutung }\end{array}$ & $\begin{array}{l}\text { von } \\
\text { Bedeutung }\end{array}$ & $\begin{array}{l}\text { von } \\
\text { geringer } \\
\text { Bodeutung }\end{array}$ & $\begin{array}{l}\text { keine } \\
\text { Bedeutung }\end{array}$ & $\begin{array}{l}\text { nicht } \\
\text { zu } \\
\text { bourteiler }\end{array}$ \\
\hline Telofon & $96.6 \times$ & $3.4 \times$ & & & & \\
\hline Telox & $7.2 \%$ & $22,5 \%$ & $27.9 x$ & $36.0 \times$ & $6,3 \%$ & \\
\hline Telotex & $2.8 \times$ & $10,1 \times$ & $28,4 x$ & $41,3 \times$ & $17.4 \times$ & \\
\hline $81 x$ & $2.8 \%$ & $10.1 \times$ & $35,8 x$ & $35.8 \times$ & $15.6 \times$ & \\
\hline Tolofax & $74,6 \%$ & $14.9 \times$ & & $7.0 \times$ & $3.5 \%$ & \\
\hline $\begin{array}{l}\text { Datenabertragungs - } \\
\text { dienste }\end{array}$ & $28,6 \%$ & $34,8 \times$ & 8,0 & $13,4 \times$ & $\square 15,2 \times$ & \\
\hline
\end{tabular}

15. Welche Gründe hindern Sie daran, neue Telekommunikationstechniken in Ihrem Betrieb einzusetzen? Bltse dlo verschlodenon Grunde ankrouzen (Mehrlachnennungen moglich)

- Nutzung zu komplizlert

- Information unzurelchend

- Dienste bringen keinen Nutzen für den Botrieb

- Anschlußdichto / Fláchendeckung noch zu gering

- Mitarbeiter sind for dle Nutz. noch nicht qualiftzert

- Kelne

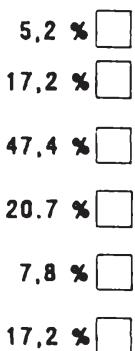

$17.2 \times$
\& der Nennungen je Grund

- Gebohrenstrukturen sind unklar

- Laufende Gebohren sind zu hoch

- Anfangsinvestitlonen/

Einfohrungskosten sind zu hoch

- Keine Bereitstellung eines Anschlusses in absehbarer Zeit

- Bereitstellungsmóglichkeiten der Endgerate noch nicht geklart

- Weltere Gründe *
$18,1 \%$

$25.9 \%$

$25,0 \times \square$

$8,6 \times \square$

$12.1 \times \square$

$0.8 \times$

- Gronde bitte hler angeben

16. Wie sind Sie auf den jetzigen Standort gekommen? bitto krouzon sio dio entsprechendon Kistchon an (Wohrtachnennungen moglich)

- Persónliche Kenntnis

- Verbánde (IHK, VDMA, o.e. )

- Zeitung, Zeitschriften, Bocher

- Sonstlge Informationsquellon*
$78.8 \times$

$2.0 \times \square$

$6.1 \%$
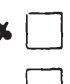

- Vertreter der Gemeinde/Stadt

- Entscheidung der firmenz.

- Koine Kenntnis
$2,0 \times \square$

$10.1 \times \square$

\section{\% der Antworten ohne Mehrtachnennungen}

-Bitte angeben 
17. Welchen Einfluß hatten die im folgenden aufgeführten Faktoren auf die Auswahl des jetzigen Standorts?

Bitto zu jodom Faktor oin Kástchon ankrouzen

\begin{tabular}{|c|c|c|c|c|c|c|}
\hline & $\begin{array}{l}\text { sehr } \\
\text { großen }\end{array}$ & groben & $\begin{array}{l}\text { mitt- } \\
\text { leren }\end{array}$ & $\begin{array}{l}\text { weniger } \\
\text { großen }\end{array}$ & keinen & $\begin{array}{l}\text { nicht zu } \\
\text { beant - } \\
\text { worten }\end{array}$ \\
\hline Reservoir an Fuhrungskraften & $4,2 \times$ & $8,4 \%$ & $6,3 \%$ & $14,7 \%$ & $49,5 \%$ & $16,8 \%$ \\
\hline Reservoir an qualifizierten Fachkráften & $3,2 \%$ & $15,8 \%$ & $12,6 \%$ & $12,6 \%$ & $43,2 \%$ & $12,6 \%$ \\
\hline Reservoir an Hilfskraften & $5,3 \%$ & $14,7 \%$ & $12,6 \%$ & $10,5 \%$ & $45,3 \%$ & $11,6 \%$ \\
\hline Lohnkostenniveau & $4,2 \%$ & $8,4 \%$ & $14.7 \%$ & $16.8 \%$ & $44.2 \%$ & $11,6 \%$ \\
\hline Nahe zum Absatzmarkt & $20,6 \%$ & $22,7 \%$ & $13,4 \%$ & $7.2 \%$ & $24,7 \%$ & $11,3 \%$ \\
\hline Năhe zum Beschaffungsmarkt & $2.1 \%$ & $12,8 \%$ & $14.9 \%$ & $10,6 \%$ & $44,7 \%$ & $14,9 \%$ \\
\hline Nâhe zur Konkurrenz & $3,2 \%$ & $8,5 \%$ & $11.7 \%$ & $11.7 \%$ & $50,0 \times$ & $14,9 \%$ \\
\hline Năhe zu Forschungseinrichtungen & $2.2 \%$ & $3,2 \%$ & $9,7 \%$ & $11.8 \%$ & $55.9 \%$ & $17.2 \%$ \\
\hline Náhe zu Behơrden & & $2,2 \%$ & $9.7 \%$ & $15,1 \%$ & $60.2 \%$ & $12,9 \%$ \\
\hline Angebot an Gewerbefláchen/Büros & $5,4 \%$ & $19,4 \%$ & $14,0 \%$ & $10.8 \%$ & $35,5 \%$ & $15,1 \%$ \\
\hline Kosten fūr Gewerbefláchen/Būros & $5,3 \%$ & $15,8 \times$ & $17.9 \times$ & $13,7 \%$ & $34.7 \times$ & $12,6 \%$ \\
\hline Erweiterungsmóglichkeiten & $4.3 \times$ & $12,8 \%$ & $20,2 \%$ & $18,1 \%$ & $33,0 \%$ & $11,7 \%$ \\
\hline Unbūrokratlsches Verhalten der Kommune & $1,1 \%$ & $1,1 \%$ & $7,5 \%$ & $14,0 \%$ & $61,3 \%$ & $15.1 \%$ \\
\hline Finanzielle Forderung durch die Kommune & & & $7.5 \%$ & $10,8 \%$ & $63.4 \%$ & $18,3 \times$ \\
\hline Finanzielle Förderung durch Bund/Land & $1.1 \%$ & $4,3 \%$ & $6,5 \%$ & $9,7 \%$ & $60,2 \%$ & $18,3 \%$ \\
\hline Anblndung an Straßen, Autobahnen & $22,3 \%$ & $31,9 \%$ & $13,8 \times$ & $3.2 \times$ & $18.1 \%$ & $10,6 \%$ \\
\hline Anbindung an Bundesbahn, Elsenbahn & $9,5 \%$ & $22,1 \%$ & $20,0 \%$ & $11,6 \%$ & $27.4 \%$ & $9.5 \%$ \\
\hline Zeitpunkt der Anbindung an ISON-Netz & $1,1 \%$ & $1.1 \%$ & $4,3 \times$ & $8,6 \%$ & $62,4 \%$ & $22,6 \%$ \\
\hline $\begin{array}{l}\text { Verfogbarkeit never telekommunikatlver } \\
\text { Techniken }\end{array}$ & $2.2 \%$ & $3,3 \%$ & $12,1 \%$ & $8,8 \times$ & $56,0 \%$ & $17,6 \times$ \\
\hline Image/Umield & $5,2 \times$ & $27,1 \times$ & $20,8 \times$ & $7,3 \times$ & $28,1 \times$ & $11,5 \times$ \\
\hline $\begin{array}{l}\text { Nâhe zu Kultur - und Freizeltelnrich - } \\
\text { tungen }\end{array}$ & $1,1 \%$ & $7.5 \%$ & $18,3 \times$ & $19,4 \%$ & $41,9 \%$ & $11,8 \%$ \\
\hline $\begin{array}{l}\text { Nahe zu medizinischen Versorgungsein- } \\
\text { richtungen und sozlalen Diensten }\end{array}$ & $1.1 \%$ & $7,5 \times$ & $18,3 . x$ & $10,8 \%$ & $47,3 \%$ & $11,8 \times$ \\
\hline Wohnstandortwünsche von Angestellten & $4,3 \%$ & $9,7 \%$ & $17,3 \%$ & $9,7 \times$ & $48.4 \%$ & $10.8 \%$ \\
\hline
\end{tabular}


18. Weitere im Vorhergehenden nicht genannte Faktoren, die für Ihre Standortentscheidung von Bedeutung waren:

19. Welche Formen der Förderung neuer Telekommunikationstechniken würden einen Standort am stärksten aufwerten?

1. Realtransfers in Form von kostenlosen:

\begin{tabular}{|l|l|l|l|l|}
\hline $\begin{array}{l}\text { sehr } \\
\text { stark }\end{array}$ & stark & $\begin{array}{l}\text { mittel } \\
\text { stark }\end{array}$ & $\begin{array}{l}\text { wenlger } \\
\text { stark }\end{array}$ & $\begin{array}{l}\text { gar } \\
\text { nicht }\end{array}$ \\
\hline
\end{tabular}

\begin{tabular}{|l|l|l|l|l|l|}
\hline$>$ Informationsdienstlelstungen & $13.8 \times$ & $34.0 \times$ & $21.3 \times$ & $14.9 \times$ & $18.0 \times$ \\
\hline$>$ Beratungsdienstleistungen & $13.5 \times$ & $26.0 \times$ & $24.0 \times$ & $18.8 \times$ & $17.7 \times$ \\
\hline$>$ Ausbildungsdienstlelstungen & $8.4 \times$ & $30.5 \times$ & $25.3 \times$ & $17.9 \times$ & $17.9 \times$ \\
\hline
\end{tabular}

2. Finanzielle Anreize in Form von:

\begin{tabular}{|c|c|c|c|c|c|}
\hline $\begin{array}{l}>\text { Subventionen für Neugrandungen } \\
\text { bzw. Granderzentren }\end{array}$ & $13,8 \times$ & $30,8 \times$ & $20,2 \times$ & $11.7 \times$ & $23.4 \times$ \\
\hline $\begin{array}{l}>\text { Bereitstellung kostengünstiger } \\
\text { Gewerbeflache }\end{array}$ & $21.8 \times$ & $32,3 \times$ & $18,7 \times$ & $12.5 \times$ & $18.7 \times$ \\
\hline $\begin{array}{l}>\text { Zinsgünstigen Darlehen oder } \\
\text { Burgschaften }\end{array}$ & $24,2 \times$ & $31.8 \times$ & $18.8 \%$ & $9,5 \times$ & $17.9 \times$ \\
\hline $\begin{array}{l}\text { > Subventionen for Neuinvestitionen } \\
\text { im Berelch neuer Informations - } \\
\text { und Kommunikationstechnlken }\end{array}$ & $29,6 \times$ & $27.6 \%$ & $17.4 \%$ & $7.1 \times$ & $18.4 \times$ \\
\hline
\end{tabular}

3. Anregung + Forderung von Kooperationen In Form von:

\begin{tabular}{|c|c|c|c|c|c|c|}
\hline & 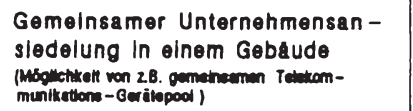 & $15.6 \times$ & $18.7 \times$ & $19,8 \times$ & $21,9 \times$ & $28,0 \times$ \\
\hline$>$ & $\begin{array}{l}\text { Gemeinsamer Gründung einer Un- } \\
\text { ternehmung durch mehrere Koope- } \\
\text { rationspartner, die als Teilhaber der } \\
\text { Unternehmung auftreten }\end{array}$ & $10.5 \times$ & $12,6 \times$ & $15,8 \times$ & $29.5 \times$ & $31.6 \%$ \\
\hline
\end{tabular}


20. Wie setzt sich das interne und externe Kommunikationsverhalten Ihres Unternehmens zusammen?

Bitto abschdizen und lor dio entsprechenden Dionsto den ca. $x$-Satz angeben

\section{Whittelwert aller Antworten}

\begin{tabular}{|l|}
\hline Personlicher Kontakt \\
\hline Telefon \\
\hline Brief \\
\hline Telex \\
\hline Tolofax \\
\hline Btx \\
\hline Datenobertragung \\
\hline Nicht aufgefohrt \\
\hline
\end{tabular}

\begin{tabular}{|c|}
\hline Intern \\
\hline $70 \%$ \\
\hline $21.3 \%$ \\
\hline $7.4 \%$ \\
\hline $8,6 \%$ \\
\hline $11.0 \%$ \\
\hline $12.0 \%$ \\
\hline
\end{tabular}

\begin{tabular}{|cc|}
\hline extern \\
\hline 35.3 & $\%$ \\
\hline 35.7 & $\%$ \\
\hline 14.7 & $\%$ \\
\hline 3.4 & $\%$ \\
\hline 11.4 & $\%$ \\
\hline 3.0 & $\%$ \\
\hline 8.0 & $\%$ \\
\hline & \\
\hline 100 & $\%$ \\
\hline
\end{tabular}

21. Inwieweit sind in Ihrem Unternehmen persönliche Kontakte durch neue Telekommunikationstechniken substituierbar?

Bitto Schdizwert in $\$$ pro Tolokommunikationsdionst joweils in - und extern ankrouzen

\begin{tabular}{|c|c|c|c|c|c|c|c|c|c|c|c|c|c|}
\hline \multirow{2}{*}{ ISDN-Telefon } & 0 & 102 & 20 & 30 & 40 & 50 & 60 & 70 & 80 & 90 & 100 & $\%$ & Intern \\
\hline & 0 & 10 & 20 & 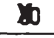 & 40 & 50 & 60 & 70 & 80 & 90 & 100 & $\%$ & Extern \\
\hline \multirow{2}{*}{ ISDN - Telex } & $\alpha$ & 10 & 20 & 30 & 40 & 50 & $\infty$ & 70 & 80 & 90 & 100 & $\%$ & Intern \\
\hline & $0 x$ & 10 & 20 & 30 & 40 & 50 & $\infty$ & 70 & 80 & 90 & 100 & $\%$ & Exterm \\
\hline \multirow{2}{*}{ ISDN - Teletex } & $0 x$ & 10 & 20 & 30 & 40 & 50 & $\infty$ & 70 & 80 & 90 & 100 & $\%$ & Intern \\
\hline & $0 x$ & 10 & 20 & 30 & 40 & 50 & $\infty$ & 70 & 80 & 90 & 100 & $\%$ & Extern \\
\hline \multirow[b]{2}{*}{ ISDN - Btx } & $x$ & 10 & 20 & 30 & 40 & 50 & $\infty$ & 70 & 80 & 90 & 100 & $\%$ & Intern \\
\hline & $0 x$ & 10 & 20 & 30 & 40 & 50 & $\infty$ & 70 & 80 & 90 & 100 & $\%$ & Extern \\
\hline \multirow{2}{*}{ ISDN - Telefax } & $0 x$ & 10 & 20 & 30 & 40 & 50 & $\infty$ & 70 & 80 & $\mathbf{9 0}$ & 100 & $\%$ & Intern \\
\hline & 0 & 10 & 20 & 200 & 40 & 50 & 60 & 70 & 80 & 90 & 100 & $\%$ & Extern \\
\hline \multirow{2}{*}{ Bildtelefon } & $0 x$ & 10 & 20 & 30 & 40 & 50 & 60 & 70 & 80 & 90 & 100 & $\%$ & Intern \\
\hline & 0 & 10 & $20 x$ & 30 & 40 & 50 & 60 & 70 & 80 & 90 & 100 & $\%$ & Extern \\
\hline \multirow{2}{*}{ videokonferenz } & $0 x$ & 10 & 20 & 30 & 40 & 50 & $\infty$ & 70 & 80 & 90 & 100 & $\%$ & Intem \\
\hline & 0 & 10 & $x_{20}$ & 30 & 40 & 50 & 60 & 70 & 80 & 90 & 100 & $\%$ & Extern \\
\hline \multirow[b]{2}{*}{ 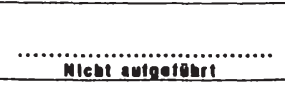 } & 0 & 10 & 20 & 30 & 40 & 50 & 60 & 70 & 80 & 90 & 100 & $\%$ & Intern \\
\hline & 0 & 10 & 20 & 30 & 40 & 50 & $\varpi$ & 70 & 80 & 90 & 100 & $\%$ & Extem \\
\hline \multicolumn{10}{|c|}{$\begin{array}{l}\text { Personliche Kontakte lassen sich nicht durch } \\
\text { noue Telekommunikationstechniken ersetzen }\end{array}$} & \multicolumn{4}{|c|}{$64,7 \%$} \\
\hline
\end{tabular}


22. Bitte geben Sie, falls gewollt, Ihre Firmenadresse und einen möglichen Ansprechpartner für eventuelle Rückfragen an.

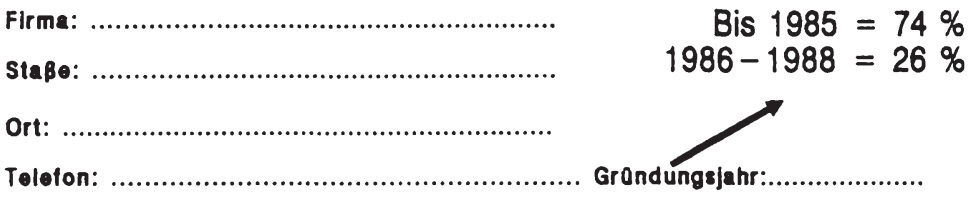

Ansprechpartner:

23. Wăren Sie grundsätzlich bereit, falls erforderlich an einer Fallstudie über neue Telekommunikationstechniken mitzuwirken?

Ja Noin $\square \quad \square 2,9 \%$

Ende des Frogebogene

Kritik und Kommentare zum Fragebogen:

Zum Schluß möchten wir uns noch einmal sehr herzlich für die Mūhe bedanken, die Sie sich bei der Beantwortung des Fragebogens gemacht haben.

Bitte solen sie so froundilch, den ausgefallten Fragobogen bis spatestens zum 16. Mai zurôckzusenden an:

Technische Hochschule Darmstadt

Professor Dr. Bert Rorup

Fachgoblet Volkswirtschaftslohro III

z.Hd. Herrn Rup

Fachbereich 1

Residenzschloß

6100 Dermstedt 


\section{SOZIALÖKONOMISCHE SCHRIFTEN}

Herausgegeben von Professor Dr. Bert Rūrup

Band 1 Marietta Jass: Erfolgskontrolle des Abwasserabgabengesetzes. Eln Konzept zur Erfassung der Gesetzeswirkungen verbunden mit einer empirischen Untersuchung in der $\mathrm{Pa}$ pierindustrie. 1990.

Band 2 Frank Schulz-Nieswandt: Stationāre Altenpflege und "Pflegenotstand" in der Bundesrepublik Deutschland. 1990.

Band 3 Helmut Böhme, Alois Peressin (Hrsg.): Sozialraum Europa. Die soziale Dimension des Europåischen Binnenmarktes. 1990.

Band 4 Stephan RuB: Telekommunikation als Standortfaktor für Klein- und Mittelbetriebe. Telekommunikative Entwicklungstendenzen und regionale Wirtschaftspolitik am Beispiel Hessen. 1991. 


\section{Michael Gedatus}

\section{Massenmedien, Kommunikationstechnologie und gesellschaftliche Entwicklung in der Dritten Welt Möglichkeiten und Grenzen von Satellitenkommunikation am Beispiel Indien}

Frankfurt/M., Bern, New York, Paris 1988. XIV, 619 S.

Europäische Hochschulschriften: Reihe 22, Soziologie. Bd. 161

ISBN 3-8204-1474-6

br./lam.DM 102.--/sFr. 85.--

Hinsichtlich der Anwendung von Kommunikationssatelliten in der Dritten Welt zum Zwecke zielgerichteter Entwicklungskommunikation sollen am Beispiel Indien Möglichkeiten und Grenzen des Technologieeinsatzes überprüft und entwicklungskommunikations-theoretischen Hypothesen gegenübergestellt werden. Aus der Falluntersuchung werden weiterführende Schlußfolgerungen abgeleitet. Dabei werden Konzepte und Strategien der Veränderung in wichtigen Sektoren der internationalen Beziehungen, gerade auch mit Blick auf die eigenständige Entwicklung von Dritte-Welt-Gesellschaften, aufgegriffen ("Weltkommunikationsordnung"). Der Band versteht sich als Beitrag zur Entwicklungskommunikationsforschung und -theorie.

Aus dem Inhalt: Die Dritte Welt - Kommunikationssoziologische Aspekte gesellschaftlicher Entwicklung; Indien - Kommunikation, Medien und Gesellschaft Entwicklungskommunikationsforschung - Internationale Kommunikationspolitik und Entwicklungsaussichten für die Dritte Welt in den 80er Jahren

"Nicht nur das entwicklungstheoretische Fundament, auch die technische Versiertheit des Autors, sein souveräner Umgang mit den Methoden der empirischen Sozialforschung und seine Methode, Hypothesen aufzustellen, Fakten zu präsentieren und dann vorsichtig zu werten, machen diese Untesuchung zu einem Meilenstein auf dem Wege zu einer entwicklungsrelevanten Kommunikationsforschung." Rainer Kabel in Medienwissenschaft

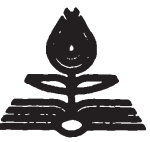

Verlag Peter Lang Frankfurt a.M. $\cdot$ Bern $\cdot$ New York $\cdot$ Paris Auslieferung: Verlag Peter Lang AG, Jupiterstr. 15, CH-3000 Bern 15 Telefon (004131) 321122, Telex pela ch 912651 , Telefax (004131) 321131 


\section{Rainer Böhme}

\section{Die informations- und kommunikationstechnische Industrie und Krisenüberwindung in der Bundesrepublik Deutschland Rahmenbedingungen und Formen in einer Modernisierungsindustrie und ihre Konsequenzen}

Frankfurt/M., Bem, New York, Paris, 1989. XV, 467 S., 4 Abb., 89 Tab.

Europäische Hochschulschriften: Reihe 31, Politikwissenschaft. Bd. 148

ISBN 3-631-42334-9

br. DM 102.--/sFr. 93.--

Die informations- und kommunikationstechnische Industrie wird auf wirtschaftspolitischer Ebene wie auch in vielen krisentheoretischen Ansätzen als wichtiges Element der Krisenüberwindung durch Modemisierung angesehen. Die strukturellen Bedingungen in dieser neu entstehenden Branche der Hochtechnologie besitzen damit beachtliche Relevanz für die sozio-ökonomische wie auch inbesondere für die industrielle Entwicklung in der Bundesrepublik Deutschland.

Die vorliegende Arbeit nimmt auf empirischer Basis eine Analyse der informations- und kommunikations-technischen Industrie vor. Es werden ausführlich die internationalen Strukturen des Industriezweiges einschließlich der diesbezüglichen Industriepolitik in wichtigen Ländern aufgedeckt, welche die entscheidenden Rahmenbedingungen darstellen. Mit der detaillierten Untersuchung der Branche in der Bundesrepublik sowie der entsprechenden staatlichen Industriepolitik - ergänzt durch die Betrachtung von Positionen zur Informations- und Kommunikationstechnik der gesellschaftlich relevantesten Gruppierungen - erfolgt die Herausarbeitung der nationalen Bedingungsfaktoren. Die internationalen und nationalen Branchenstrukturelemente zusammen erlauben eine erste Einschätzung der spezifischen Folgen von Modernisierung mit dieser Industrie für die Bundesrepublik.

Aus dem Inhalt: Internationale Strukturen in der informations- und kommunikationstechnischen Industrie - Industriepolitik für die Informations- und Kommunikationstechnik in Industrieländern - Struktur dieser Industrie in der BRD Industriepolitik in der BRD für die Informations- und Kommunikationstechnik Folgen für die Krisenbewältigung

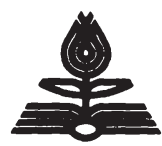

Verlag Peter Lang Frankfurt a.M. Bern • New York • Paris Auslieferung: Verlag Peter Lang AG, Jupiterstr. 15, CH-3000 Bern 15 Telefon (004131) 321122, Telex pela ch 912 651, Telefax (004131) 321131 